LASER ETCHED PMMA MICROFLUIDIC CHIP DESIGN AND MANUFACTURE WITH APPLICATIONS IN CAPILLARY ZONE ELECTROPHORESIS

\author{
A Thesis \\ Presented to the Faculty of \\ California Polytechnic State University, \\ San Luis Obispo \\ In Partial Fulfillment of the Requirements for the Degree \\ Master of Science in Biomedical Engineering \\ by \\ Evan A. Barbre
}


(C) 2011

Evan A. Barbre

ALL RIGHTS RESERVED

Page ii of $\mathbf{1 6 0}$ 
COMMITTEE MEMBERSHIP

TITLE:

LASER ETCHED PMMA MICROFLUIDIC CHIP DESIGN AND MANUFACTURE WITH APPLICATIONS IN

CAPILLARY ZONE ELECTROPHORESIS

AUTHOR:

Evan A. Barbre

DATE SUBMITTED:

FEBRUARY 2011

COMMITTEE CHAIR: $\quad$ David Clague, Ph.D.

Professor, Biomedical and General Engineering

California Polytechnic State University, San Luis Obispo

COMMITTEE MEMBER: Lily Laiho, Ph.D.

Professor, Biomedical and General Engineering

California Polytechnic State University, San Luis Obispo

COMMITTEE MEMBER: Richard Savage, Ph.D.

Professor, Materials Engineering

California Polytechnic State University, San Luis Obispo 


\title{
ABSTRACT \\ LASER ETCHED PMMA MICROFLUIDIC CHIP DESIGN AND MANUFACTURE WITH APPLICATIONS IN CAPILLARY ZONE ELECTROPHORESIS
}

\author{
Evan A. Barbre
}

This thesis encompasses a feasibility study of using low-cost materials to manufacture microfluidic chips that can perform the same functions as chips manufactured using traditional methods within an acceptable range of efficiency of chips created with more exotic methods and materials. The major parts of the project are the selection and characterization of the fabrication methods for creating the channels for fluid flow, the methods for sealing the channels to create a usable chip and the electrophoretic separations of carboxylated microspheres of different potentials. In this work we seek to answer the question if laser-etched PMMA microfluidic chips are comparable in functionality to microfluidic chips created with PDMS or glass. In the process of answering this question we will touch on FEA modeling, characterization of the manufacturing process and multiple prototype designs while keeping within the lowcost theme.

The purpose of capillary electrophoresis is to separate proteins based on their inherent electric charge. Capillary electrophoresis is a standard chip design used in the microfluidics world to prove a new fabrication method or chip material before branching out to other experiments because it is a fairly simple and robust design. Common problems associated with the manufacturing methods and materials were taken into account such as electroosmotic flow and chip sealing. CZE designs from literature were referenced to create a chip that would separate carboxylated microbeads with reasonable resolution. Wire electrodes were affixed to the chip to induce electric fields for the electrophoresis experiments. The goal of this thesis is to prove the manufacturing methods and attain results within $70 \%$ of literature standards.

Keywords: Capillary Electrophoresis, PMMA, Laser Processing, Low-Cost Microfluidics 


\section{AWKNOWLEDGMENTS}

I would like to thank my advisor Dr. David Clague for his support and guidance through the duration of the project. His steadfast and selfless support of student projects is an asset to the Biomedical Engineering program at Cal Poly.

I would like to thank my committee members Dr. Lily Laiho and Dr. Richard Savage for their assistance and support of my thesis defense

Lastly I would like to thank my father Greg, my mother Deborah and my sister Ariel for their incredible and steadfast support through the duration of my academic career. Without their motivation I would not have been able to complete this project. Thank you for always giving $110 \%$. 


\section{TABLE OF CONTENTS}

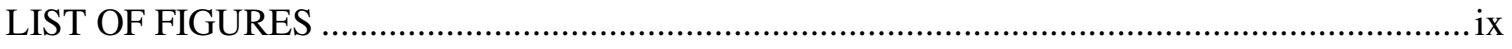

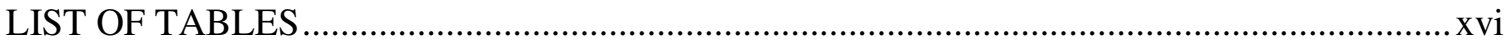

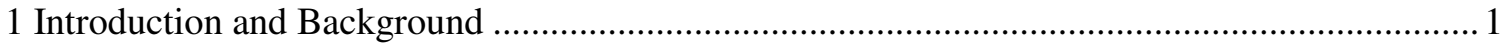

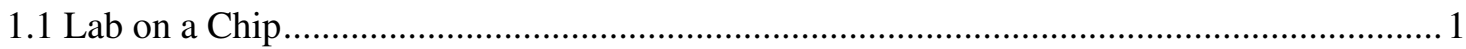

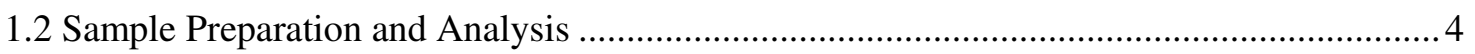

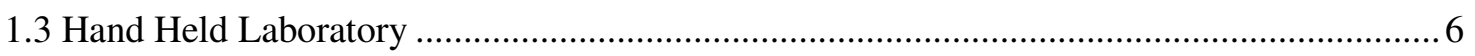

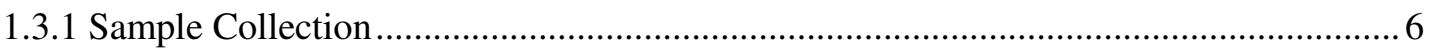

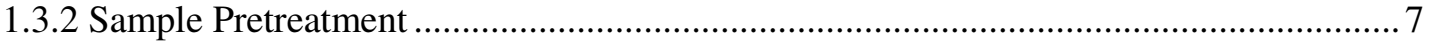

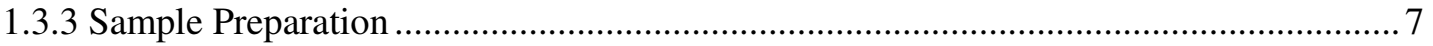

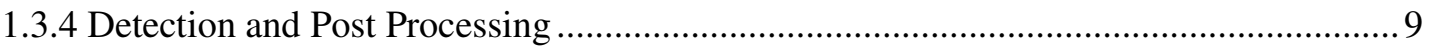

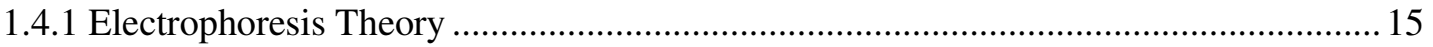

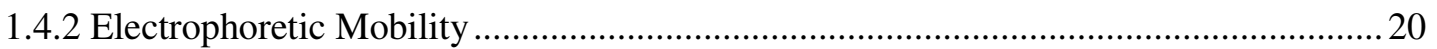

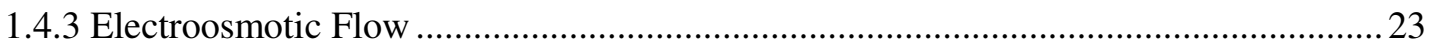

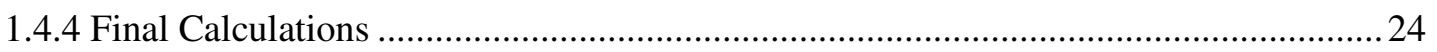

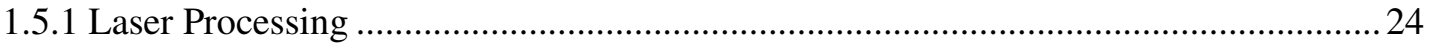

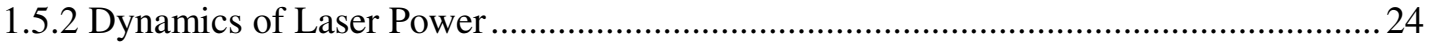

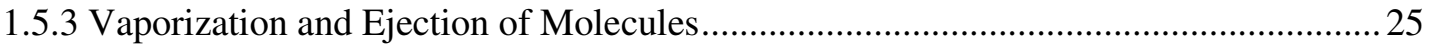

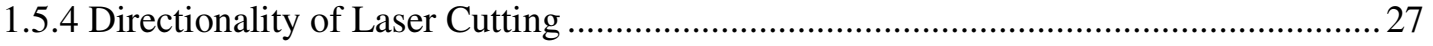

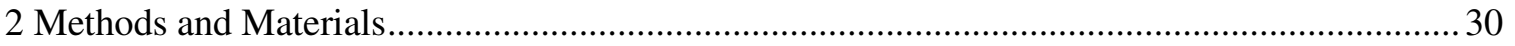

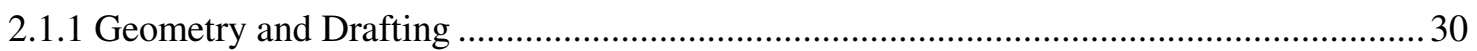

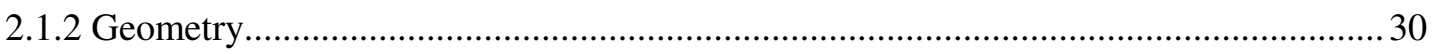

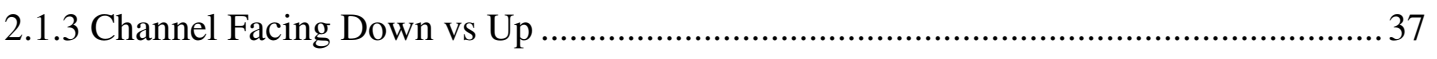

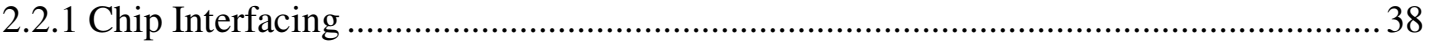

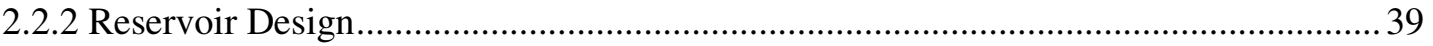

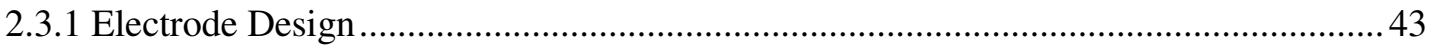

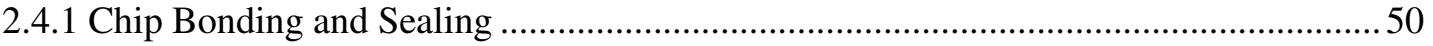

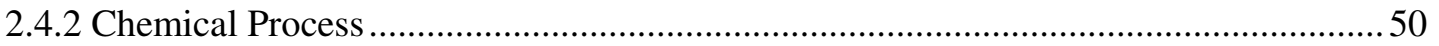

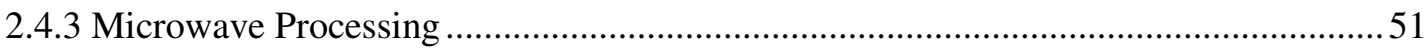

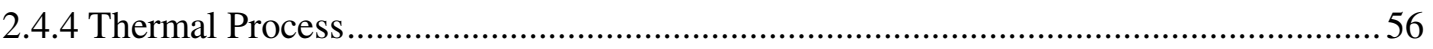

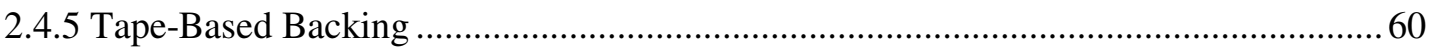




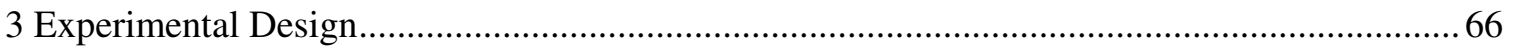

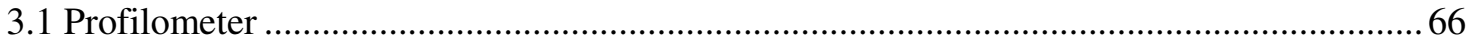

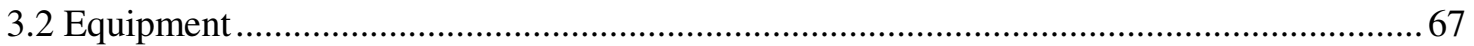

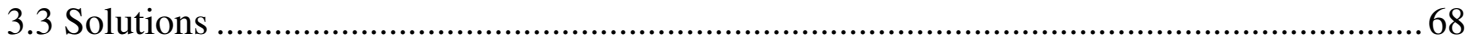

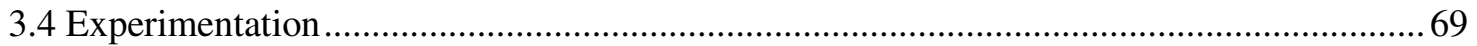

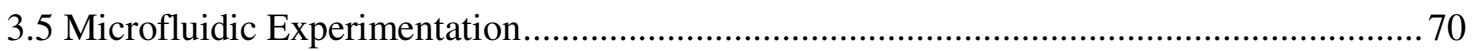

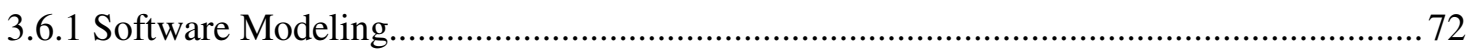

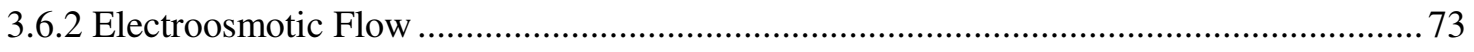

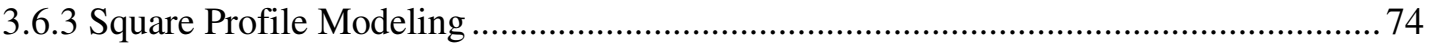

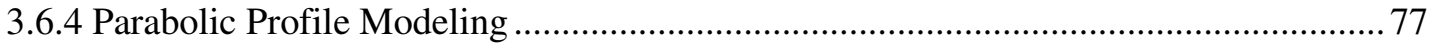

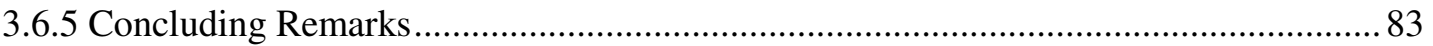

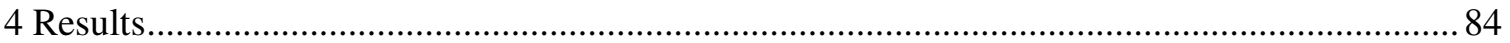

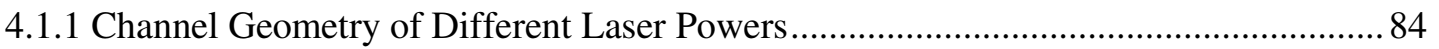

4.1.2 Comparison of Untreated and Heat-Treated Channel Geometry .................................. 89

4.1.3 Results for Multiple Laser Passes ............................................................................ 91

4.1.4 Comparison of Different Laser Powers Used in Raster Etching.................................95

4.1.5 Comparison of Channel Roughness versus Different Factors ..................................... 98

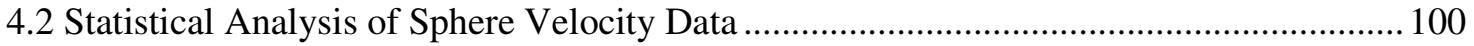

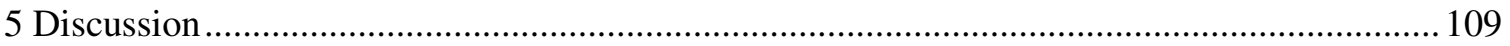

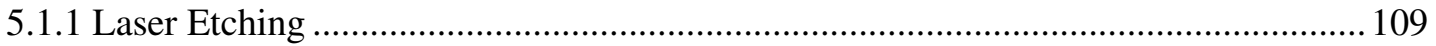

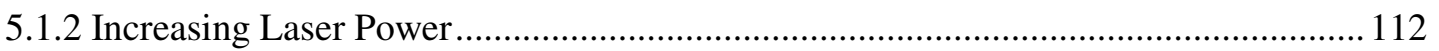

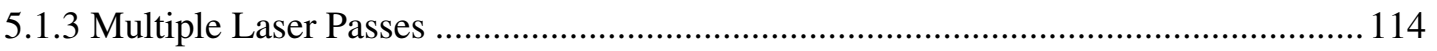

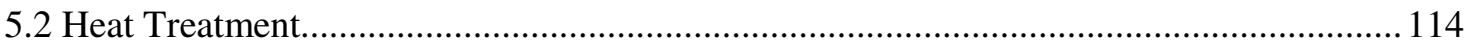

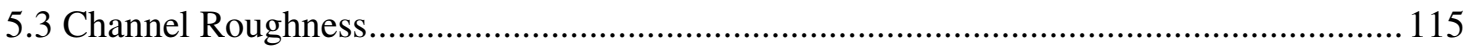

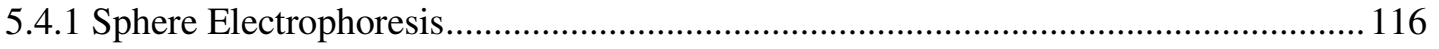

5.4.2 Comparison of Electrophoretic Mobility vs. Velocity …........................................... 118

5.4.3 Comparison of Particle Velocity vs. Data Collection Point......................................... 118

5.4.4 Comparison of Particle Velocity vs. Voltage ................................................................ 119

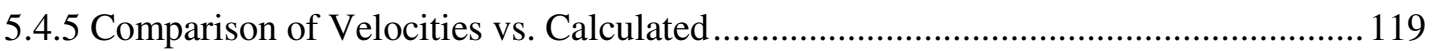

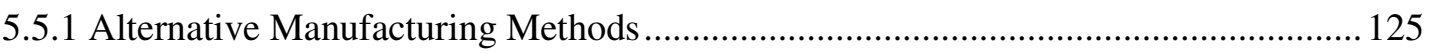

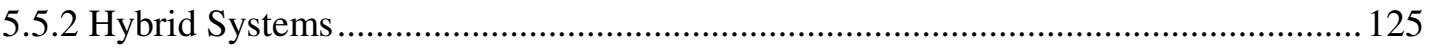




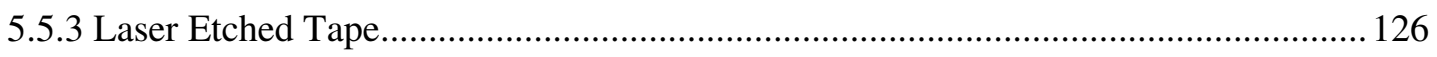

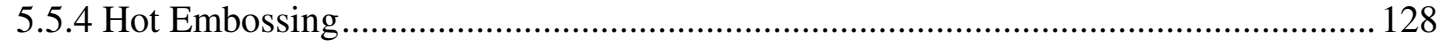

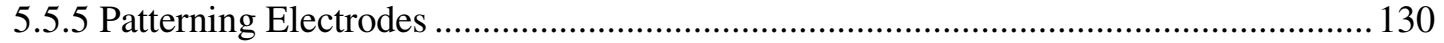

5.6 Other Microfluidics Chips Manufacturable With Laser Etched PMMA ........................... 131

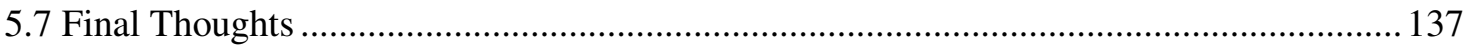

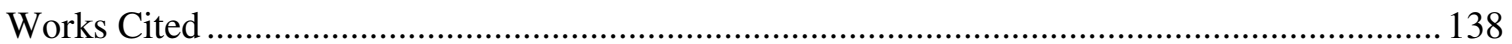




\section{LIST OF FIGURES}

Figure 1 - Abbot iStat blood analysis device ............................................................. 2

Figure 2 - Conceptual flowchart of a microfluidics diagnostic test.................................. 6

Figure 3 - Dimensioned CZE chip ........................................................................ 9

Figure 4 - Amino acid chain of Alanlyglutamylglycyllisine .......................................... 12

Figure 5 - Gel electrophoresis example ............................................................... 13

Figure 6 - Electropherogram result of a CZE experiment............................................... 14

Figure 7 - Illustration of method for detection in CZE using optical means ..................... 15

Figure 8 - Ionized forms of an amino acid as a function of $\mathrm{pH}^{27}$................................. 16

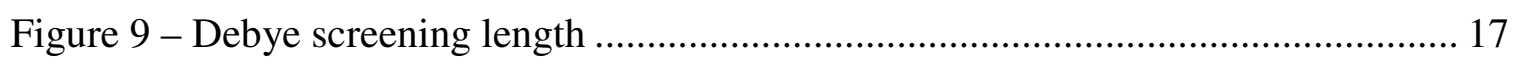

Figure 10 - Relaxation of Debye layer in a particle in motion ${ }^{27}$................................. 18

Figure 11 - Dynamics of laser ablation in PMMA ${ }^{31}$................................................. 25

Figure 12 - Profile of a laser-cut channel in PMMA using the 5\% power setting............ 26

Figure 13 - Graphical relationship between power, velocity and channel depth

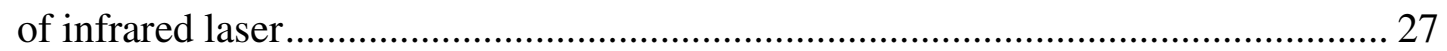

Figure 14 - Photograph of channels in PMMA creating a T-junction ............................. 28

Figure 15 - Directionality associated with sequences of laser cuts in PMMA ................ 28

Figure 16 - AutoCAD draft of electrophoresis chips...................................................... 29

Figure 17 - Standard CZE chip design ..................................................................... 30

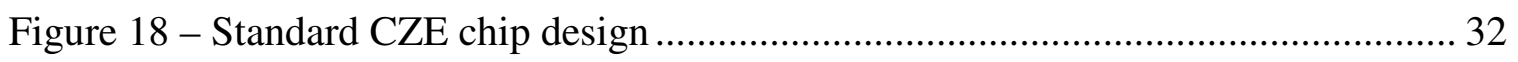

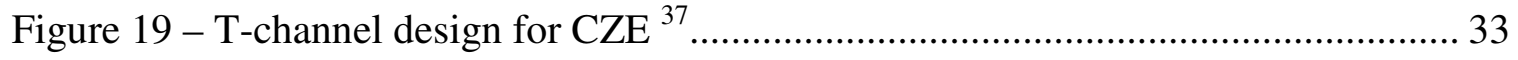

Figure 20- Step design for creating a sample "plug" ........................................................ 33

Figure 21 - Design for ‘step' CZE chip.......................................................................... 34

Figure 22 - Single buffer input design .................................................................. 34

Figure 23 - Optimization of serpentine channels using different geometries. ${ }^{42}$............... 35

Figure 24 - Serpentine separation channel CZE design. Scale bar is $1 \mathrm{~cm}^{53}$.................. 36

Figure 25 - 3-D PMMA chip design created to mix a sample with three different reagents and perform a separation .............................................................. 36 
Figure 26 - CZE chip geometry for multiple sample injections................................ 37

Figure 27 - Tolerance for tygon tubing insertion. ...................................................... 38

Figure 28 - Tubing connection created with injection syringe and 23 gauge needle....... 39

Figure 29 - CAD model for laser chip cutouts and through-holes for integration with tubing and syringe pumps and corresponding channel etches......................... 40

Figure 30 - Complete mockup of two-layered chip design ..................................... 40

Figure 31 - Hatch designs for raster cut reservoirs .................................................. 41

Figure $32-23$ Gauge through-holes for direct connection with hypodermic tubing....... 42

Figure 33 - Attenuation of laser power as a function of depth" ................................... 43

Figure 34 - 20-gauge electrode wire pierced through the tape backing

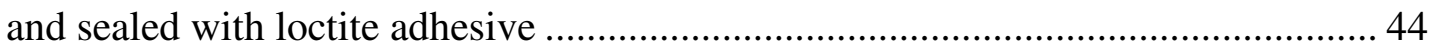

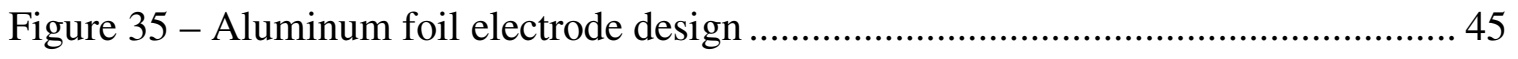

Figure 36 - Second electrode design involving aluminum foil. Foil is

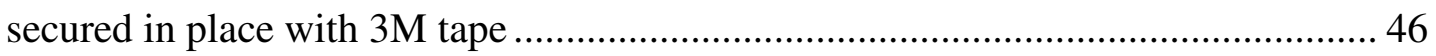

Figure 37 - Threaded electrode design with 40 gauge wire ........................................ 46

Figure 38 - 40 gauge wire threaded into tygon tubing and connected to 20 gauge

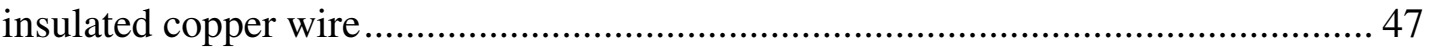

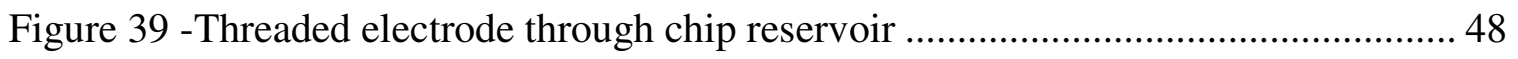

Figure 40 - Four electrode design with 20 gauge copper wire wrapped tightly around 23 gauge stainless steel hypodermic tubing .................................... 49

Figure 41 - Initial setup for microwave bonding .................................................... 51

Figure 42 - Experimental bonding setup used by Rahbar et al employing

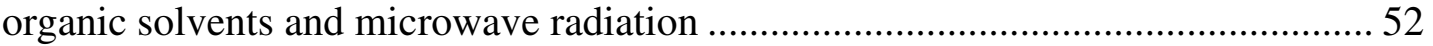

Figure 43 - Percentage of bonded area as a function of microwave time and solvent .... 53

Figure 44 - Initial trial of microwave-based chip bonding ....................................... 53

Figure 45 - Macroscopic view of chip damage during microwave bonding .................. 54

Figure 46 - Radiating patterns of damage in PMMA chip due to microwave

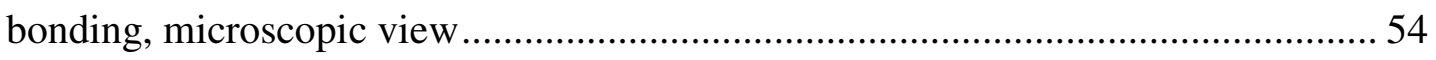

Figure 47 - Microscopic view of microwave bonded chip........................................ 55

Figure 48 - Leak testing of microwave bonded chip. ............................................... 55 


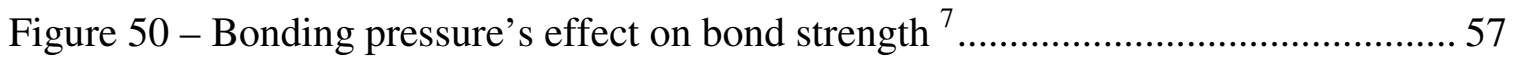

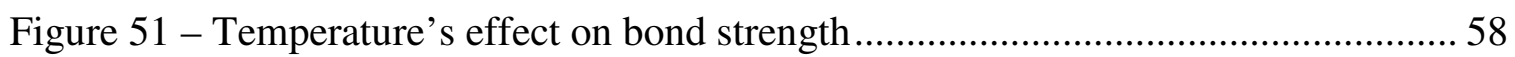

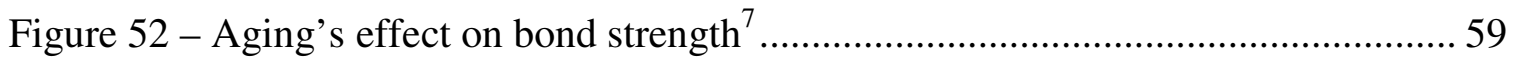

Figure 53 - Leak testing of thermally bonded chip and subsequent failure..................... 60

Figure 54 - Warped chip due to extended time at elevated temperature........................ 60

Figure 55 - Application of polypropylene packing tape onto back of chip.................... 62

Figure 56 - Visual artifacts of out-of-focus imperfections in polypropylene

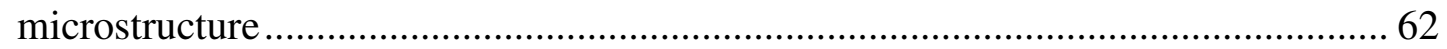

Figure 57 - Transparent tape used to seal the channels on a PMMA chip ...................... 63

Figure 58 - 10x view of 3M Transparent tape backing on PMMA microfluidic chip ..... 63

Figure 59-3M Transparent tape backing exposed to red light wavelengths and covered with the SVM chip cover that protects the chip from room lighting at $10 \mathrm{X}$ magnification 64

Figure 60- Microsphere shown beneath fluorescent tape.

Figure 61-Computer model of a profilometer tip. Notice the laser beam reflecting off the top of the cantilever ${ }^{48}$ 66

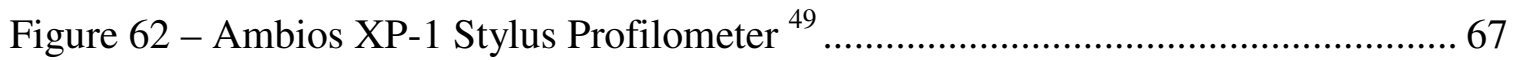

Figure 63 - Labeled experimental setup. 1......................................................... 71

Figure 64 - Setup in use with SVM LEDs. Experimental foil electrodes are in use in this picture (L). Close-up of final electrode design in use (R) ................. 72

Figure 65 - Electroosmotic flow profile of a parabolic profile channelt......................... 73

Figure 66 - Electroosmotic model of a square profile channel 120 microns by 120

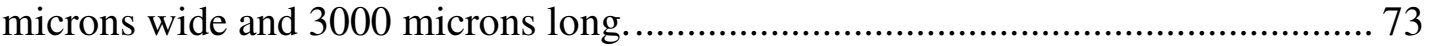

Figure 67 - Slices showing fluid flow along channel of parabolic profile channel ........ 74

Figure 68 -3-dimensional velocity field arrows of square profile channel

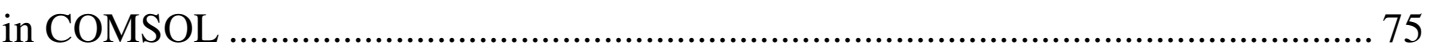

Figure 69 - Top-down view of velocity profile of square channel from middle of the channel and 70 microns into the channel ............................................... 75

Figure 70 - Graphical velocity profile of flow through the center of the

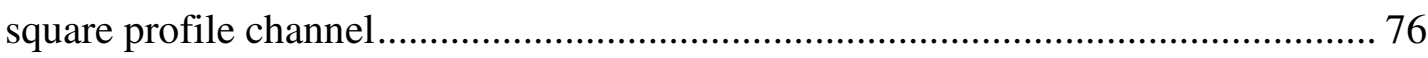


Figure 71 - Velocity along centerline of square profile channel................................ 77

Figure 72 - Flow velocity slices of a parabolic channel............................................ 78

Figure 73 - 3-dimensional velocity field arrows of parabolic profile channel................ 78

Figure 74 - Velocity field from top to bottom of parabolic channel .............................. 79

Figure 75 -Velocity graph across the parabolic profile channel 20 microns

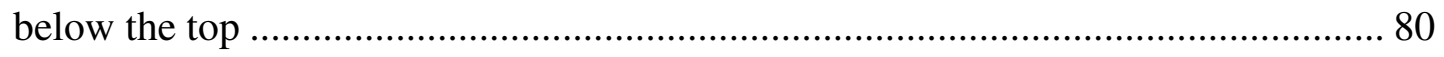

Figure 76 - Velocity graph across the flow in a parabolic profile channel

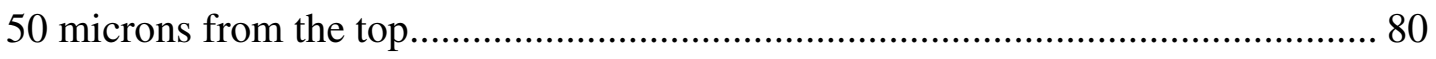

Figure 77 - Top-down view of parabolic profile with (L) halfway down the

depth of the channel and (R) $1 / 3$ of the way into the depth of the channel.............. 81

Figure 78 - Side view of velocity graph with middle of the channel and

$1 / 3$ of the way into the channel from the side..................................................... 82

Figure 79 - Velocity field down center of parabolic profile channel in area

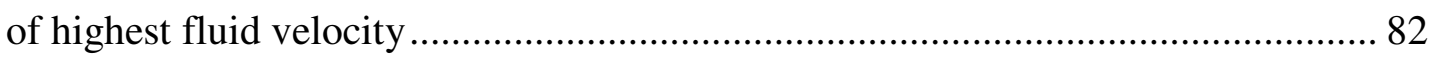

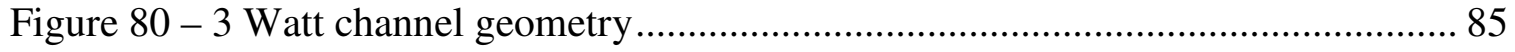

Figure 81 - Channel geometry of a single pass with a 4 watt laser............................... 85

Figure 82 - Channel geometry of a single pass with a 5 watt laser ............................... 85

Figure 83 - Channel geometry of a single pass with a 6 watt laser............................... 86

Figure 84 - Comparison graph of channel geometries etched with

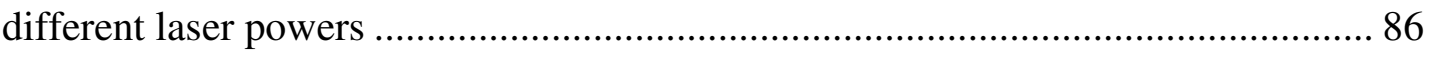

Figure 85 - Graph comparing depth of channel versus laser power.............................. 87

Figure 86 - Graph comparing channel width to laser power..................................... 87

Figure 87 - Graph comparing ejected material versus laser power .............................. 88

Figure 88 - Graph comparing lip height versus laser power with linear

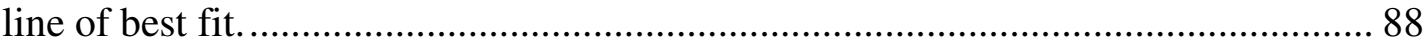

Figure 89 - Comparison of untreated and heat-treated channel geometry

for a channel etched with a 10.6 micron wavelength $\mathrm{CO}_{2}$ laser at 4 watts .............. 89

Figure 90 - Comparison of untreated and heat-treated channel geometry for

a channel etched 10.6 micron wavelength $\mathrm{CO}_{2}$ laser at 5 watts. ........................... 89

Figure 91 - Comparison of untreated and heat-treated channel geometry

for a channel etched with a 10.6 micron wavelength $\mathrm{CO}_{2}$ laser at 6 watts ...............90 
Figure 92 - Comparison of maximum depth of channels versus laser power with logarithmic lines of best fit drawn along with respective $\mathrm{R}^{2}$ values 90

Figure 93 - Graph of ejected material from the channel versus laser power with a

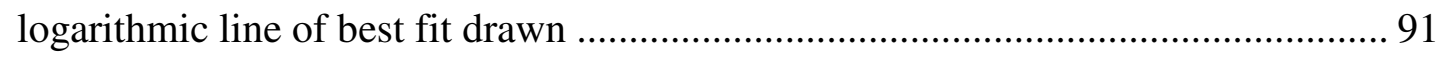

Figure 94 - Channel geometry of a channel after two passes with a 3 watt laser ........... 91

Figure 95 - Channel geometry of a channel after three passes with a 3 watt laser ......... 92

Figure 96 - Overlaid comparison of the same laser power passed multiple

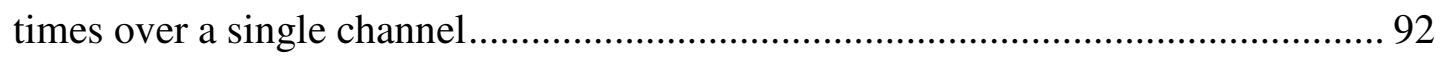

Figure 97 - Graph comparing the width of channels after multiple passes with a

logarithmic line of best fit, its equation and $\mathrm{R}^{2}$ value ....................................... 93

Figure 98 - Height of redeposited material at edges of the channel

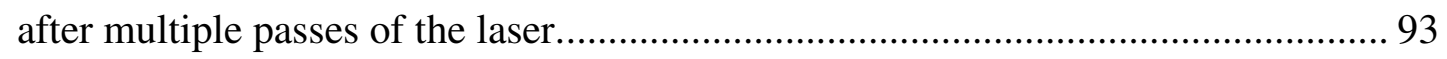

Figure 99 - Maximum depth of channel versus number of laser passes 3 watt $\mathrm{CO}_{2}$...... 94

Figure 100 - Material ejected from the channel versus number of laser

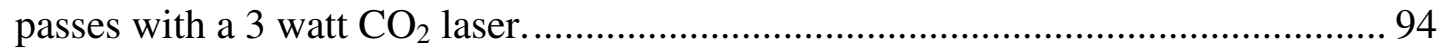

Figure 101 - Geometry of a raster cut created with a 2 watt $\mathrm{CO}_{2}$ laser ........................ 95

Figure 102 - Geometry of a raster cut created with a 3 watt $\mathrm{CO}_{2}$ laser ........................ 95

Figure 103 - Geometry of a raster cut created with a 4 watt $\mathrm{CO}_{2}$ laser ......................... 96

Figure 104 - Geometry of a raster cut created with a 5 watt $\mathrm{CO}_{2}$ laser ........................ 96

Figure 105 - Overlaid comparison of raster etching powers of $\mathrm{a} \mathrm{CO}_{2}$ laser .................. 97

Figure 106 - Scatterplot of amount of material ejected from rastering process

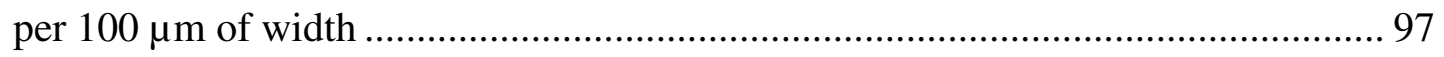

Figure 107 - Roughness along channels with multiple passes of a 3 watt laser. ............ 98

Figure 108 - Comparison of channel roughness versus 4 and 5 watt

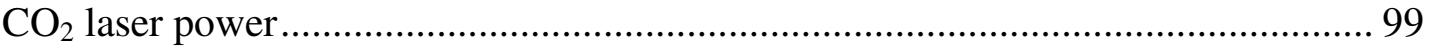

Figure 109 - Comparison of effects of heat treatment versus no treatment on roughness of channels created with the same 4 watt $\mathrm{CO}_{2}$ laser power................ 99

Figure 110 - Overlay graph of all methods of fabrication used for laser etched channels

Figure 111 - Scatterplot of microsphere velocity versus voltage of 1 micron particles with polynomial line of best fit and 95\% confidence interval...... 106 
Figure 112 - Interval plot of 1 micron particle velocity with a 95\% confidence level used. 106

Figure 113 - Scatterplot of velocity versus voltage of 5 micron particles with polynomial line of best fit and $95 \%$ confidence interval.

Figure 114 - Interval plot of 5 micron particle velocity with a 95\% confidence level.

Figure 115 - Scatterplot of velocity versus voltage of 10 micron particles with polynomial line of best fit and $95 \%$ confidence interval. 108

Figure 116 - Interval plot of 10 micron particle velocity with a $95 \%$ confidence level. 108

Figure 117 - Graph relating laser translational speed and power with depth indicated by color

Figure 118 - Depth vs. Power graph of a CO2 laser travelling at $381 \mathrm{~mm} / \mathrm{s}$

Figure 119 - Three examples of evidence of "fat finger syndrome" inherent to probe-based microscopy

Figure 120 - Laser-etched microchannel before and after thermal annealing at $170{ }^{\circ} \mathrm{C}$ for $30 \mathrm{~min}$.

Figure 121 - Percentage deviation of experimental electrophoretic mobilities from theoretical values

Figure 122 - Graph of correction factor versus voltage for 5 and 10 microsphere data

Figure 123 - Hot embossed PMMA microfluidic device with 50 micron

PDMS layer at the interface. 126

Figure 124 - Tape-based microfluidics device manufacturing process

Figure 125 - SEM comparison of surface roughness between direct-etched

PMMA and PDMS poured into a laser-etched tape mold 128

Figure 126 - Fabrication method for wire embossing compared to embossing method with silicon mold

Figure 127 - Microarchitecture created in PMMA by hot embossing ${ }^{68}$ 130

Figure 128 - Embedding solder wire into microfluidic chip as an electrode ${ }^{71}$ 131

Figure 129 - AutoCAD drawing of a proposed H-Filter for fabrication with a laser cutter 


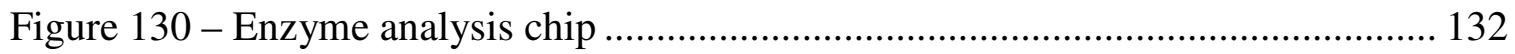

Figure 131 - More complicated enzyme assay chip ............................................... 133

Figure 132 - Design of microfluidic chip adapted from David Pescetti’s

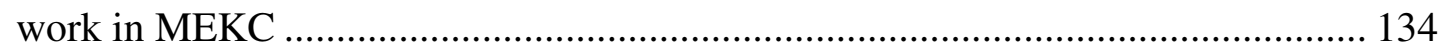

Figure 133 - AutoCAD design for passive 3-D serpentine mixing chip ....................... 134

Figure 134 - Successively increasing levels of mixing of water and alcohol

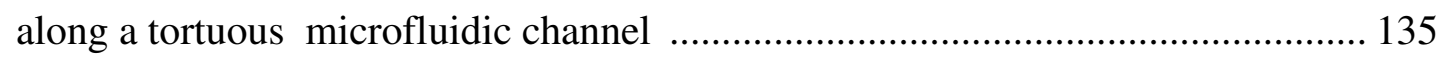

Figure 135 - Series mixing microfluidic device with rough channel floor to facilitate

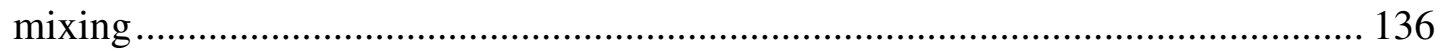




\section{LIST OF TABLES}

Table 1 - Carboxyl group surface density and net potential for microspheres ................ 22

Table 2 - Electrophoretic mobility of microspheres .................................................... 24

Table 3 - Chart listing the results of the T-test comparison of 1 and 5 micron particles at different voltages and locations.

Table 4 - Chart listing the results of the T-test comparison of 5 and 10 micron particles at different voltages and locations.

Table 5 - Chart listing the results of the T-test comparison of 1 and 10 micron particles at different voltages and locations.

Table 6 - Chart listing the results of the T-test comparison of 1 micron particles the same voltage at different locations

Table 7 - Chart listing the results of the T-test comparison of 5 micron particles the same voltage at different locations

Table 8 - Chart listing the results of the T-test comparison of 10 micron particles the same voltage at different locations

Table 9 - Chart comparing 1 micron particle movement at the same location while under the influence of different voltages.

Table 10 - Chart comparing 5 micron particle movement at the same location while under the influence of different voltages

Table 11 - Chart comparing 10 micron particle movement at the same location while under the influence of different voltages.

Table 12 - Expected velocities in microns/second of microspheres

Table 13 - Percent error difference of experimental electrophoretic velocities from theoretical

Table 14 - Comparison of theoretical and experimental values for sphere velocity data

Table 15 - Correction factors for sphere velocities based on size and voltage

Table 16 - Average percent error from theoretical values for corrected data compared to original error 


\section{Introduction and Background}

\subsection{Lab on a Chip}

Lab-on-a-chip technology (LOC) has existed since the 1990's as a way to perform miniaturized versions of large-scale experimentation. LOC applications have the advantage of being easily transportable and readily automated - well suited for developing countries in particular. There is a great need in developing countries for diagnostic technologies, as timely and accurate diagnostics have been identified as a

means to greatly reduce treatment costs ${ }^{1}$. Many of the disease-associated deaths in developed countries, like heart disease and cancer are not communicable and are not considered as great a concern for each community. In contrast, transmissible diseases are a cause of much greater concern in poverty-stricken developing countries and point-ofcare (POC) diagnostics can prevent an outbreak by justifying the quarantine of an individual. Identifying and halting the spread of a communicable disease is ultimately much more beneficial in this context. Furthermore, the limited amount of hospitals and medical personnel in developing countries also necessitates the development of diagnostic tools that can be used by untrained or casually trained operators. Enabling an accurate diagnosis of a patient in the field will allow medical personnel to be in a state of readiness for the particular patient. The Bill and Melinda Gates Foundation noted that two of the 14 priorities for creating a healthier world were in the areas of diagnosis and measurement. 
Developing countries require diagnostic technologies that require little or no electricity, operators with little or no training and robust technologies that can handle extreme environmental conditions as well as rough handling during transportation and use. On top of these constraints, the device must be low cost compared to other methods of testing. Many microfluidic chips can be automated with syringe pumps that can be set to dispense fluids at a precise rate that can also be run on batteries. One such device is called the i-Stat from Abbot Laboratories. ${ }^{2}$

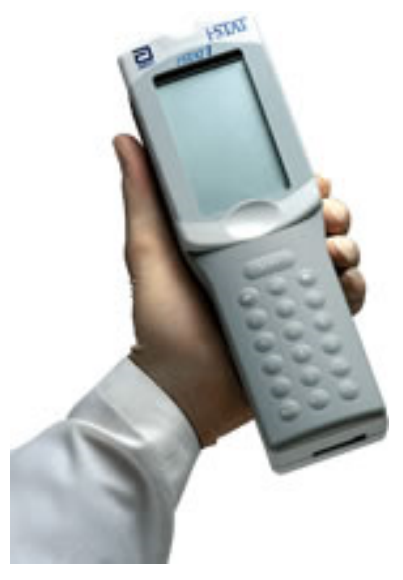

Figure 1 - Abbot iStat blood analysis device

iStat point-of-care analysis device. The iStat device is capable of measuring sodium, potassium, chloride, ionized calcium, blood gas values ( $\mathrm{pH}$, pO2, $\mathrm{PCO} 2, \mathrm{HCO} 3, \mathrm{TCO} 2, \mathrm{BE}, \mathrm{SO} 2$ ), as well as anion gap, hemoglobin/hematocrit, urea, and glucose ${ }^{2}$

Several microfluidics chips, such as the electrophoresis chip described in this thesis, require source of power to. Additionally, many bench-scale assays require a fair amount of materials, reagents and buffers for a single test. In contrast, microfluidics chips, as the name suggests, use fluid amounts on the scale of microliters and require far less power. 
This not only reduces the amount of reagents required to perform a desired test but it also significantly reduces required test volumes e.g. blood or saliva from the test subject.

Many important developments have been made in the field of microfluidics that have allowed previously unconsidered experimentation possible, including DNA assays, single-cell capture, cell separation based on size and type, micellar separations, cell culture/incubation, sample preparation and even spectroscopy ${ }^{3,4}$. However, few commercial products have come about based on the research being performed in this field from the difficulty in manufacturing a robust product.

Much of microscale manufacturing is performed using a polymer called polydimethysiloxane, or PDMS, using processes such as photolithography and replica molding. ${ }^{5}$ The advantages of using PDMS include its ability to fill molds with very precise microarchitecture, optical clarity, and its ability to easily bond to glass slides with use of a plasma gun. Using these processes allows extremely fine detail, down to about 2 microns using the mold-pouring end process. ${ }^{6}$ Extensive research efforts with PDMS have yielded functional structures such as valves, lenses, passive mixing structures and functionalized group attachment ${ }^{7,8}$ However, PDMS cannot be exposed to high temperatures for long periods of time and has a relatively short shelf-life. PDMS will tear when subjected to high amounts of stress as it is much like a weak rubber at room temperatures. ${ }^{4}$ As a consequence, PDMS chips can only be reliably handled by trained personnel. Furthermore, the lack of mechanical stiffness can lead to sagging which may block large channels or chambers. ${ }^{4}$ The process from raw material and mold to finished 
chip requires expensive and caustic chemicals as well as expensive and sensitive equipment that cannot be transported easily. ${ }^{4}$ As such, PMDS is best suited for prototyping microfluidic designs. For point-of-care diagnostics far away from capable facilities we must look elsewhere for the proper materials and manufacturing processes.

Polymethylmethacrylate, or PMMA, has been proposed as an alternative material for manufacturing microfluidic devices. This material has the advantage of being mechanically stiffer, more stable, more hydrophilic, and less expensive than PDMS. 9,10 It has the advantage of being fairly optically clear over other polymers and is very readily available commercially under the popular monikers plexiglass and Lucite. PMMA microfluidics chips also can be manufactured using lithographic processes to achieve down to $6 \mathrm{~nm}$ size features. ${ }^{11}$ Microfluidics chips manufactured from PMMA can also be created by other methods such as UV patterning, laser photoablation, hot embossing, $\mathrm{x}$ -

ray lithography, ion beam etching and plasma etching. ${ }^{12}$ Based on the resources available at Cal Poly, the works presented in this thesis will focus on photoablation.

\subsection{Sample Preparation and Analysis}

A raw sample, be it blood or otherwise, must be processed before it can be properly tested. A test looking for a certain protein in the blood will most likely not work as it may clog the microchannels in an LOC or overrun a small test site with too much material and render it useless. Many biochemical tests require fairly exact sample parameters such as $\mathrm{pH}$, viscosity and target concentration and bringing a sample from patient to a testable state requires dilution, buffering and filtering. In some cases concentration or amplification is required to enable detection. These representative 
pretreatment steps are aimed at isolating and purifying target markers biomolecules to enable accurate and early detection. The details of the pretreatment and sample preparation steps will not be covered in detail in this thesis; rather, this thesis is focused on characterization of ablation as a means of manufacturing desired components.

Sample analysis refers to the actual portion of the experimentation where an actual analysis is performed. There are a host of different tests that can be performed in LOC capacities. Some examples of tests relevant to developing nations include: blood enzyme analysis; measurement of blood gases, electrolytes, lipids, thyroid indicators and drugs, flow cytometry, tests for diseases such as HIV/AIDS, tuberculosis and rotavirus, and separation of proteins based on size and charge for analysis. ${ }^{13,14,15}$ This last approach, separation based on size and charge, is known as electrophoresis. As part of the work document in this thesis, PMMA capillary electrophoresis chips were manufactured using laser ablation and characterized. 


\subsection{Hand Held Laboratory}

While microfluidic devices produce results when operated by an experienced technician, they are nearly unusable without training. This technology will not acquire relevancy and widespread use unless device platforms become user-friendly. With this perspective in mind, this project is part of a larger-scope project to create a handheld laboratory that will take a raw biological sample - be it blood, sputum, semen, sweat or otherwise - and process target analytes are detected. Below is a figure describing the areas the larger-scope project covers.

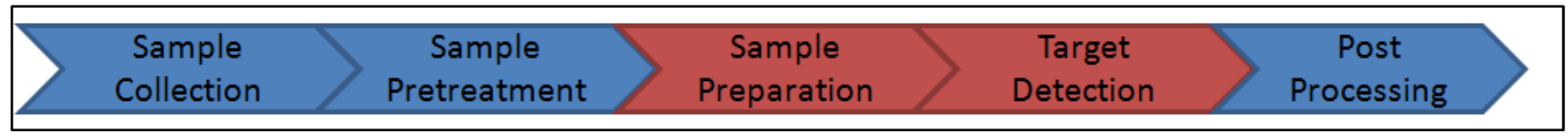

Figure 2 - Conceptual flowchart of a microfluidics diagnostic test Scope of microfluidics projects. The red arrows correspond to the possible uses for electrophoresis chips

\subsubsection{Sample Collection}

Collection of biological samples can be a difficult process when performing biological analysis, as contamination of the sample can yield wildly varying results from even the same subject. Blood plasma and blood serum are two commonly used biological samples for analysis. Blood plasma refers to the yellowish liquid blood component that contains ions, proteins, glucose, hormones, fibrinogens and platelets suspended in water. Blood serum refers to blood plasma without the proteins involved in clotting. ${ }^{16}$ Blood or blood serum is one of the most commonly used biological samples because it gives one of the best representations of the current health state of that subject, whereas sputum, urine or stool samples can yield various results dependent on recent diet or activity. Acquiring a blood sample is also much more difficult to obtain outside of a laboratory environment and 
requires sterile needles which can prove to be costly. Furthermore, blood requires the use of anticoagulants in microfluidics environment to ensure device functionality; however, some assays may require compensation for the anticoagulant prior to detection. An example of this is the compensation in the graphs produced by an optical coherence tomography device when measuring hematocrit levels in blood based on the level of red blood cell aggreagation. $^{17}$

\subsubsection{Sample Pretreatment}

After collection, the process of pretreatment conditions the sample for downstream separation steps by adding specific amounts of molecules and ions to reach certain fluid parameters. The important parameters particular to this thesis are target concentration, solution conductivity and surfactant concentration. Conductivity is of critical importance for electrophoresis. In particular, DI water has a resistance of $18 \mathrm{M} \Omega$ and will not conduct electricity. When using microspheres, the surfactant concentration a must be held within certain bounds as well to prevent clumping. The microspheres used in experimentation will likely flocculate within the solution without a surfactant. Other modifications during sample pretreatment may include the addition of enzymes. An example of this would be the pretreatment of cerebro-spinal fluid before storage with methanol. Zhang et al found that pretreating CSF before storage at $-80^{\circ} \mathrm{C}$ would keep concentrations of amino acids at more stable levels than without. ${ }^{18}$

\subsubsection{Sample Preparation}

As described previously, the sample will need to be within certain fluid parameters to enable a successful phoresis and detection. The sample will need to be at a certain $\mathrm{pH}$ to reduce denaturing of sensitive proteins or specific antibodies for binding. In the case of this thesis, the $\mathrm{pH}$ had to be at a certain level to ensure a charged species. 
Viscosity of sample fluids is of great importance. Whole blood is highly viscous and usually must be diluted with a buffer before experimentation. Microfluidic chips are very small-scale devices and pumping highly viscous fluids through small channels can create intensely high local pressures that cause problems within the chip such as leaks, breaks or tears in the case of PDMS chips.

Target concentration also becomes an important issue when testing biological samples. In many cases, qualitative tests for cells, nucleic acids or proteins require a certain threshold concentration before they elicit a result. A common example is polymerase chain reaction or PCR. In PCR, DNA is replicated until a detection threshold is achieved. In other cases, such as an ELISA HIV tests, the sample must be diluted up to 400:1 concentration for a proper testing environment. Pretreatment may refer to many different processes, including buffer addition, particulate suspension, component isolation or dervatization in preparation for detection processes. ${ }^{19}$

One method for sample preparation and detection is Capillary Zone Electrophoresis, or CZE. Electrophoresis can be utilized during sample preparation for separation of certain charged species from others in the sample. Two different fluorescent tagged proteins with different electrophoretic mobilities can be separated and analyzed for concentration using methods found in the electrophoresis section of the introduction. Additionally, using a twodimensional chip design with a perpendicular cross-channel at the end of the separation channel a chip can temporarily create a voltage potential across the cross-channel to separate certain molecules from the rest of the sample. 


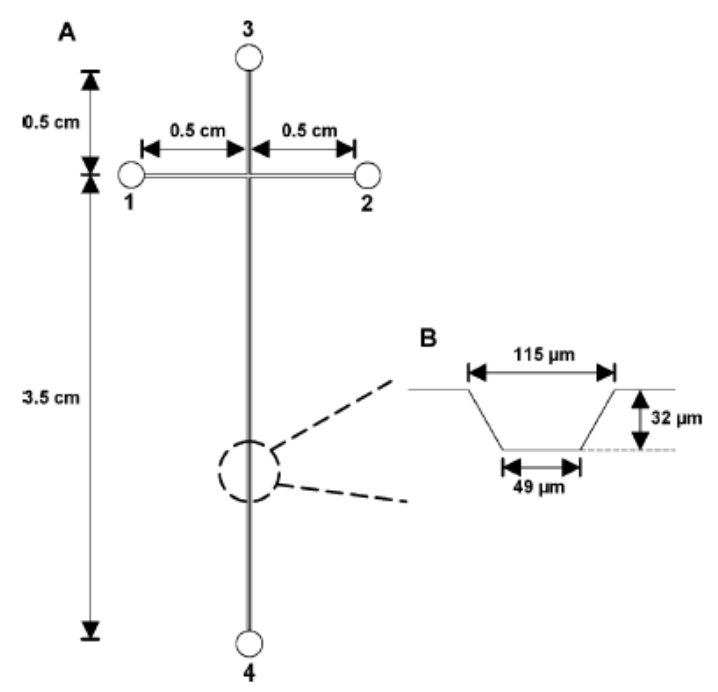

Figure 3 - Dimensioned CZE chip

Starting from the top and moving clockwise, the circular ports are for buffer, sample injection, buffer and waste. ${ }^{20}$

\subsubsection{Detection and Post Processing}

The previous steps are required, but in the end detection and post processing yields the relevant data for clinical decision makers. There are many approaches for target detection. For example, immunodetection is a common method used that employs the use of very specialized glycoproteins called antibodies to bind to specific antigens either in blood, on a protein or on the surface of a cell in a process called induced fit. ${ }^{19}$

Other methods are also in use, including fluorescence of molecules. By using optical filters, the sample can be exposed to small ranges of wavelengths of light that correspond to specific absorption frequencies of fluorescent molecules. Using the same filters, a small band of emission spectra can also be analyzed either visually or by software.

Commonly, capillary electrophoresis experimentation will yield electropherograms like the one shown in the electrophoresis section. Electrophoresis can be used for detection by 
comparing the electrophoretic velocity of a protein band in a sample against a known species that has been added during the sample preparation stage, or by calculating the electrophoretic mobility of a sample based on the knowns of the properties of the fluid, electric charge and channel length.

\subsubsection{Electrophoresis in Diagnostics and Detection}

Electrophoresis is commonly used in diagnostics when assessing relative amounts of proteins or peptides. Common methods of analysis include DNA sequencing to determine genetic disease, bacterial infections, viral DNA detection, and analysis of genetic

polymorphism. ${ }^{3}$ These are attractive uses of the platform when taken alongside the speed of detection CZE offers.

\subsubsection{Project Goals}


The purpose of this project is to test the viability of PMMA as a microfluidic device material. The PMMA will be laser etched to create sub-millimeter channels for flow of fluids and electrophoretic separation of charged microspheres. It is important that this project demonstrates how to go about creating a microfluidic platform using low-cost materials and laser cutting equipment and suggests areas that future students can delve into.

1) Demonstrate knowledge of electrophoresis 2) Determine proper laser settings for creating a microfluidic chip using a laser cutter 3) Design and manufacture a microfluidic platform using low-cost materials 4) Create an electrophoretic experiment with charged microspheres to demonstrate functionality of microfluidic chip 5) Compare results of microfluidic experimentation against COMSOL and Peak Master models as well as theoretical calculations 6) Create AutoCAD models of other microfluidic experiments as suggestions for future graduate students and provide them with knowledge of laser cutter usage.

\subsubsection{Electrophoresis}

Electrophoresis describes the motion of charged species in solution when subject to an electric field. One class of charged species that is commonly manipulated by electrophoresis is proteins. Every protein is composed of a chain of amino acids, each with their own electric charge. In its folded state, a protein will have a certain net charge that it can be classified with, usually dependent on the $\mathrm{pH}$ of the solution it is in. ${ }^{21}$ Proteins will display a unique charge, which allows for separation and identification. 


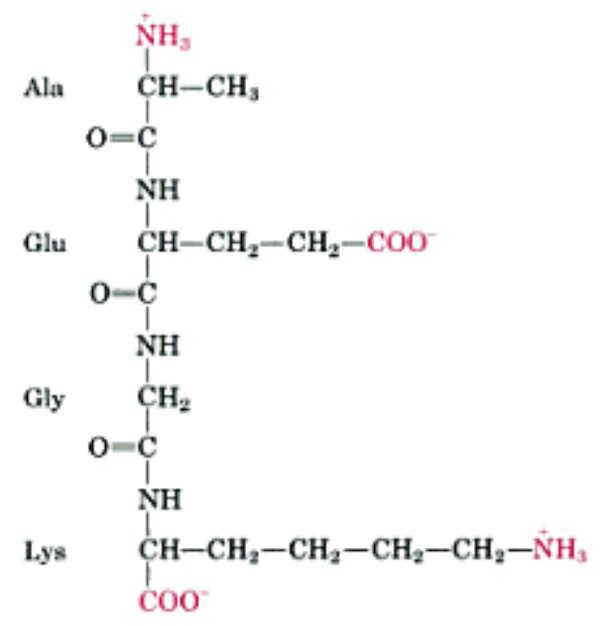

Figure 4 - Amino acid chain of Alanlyglutamylglycyllisine

Shown in red are the groups that are ionized at $\mathrm{pH} 7^{21}$

In addition to categorizing proteins based on charge, they may also be classified by their size. $^{21}$ Electrophoresis of proteins has been around since Dr. Tiselus' thesis “The Moving Boundary Method to Study The Electrophoresis of Proteins" in 1930 where he was able to separate a- b- g-globulin and detect them by observing the refractivity of their boundaries. 22

During an electrophoretic process, proteins are separated by their electric charge. One of the most common forms of electrophoresis is gel electrophoresis. In gel electrophoresis, protein laden samples are loaded into a gel slab in one end and a voltage potential is applied across the length of the gel. The gel forms a sieving media and separates by size and charge as the proteins must navigate the gel matrix while under the influence of the electric current. After a certain amount of time has passed (determined by gel slab length, voltage and gel slab material), the slab is exposed to a visualizing medium, usually UV light, which exposes how 
far the proteins have travelled. Shown below is an example of a gel electrophoresis experiment. The proteins are loaded into small troughs at one end of the slab and roughly keep their shape during the course of travel. ${ }^{21}$

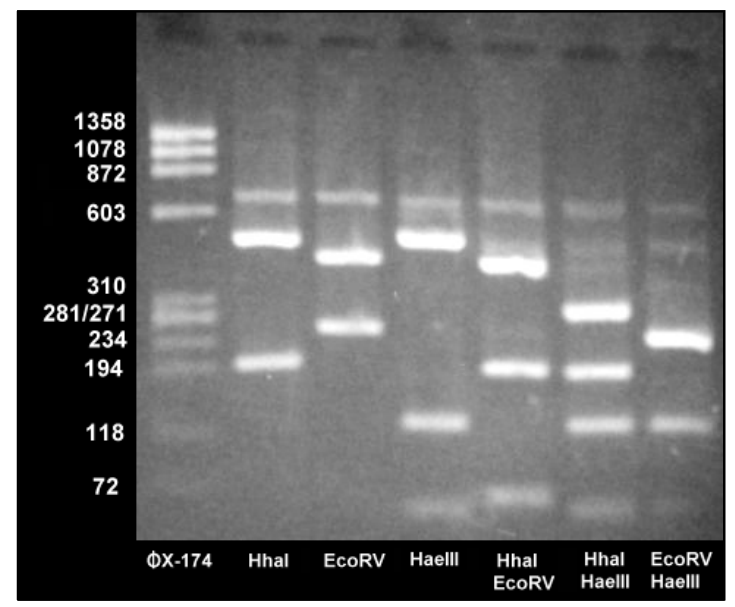

Figure 5 - Gel electrophoresis example

Numbers on the left of the picture indicate protein size in bp, or base pairs for each of the fractions. The injection troughs can be seen at the top of the picture as dark ovals. ${ }^{23}$

\subsubsection{Capillary Electrophoresis}

In capillary zone electrophoresis, a small channel is used to separate the proteins and initially came about when Dr. Hjerten found that electrophoresis in a tube reduced the thermal effects of heating by electric charge. Because it operates in a channel with a stationary detector, there is no stationary phase in capillary electrophoresis as there is in gel electrophoresis. The name comes from the distinct 'zones' that are created when the electric charge is applied, as in the picture for gel electrophoresis. ${ }^{22}$

Only one experiment may be run at a time but capillary electrophoresis has the distinct advantage of being able to be used multiple times. There are several types of experiments within the blanket of capillary electrophoresis including gel and free solution. Most CZE 
experiments use the free solution as this method is easier to prepare than loading a tube with a gel. Gel electrophoresis experiments usually take on the range from 15-30 minutes, whereas CZE experiments usually take on the range of 2-10 minutes although some researchers are seeing progress in CZE separations and have been able to reduce the time to less than two minutes. ${ }^{24}$ Instead of analyzing a slab, capillary electrophoresis requires some sort of image analysis software that will 'watch' a window at the end of the channel and report the amount of protein 'seen'.

There are several different types of detection with CZE, including optical absorbance detection, thermo-optical absorbance sensing, fluorescence detection, electrochemical detection, radioactivity detection, mass spectral detection, nuclear magnetic resonance detection and laser-induced fluorescence detection. All of these methods have some sort of detection method and in the current era of electrophoresis output to a computer with processing software. Below is a graph of what capillary electrophoresis experiments produce. $^{22}$

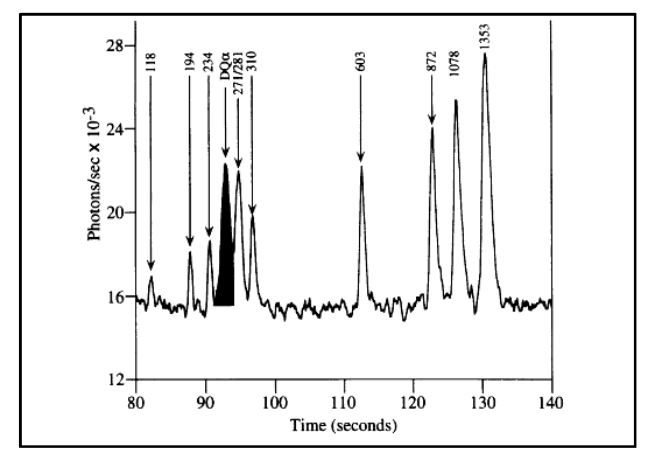

Figure 6 - Electropherogram result of a CZE experiment

High-speed sizing of PCR-amplified DNA fragments on a capillary array electrophoresis chip Vertical peaks are related to relative amounts of DNA fragments versus time on the horizontal axis ${ }^{25}$ 
For optical detection methods two methods are commonly used. The older standard has been the combination of a light source and a photodetector as shown in the following diagram.

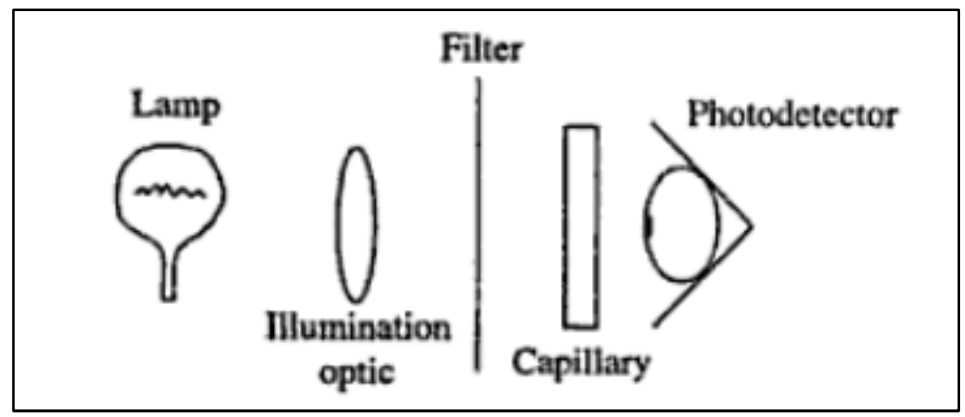

Figure 7 - Illustration of method for detection in CZE using optical means

Recently, software and computing power has reached a point where video analysis in of a section at the end of a CZE channel can be performed in real-time within the budgets of many academic programs. One such program is the LabSmith SVM software that will be explained in the materials and methods section. Recent advances in miniaturization have led to electrochemical detectors integrated with the chip that can detect concentrations on the range of 10 to $1000 \mu \mathrm{m} .{ }^{26}$

\subsubsection{Electrophoresis Theory}

In theory, a charged species moving through electrophoretic means is assumed to be a charged sphere of uniform surface charge density ${ }^{27}$. Charge of particles is usually dependent on the $\mathrm{pH}$ of the solution that the particle is suspended in. See the following amino acid for reference 


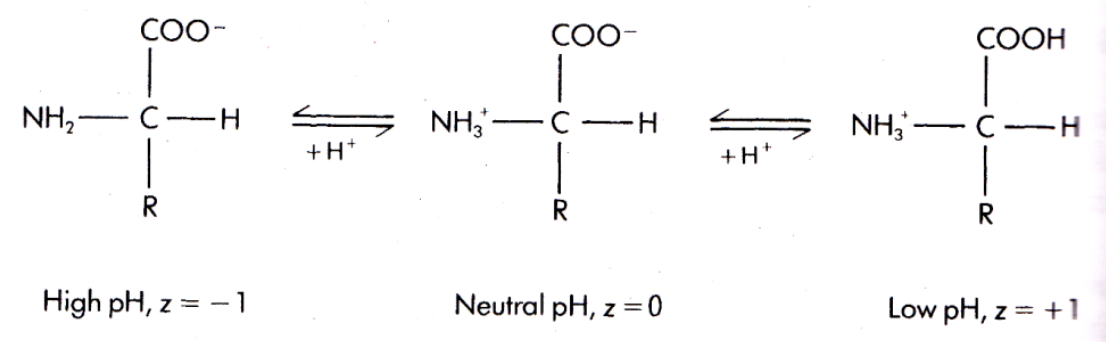

Figure 8 - Ionized forms of an amino acid as a function of $\mathrm{pH}^{27}$

At high $\mathrm{pH}$, both the carboxyl group (COO-) and the amine group $\left(\mathrm{NH}_{2}\right)$ are deprotonated, creating a net negative charge. At neutral $\mathrm{pH}$, the amine group $\left(\mathrm{NH}_{3}{ }^{+}\right)$has become charged yet the carboxyl group remains negative, creating a net neutral charge. At low $\mathrm{pH}$, the excess hydrogens in solution protonate the carboxyl group to create a net positive charge. For experimentation, carboxylated microspheres were used so it was necessary only to keep the spheres at a neutral or high $\mathrm{pH}$. The particle is also treated as a nonconductor of charge as a conducting particle would allow current to pass through it and it would then behave like a neutral particle. ${ }^{27}$

An important factor when dealing with very small particles is the Debye length. In solutions containing charged species, the electrostatic forces between particles and the species in solution will form an electric double layer, known as a Debye length or a Debye screening length commonly denoted as $\lambda_{\mathrm{D}}$ or $\kappa^{-1}$ is expressed in nanometers. ${ }^{27}$ 


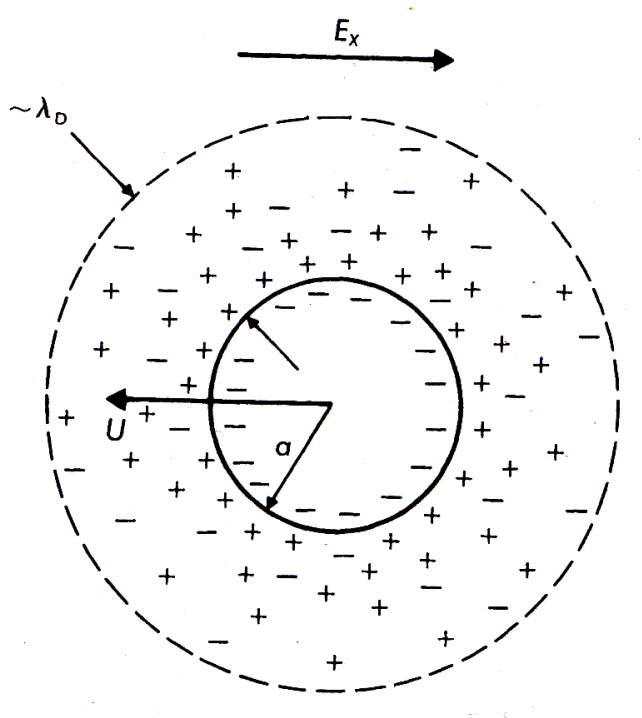

Figure 9 - Debye screening length

Particle with negatively charged surface groups with radius ' $a$ ' surrounded by Debye layer of mixed ions ' $\lambda_{\mathrm{D}}$ ' 27

During movement, the double layer around a particle will experience what is called relaxation. While the particle migrates down the channel towards its corresponding electrode, the electric double layer 'cloud' surrounding the particle attempts to strip the double layer. In the following figure, the cations are slowly being stripped away from the electric double layer and will be replaced by cations further down the channel. 


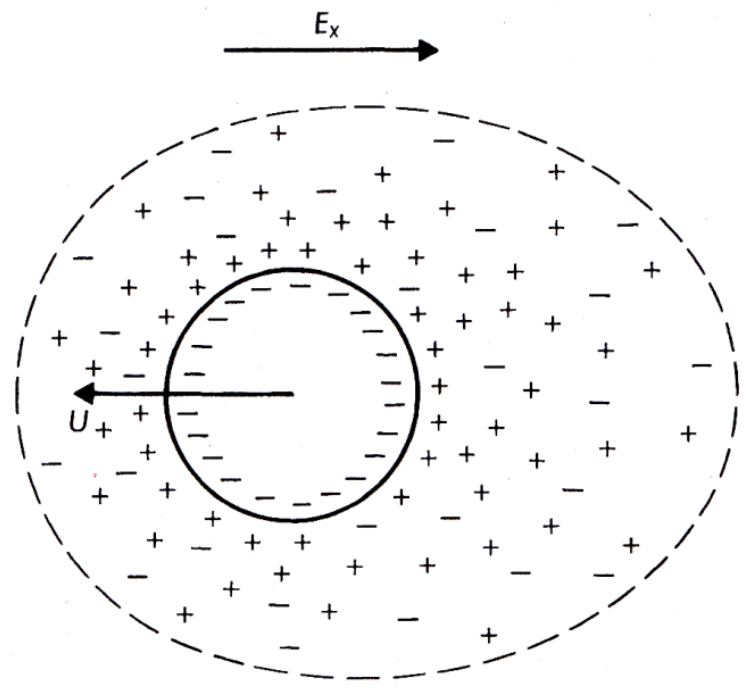

Figure 10 - Relaxation of Debye layer in a particle in motion ${ }^{27}$

This effectively retards the movement of the particle, based on the size relationship between the particle and the Debye length. Quantifying this retardation is a complex process, but for large $\left(\mathrm{a} / \lambda_{\mathrm{D}}<0.1\right)$ and small $\left(\mathrm{a} / \lambda_{\mathrm{D}}>300\right)$ Debye lengths, the effect of relaxation can be ignored. ${ }^{27}$

Calculating the Debye length around a charged sphere can be done using the following equation

Where $\varepsilon_{0}$ is the permittivity of free space, $\varepsilon_{\mathrm{r}}$ is the dielectric constant, $\mathrm{k}$ is the Boltzmann constant, $\mathrm{T}$ is absolute temperature, $\mathrm{N}_{\mathrm{A}}$ is Avogadro's number, e is elementary charge and I 
is the ionic strength of the electrolyte $\left(\mathrm{mole} / \mathrm{m}^{3}\right)$. Since this project uses the monovalent salt $\mathrm{NaCl}$ and is at $25^{\circ} \mathrm{C}$, the equation can be shortened to

$$
\lambda_{\mathrm{D}}=\frac{.304 \mathrm{~nm}}{\sqrt{\mathrm{c}_{0}}}
$$

Where $\mathrm{c}_{0}$ is concentration in moles. The Debye length was calculated to be $.02325 \mathrm{~nm}$ for all the particles as the equation is not dependent on particle size but on salt concentration as an increase in ions in the solution increases the efficiency of screening the surface charge. The Debye length is required for further calculations as the relationship between the size of the Debye layer and the radius of the particle influence the electrophoretic behavior. Based on the ratio of the particle radius to the Debye length, a different series of equations if $a / \lambda_{D}$ $>>1$ or $\mathrm{a} / \lambda_{\mathrm{D}}<<1$ although some work has been done between the ratios of $0.1>\mathrm{a} / \lambda_{\mathrm{D}}>1000$. The ratios of the 1, 5 and 10 micron particles are then 21508, 107539 and 218088 respectively, clearly putting the ratio well over 1000. The next important step in calculating electrophoretic velocity is the calculation of the net charge of the particle. ${ }^{27}$

Theoretically calculating the net charge of a particle is beneficial to researchers and manufacturers of biochemistry products as well as for theoretical electrophoretic mobilities. Once a theoretical or experimental surface group density can be determined, the net charge of a particle of a given size can then be calculated. For example, Spherotech labs reports that the carboxyl group density on the surface of their microspheres is roughly $320000 / \mu \mathrm{m} 2$. The surface charge of a particle is can be related to its net charge, or z-potential by use of the Grahame Equation. ${ }^{28}$ 


$$
\sigma=\sqrt{8 c_{0} \varepsilon \varepsilon_{0} k_{B} T} \cdot \sinh \left(\frac{e \varphi_{0}}{2 k_{B} T}\right)
$$

Where $\mathrm{c}_{0}$ is the concentration of the concentration in moles $/ \mathrm{m}^{3}, \varepsilon$ is the permittivity, $\varepsilon_{0}$ is the dielectric constant, $\mathrm{k}_{\mathrm{B}}$ is Boltzmann's constant, $\mathrm{T}$ is temperature in Kelvin, $\mathrm{e}$ is the number $2.718 \ldots$, and $\varphi_{0}$ is the concentration of surface groups in groups $/ \mathrm{nm}^{2}$.However, because this application is using low potentials, the sinh operator can be expanded into a series and the first term can be ignored, leading to

$$
\sigma=\frac{\varepsilon \varepsilon_{0} \varphi_{0}}{\lambda_{D}}
$$

Where $\lambda_{D}$ is the Debye length. ${ }^{28}$

\subsubsection{Electrophoretic Mobility}

With particles moving within a liquid, drag becomes a factor. Particles in liquids with low Reynolds numbers, common in microfluidics, will experience a drag force per the following equation

$$
F=6 \pi \eta R v
$$

Where $\mathrm{F}$ is the force due to drag that points in the opposite direction of travel, $\eta$ is the viscosity of the liquid, $\mathrm{R}$ is the radius of the particle and $\mathrm{v}$ is the velocity. The particles accelerate to a certain electrophoretic speed and then maintain that velocity, so drag must be equal to electrophoretic force. The velocities then become

$$
v_{i}=\frac{Q E}{6 \pi \eta R v} \text { and } v_{o}=-\frac{Q E}{6 \pi \eta\left(R+\lambda_{D}\right)}
$$


Where QE and -QE are the applied forces for the particle and the Debye layer respectively. The net velocity is the sum of the two equations

$$
v=\frac{Q E \lambda_{D}}{6 \pi \eta R^{2}}\left(1+\frac{\lambda_{D}}{R}\right)^{-1}
$$

Because the Debye layer is so much smaller than the particle radii, the following equation for velocity can be shortened to the version on the right

$$
U=\frac{2}{3} \cdot \frac{\zeta \varepsilon\left(1+\frac{a}{\lambda_{D}}\right) E_{x}}{\mu} \approx \frac{2}{3} \frac{\zeta \varepsilon E_{x}}{\mu}
$$

Where zeta is the net potential of the particle as calculated in the Grahame equation, $\varepsilon$ is permittivity, $E_{x}$ is the electric field strength and $\mu$ is the viscosity of the solution. $U$ is the term for electrophoretic velocity and is usually expressed in units of $\frac{\mu m}{s}$. The $\frac{2}{3}$ factor at the front comes from the ratio of the radius to the Debye length. When $a / \lambda D<<1$, that factor becomes $\frac{1}{2}$. Alternatively, the electrophoretic velocity can also be referred to as the 'electrophoretic mobility', the relation of U/Ex or the velocity in relation to the applied electric field. $\frac{\mathrm{cm} \cdot \mu \mathrm{m}}{V \cdot S}$. Some of the variables in the equations must be calculated based on the testing conditions. ${ }^{27}$

The zeta potentials for the 1 and 10 micron spheres were calculated using the surface group density data from the manufacturer. Bangs Labs, the manufacturer of the 5 micron spheres did not keep data for the surface group density so data from Spherotech's 5 micron spheres was used. The value of the dielectric constant for the $1 \% \mathrm{NaCl}$ solution was taken from 
literature as being 78.7..$^{30}$ The Grahame equation was used to calculate the following values for net charge.

Table 1 - Carboxyl group surface density and net potential for microspheres

\begin{tabular}{|l|r|r|r|}
\hline & \multicolumn{1}{c|}{ Micron } & 5 Micron & 10 Micron \\
\hline $\begin{array}{l}\text { Carboxyl groups per } \\
\text { particle }\end{array}$ & $2.19 \mathrm{E}+08$ & $3.45 \mathrm{E}+08$ & $4.00 \mathrm{E}+08$ \\
\hline Surface area in $\mathbf{n m}^{\wedge} \mathbf{2}$ & $3.14 \mathrm{E}+07$ & $7.85 \mathrm{E}+08$ & $3.15 \mathrm{E}+09$ \\
\hline $\begin{array}{l}\text { Carboxyl groups per } \\
\mathbf{n m}^{\wedge} \mathbf{2}\end{array}$ & 0.06956 & 0.0439 & 0.12681629 \\
\hline Net Potential $(\mathbf{V})$ & 0.73410242 & 0.463552 & 0.1338272 \\
\hline
\end{tabular}

The solution contained a $4 \%$ concentration Tween, a high viscosity liquid used to prevent clumping of microspheres and necessitated used of the Refutas equations to determine the overall viscosity of the liquid. Each VBN (viscosity of blend) value must be found for each fluid then added together based on their volume fraction, $x$. The sum must then go through the inverse of the first equation to return the viscosity of the blend.

$$
\begin{gathered}
V B N=14.534 \cdot \ln [\ln (v+0.8)]+10.975 \\
\sum x_{n} \cdot V B N_{n} \\
v=\exp \left(\exp \left(\frac{V B N_{\text {blend }}-10.975}{14.534}\right)\right)-0.8
\end{gathered}
$$

The average value of viscosity of $300 \mathrm{cp}$ at $25^{\circ} \mathrm{Cfor}$ Tween was used. The value for the $1 \%$ $\mathrm{NaCl}$ solution at $25^{\circ} \mathrm{C}$ was taken from literature to be 0.9042 (Lang Jun). With a $4 \%$ Tween/96\% $\mathrm{NaCl}$ solution the overall viscosity was taken to be .997 . 


\subsubsection{Electroosmotic Flow}

Another effect worth mentioning is electroosmotic flow or the flow of a conductive solution when an electric field is applied across it. The charged surfaces of the capillary walls attract the positive ions in the water and repel the negative ions. This will cause the fluid to flow towards the negative potential. For the purposes of modeling, PMMA was taken to have a zeta potential of $-13 \mathrm{mV}$ according to literature. ${ }^{29}$ Electroosmotic flow can be modeled with the following equation.

$$
\mu_{o}=-\frac{\epsilon \zeta}{\mu}
$$

Where $\mu_{\mathrm{O}}$ is the electroosmotic mobility of the fluid, $\varepsilon$ is the permittivity, and $\mu$ is the viscosity of the fluid. All of these variables were kept the same form the electrophoresis section, which yielded an electroosmotic mobility of -1.026 which factors in as somewhat insignificant compared to the electrophoretic velocities of the spheres. The negative value of the electroosmotic velocity means it acts against the flow of the particles. Increasing electric field yield increasing electroosmotic velocities. Total flow in an electrophoretic separation can be written as

$$
\mu_{T}=\mu_{E}+\mu_{O}
$$




\subsubsection{Final Calculations}

With the viscosity and zeta potential calculated, the electrophoretic velocity could be calculated. Because the experimentation uses several different voltages, the electrophoretic mobility will instead be used.

Table 2 - Electrophoretic mobility of microspheres

\begin{tabular}{|l|c|c|c|}
\hline & $\begin{array}{l}\mathbf{1} \\
\text { micron }\end{array}$ & $\begin{array}{l}\mathbf{5} \\
\text { micron }\end{array}$ & 10 micron \\
\hline $\begin{array}{l}\text { Electrophoretic Mobility } \\
\text { U/Ex }\end{array}$ & 38.63 & 24.4 & 12.1 \\
\hline Total Ep+Eo & 37.60 & 23.37 & 11.07 \\
\hline
\end{tabular}

These values will be calculated against the experimental results in the Discussion section.

\subsubsection{Laser Processing}

Laser (standing for Light Amplification by Stimulated Emission Radiation) technology has been around for roughly fifty years and can be seen in a wide variety of fabrication, biological, and chemical applications outside of this project. Laser power is commonly referred to in the form of watts or watts $/ \mathrm{cm}^{2}$, as you will see in this paper.

\subsubsection{Dynamics of Laser Power}

The machine used for this project was the Universal Laser Systems X2-660 120W laser system in the Mustang '60 machine shop located in Bonderson Engineering. Because this is not a "constant-on" laser system, the laser can be pulsed up to 1000 times per inch to create a finer image and down to 500 pulses per inch for rough cuts. Because of the fine features used in this project, the 1000 pulses per inch setting were used for every cut.

Lasers typically have a Gaussian distribution of power with the highest concentration of cutting power at the center of the beam. As the laser cuts through material and the pulses 
overlap, it effectively sweeps a 2-D profile through the material. The dimensions of this profile are dependent on laser power and wavelength.

\subsubsection{Vaporization and Ejection of Molecules}

When the laser beam hits the surface of the workpiece, it imparts energy that first melts then decomposes the material. The rapid rise in temperature at that zone then ejects the materials out of the void as shown in the following figure

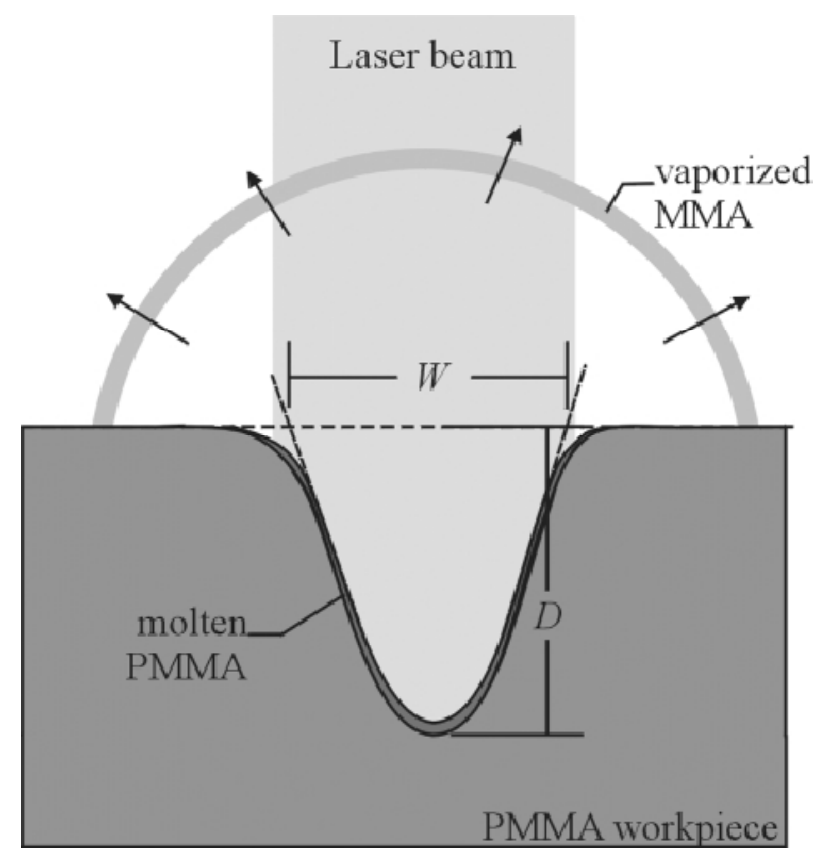

Figure 11 - Dynamics of laser ablation in PMMA ${ }^{31}$

The penetration depth of the laser is dependent on the bonds within the material, both the monomer bonds and the H-bonds between strands of MMA. ${ }^{32}$ Not all of the material is ejected, however, as the portion of the material that has reached the temperature required for liquidation of PMMA yet not received enough. Some material that is ejected redeposits outside the width of the laser beam and creates features on top of the surface of the PMMA, as shown in the following figure. This is very beneficial for microfluidics applications. The 
redeposited material acts as a seal for the channel when a backing is applied to the chip. Because the thermal diffusivity of the PMMA is low, the depth of the channel at the pulses is fairly constant once the laser has begun cutting -i.e. the workpiece does not slowly rise in temperature causing cuts of increasing depth as the cutting time increases. The only factors causing inconsistencies within channel depth are pulse overlapping, material redeposition and cuts at the beginning of channels.

Pulse overlapping is related to the PPI, or pulses per inch, setting on the laser. The maximum setting is 1000 PPI meaning the separation between pulses is roughly 25.4 microns (dependent on machine consistency). From the profilometer results we can assume that beam width is related to laser power. For consistency with the final chip design 5\% power setting cutting will be referred to from here on.

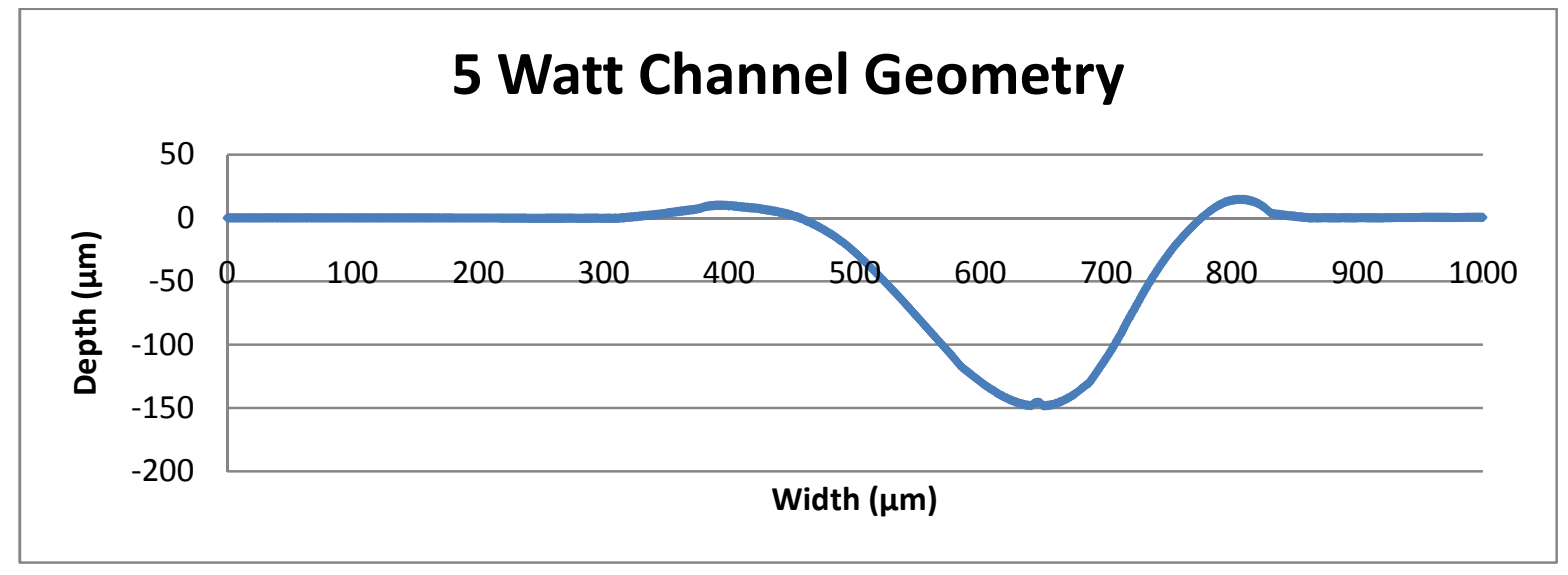

Figure 12 - Profile of a laser-cut channel in PMMA using the 5\% power setting. Width is $319 \mu \mathrm{m}$ and $148 \mu \mathrm{m}$ depth. Note the $X$ and $Y$ axes are not to scale.

The channel width is $319 \mu \mathrm{m}$, which we can assume is the effective beam width for this power setting and material. Therefore, we can assume that with a 25.4 micron beam stepover the channel will be fairly consistent in machining standards. The redeposition of 
the material on the edges will lead to the most inconstancies. Snakenborg et al found and graphed a relationship between laser speed, power and depth. The depth data from the laser cutting trials largely matched the data from the paper. ${ }^{33}$

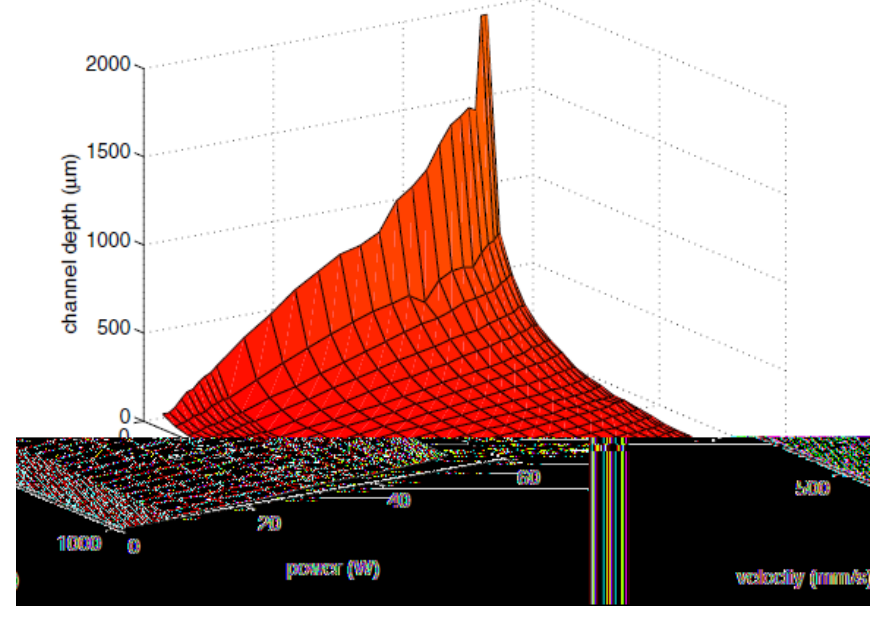

Figure 13 - Graphical relationship between power, velocity and channel depth of infrared laser. ${ }^{33}$

\subsubsection{Directionality of Laser Cutting}

Taking into account the dynamics of laser cutting and the ejection/redeposition of the material, the designer of a microfluidic chip must take into account the order of etching steps when fabricating a very precise chip. When cutting a channel across another, at the junction between those two channels the edges of the second channel have the possibility of creating a blockade in the first. The following figure will explain this further. 


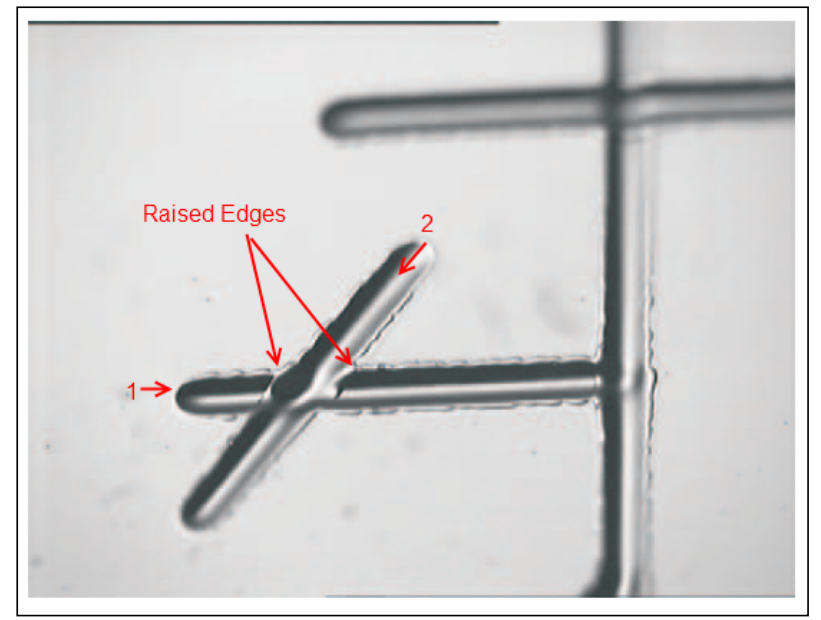

Figure 14 - Photograph of channels in PMMA creating a T-junction. The diagonal line was created after the horizontal ${ }^{33}$

As indicated by the red numbers and arrows, the horizontal cut came first, followed by the 45 degree cut. The redeposited material can be seen on the edges of both the channels, but at the junction between the two some material has melted and resolidified without being ejected from the channel. This may not be a problem for very small molecules, but in channels that are not very deep these raised edges may obstruct cells or even fluid flow completely. When creating junctions between channels, this effect must be taken into account. At a simple T-junction in the figure below, 4 different combinations of cut patterns were used to create a similar junction.

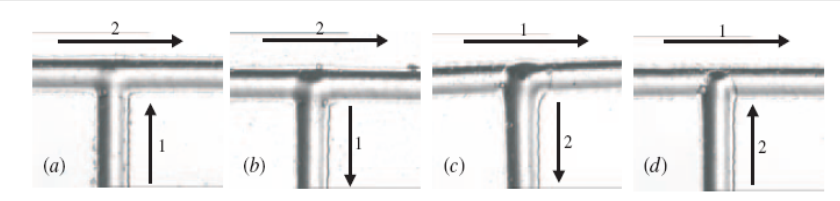

Figure 15 - Directionality associated with sequences of laser cuts in PMMA. Numbers indicate cut sequence and arrows indicate direction ${ }^{33}$ Laser processing ejects material out of the area during the ablation process. Most of the material redeposits on the lip of the channel, but some will redeposit on the surface of the 
material several millimeters away from the location of the photoablation. While these particles may be on the range of 10 s to 100 s of nanometers in diameter, they still have the chance to partially obstruct channels and increase the surface area for proteins to adsorb to. For this fabrication process, the ablation-intensive process to cut the raw plexiglass sheet into individual chips and cut the holes for the tubing to make a functional chip was performed first. After this cutting process, the chips were left untouched in preparation for the capillary cutting process.

Using the AutoCAD software it is relatively easy to line up the microchannels with the through-holes used to connect tygon tubing to interface with the chip. Using different colors for the geometries in AutoCAD, specific laser powers can be applied. An example of this is shown in the following figure.

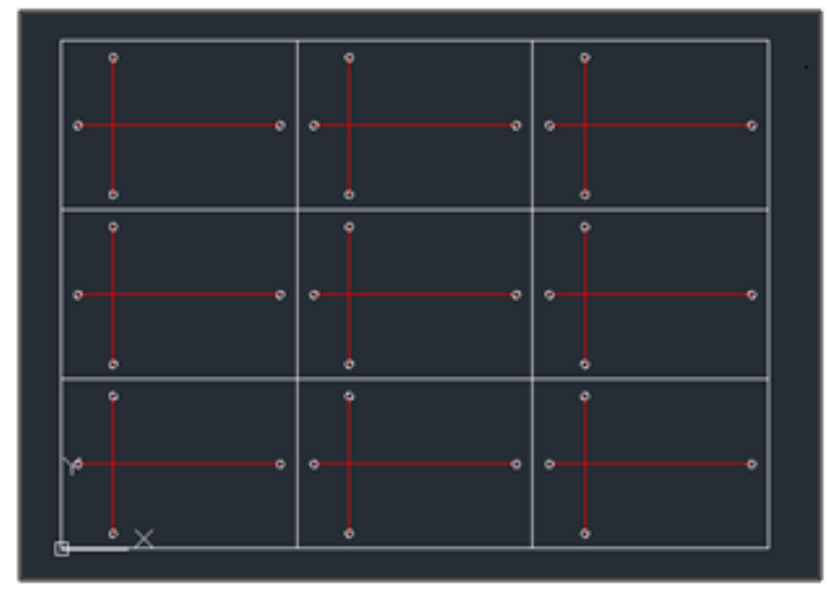

Figure 16 - AutoCAD draft of electrophoresis chips. High-power cuts are white and are meant to cut completely through the PMMA. Red lines are for etching the material for channels 


\section{Methods and Materials}

This section details the microfluidic chip manufacturing methods that were attempted during the course of the project. Many of the methods are derived from examples in literature and were pursued in parallel paths to determine the most promising method at each step, e.g., the different chip geometries were fabricated during the same day and tested after construction. The ideas that did not make it to the final product are by no means patently unsuccessful; they simply did not show as much promise in regards to experimentation or manufacture.

\subsubsection{Geometry and Drafting}

The AutoCAD drafting software allows for precise measurement and alignment of geometries between files with its command-line interface. This is excellent for the 2dimensional computer modeling for laser processing. By using the Universal Laser Systems' provided plotter print driver from AutoCAD, a user can command the laser cutter to follow the lines in the drawing with an accuracy of 1/1000" or 25.4 microns according to the manufacturer's specifications. Laser power can be associated with line color on the printing options so multiple laser powers can be used in a single run, especially beneficial for trials for quantifying laser power output in channel depth.

\subsubsection{Geometry}

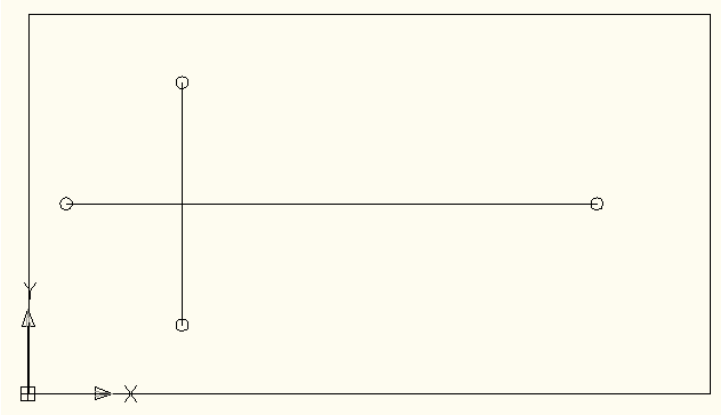

Figure 17 - Standard CZE chip design. Horizontal channel length is $85 \mathrm{~mm}$, vertical channel length is $55 \mathrm{~mm}$. Vertical channel is $25 \mathrm{~mm}$ from the end of the horizontal channel. Holes are $.375 \mathrm{~mm}$ in diameter 
The initial design used for several sets of laser cutting was the t-design seen above. Starting from the top and moving clockwise the holes are used for the following: sample injection port, buffer injection, waste port, buffer injection. Many literature sources used this simple geometry with varying lengths and distances. ${ }^{34,35,36}$ The lengths and distances associated with this geometry were adapted from the following diagram and authors. The final lengths of the channels were $85 \mathrm{~mm}$ for the long and 55 for the short. The fluids will enter through the ports at both ends of the horizontal channel and the topmost port. Through laminar flow, the buffer will 'pinch' the sample and shuttle it to the waste port to prevent contamination of the analysis channel. The electrodes will be placed at the ends of the horizontal channel with the positive lead at the rightmost port. The pressure driven flow will be turned off and the sample left at the junction of the two channels will move down the channel to the right and fractionate.

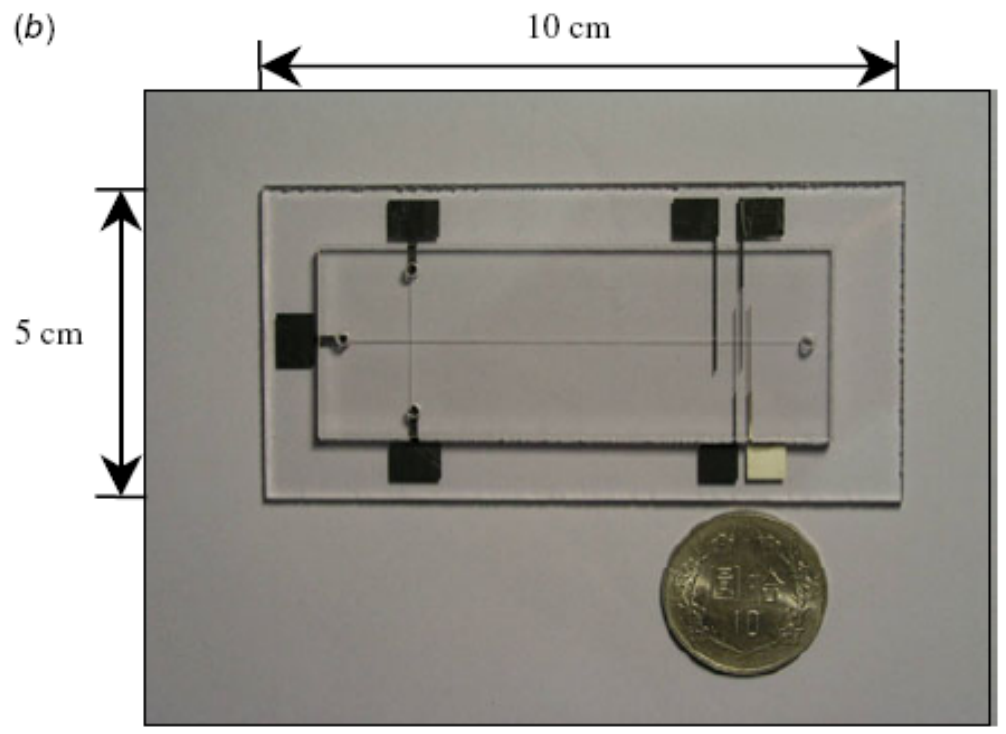

Figure 18 - Example CZE simple design ${ }^{43}$ 


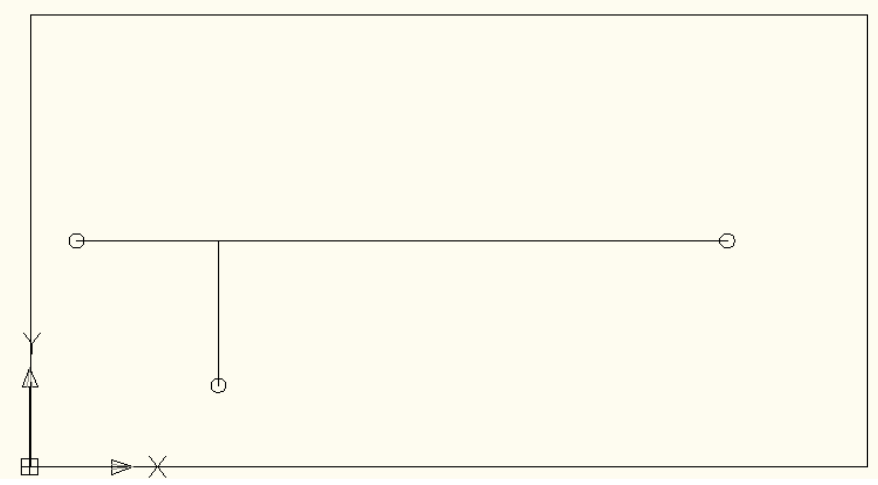

Figure 18 - Standard CZE chip design. Horizontal channel length is $85 \mathrm{~mm}$, vertical channel length is $27.5 \mathrm{~mm}$. Vertical channel is $25 \mathrm{~mm}$ from the end of the horizontal channel. Holes are $.375 \mathrm{~mm}$ in diameter

This simpler design adapted from literature features only three inputs and was used as a possible design to create a "plug" of sample that could be moved down the channel. ${ }^{37,38}$ The length of the plug would be the distance from the intersection of the two channels to the left-most input port. The dimensions for this chip are similar to the previous design, except the vertical channel is $22.5 \mathrm{~mm}$. Starting from the left and moving clockwise, ports are used for the following: waste port, buffer injection, sample injection port. The fluids will enter in the buffer and sample injection ports and exit out the waste port. After the sample 'plug' has filled the leftmost portion of the horizontal channel, the pressure-driven flow will stop and the electrodes will be turned on. The electrodes will attach to the two ports in the horizontal channel with the positive electrode connected to the rightmost port and the fractionation will happen from left to right. 


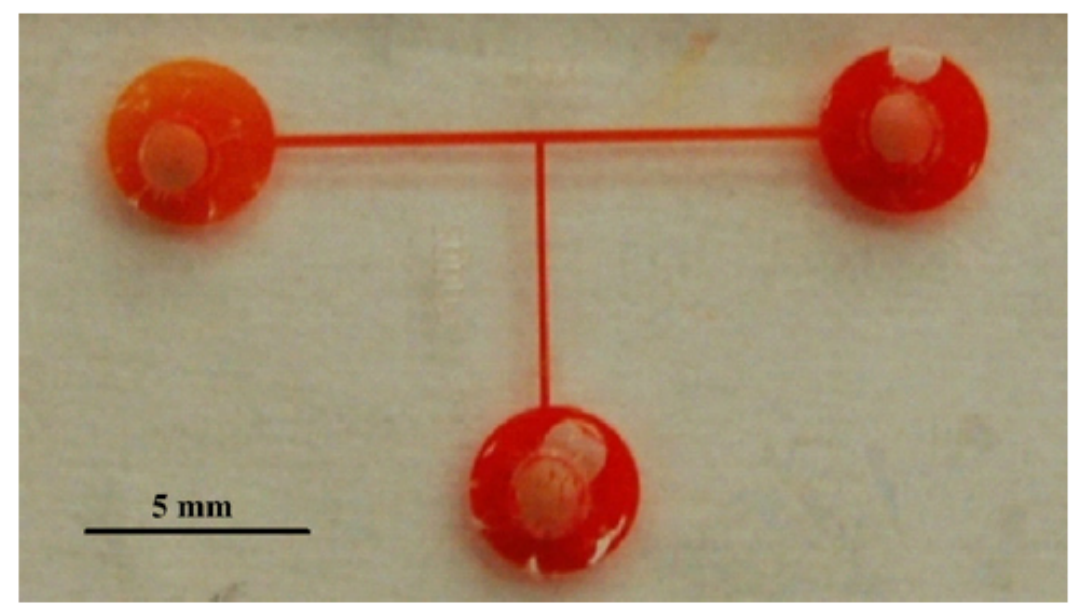

Figure 19 - T-channel design for CZE ${ }^{37}$

There are additional ways to create plugs combining the previous two ideas and ideas from literature. $^{39,40}$

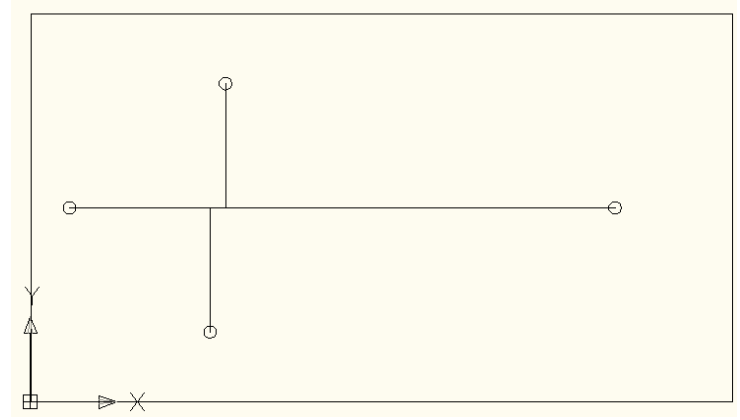

Figure 20- Step design for creating a sample "plug". Horizontal channel length is $85 \mathrm{~mm}$, vertical channel lengths are $27.5 \mathrm{~mm}$. Bottom vertical channel is $25 \mathrm{~mm}$ from the end of the horizontal channel, top vertical channel is $3 \mathrm{~mm}$ to the right. Holes are $.375 \mathrm{~mm}$ in diameter.

Similar to the previous geometry in that this design would create a sample "plug" that could be moved down the channel. This design showed the most promise, as will be discussed in the results section. Sample ports are identical to the first design, starting with topmost port 
and going clockwise they are sample injection, buffer injection, waste and buffer injection. The length of the sample plug can be adjusted with the distance between the vertical channels, indicated as ' $2 \mathrm{~h}$ ' in the following diagram. The chips manufactured with this geometry were the same as

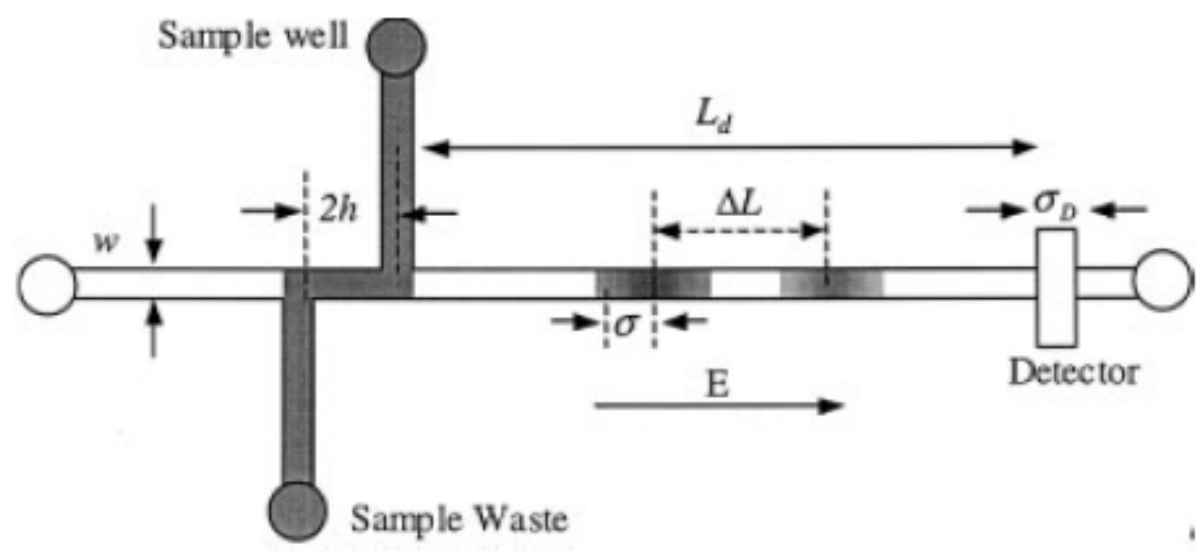

Figure 21 - Design for 'step' CZE chip. ${ }^{40} \mathrm{~A}$ chip with this geometry allows for the creation of a sample plug that can be fractionated down the channel

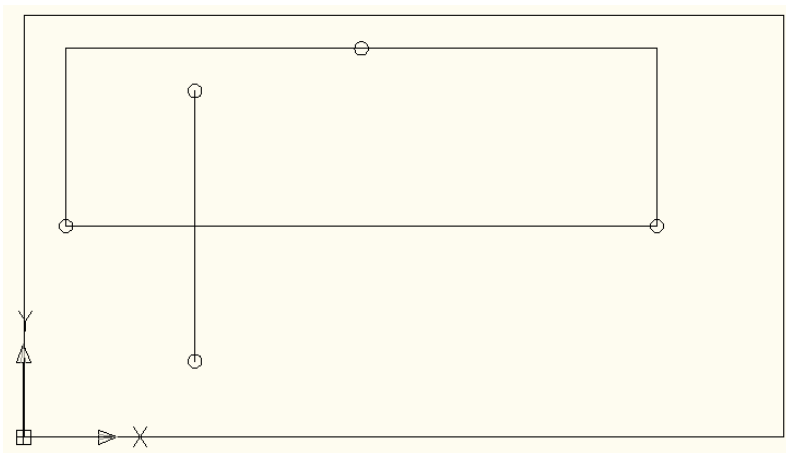

Figure 22 - Single buffer input design. Horizontal channel lengths are $85 \mathrm{~mm}$, vertical channel length is $55 \mathrm{~mm}$. Vertical channel is $25 \mathrm{~mm}$ from the end of the horizontal channel. Hole on upper horizontal channel is $\mathbf{4 2 . 5} \mathrm{mm}$ from the end of the channel. Holes are $.375 \mathrm{~mm}$ in diameter

The point of this design is to have a single injection port so that only one syringe pump with a buffer is used and no balancing of the injection rates is required. The purposes of the 
holes are as following starting from the left and moving clockwise: electrode with no flow, sample injection, buffer injection, electrode with no flow, waste port.

Other chip geometries existed in literature that might serve useful for future student projects but were not used for this project.

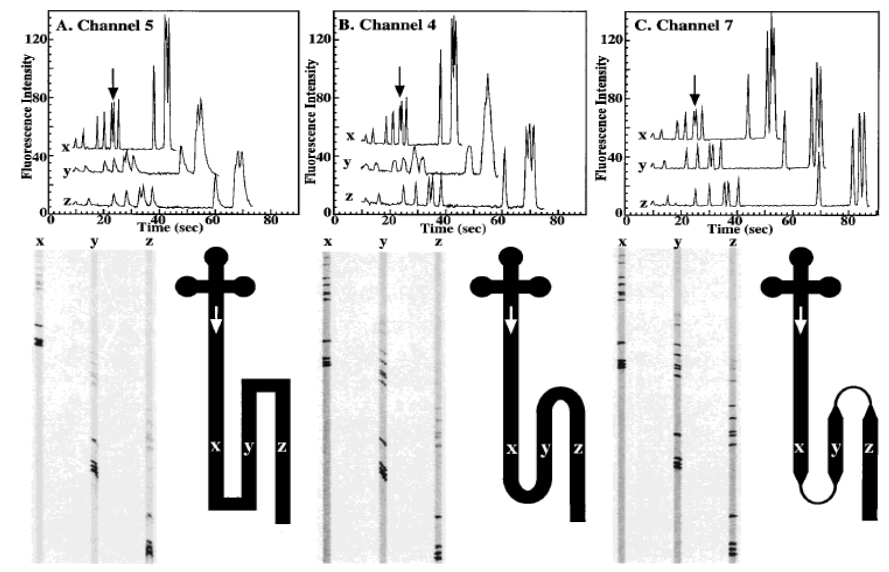

Figure 23 - Optimization of serpentine channels using different geometries. ${ }^{42}$

A method commonly used in literature to lengthen channels within a compact space is creating serpentine channels. Because the electric current carries through the length of the channel regardless of shape, this effectively lengthens the channel several times over. One of the key issues with this sort of geometry is fluid flow through the channel, as is being addressed in the figure and paper above as fluid dynamics come into play with these geometries. Observing the corresponding output graphs above the chip geometries, the "C" geometry with pinching between the curves yields the highest resolution separations at the cost of a longer timespan. ${ }^{42}$ The use of a fairly long serpentine channel is shown in the following figure. 


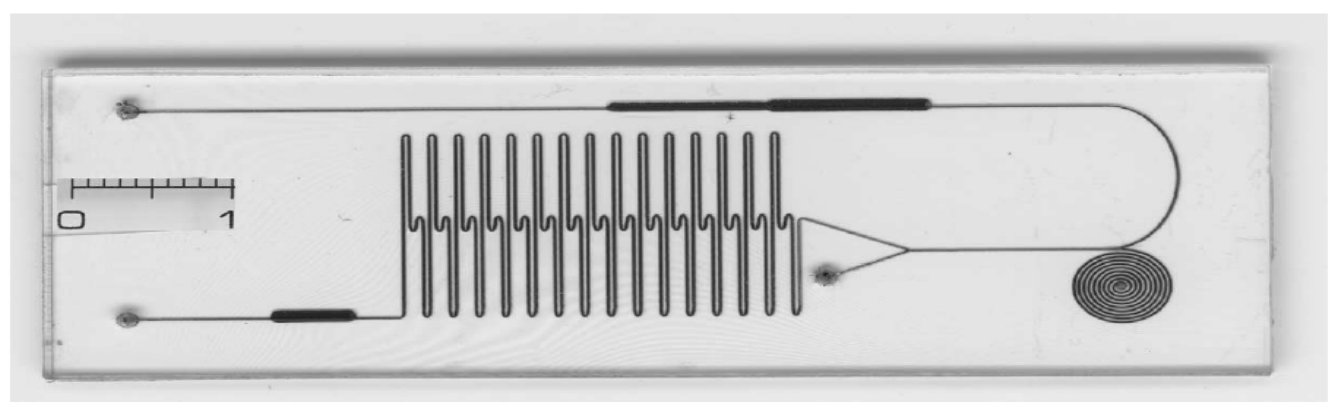

Figure 24 - Serpentine separation channel CZE design. Scale bar is $1 \mathrm{~cm}^{53}$

Application of the serpentine channel design by Cheng et al.. Use of this channel design increases the effective length of the separation channel by an order of magnitude. These chip designs are for optimization purposes and because this thesis was based on implementing a new method of manufacture, it was deemed that optimization should be a project for future students.

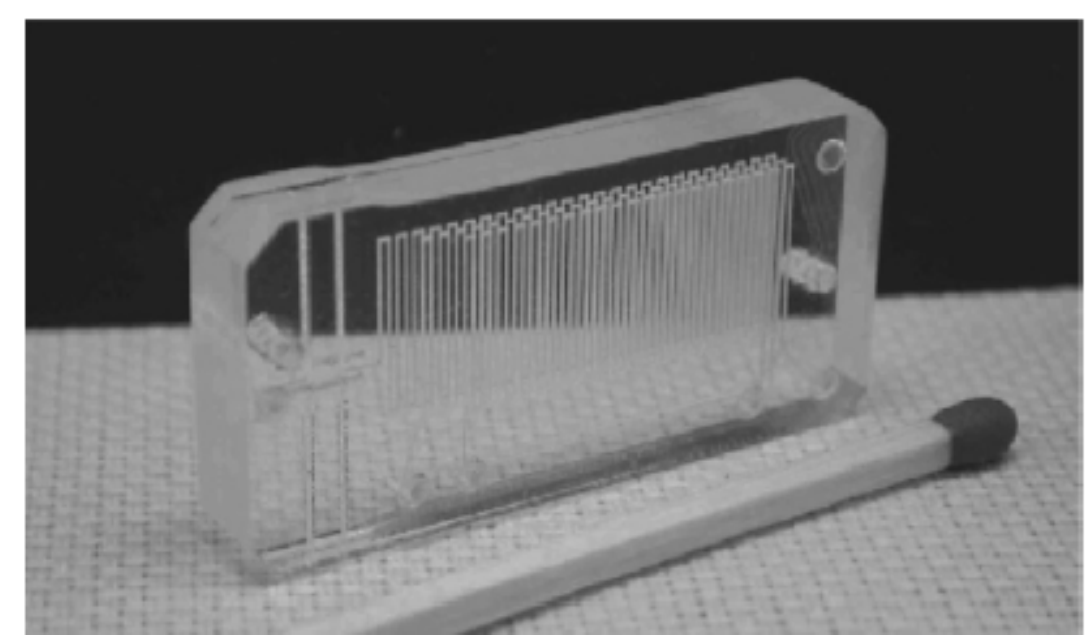

Figure 25 - 3-D PMMA chip design created to mix a sample with three different reagents and perform a separation. A standard match is shown for size reference

The accuracy associated with laser etching can be used to create 3-dimensional microfluidic chips. Using bonding methods discussed in another section, a stack of chips can be bonded 
together to created channels that flow in 3 dimensions. Laser-cutting landmarks such as through-holes can be used to align the different layers of chips using pins or machine screws.

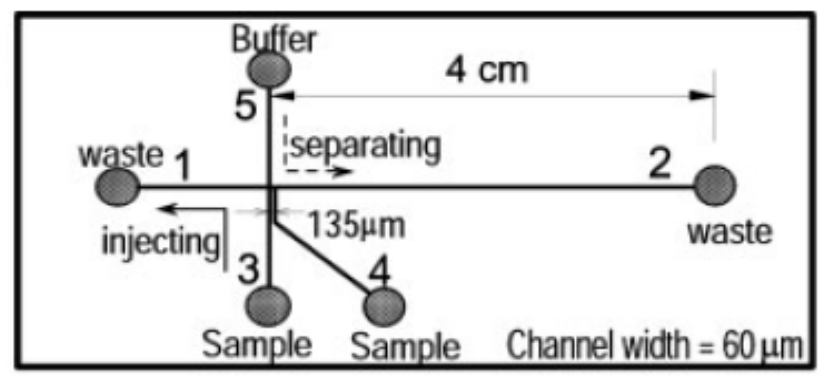

Figure 26 - CZE chip geometry for multiple sample injections

Sometimes in CZE experimentation it is necessary to inject multiple samples into a chip in different ports. This can easily be accomplished using laser etching as well. For the purpose of this experimentation, only one injection port was required.

\subsubsection{Channel Facing Down vs Up}

Most PDMS chips are created so the features are on the bottom of the chip so an inverted microscope only has to focus through the glass slide the chip is attached to. The tubing that creates the connection between the syringes and the chip must approach from above the chip so as to not cause interference. In the following designs, the two layer PMMA design and the raster-cut reservoir designs require the inverted microscope to focus through the bottom of the lasercut channels in what will be referred to as an 'upward-facing channel' in that the opening of the channel faces up. This design should be avoided by future researchers. Light produced by the inverted microscope will be scattered by the rough edges of the channels unless they undergo post-treatment smoothing. If the chip is etched, covered, and inverted for study, this will be referred to as a 'downward-facing channel'. A downwardfacing channel is preferred, as the microscope must only focus through the channel cover 
material and direct light is not scattered by the rough channel. A downward facing channel also allows for larger tolerances when inserting connection tubing as shown in the following diagram.

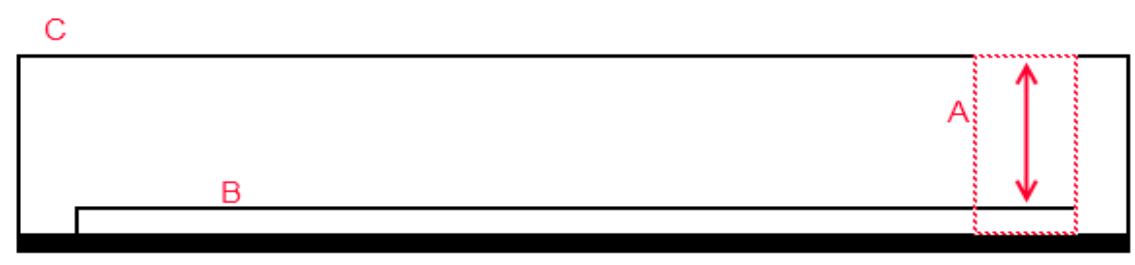

Figure 27 - Tolerance for tygon tubing insertion. Through-hole is indicated by A, Channel is indicated by $B$ and the PMMA boundary is indicated by $\mathrm{C}$. The solid black line indicates the sealing boundary between the channel and the outside.

The circular ports at ends of the channels are commonly referred to as reservoirs, as they

contain a larger amount of sample than what is held in the channels. Also, the tubing connections used to interface with microfluidic chips are commonly many times larger than the microfluidic channels. 4 different reservoir designs were tested during the chip manufacturing stages.

\subsubsection{Chip Interfacing}

The features on microfluidic chips are very small but there must be an interface between them and other experimental equipment such as syringe pumps and voltage sources that bridge the micro and macro scales. Tygon tubing is a widely using material in biomedical science as it is stable at a range of temperatures and is nonreactive to many chemicals. All tubing used in chip construction was of 1/16" OD and 1/32" ID and cut with a special tubing cutter to ensure clean perpendicular cuts were created. Tygon tubing was connected to the 23 gauge needles on the injection syringes per the following figure. 


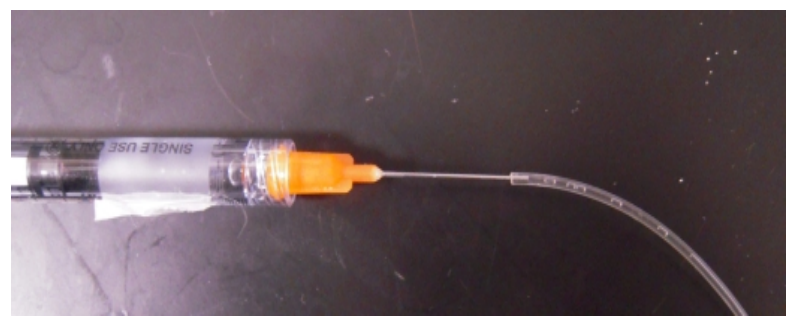

Figure 28 - Tubing connection created with injection syringe and 23 gauge blunt needle

During chip construction it was found to be easiest to keep tygon connections that were adhered to the chip less than 3 inches to decrease the amount of time to clear fluids from previous experiments. In order to connect the section of tubing leading from the syringe and the section of tubing leading from the chip, 23 gauge 304 stainless steel hypodermic tubing was used. Tubing is a difficult material to cut with hand tools without deforming it so the tubing was cut using a razor blade. The tubing was cut by holding a razor blade firmly against the top of the tubing in a perpendicular fashion. The tubing was then rolled underneath the razor blade until the cut section could be easily snapped off. The hypodermic tubing was then inserted into the tygon tubing, forming a very tight seal.

\subsubsection{Reservoir Design}

All adhesives used during the construction of chips were Loctite Control Gel cyanoacrylate. When designing the microfluidic chips that were to use PMMA as both the sacrificial material for the channel and the channel cover for the microwave and oven bonding methods, the idea was to have the microfluidic channels etched into one PMMA sheet and cut out a cover with reservoirs/tubing connectors of the same size and bond the two together. 

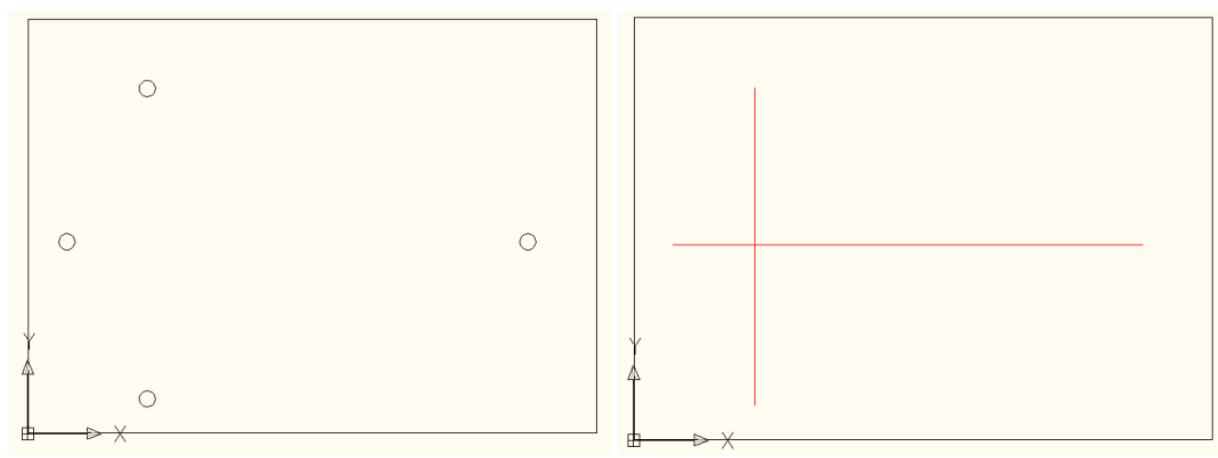

Figure 29 - CAD model for laser chip cutouts and through-holes for integration (L) with tubing and syringe pumps and corresponding channel etches $(\mathbf{R})$

Using AutoCAD software it is very simple to align the reservoirs with the channels so the chips can be cut out to the same size and bonded. One method to create multi-layered chips is to draw out the projection of the layers of the chip in AutoCAD and only associating laser powers with the colors of the lines that need to be cut. Another method is to again draw out the entire chip and selectively delete portions and save those as individual files to be cut separately as shown below.

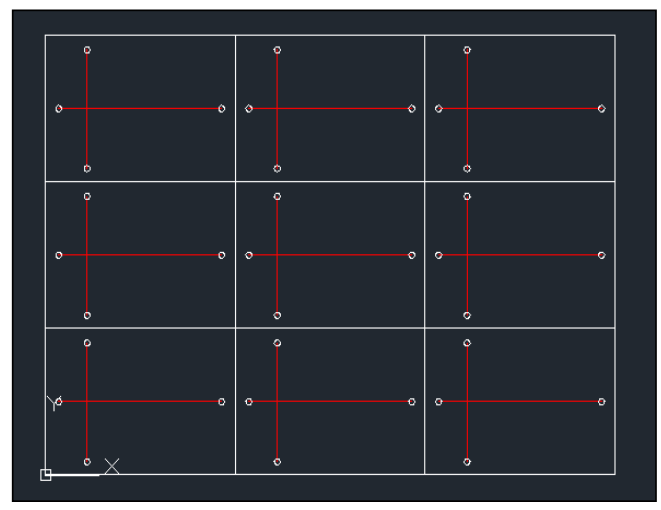

Figure 30 - Complete mockup of two-layered chip design. Channels are shown in red and cutouts for through-holes and chip edges 


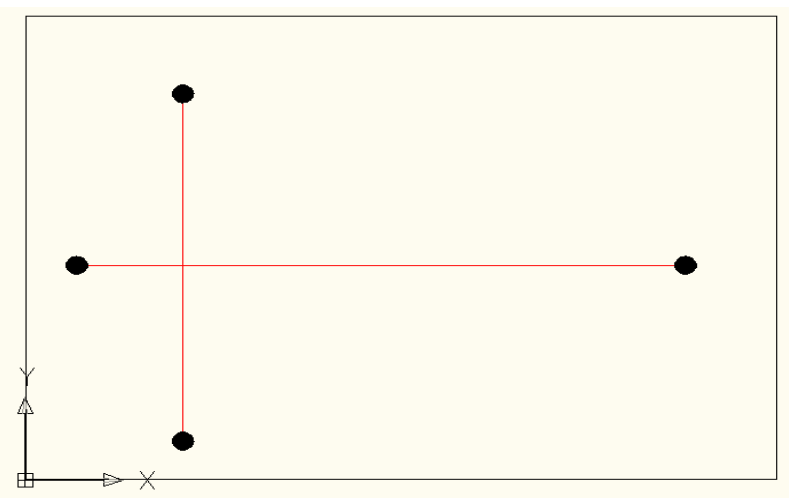

Figure 31 - Hatch designs for raster cut reservoirs

A concept to create reservoirs that were wells in the chip instead of througholes was tested as well. The laser cutter is capable of making two types of cuts: vector and raster. By setting line thicknesses in AutoCAD to $0.00 \mathrm{~mm}$, the plotter that directs the laser cutter will make a single pass or vector cut into the material. By making line thicknesses any greater than $.025 \mathrm{~mm}$ or 25 microns, the laser cutter will make two passes directly next to each other as the minimum stepover for the laser cutter is .001 ” or 25.4 microns. This is called a raster cut and is what most associate with printing documents as the printer will create a document line by line. Using the 'hatch' tool in AutoCAD, the user can create a design for the laser cutter to raster. The laser cutter will take multiple passes over the raster areas and etch into the material according to the design. This is helpful for future students attempting to create chambers in PMMA with the laser cutter. However, inserting the tubing to a depth less than the channel depth and achieving proper sealing with a tape backing proved to be insurmountable.

Following the designs of previous students working with PDMS, the following design was created so the hypodermic needle tubing could fit directly into the PMMA and adhesive could be used to secure it. 


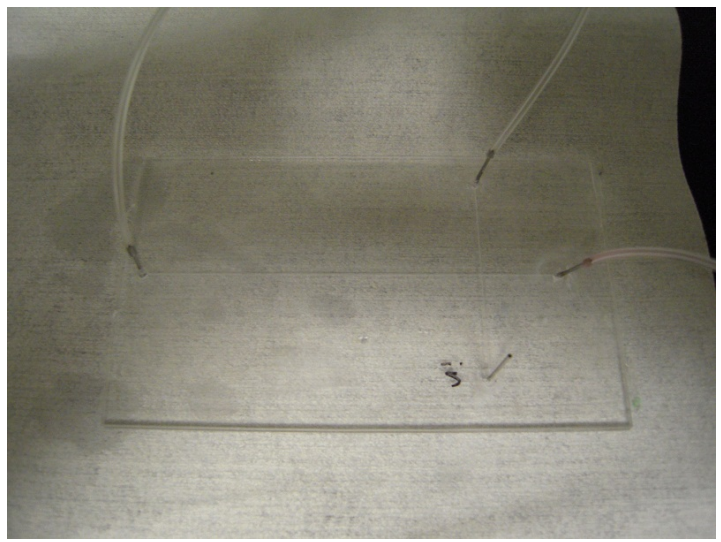

Figure 32 - 23 Gauge through-holes for direct connection with hypodermic needle tubing

This design ran into the power distribution problem commonly associated with laser cutters in very fine applications. The power of the laser cutter is most intense in the middle of the beam and falls off rapidly approaching the edge of the beam. When creating a hole that is about $1 \mathrm{~mm}$ in diameter, the beam's power distribution problem becomes apparent. The roughly conical channel ablated causes the OD of the throughhole to shrink as the depth increases. At the top the hole can be 23 gauge or $1.1 \mathrm{~mm}$, but at the bottom it is closer to $0.9 \mathrm{~mm}$ so the hypodermic tubing cannot be set into the channel firmly as there is only a small section of PMMA that is 'holding' the hypodermic tubing in place being that PMMA is a very stiff material. The adhesive was not able to hold the tubing in place to the point to make this a viable design. Below is a figure demonstrating this effect. 


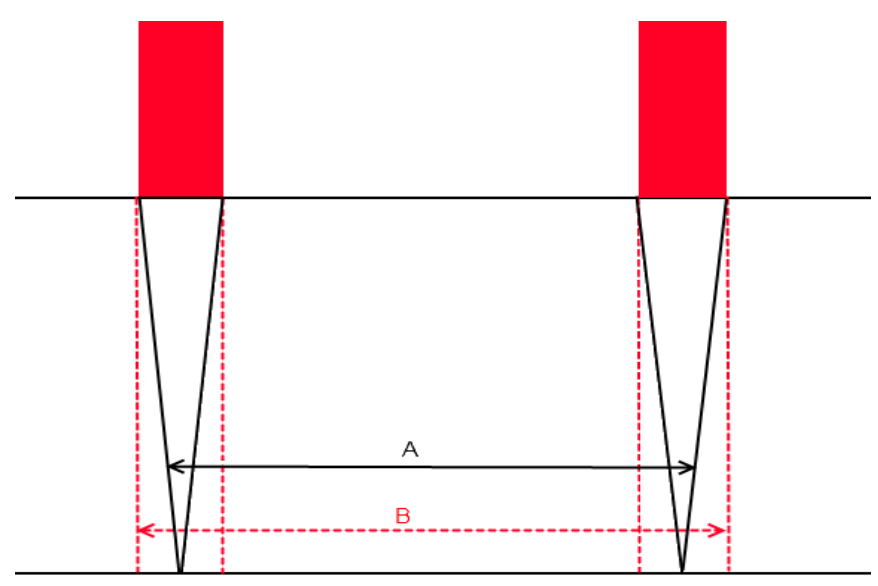

Figure 33 - Attenuation of laser power as a function of depth. Red solid lines indicate lasers, horizontal black lines indicate PMMA top and bottom. The dashed red lines and corresponding OD "B" are the theoretical laser output. However, as depth increases, laser penetration decreases to create a shrinking OD "A"

The most effective design involved a downward facing channel with through-holes slightly smaller than the tygon tubing OD. The downward facing channel allowed for a wide tolerance for tubing insertion depths. Using a pair of fine tweezers the tygon tubing was squeezed and inserted into the through-holes where it was allowed to expand and create a loose seal against the PMMA walls. Adhesive was then applied around the surface of the chip to strengthen the seal. This chip proved most successful and was used for all subsequent chip construction.

\subsubsection{Electrode Design}

One of the more difficult parts of creating a low-cost microfluidic chip is the implementation of functioning electrodes. Commonly, sub-micron thick layers of conductive metals are patterned onto glass slides to create functioning electrodes by either positive resist lithography or sputtering that require expensive equipment. ${ }^{43}$ Keeping with the low-cost theme of this microfluidic experimentation, cheaper and more accessible 
methods were used. The following designs were based off literature, other ideas within the microfluidic research group and industrial design.

The high voltage source used during experimentation readily accepted 20 -gauge wire so most chip construction ideas involved this wire. Using a pair of wire cutters two lengths of wire were cut so the ends formed a sharp point. These points were pierced through the tape on a tape-backed chip and sealed in place with adhesive. Although the wires raised the chip height from the microscope stage the microscope was still able to focus through the increased distance and into the channel.

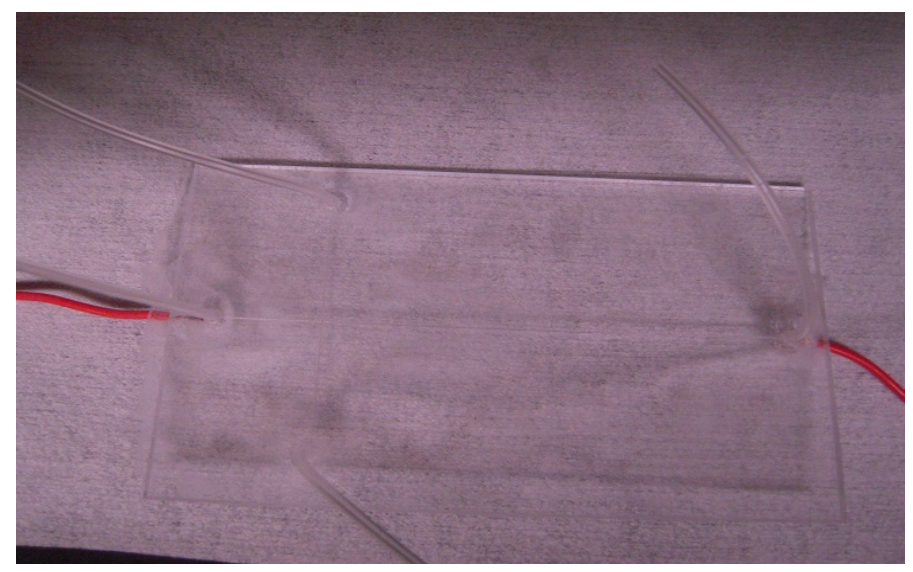

Figure 34 - 20-gauge electrode wire pierced through the tape backing and sealed with loctite adhesive

Due to the structure of the tape, it would not allow simple punctures and would create a slit longer than the diameter of the wire. Attempts to seal the electrodes in place and make the chip watertight with adhesive were unsuccessful as the adhesive would continually seep into the channels.

Inspired by the thin electrode designs from literature ${ }^{43}$ aluminum foil was cut into the shapes shown in the following figure and sandwiched between the PMMA and the tape coating. 


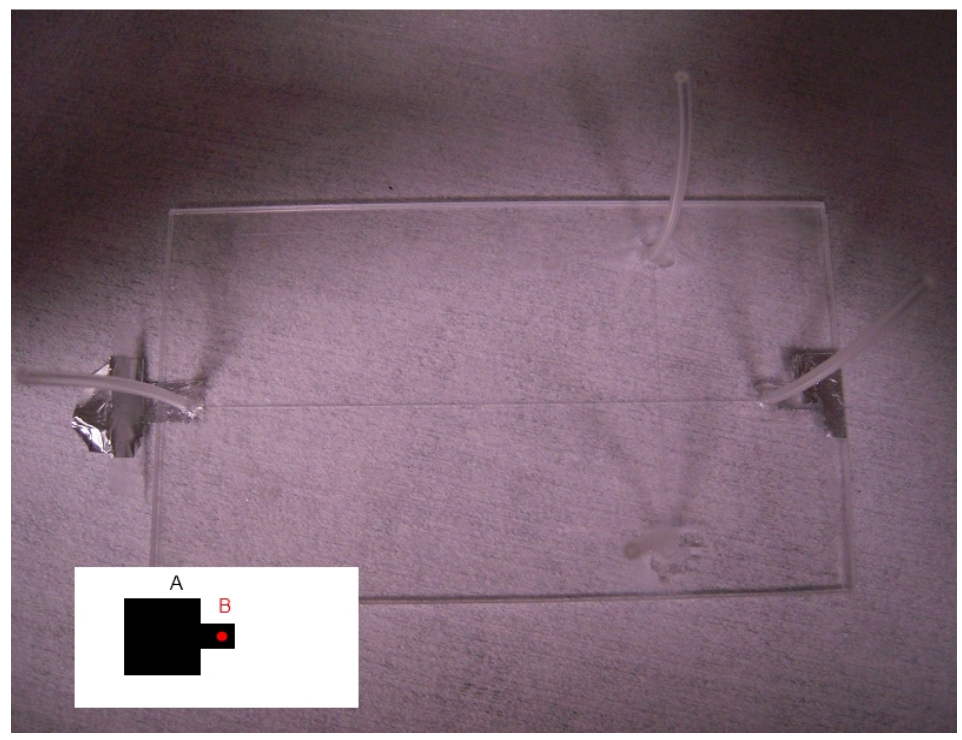

Figure 35 - Aluminum foil electrode design. The inset picture is a diagram of the foil electrode, A. B indicates the location of the tygon tubing hole with respect to the electrode

The electrodes had a large square area outside of the chip where alligator clips were attached. Between the tape and chip interface, the electrode area was a square that was slightly wider than the diameter of the through-hole that held the tygon tubing. The hope of this design was that the tension applied by the tape would create enough pressure to prevent an excessive amount of leakage between the foil and the PMMA. Some leakage still occurred and adhesive was applied at the edge of the chip between the electrode and the chip but leakage still occurred. This chip was successful in creating a voltage across the chip, but other methods were still pursued to find a better solution.

In an attempt to fix the leaking issue, another foil design was attempted . 


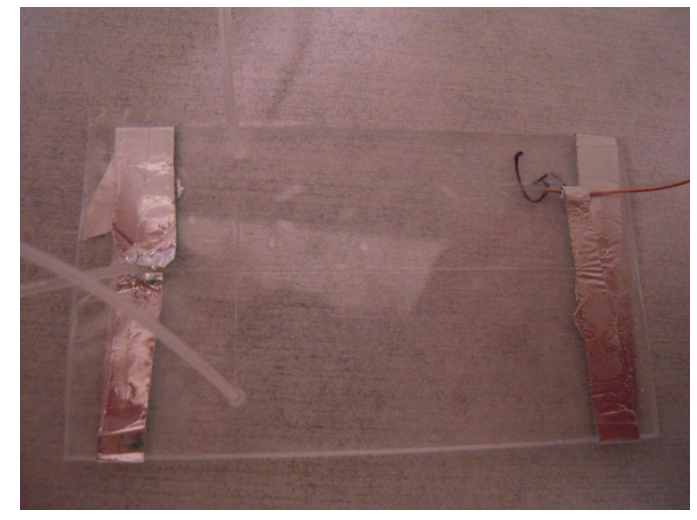

Figure 36 - Second electrode design involving aluminum foil. Foil is secured in place with $3 \mathrm{M}$ tape

A second design with aluminum foil electrodes was attempted to prevent the problem of leaking in the previous design. A strip of aluminum foil was wrapped around the chip and secured in place with the same tape used to seal the channel. Holes were cut with a razor blade to insert the tubing into the chip. This idea was equally successful at creating a potential down the length of the channel but was equally unsuccessful at preventing fluid leaks.

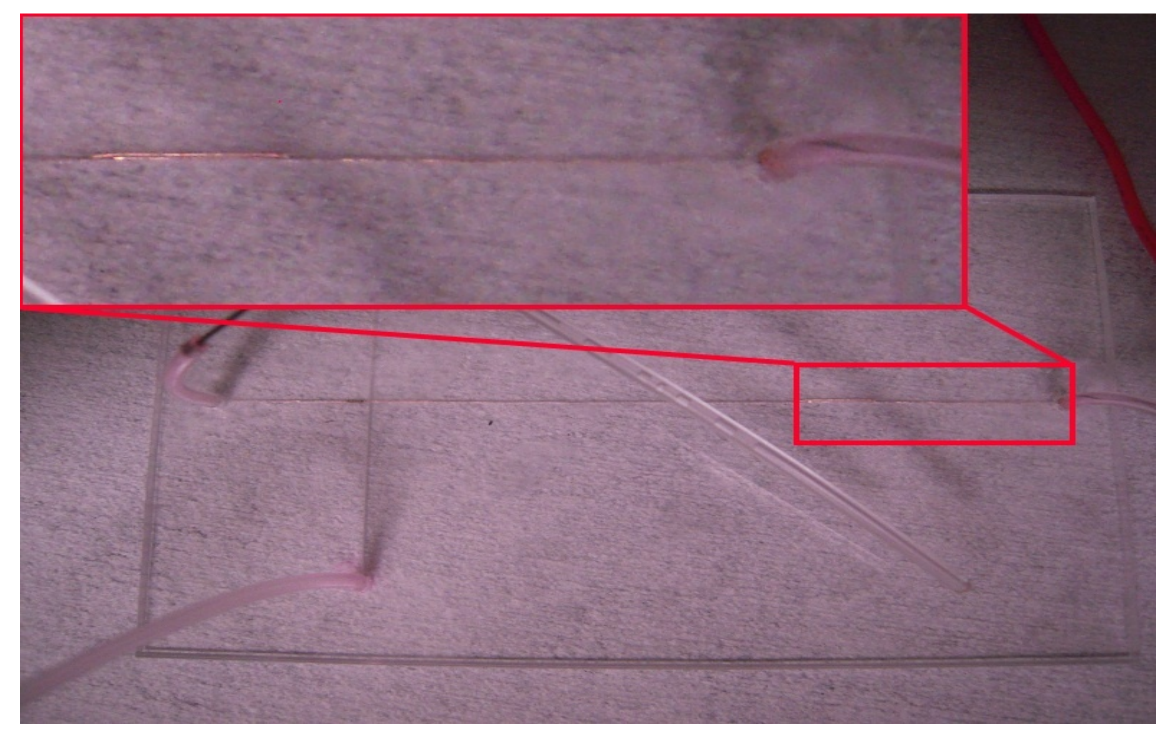

Figure 37 - Threaded electrode design with 40 gauge wire 
Electrode wire was threaded down the length of the channel, into the tygon tubing and squeezed in between the tygon tubing and the stainless steel hypodermic tubing before wrapping around a length of 20 gauge electrode wire as shown in the following figure.

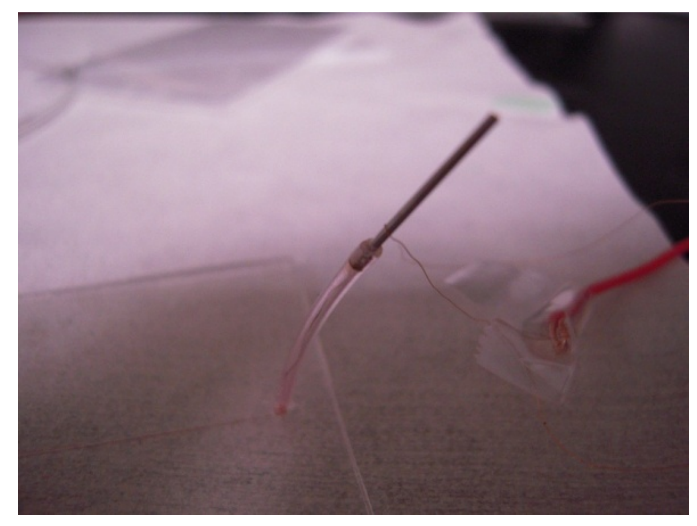

Figure 38 - 40 gauge wire threaded into tygon tubing and connected to 20 gauge insulated copper wire

This design was unsuccessful at inducing movement of charged microspheres. The idea was attempted again, except with the 40 gauge wire only threaded into the tygon tubing and not into the channel. The thought behind this design was a relatively large amount of surface area of the exposed wire would create a potential across the channel. This idea was unsuccessful as well as the resistance proved too high to warrant using this design.

Inspired by the success of other microfluidics group members at Cal Poly, the next idea involved threading 40 gauge wire through the reservoir hole in the chip. Other microfluidic researchers within the research group use simple sewing needles and 40 gauge copper wire to sew electrodes into PDMS chips. 


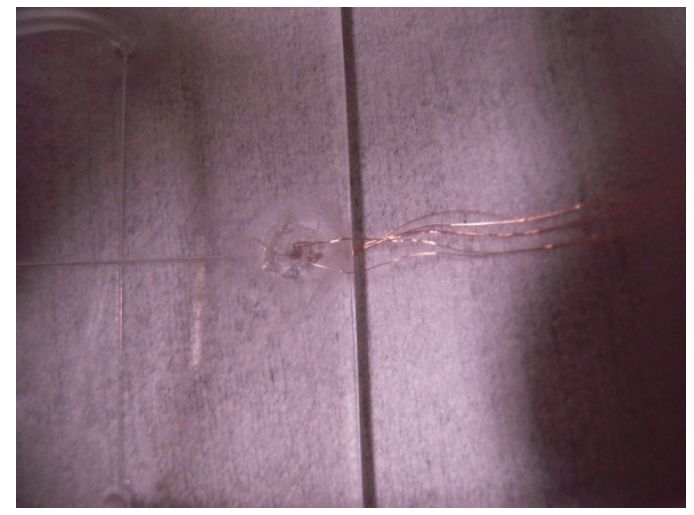

Figure 39 -Threaded electrode through chip reservoir

For this design, tape was applied to the chip to form a seal. Single loop passes were attempted first but electrodes broke too often to make this a viable option. The tape was pierced with an 18-gauge sewing needle and several passes were made to create several loops of electrodes which were spun and connected to a 20 gauge copper electrode wire. Tygon tubing was pinched and forced into the reservoir opening and sealed in place with adhesive. Adhesive was used to seal the pierced tape with the protruding copper wire. This method was successful at creating a current flow through the chip but exhibited high resistance. On some chips the small surface area of the wire was covered with adhesive to the point that the chip could not be used, creating a high fallout rate.

The most successful idea involved using the hypodermic needle connections between the tygon on the chip and the tygon connected to the syringes as electrodes. Stainless steel is not as good a conductor as copper but has increased corrosion resistance compared to copper. 20 gauge copper wire was wrapped tightly around the outside of the tubing as shown in the following figure and connected to the high voltage source. 


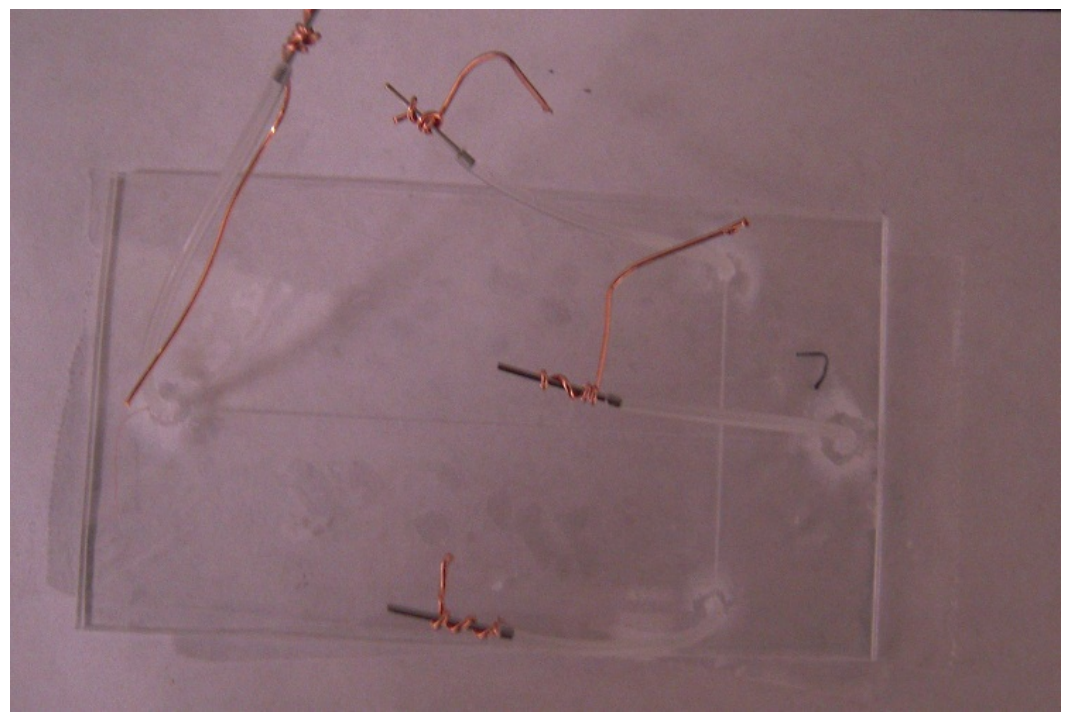

Figure 40 - Four electrode design with 20 gauge copper wire wrapped tightly around 23 gauge stainless steel hypodermic tubing

This idea ended up being the most successful and was used in final experimentation with a two-electrode design creating a potential down the length of the horizontal channel. In some CZE applications electric current is used to move a sample across the vertical cross-channel instead of pressure-driven flow. This electrode design can easily be adapted to said design. However, the four electrode design was not used in final experimentation. 


\subsubsection{Chip Bonding and Sealing}

There are several possible methods of bonding PMMA together to create enclosed microfluidic chips each with their individual strengths and drawbacks. The goals associated with bonding a chip are to create and interface that does not impede or interfere with experimentation. The different bonding methods were tested using a leak test involving a syringe with green food coloring. Green food coloring was injected into the reservoir on the chip and was pressed with a gloved finger to increase the pressure and force fluid through the channel. Observing the chip through a microscope against a white background it is very easy to see where chip sealing fails and where blockages are located.

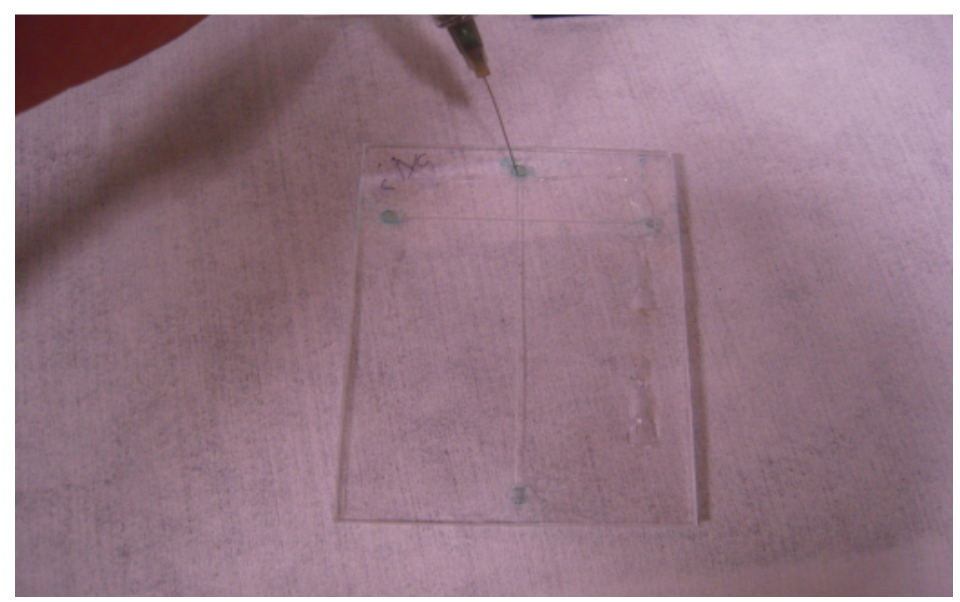

Figure 41 - Leak testing using a syringe loaded with green food coloring

\subsubsection{Chemical Process}

Bonding polymers with a strong solvent has been the standard method for many years outside of the realm of microfluidics as it creates a very strong bond using relatively little adhesive. Adhesives such as 2, 4 pentadione ${ }^{44}$ have been used to bond microfluidic chips. The drawback to this method is the possible clogging of microchannels by the bonding agent. Cholorocarbon solvents (chloromethane, dichloromethane, chloroform) are good for bonding PMMA, but for this application they work too well. Applying the precise 
amount of adhesive to seal the channel while not allowing any to leak into the channel proved too difficult to warrant moving ahead with this method.

\subsubsection{Microwave Processing}

Within the last year, a new low-cost method has come about that combines solvent, thermal and microwave bonding while at the same time using cheap and ubiquitous equipment. The method involves a weak solvent, a microwave radiation source and office binder clips. The weak solvent is an alcohol, the most effective in this case being ethanol.

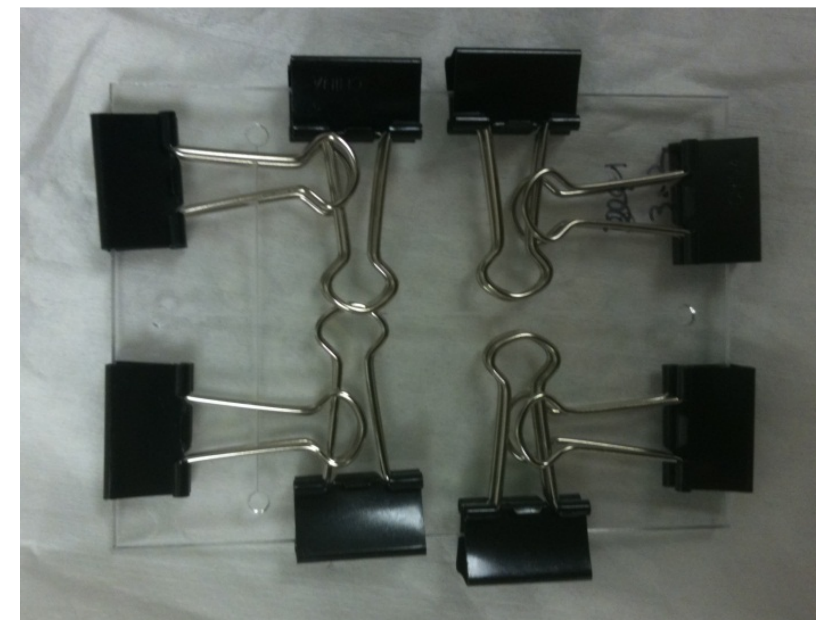

Figure 41 - Initial setup for microwave bonding, with $3 / 4$ " binder clips to focus the heat

The binder clips, as shown in the experimental setup below, act as collectors and concentrators of the microwave radiation. This effect has already been patented and is used in many food packaging applications. The metal is thin enough to absorb radiation and generate eddy currents that heat the metal. Additionally, when the clips reach their curie point, the radiation they absorb drops off significantly so they will maintain a somewhat constant temperature. The clips also act to apply pressure to the edges of the chips to increase bonding strength similarly to the method described in the thermal bonding section. 


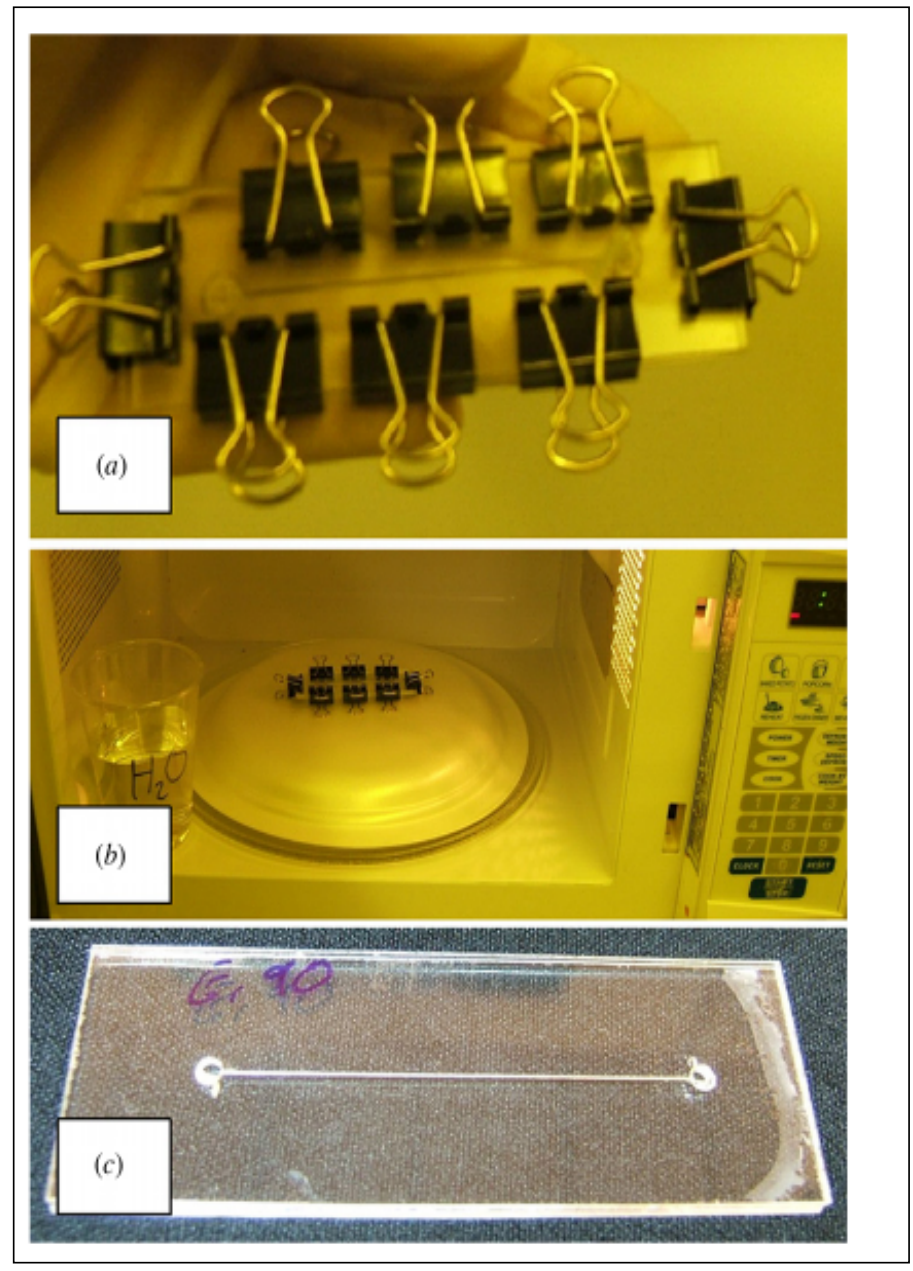

Figure 42 - Experimental bonding setup used by Rahbar et al employing organic solvents and microwave radiation $^{37}$

The advantages of this method are the ubiquity of the materials and equipment required as well as the precision of the technique. Chip cooling and heating in thermal bonding have much longer ramp-up and ramp-down times compared to the microwave and are more sensitive to fluctuations in temperature in opening the oven door and inserting the sample. It is also advantageous over pure solvent bonding, as the difference between a properly bonded chip and one with solvent obstructing a channel is on the range of microliters. Optimization of manufacturing with this technique is also an easy task as the experimental setup is easy to standardize between tests and. 
Rahbar et al noticed that different alcohols were able to bond the PMMA to different levels as is shown in the following graph.

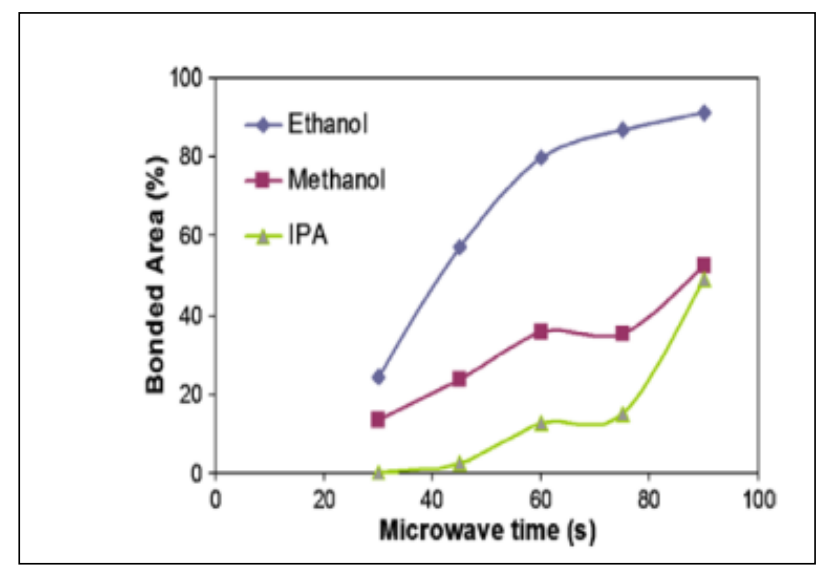

Figure 43 - Percentage of bonded area as a function of microwave time and solvent ${ }^{37}$

Ethanol was far and above the best solvent to use so it was chosen for this experimentation. For the experiment, ethanol was applied to the entire surface of the PMMA slide containing the channels using a syringe. The blank PMMA cover was placed on top and pressure was applied until the ethanol had completely filled the interface between the two pieces. The 60second data point was chosen as a starting point for experimentation. When observing the first trial the experiment was stopped after 30 seconds after the chip began to break down thermally at an increasing rate.

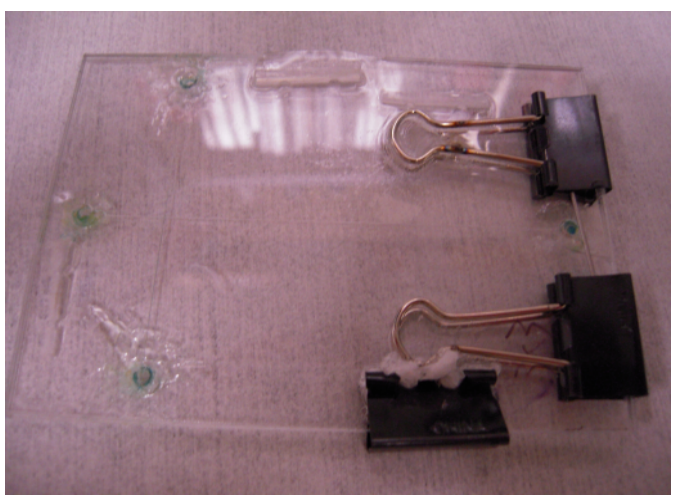

Figure 44 - Initial trial of microwave-based chip bonding. The binder clips that are still attached became irreversibly bonded to the PMMA after 30 seconds 
Subsequent trials were attempted with varying lengths of time and power.

As mentioned in literature, the rapid bonding process used in microwave/ethanol bonding leads to damage to the chips at the interface where the ethanol quickly evaporated, as shown in the following figures.

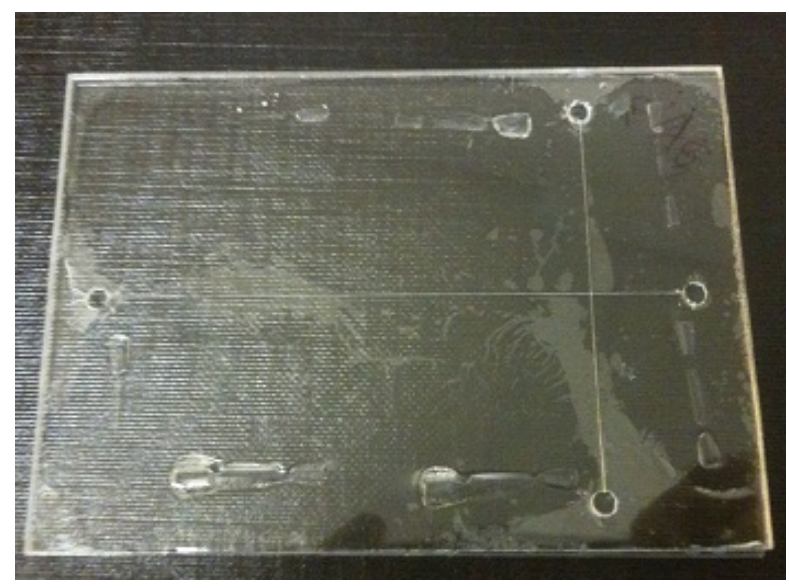

Figure 45 - Macroscopic view of chip damage during microwave bonding. Around the edges is the damage caused by the binder clips and around the channels is the damage from the quickly evaporating ethanol

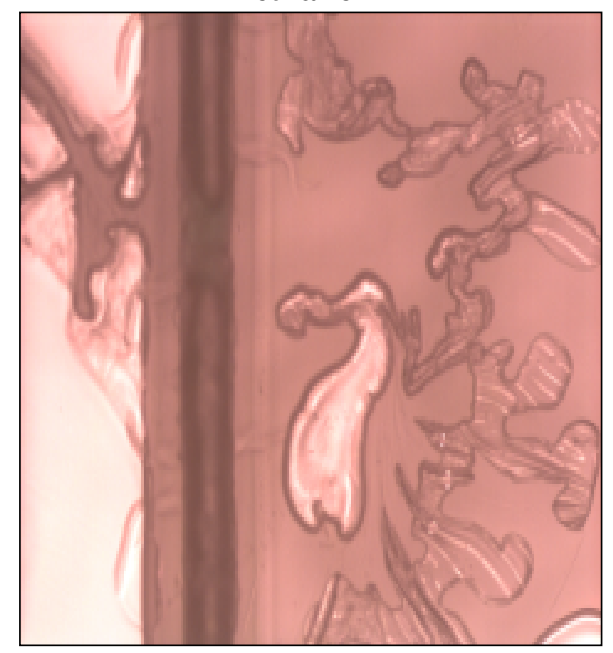

Figure 46 - Radiating patterns of damage in PMMA chip due to microwave bonding, microscopic view. Channel width is approximately 240 microns wide

In addition to the visual contamination this causes, there are also detrimental effects to the function of the chip in the form of blockages. The following chip was only microwaved for 
two periods of 10 seconds, far short of the time used by the researchers in literature yet it created several blockages along the channels.

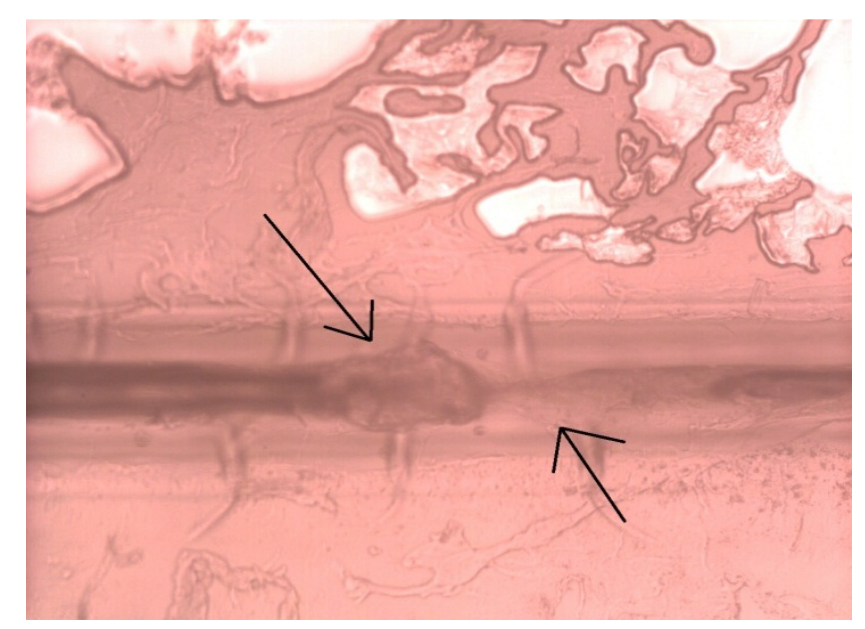

Figure 47 - Microscopic view of microwave bonded chip. Channel width is approximately 240 microns. Note the region on the left where the channel has become obstructed by a blockage and the region on the right where the channel has collapsed.

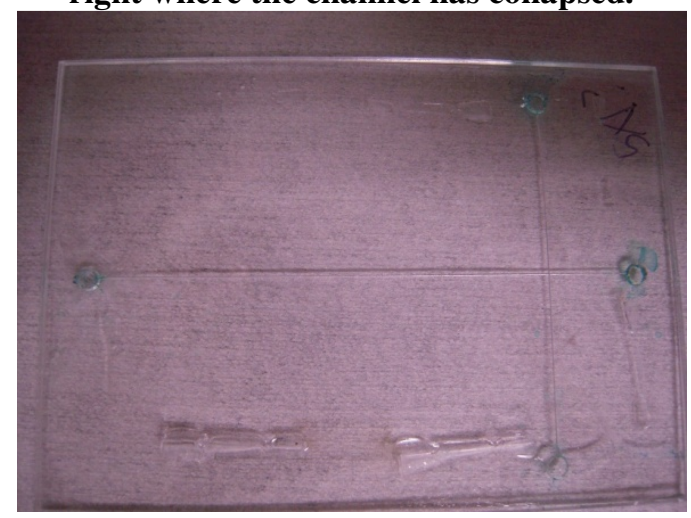

Figure 48 - Leak testing of microwave bonded chip. Dye was not able to travel down the channels further than a few millimeters without hitting an obstruction

Several other trials were attempted with the ethanol and microwave method of bonding, but finding the span of time and power settings that created a strong bond yet did not collapse the channels was not found. Due to the destructive nature of this bonding method, meaning that chips could not be reused from either failure case, this method was not pursued further. 


\subsubsection{Thermal Process}

Thermal bonding of PMMA depends highly on the glass transition temperature of the material, or the range where the material transitions from being 'glassy' to 'rubbery', where the molecules of the polymer in this case will become more excited. Depending on its manufacturing process and makeup, the glass transition temperature covers a fairly wide range, from 85 to $165 \mathrm{C}^{45}$, but most will fall in the range of about 100-120 C. However, because PMMA is an amorphous solid the width of the glass transition temperature allows a certain amount of room between the glass state and the liquid state. For crystalline materials, the glass transition temperature is a much smaller range.

When bonding PMMA with microstructures using a thermal bonding process, a lower temperature is desired. Microstructures such as posts and thin walls formed in polymers that have a higher surface area to volume ratio than the rest of the chip will experience higher thermal absorption and possibly lose their shape. Some microfabrication processes are dependent on this effect, particularly in the study of microlenses.

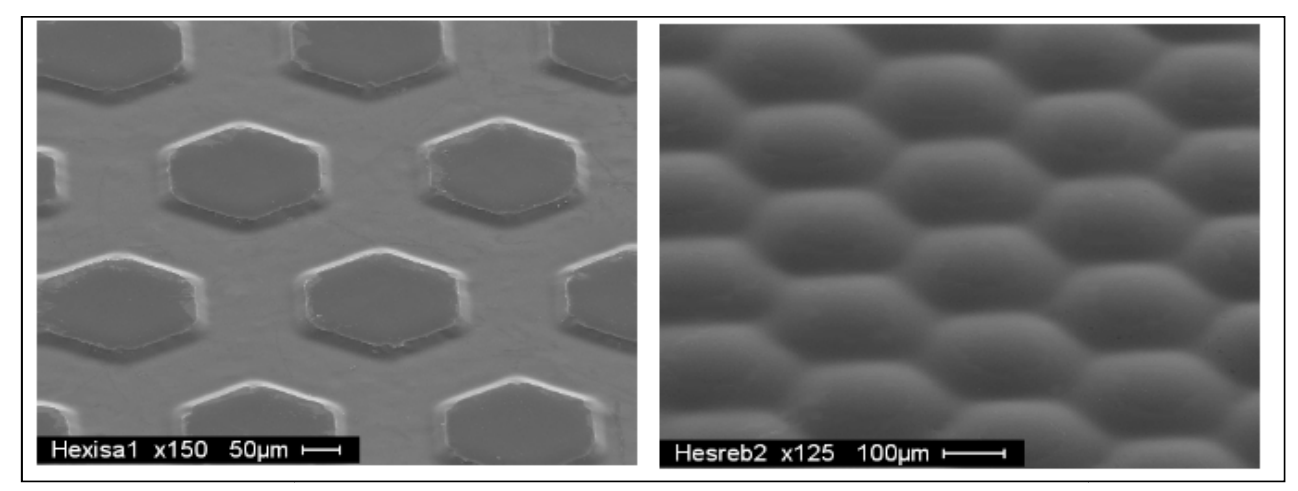

Figure 49 - Hexagonal polyethylene terephthalate microlenses before (left) and after (right) thermal annealing at $160 \mathrm{C}$ for between $30 \mathrm{~s}$ and 3 mins ${ }^{46}$

In this study, hexagonal microstructures were patterned onto a silicon substrate using a photolithographic process. After the fabrication process, the lenses underwent a thermal annealing process at $160 \mathrm{C}$ for times between $30 \mathrm{~s}$ and 3 mins, above the glass transition 
temperature of PET, $70 \mathrm{C}^{45}$ However, because these microstructures were not complex and rose only a few micrometers above the surface, they required a fair amount of heat before reflowing into the picture on the right. Using a thermal bonding process is a somewhat tricky process then, as the process must follow strict guidelines based on the makeup of the polymer to reach the intended end result without going too far.

Zhu et. al. put forward a method for thermally bonding microfluidic chips made from PMMA. ${ }^{47}$ They found that using an optimized bonding temperature of $95^{\circ} \mathrm{C}$ with surfacemodified chips, external pressure, and post-bonding annealing gave the best results. For the purpose of this thesis, however, we will be using minimally processed chips but will use the parameters from the rest of the processes. The surface-modified chips involved using MMA monomers at the interface between the two layers of chips.

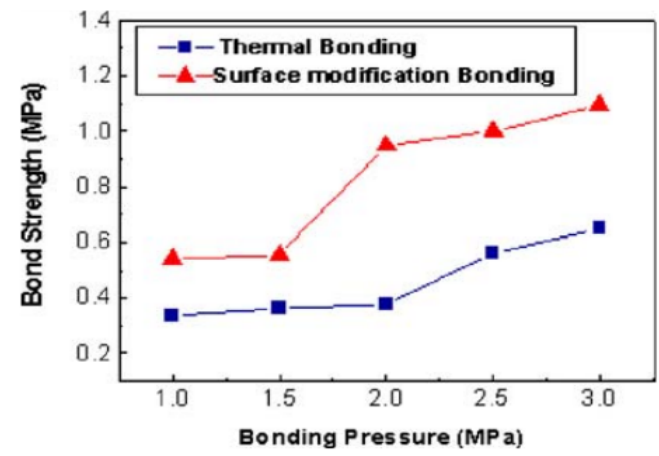

Figure 50 - Bonding pressure's effect on bond strength for bond of $95^{\circ} \mathrm{C}$ and 3 minutes ${ }^{47}$

For the untreated chip, there is a fair increase in bonding strength above the $2 \mathrm{MPa}$ bonding pressure. For this project, we will be shooting for between 2.5 and 3.0 MPa bonding pressure. For both the bond pressure test and the following bond temperature test, the chips were bonded for 3 minutes. 


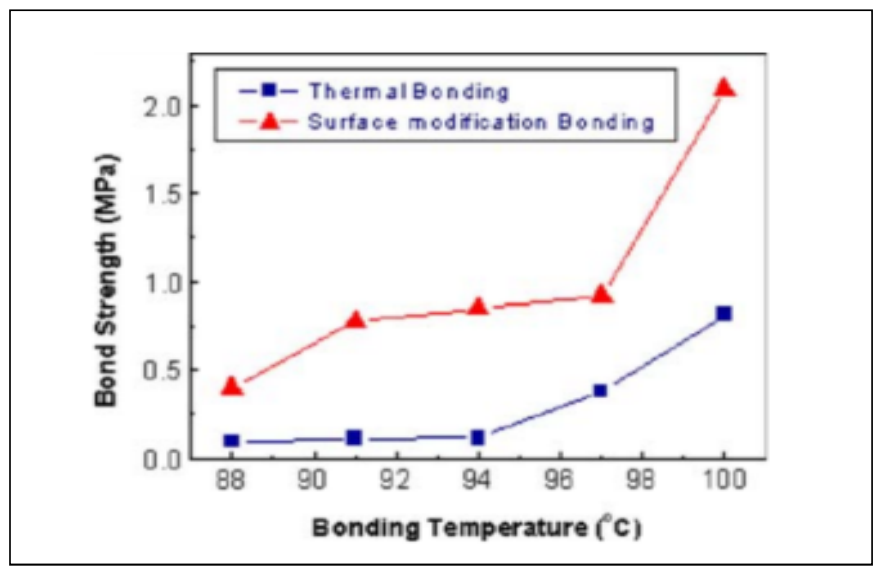

Figure 51 - Temperature's effect on bond strength. Bonding pressure and time are 2MPa and 3 minutes 47

Bond strength is also heavily dependent on the temperature used. The oven used for this process must therefore be accurate and be able to keep a small temperature scope for the duration of the bonding. A few degrees below the optimal bonding temperature and the bond strength drops by almost an order of magnitude (97C to 94C). At 97C, a few degrees above the optimal 95C maximum temperature Zhu et al used, the walls of the microchannel began to drape. This thesis is using laser-etched channels with a smoother profile than the 90 degree squared channels used in the thermal annealing study, so there will be a slightly wider temperature range between optimal bonding temperature and the wall drooping temperature. 

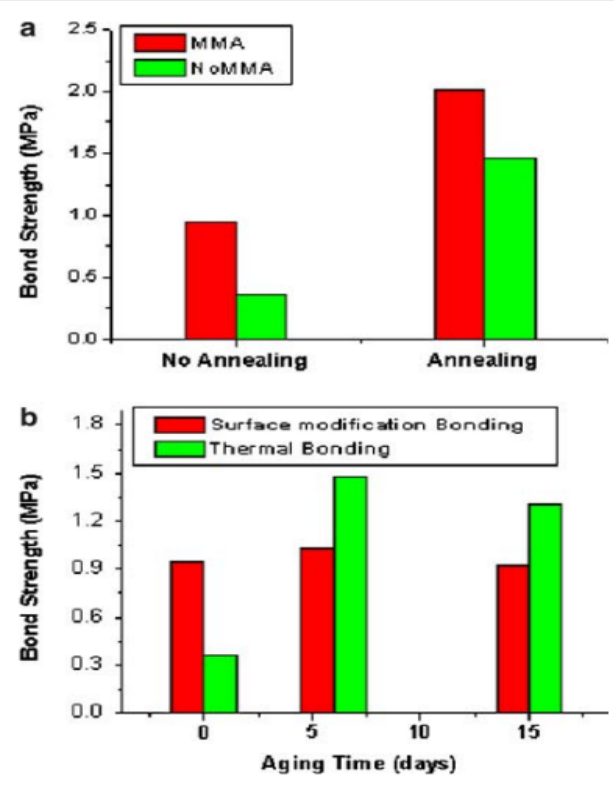

Figure 52 - Aging's effect on bond strength. Bonds were created with temperatures, pressure and times of $95^{\circ} \mathrm{C}, 2 \mathrm{MPa}$ and 3 minutes ${ }^{47}$

After the initial bonding process, an annealing process was used to strengthen the bond. The annealing process involved holding the chips at a temperature of $50 \mathrm{C}$ for 2 hours. From the data, the annealing process roughly doubled the bond strength. After the bonding and postprocessing stages, the chips were allowed to age for 5 and 15 days. While there wasn't a significant effect on the surface-modified chips, the unmodified chips experienced nearly a 5 -fold increase in bond strength after 5 days.

Several trials were performed using a small laboratory oven. A total of three trials were performed to bond the chips using only thermal energy. All three trials consisted of sets of three chips that were held together with the same binder clips used for the microwavebased bonding and held within the range of $95-97^{\circ} \mathrm{C}$ for at least $90 \%$ of the 2 minute mark reported by literature ${ }^{47}$. 


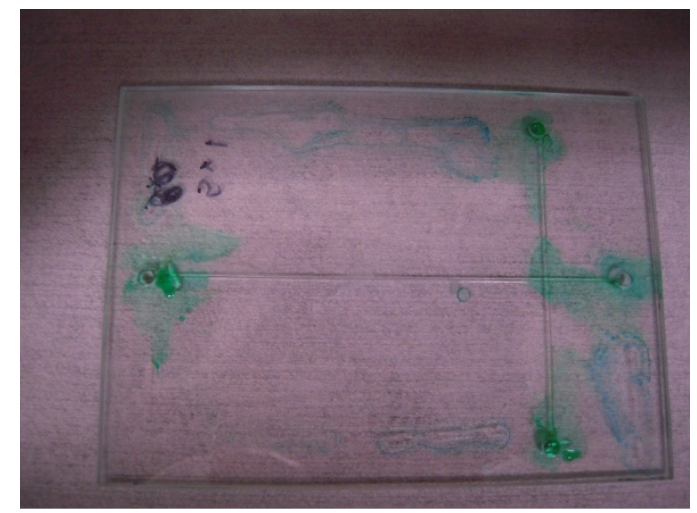

Figure 53 - Leak testing of thermally bonded chip and subsequent failure

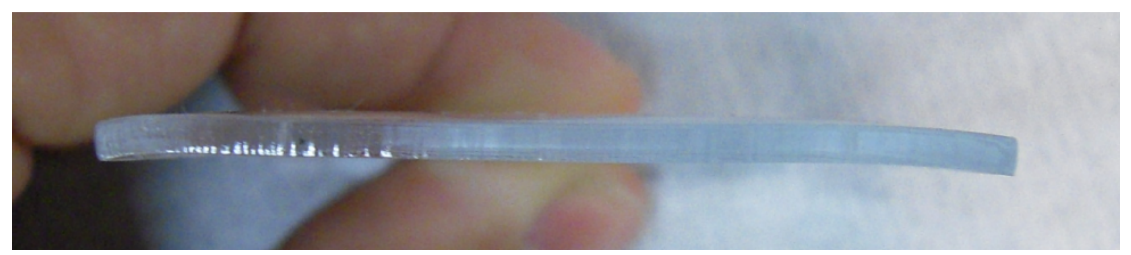

Figure 54 - Warped chip due to extended time at elevated temperature. It is difficult to show features on something that is optically clear but the edges of this chip are particularly warped

One of each of the chips was tested for leakage and all chips failed. One set was then held at an elevated temperature of $105-110^{\circ} \mathrm{C}$ for a time of 20 minutes to put the chips higher in the glass transition temperature zone than at the previous temperature. Instead of bonding, the chips ended up warping to the point that they became useless for experimentation.

Another set of chips were held at the annealing temperature of $50^{\circ} \mathrm{C}$ for 2 hours, a process reported to be successful at repairing cracks and voids in literature ${ }^{47}$. This method was unsuccessful at either bonding the chips or repairing the damage caused by laser ablation. From the failures in the initial trials, this bonding method was not pursued further.

\subsubsection{Tape-Based Backing}

3M 311 packaging tape is a polypropylene film-based tape with a pressure-sensitive acrylic adhesive commonly used to seal shipping boxes and measures .052mm thick. [data sheet] For this microfluidics application, the clear packing film works as an effective cover for the 
microfluidic channels, able to create a leak-proof seal while remaining optically clear. An additional trait of the tape is that it can be removed and replaced if the film is incorrectly applied or the chip is to be reused. This makes it incredibly advantageous for the low-cost theme in this thesis. PMMA is a much tougher material than cured PDMS and can be scrubbed with a scouring instrument between trials so long as the user has not performed any sensitive post-treatment methods on the chips such as methylcellulose application. Because this project uses PMMA as a sacrificial material for the microfluidic channels, there is very nearly zero risk of disturbing or destroying chip architecture with this backing method whereas with PDMS the user runs the risk of tearing posts and thin walls when applying or removing the tape. Additionally, the ejected material on the edges of the channels that forms a lip creates and effective seal for the PMMA as discussed in the laser ablation section.

3M 305 packaging tape has a polypropylene body with an acrylic adhesive attached. While this tape is optically clear to the naked eye, under the $10 \mathrm{X}$ magnification used for the experiments performed, the microstructure of the tape becomes more visible and the imperfections in the film become very apparent when experimentation starts. The film is 22.86 microns thick according to 3M's spec sheet. The LabSmith microscope can focus through $8 \mathrm{~mm}$ of material with a resolution of 1 micron, so this is not a difficult task to focus at the depth where most of spheres are moving in the channel. The tape is also wide enough to cover the length and width of the microchannels with one strip. The tape was applied on one end of the chip and pressed onto the back of the chip firmly to form a proper seal with the channel. This method formed a solid seal nearly every time. 


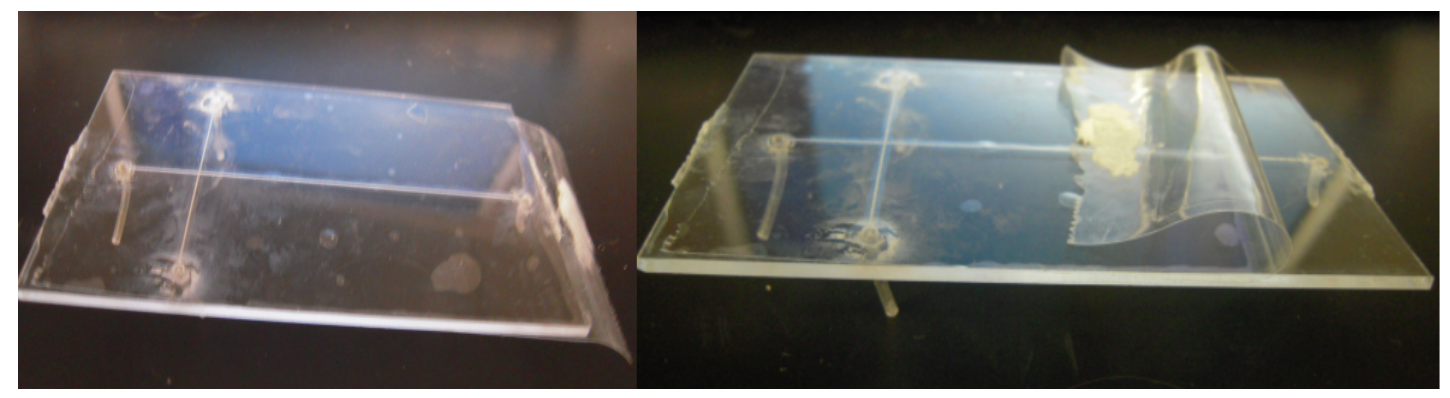

Figure 55 - Application of polypropylene packing tape onto back of chip

The difficulties arise when the imperfections in the tape are out of focus and contribute to a blurry artifact on the screen as shown below.

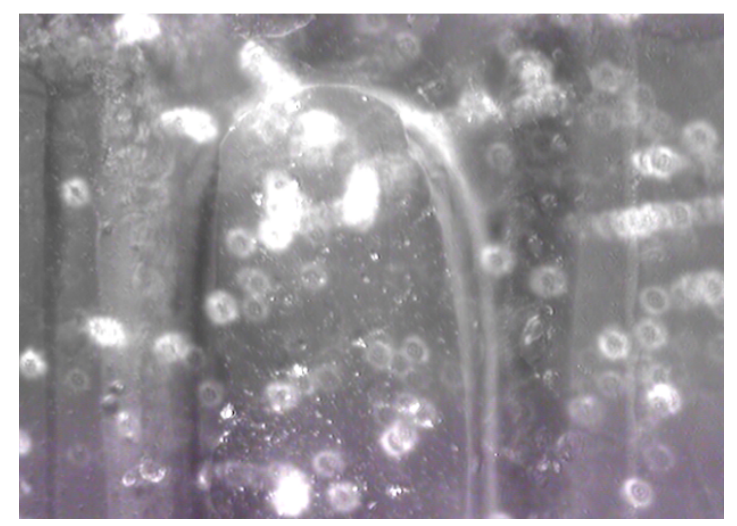

Figure 56 - Visual artifacts of out-of-focus imperfections in polypropylene microstructure

This becomes especially difficult when introducing a light source to the chip. Because the light source is very bright when comparing intensity vs. area for ambient light, the LED light source on the microscope can highlight imperfections to the point that they can make fluorescent particles very difficult to see past the out-of-focus microstructure. Using the polypropylene film is a good design choice for a cheap backing for a chip that only requires 4x magnification. Beyond that, the film becomes difficult to use for precise visual assessment. 
The $3 \mathrm{M}$ transparent tape is very optically clear, especially at the microscopic level. This is a huge advantage over the polyester tape for visual assessment of flow, especially for this experimentation tracking the movement of microspheres. The use of this tape had a small disadvantage in that it was only 1.0 " wide so multiple layers were required to cover the chip. At the junctions between tape pieces there was a small leak. This could be remedied by requesting the same tape in a wider format from a supplier. Application of this tape on a chip is shown below. The same method used with the polypropylene tape of firmly applying the tape down the channel

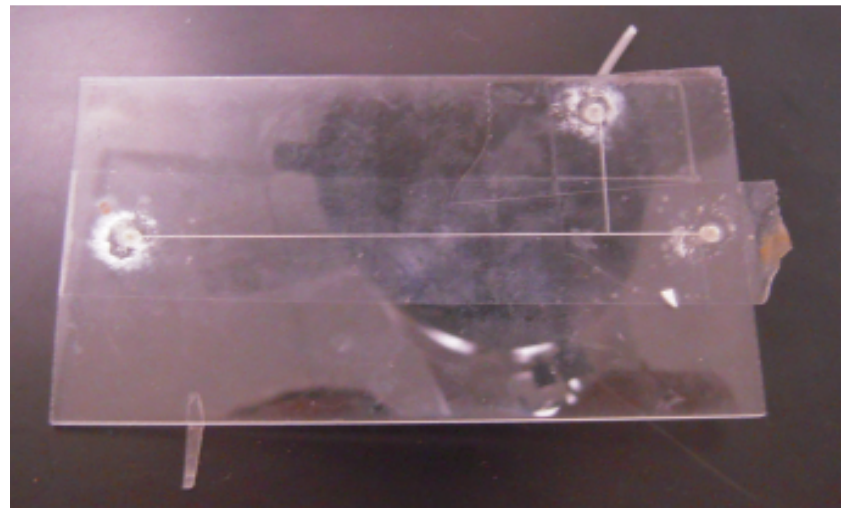

Figure 57 - Transparent tape used to seal the channels on a PMMA chip

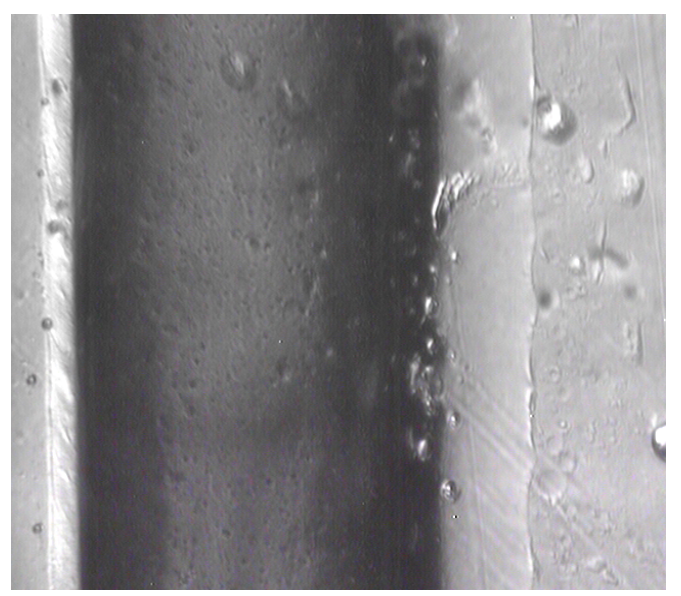

Figure 58 - 10x view of 3M Transparent tape backing on PMMA microfluidic chip 
The most distinct disadvantage of this tape during this experimentation was the fluorescent nature of the tape. When performing experiments with fluorescent microspheres there are difficulties as the tape itself is fluorescent, as shown in the following picture.

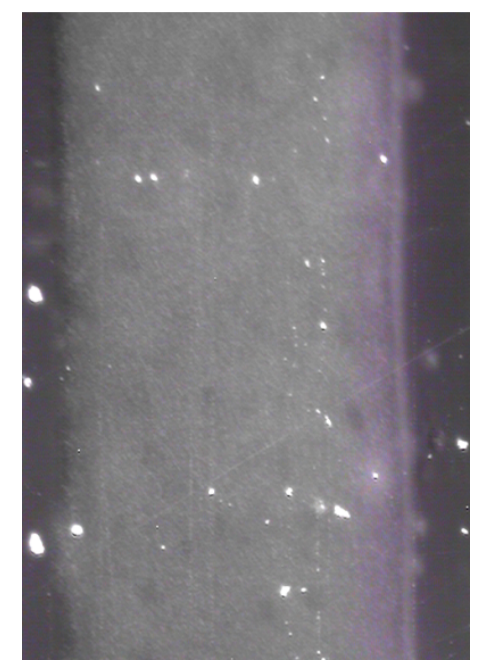

Figure 59-3M Transparent tape backing exposed to red light wavelengths and covered with the SVM chip cover that protects the chip from room lighting at $10 \mathrm{X}$ magnification

This makes it extremely difficult to observe fluorescent particles and proteins. Below is a picture showing fluorescent microspheres underneath the transparent tape. It is difficult to view these particles when they are in motion and they are nearly impossible to show in a picture 


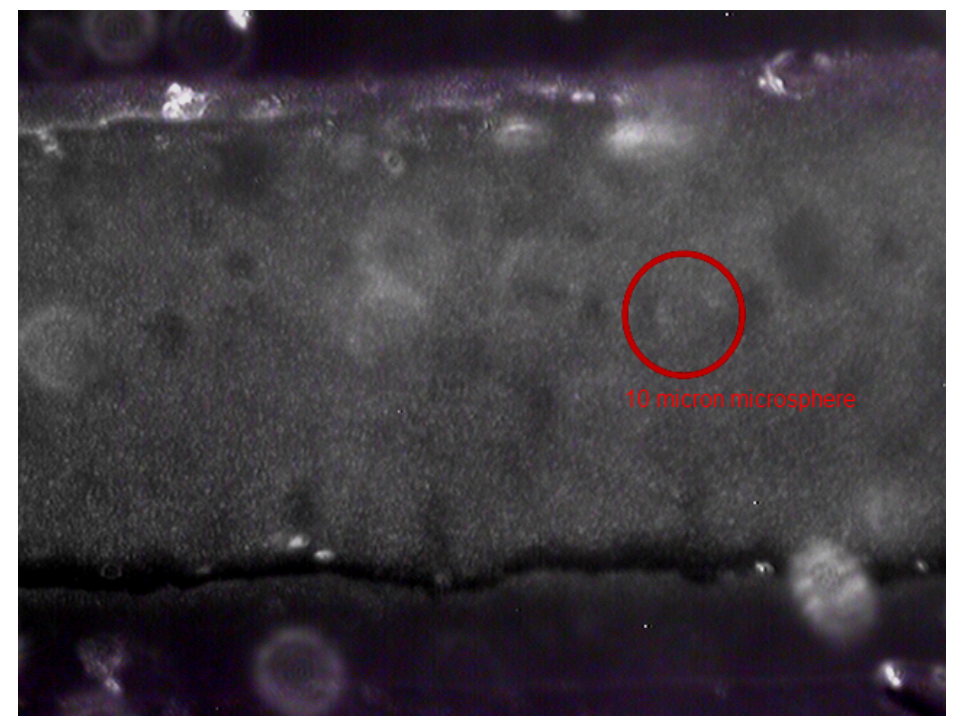

Figure 60- Microsphere shown beneath fluorescent tape. Contrast and brightness levels have been adjusted to allow for easier viewing and a 10 micron particle has been circled. $10 \mathrm{X}$ magnification 


\section{Experimental Design}

\subsection{Profilometer}

To measure the geometry of the laser-etched channels, a stylus profilometer was used. A profilometer is a device used to measure extremely fine geometries on the sub-micron scale. The device consists of a cantilever and a micromachined tip that traces along the surface of a sample. A laser shines on the cantilever and the horizontal displacement of the cantilever manifests as a change in the location of the incident laser spot. This displacement is then recorded and tied to the horizontal displacement of the cantilever. The data points can be plotted to show the geometry of a sample.

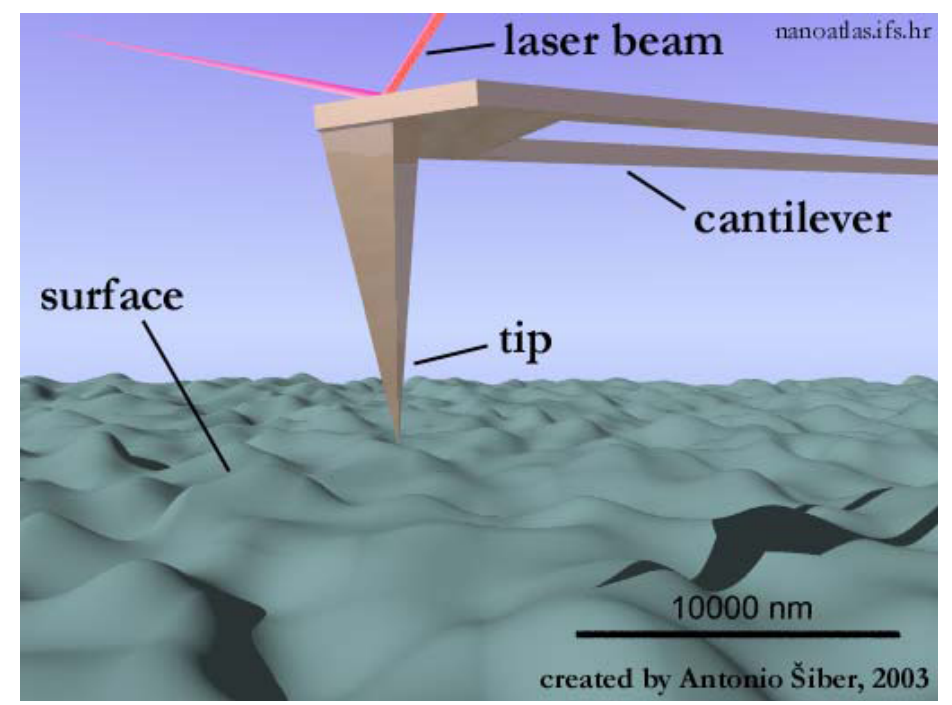

Figure 61- Computer model of a profilometer tip. Notice the laser beam reflecting off the top of the cantilever ${ }^{48}$

The profilometer used in this study was an Ambios XP-1 Stylus Profilometer, similar to the one shown in the following figure. 


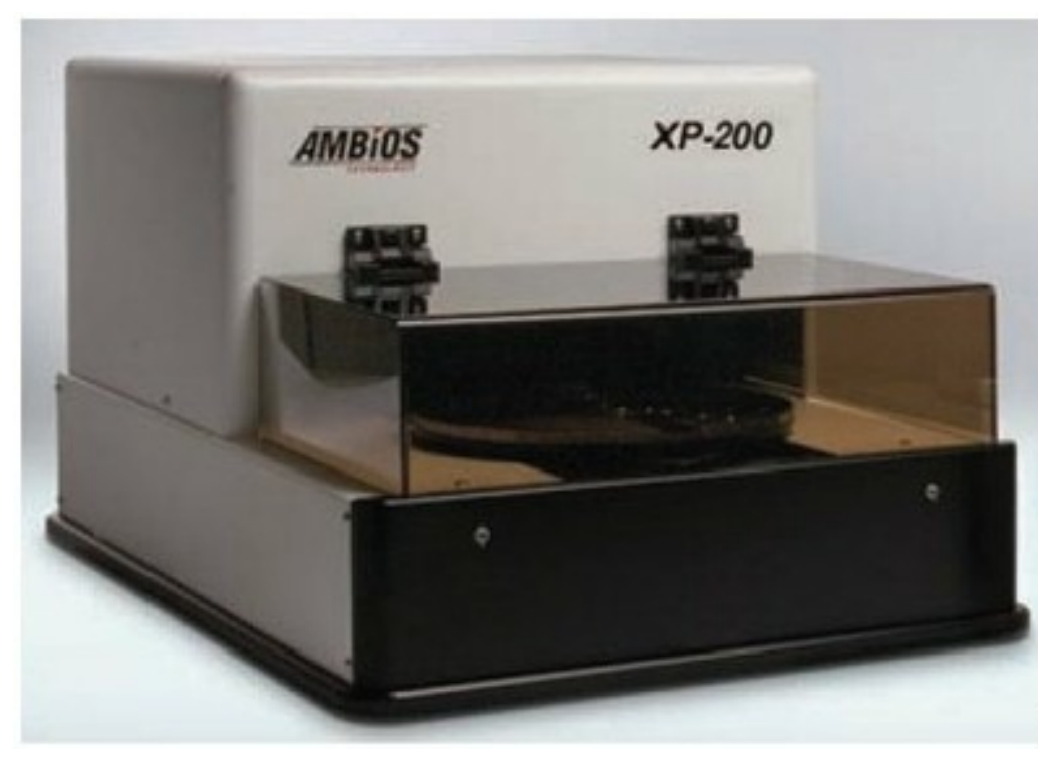

Figure 62 - Ambios XP-1 Stylus Profilometer ${ }^{49}$

\subsection{Equipment}

A LabSmith SVM340 microscope was used for imaging. This is an inverted microscope that contains both an interchangeable camera module and an interchangeable LED module. A black and white camera module was used because it affords better quality (600 lines of resolution vs. 480 lines of resolution for the color camera) and with increasing contrast it was easier to see the smaller particles. The camera is able to capture at 30 frames per second and this fact was used to calculate the velocities in the results section. Under the 10x magnification, the display is equal to a $600 \mu \mathrm{m}$ x $600 \mu \mathrm{m}$ area. This magnification factor was used for the majority of pictures taken for this thesis.

A LabSmith HVS448 3000V voltage source was used to provide the voltage potential across the chip. This device is capable of providing 8 channels of a range of $3000 \mathrm{~V}$ to a maximum of $6 \mathrm{~mA}$. There are three ways to deliver this DC voltage from the two leads for each channel: $0 \mathrm{~V} /+3000 \mathrm{~V},-1500 \mathrm{~V} /+1500 \mathrm{~V}$ or $-3000 \mathrm{~V} / 0 \mathrm{~V}$. The potential provided 
can either be dependent on voltage or current. For this experiment, the $0 \mathrm{~V} /+3000 \mathrm{~V}$ setting with voltage dependence was used to attract the carboxylated microspheres.

Three different microspheres were used. 1 micron carboxylated fluorescent polystyrene microspheres from Invitrogen (Catalog $\# \mathrm{~F} 8819$, lot 28650W) were used in the lead up to experimentation to understand how particles would behave in the chip. These spheres were also used during experimentation and will be referred to as "1 micron spheres". These spheres absorb light at wavelengths of $535 \mathrm{~nm}$ and emit wavelengths at $575 \mathrm{~nm} .5 .5$ micron micron carboxylated fluorescent polystyrene microspheres from Spherotech (Catalog \# CFH-5052-2, Lot R01) were also used for experimentation and will be referred to as "5 micron spheres". These spheres absorb light at $460 \mathrm{~nm}$ and emit light at $485 \mathrm{~nm} .10 .14$ micron carboxylated fluorescent polystyrene microspheres from Spherotech (Catalog \# FC07F, inventory\# L080530A, Bangs Lot\# 8724) were used for experimentation and will be referred to as " 10 micron spheres". These spheres absorb light at $480 \mathrm{~nm}$ and emit light at 520nm.

\subsection{Solutions}

The microspheres used were a very stable species and thus to keep in line with the low-cost microfluidic system a simple solution of $1 \% \mathrm{NaCl} w / v$ solution was created using $\mathrm{NaCl}$ from Sigma Aldrich and $18 \mathrm{M} \Omega$ DI water. The $\mathrm{NaCl}$ was added because the fluid needed to carry an electric charge down the channel and a $1 \% \mathrm{NaCl}$ solution was sufficient to create a resistance down the channel of roughly 1-1.2 M 2 , equating to a resistance of $176 \mathrm{mS} / \mathrm{cm}$. Tween from Sigma Aldrich was added to the solution to create 
$.04 \% \mathrm{w} / \mathrm{v}$ mixtures, as this was shown by observation to prevent clumping in greater than $99 \%$ of cases after vortexing.

\subsection{Experimentation}

The following experimental setup was used with the following chip design.

The 23 gauge stainless steel tubing connecting the tygon tubing between the syringes and the chip were wrapped with 20 gauge copper tubing that was then connected to the LabSmith high voltage source to create an electrical connection between the fluid and the voltage source.

The chip design was of the "step variety" and backed using the optically transparent $3 \mathrm{M}$ tape with the overlapping design from the chip design section of the paper. The chip was secured in place using the same polypropylene packing tape used as a backing for some of the microfluidic chip designs.

Three Harvard Apparatus Single Syringe Pump 11s were used to inject the $1 \% \mathrm{NaCl}$ solutions and the sphere solutions into the chip. The injection channel was the "start" end of the long channel and the buffer solution was injected into the arms of the shorter channel. This way, the length of the channel used for observation could be completely cleared between trials. The end of the analysis channel led to a waste container with the end of the tubing level with the chip to minimize the effect of siphoning.

The electrode setup of the experiment required an uninterrupted flow of conductive fluid travelling from the electrode tubing, down the tubing, through the channel, up the other end of tubing and into the downstream electrode tubing in order to carry an electric 
current down the length of the channel. If the flow is interrupted then charge will begin to build up on the electrodes, as they are both connected to insulative tygon tubing at either end. If the charge buildup occurs at the electrode then it can arc and destroy the tubing it is connected to. If the charge is built up across an air bubble in the channel, then the solution will arc to the steel base on the microscope.

\subsection{Microfluidic Experimentation}

The syringe pumps were set to a syringe diameter of $4.78 \mathrm{~mm}$ per the manufacturer's instructions. The buffer solution was injected into the channel at a rate of $.0201 \mathrm{ml} / \mathrm{min}$ (per syringe) and the sphere solutions were injected into the chip at a rate of .0105 $\mathrm{ml} / \mathrm{min}$ to fill the lengths of the channels with conductive fluid. Before beginning experimentation, the channels were checked for air pockets that would impede or block the flow of the voltage through the channel. A $1000 \mathrm{~V}$ potential was applied to the channel and the amperage was checked. If the amperage was within normal bounds $( \pm 20$ $\mu \mathrm{A})$, the experiment was allowed to proceed. If the amperage was higher, the resistance of the channel was checked with a multimeter, the tubing and chip was checked for air pockets and fluid was again pumped through the channel to clear any blockages.

Once the chip was cleared of blockages and air bubbles, the microscope was moved to the proper segment of the channel for experimentation. A 37-second limit was set for the video capture based on the available memory on the computer. A series of voltages were applied to the channels in order to fill a single video's worth of each voltage for analysis. Before the trials were started, a video of the channel at $0 \mathrm{~V}$ was taken to determine a baseline velocity for the spheres to subtract from the electrophoretic velocities. 
Because the software required time to process and encode the video, the voltage was reduced to 0 between each trial in order to preserve the spheres in the channels. Thus, the voltage sequence would look like the following with the corresponding video captures: 0V (capture), 500V (capture), 0V, 1000V (capture), 0V, 1500V (capture), 0V, 2000V (capture). For the observation at the beginning of the channel, the channel had to be reloaded with spheres using the procedure mentioned previously after the $1000 \mathrm{~V}$ trial, as the electric field had cleared the channel of spheres completely. The channel also had to be reloaded after the $1500 \mathrm{~V}$ experiment at the middle of the channel.

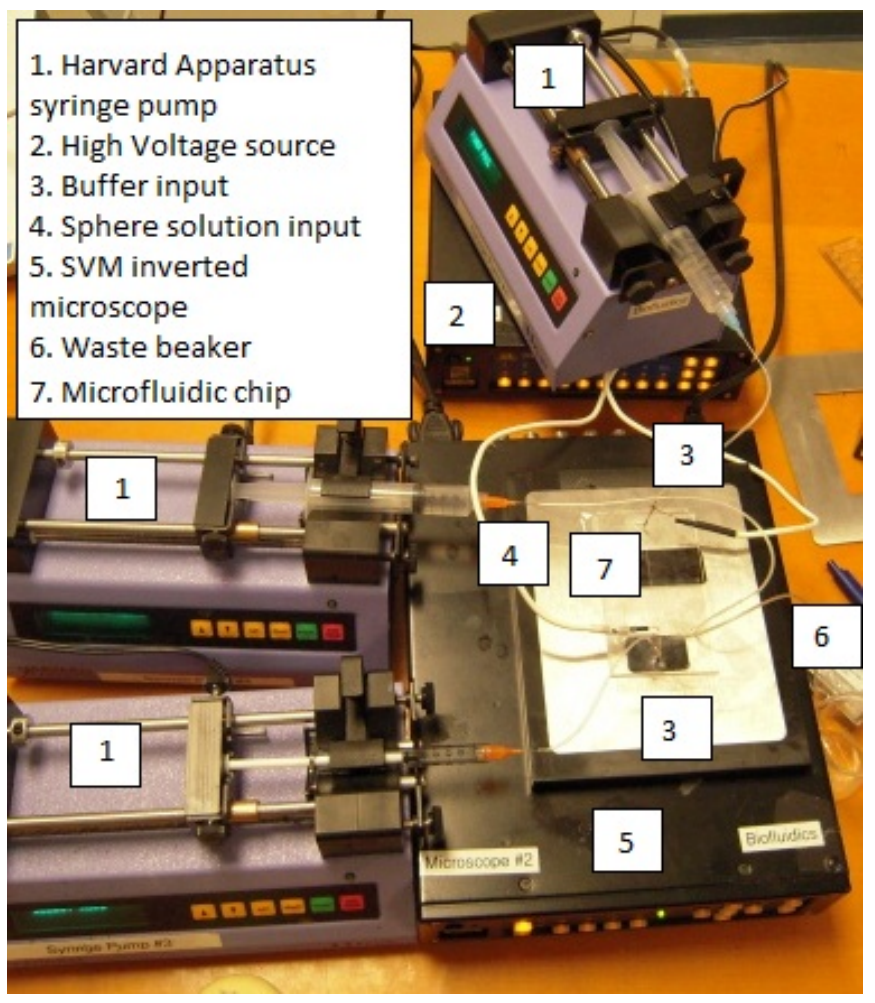

Figure 63 - Labeled experimental setup. Buffer input are at the top and bottom of the chip, sphere input snakes from left over top of chip and into right port. Waste port is on the left, tygon tubing snakes around into the waste beaker on the right. Harvard apparatus syringe pumps are used to pump fluid into the chip at a consistent rate. The chip lies on the stainless steel plate of the LabSmith inverted microscope. At the top of the picture, the syringe pump is sitting on top of the high voltage source. The white leads protruding from between the two machines are the leads from the high voltage source that attach to the ends of the long separation channel. 


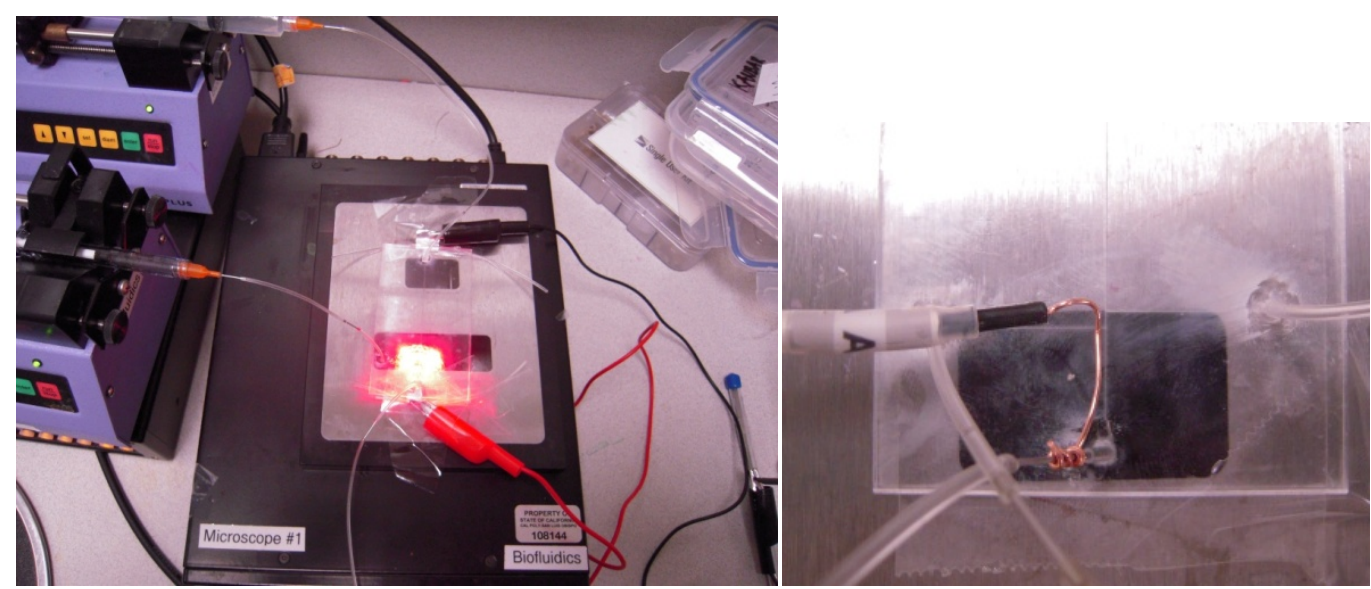

Figure 64 - Setup in use with SVM LEDs. Experimental foil electrodes are in use in this picture (L). Close-up of final electrode design in use ( $R$ )

\subsubsection{Software Modeling}

When manufacturing complex and expensive equipment like microfluidic chips, software modeling can cut down the time and cost of the project by a great amount. Microfluidic chips are often manufactured from PDMS and contain channels that have rectangular or square profiles. Laser etched channels, conversely, have parabolic profiled channels that elicit different flow rates. It is therefore beneficial to model experimental setups beforehand to reduce the chance of unforeseen events.

The purpose of software modeling for this thesis was to show there is no profound difference in pressure-driven flow or electroosmotic flow between a square-profiled channel and a parabolic profile one. If the profiles between the two different geometries are very different, then the models used for square-profile channel microfluidics will have to be edited specifically for laser-etched microfluidics. If the profiles are similar then the models from PDMS microfluidics can easily be transferred over for future work. 


\subsubsection{Electroosmotic Flow}

Electroosmotic flow was modeled using COMSOL with a wall potential of -..013V

according to literature. ${ }^{29}$ The computer used to model the following was only able to

mesh the models to a rough degree, explaining the large patches of different velocity next

to each other. First, the parabolic channel was modeled.

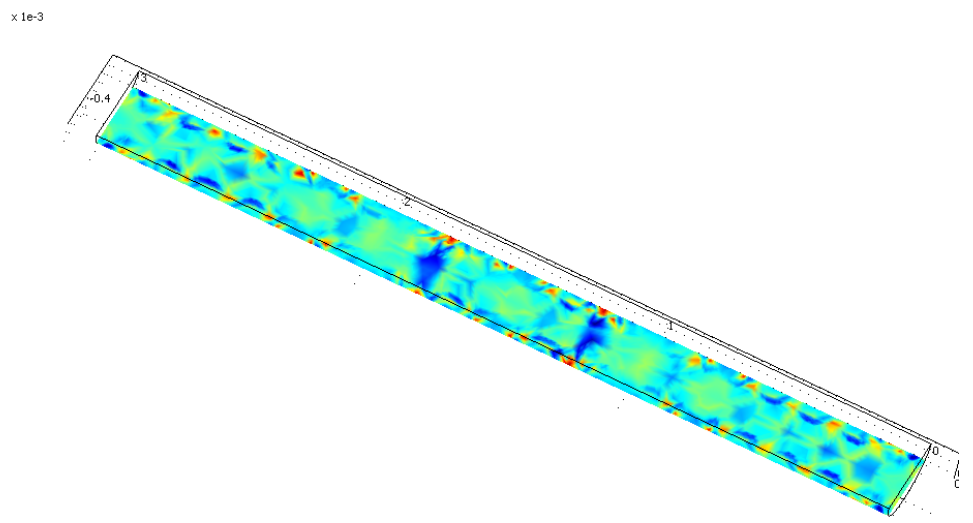

Figure 65 - Electroosmotic flow profile of a parabolic profile channel. Variances in velocity are only on the scale of roughly $0.4 \%$. The width of the channel is 350 microns, the depth is 120 micron and the length is $\mathbf{3 0 0 0}$ microns. This should be taken as an electrophoretic mobility graph, as increasing electric field strengths only scaled the small range of velocities by a scalar amount.

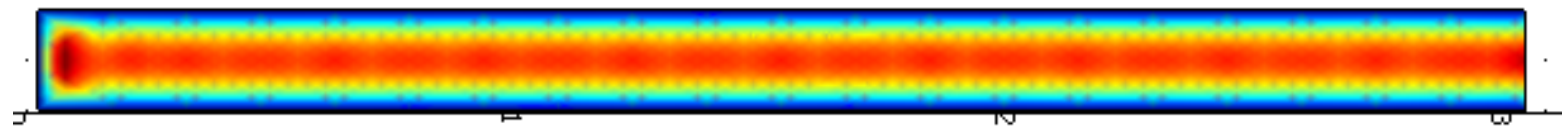

Figure 66 - Electroosmotic model of a square profile channel 120 microns by 120 microns wide and 3000 microns long. This should be taken as an electrophoretic mobility graph, as increasing electric field strengths only scaled the small range of velocities by a scalar amount. Velocities around the center are roughly the same and drop of steeply towards the edges.

Even though the same settings were used between the two models they netted very

different results. This is most likely due to the profiles of the two channels. 


\subsubsection{Square Profile Modeling}

The majority of capillary electrophoresis experiments use PDMS molds with square profiles. A square profile was the 'control' for the software modeling phase. A square profile channel was created with 200 micron long sides and a length of 1000 microns. The experimental conditions between the two profiles were kept constant - flow speed of $1 \mathrm{~mm} / \mathrm{s}$, conductivity of 200 Siemens/meter, temperature of $298 \mathrm{~K}$, no slip wall conditions and DC electrostatic potential of $10 \mathrm{~V} / \mathrm{mm}$. A fine mesh was created to create a more accurate output and the following resulted.

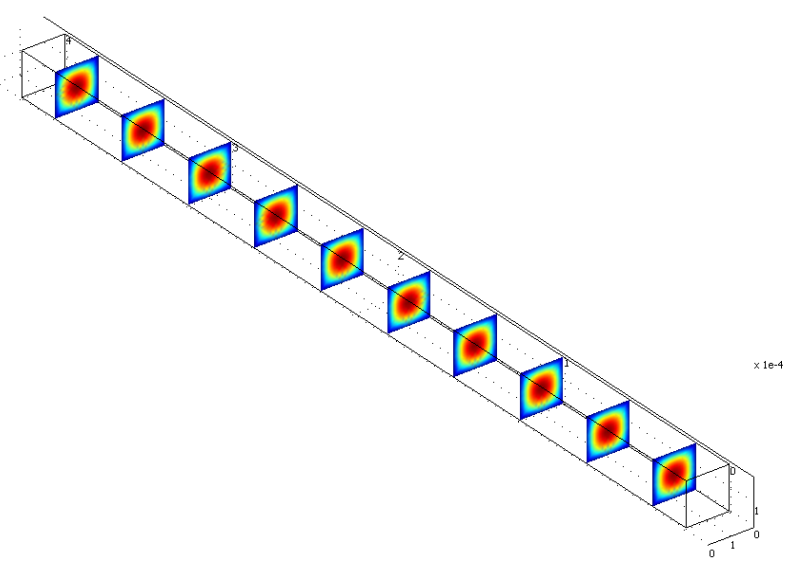

Figure 67 - Slices showing fluid flow along channel of parabolic profile channel

This model follows fluid dynamic theory that the walls will experience high stress and low flow rates while the center experiences high flow rates and low stress. To visualize this 3 dimensionally instead of by a color spectrum, the following was created. 


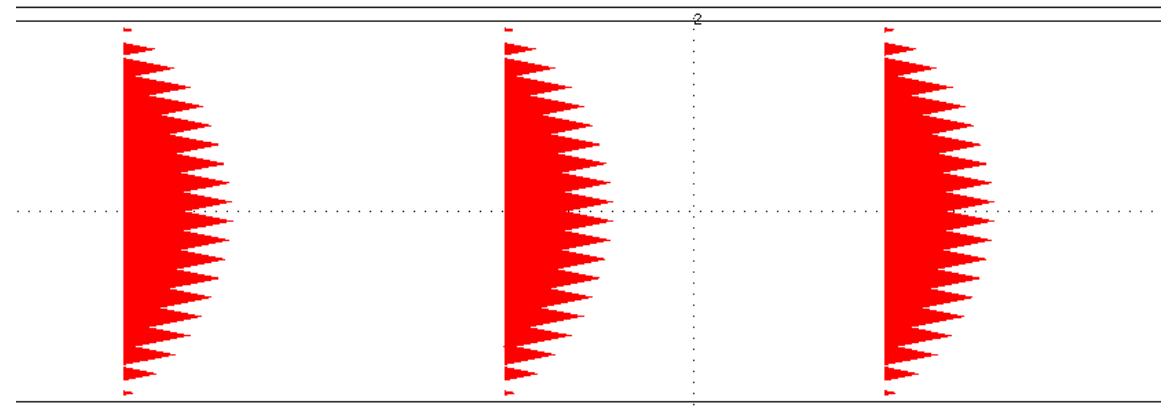

Figure 68 - 3-dimensional velocity field arrows of square profile channel in COMSOL

Viewing the velocity graphs from right angles is important when comparing the two geometries. The following shows the velocity profiles of the square channel from the top. Flow rates around the edges are very nearly 0 , so posting a graph from the absolute edges does not show details about velocities. The left graph is taken from the center of the channel and the right is taken from $1 / 3$ of the way into the channel, or roughly 65 microns.

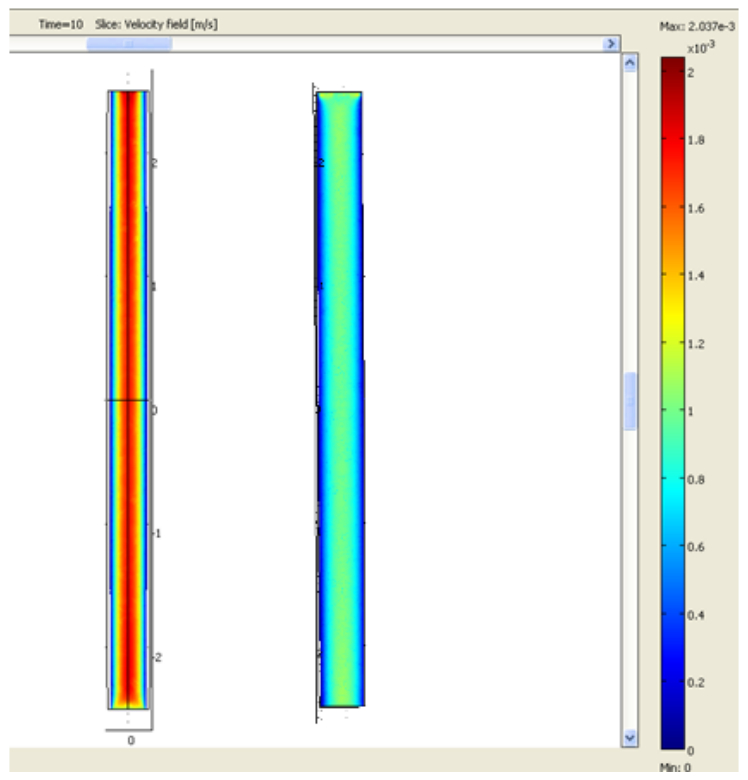

Figure 69 - Top-down view of velocity profile of square channel from $(\mathrm{L})$ middle of the channel and (R) 70 microns into the channel 
A top-down view of the square profile will give the same result as a side profile so one was not created. After viewing profiles it is important to see the numbers behind the graphs so that the different profiles can be compared numerically. The following is a graph of the fluid velocity traveling through the middle of the channel taken at a line perpendicular to the flow.

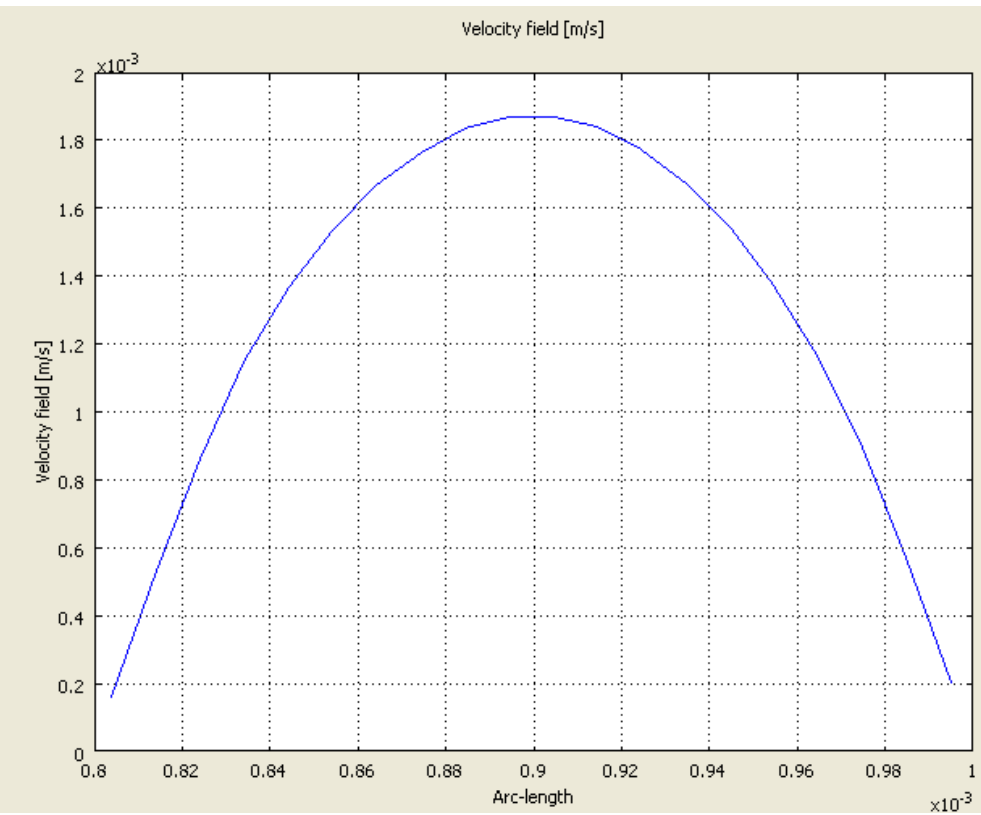

Figure 70 - Graphical velocity profile of flow through the center of the square profile channel

Recall that the fluid velocity entering the channel was $1 \mathrm{~mm} / \mathrm{s}$. The flow at the center is nearly double that at $1.89 \mathrm{~mm} / \mathrm{s}$. The graphs look fairly smooth and even but the evenness of the flow can be indicated by a graph showing the velocity down the center of the channel through its fastest streamline 


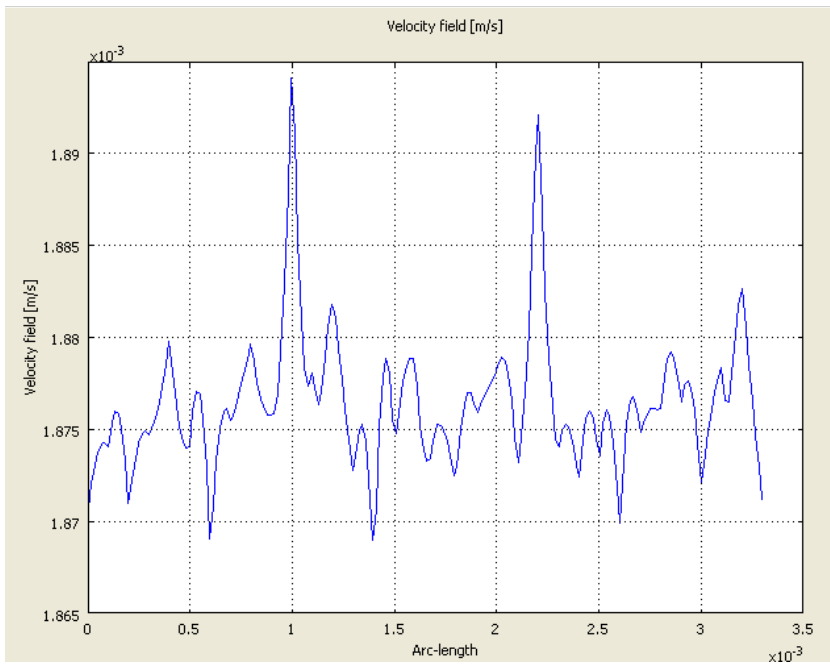

Figure 71 - Velocity along centerline of square profile channel The graph looks somewhat chaotic but the values can be contained between 1.895 and $1.865 \mathrm{~mm} / \mathrm{s}$, a small width. Just for comparison, the voltage profile through the channel was graphed.

The conductivity of the solution was high enough to create an even distribution of voltage across the channel as expected.

\subsubsection{Parabolic Profile Modeling}

An ellipsoid was created with a 176 micron minor axis and a 120 micron major axis to recreate the dimensions of the 5-watt vector cut channel. The same settings as the square profile model were used and a fine mesh was created out of the model that gave the following model. 


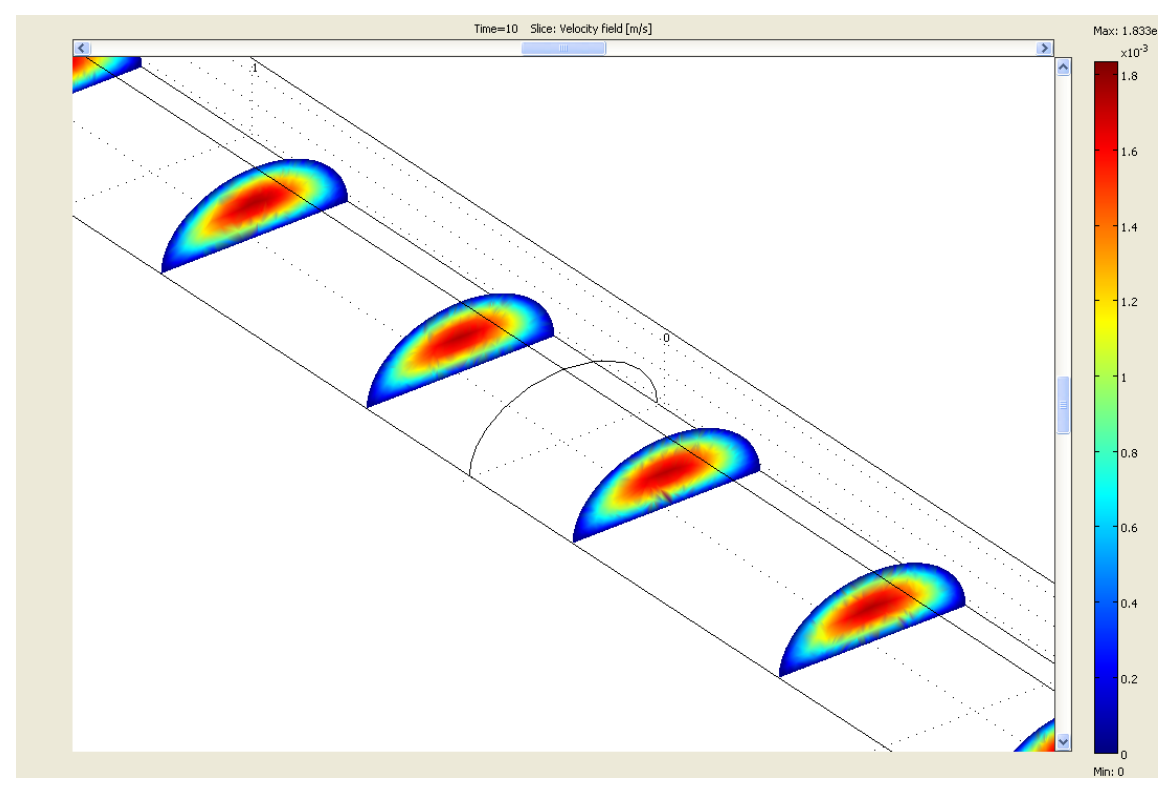

Figure 72 - Flow velocity slices of a parabolic channel

Already a difference can be seen between the square profile and the parabolic profile in the transition areas between colors of the spectrum indicating flow velocity. The flow lines are not as even and concise as with the square profile. To visualize the flow rates, an arrow plot was created to show velocities in the channel.
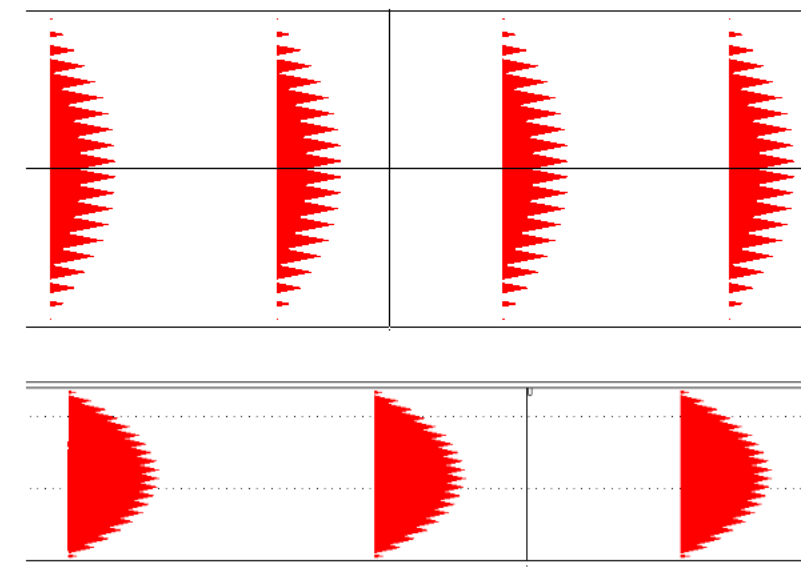

Figure 73 - 3-dimensional velocity field arrows of parabolic profile channel, (T) top down view, (B) side view 
The top-down view of the parabolic graph is nearly identical to that of the square profile yet the side view shows a shortened version of the graph with a more dramatic drop-off near the edges. To keep scale in mind, the graphs of the velocities were created. The first graph was created to show the values from the top of the parabolic channel to the bottom to compare against the square channel.

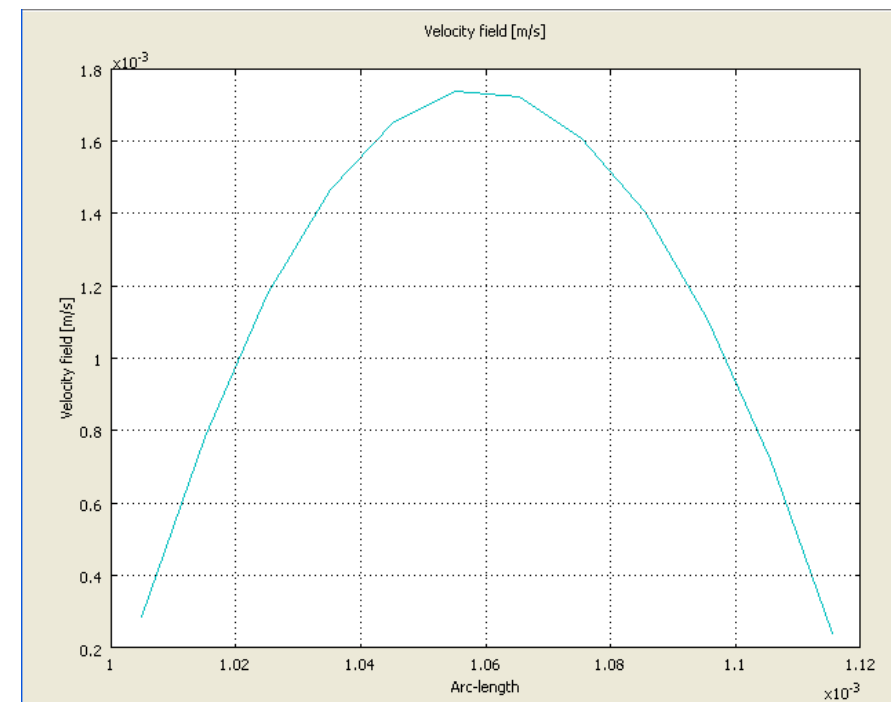

Figure 74 - Velocity field from top to bottom of parabolic channel

The velocity reaches its maximum at roughly $1.75 \mathrm{~mm} / \mathrm{s}$; not as high as the maximum experienced by the square profile channel but very close. It can then be concluded that the side view of the parabolic channel is merely a 'squeezed' version of the square channel. The main difference between the two channel geometries then is the velocity starting at the top of the channel and taking slices across the channel moving towards the bottom. The first graph was taken 20 microns from the top because as before the velocity at the very top of the channel is very nearly 0 . 


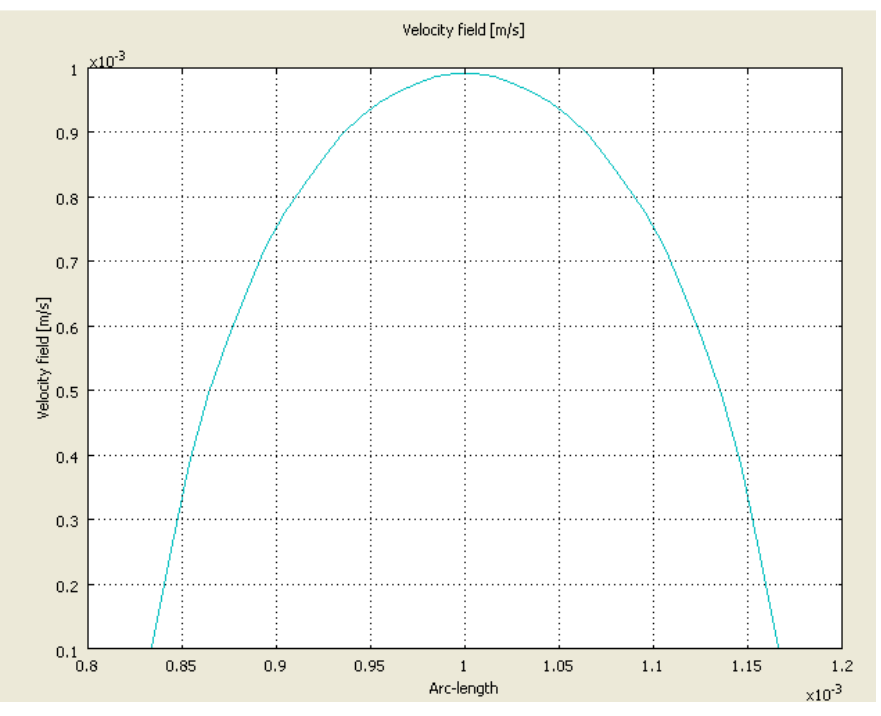

Figure 75 -Velocity graph across the parabolic profile channel 20 microns below the top

This parabolic graph looks very similar to the other velocity graphs, yet only reaching a maximum of nearly $1 \mathrm{~mm} / \mathrm{s}$. Another slice must be taken to compare.

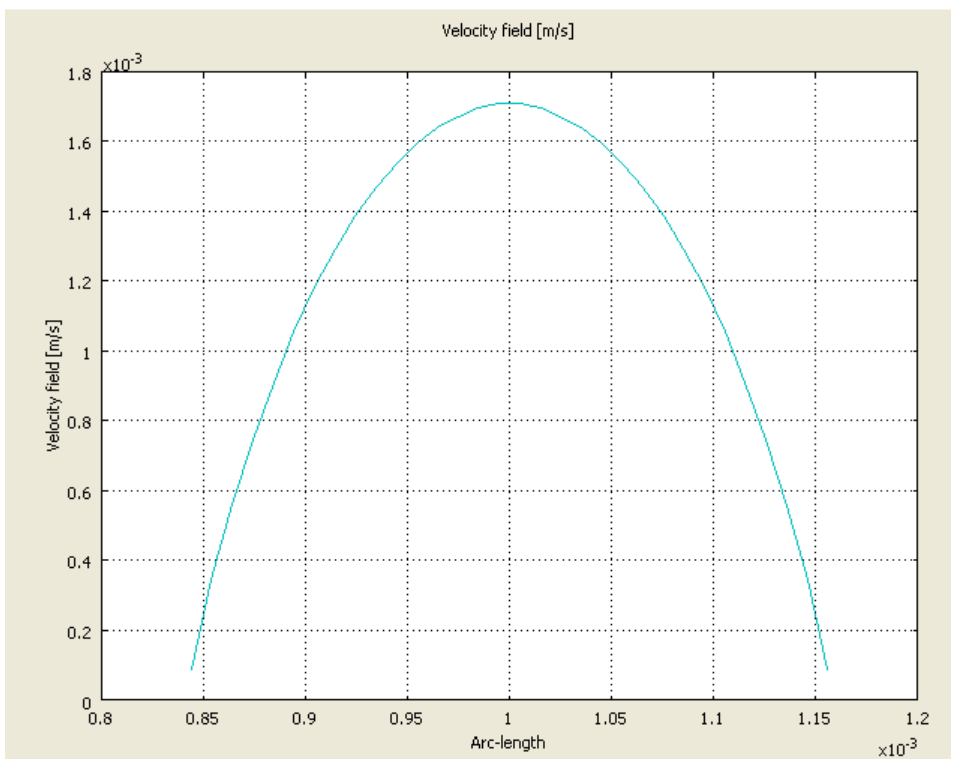

Figure 76 - Velocity graph across the flow in a parabolic profile channel 50 microns from the top 
This graph represents the velocity profile roughly halfway between the bottom and the top of the parabolic channel and reaches a maximum of $1.7 \mathrm{~mm} / \mathrm{s}$ or very close to the maximum experienced by the parabolic channel.

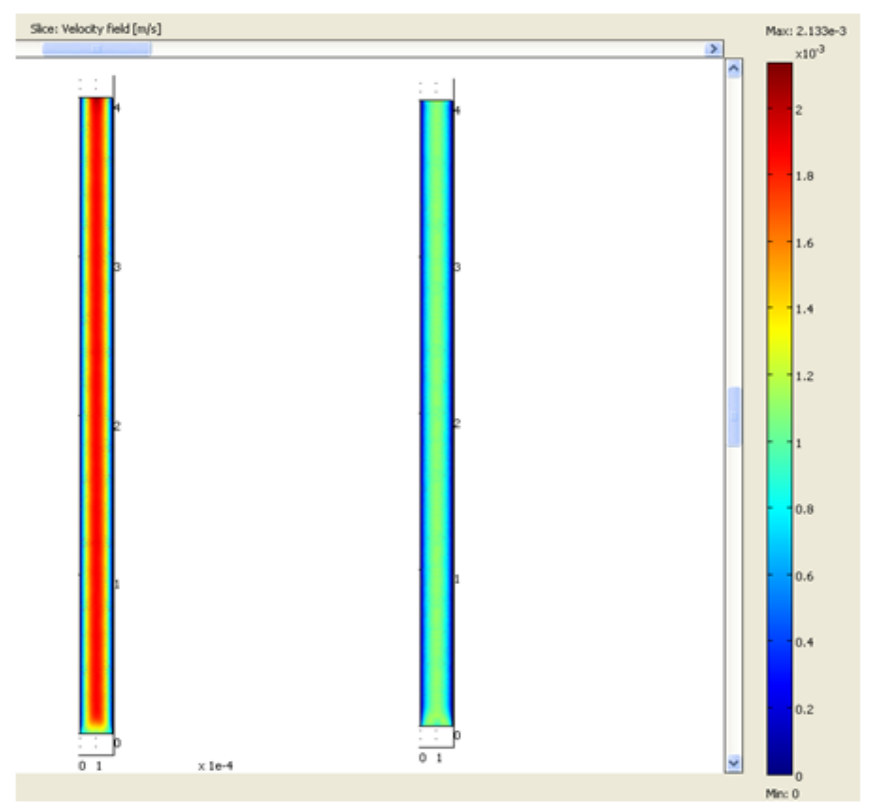

Figure 77 - Top-down view of parabolic profile with (L) halfway down the depth of the channel and (R) 1/3 of the way into the depth of the channel

The top-down graphical view of the velocity field is very similar to that of the square profile channel although this graph lacks the dark red stripe down the center indicating the fastest fluid flow on the graph. The fastest flow is epxerienced only at the inlet and quickly dissipates. 


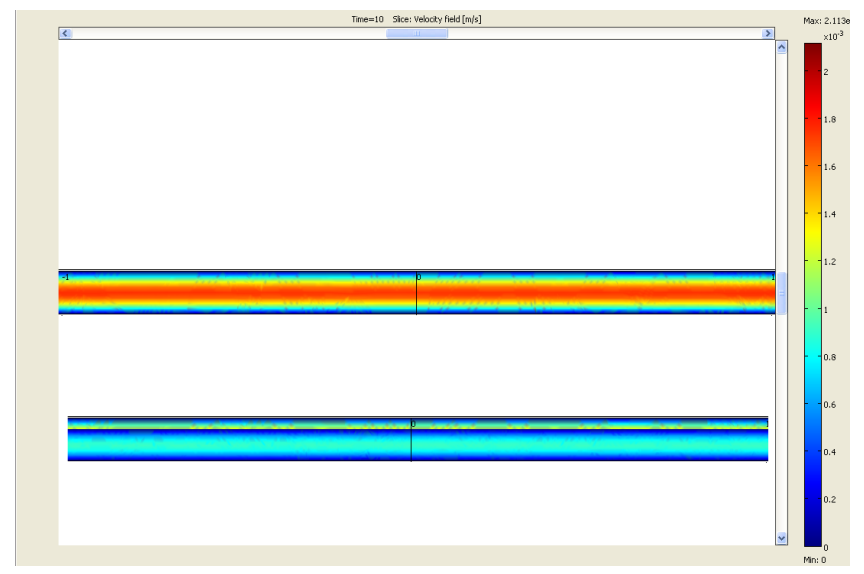

Figure 78 - Side view of velocity graph with $(T)$ middle of the channel and (B) 1/3 of the way into the channel from the side

The side view of the parabolic channel is again similar to the square profile minus the presence of a red stripe of high velocity fluid flow in the center. The graph showing the velocity field $1 / 3$ the distance from the edge is different however in that it experiences slower velocity than is found in the square channel, indicated by the absence of a strong green stripe in the center. Lastly, a graph was created to compare the maximum velocity profile down the length of the channel through the maximum velocity streamline against that of the control channel.

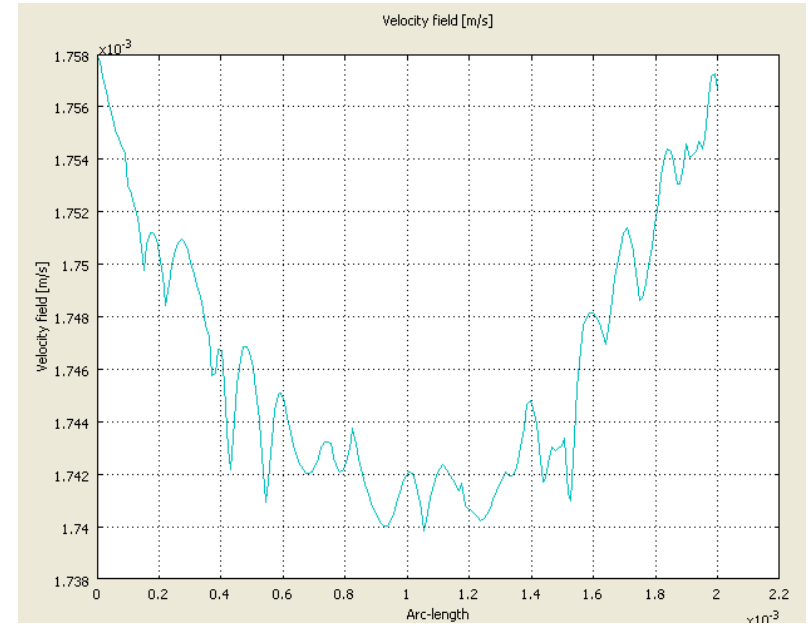

Figure 79 - Velocity field down center of parabolic profile channel in area of highest fluid velocity 
Although the profile may be surprising, the parabolic path that the graph roughly follows can be attributed to the slow parabolic arc taken by the bottom of the ellipsoid used to create the CAD model. Taking this into account and analyzing the distribution of points along the parabola, the apparent randomness of the data still falls within a much smaller bound than the \pm .015 range of the square profile channel. Perhaps the smaller range of total velocity experienced across the channel leads to less chaotic flow down the streamlines.

\subsubsection{Concluding Remarks}

The results from the electroosmotic flow models show that the parabolic profile model is actually superior to the square profile channel due to the more evenly distributed electroosmotic velocity. The models of pressure driven flow results to those of the control models. The parabolic profile graph indicates that the flow rates will be slightly less than those of the square profile but the profiles are similar enough that the two profile geometries can be used for the same experimentation without a large amount of variation. One important idea to take from this model is the data collection width. The velocities at the edges drop off by a significant amount, and data should only be collected from spheres that appear within the middle $80 \%$ of the channel. 


\section{Results}

This section details the results of both laser etching and electrophoresis experiments.

Laser etchings were performed with increasing laser powers. If the terminology is not clear, vector cuts are single passes of the laser that yield a parabolic channel. Raster cuts are juxtaposed laser passes with a stepover of approximately 25.4 microns to create a recess of roughly the same depth. The axes on the graphs of laser etched channels are scaled to give a sense of the actual geometry of the channel.

\subsubsection{Channel Geometry of Different Laser Powers}

Laser cuts were made with a Universal Laser Systems X2-660 with a 100-watt $\mathrm{CO}_{2}$ laser source. The laser was guided by AutoCAD drawings of the chips and the printer driver provided by Universal Laser Systems. The extruded PMMA sheets were supplied by McMaster Carr at thickness of 0.25 ". The sheets came with a thin plastic backing that was removed just prior to laser cutting. After laser cutting, the PMMA was cleaned with a 70\% ethanol solution and then DI water. The samples were kept in a sealed container until analysis with the profilometer.

The profilometer, Ambios Stylus Profilometer model XP-1, is capable of analysis 1mm long along one axis. The probe was set at a point close enough to the channel to capture the entire geometry of the etched channels. 


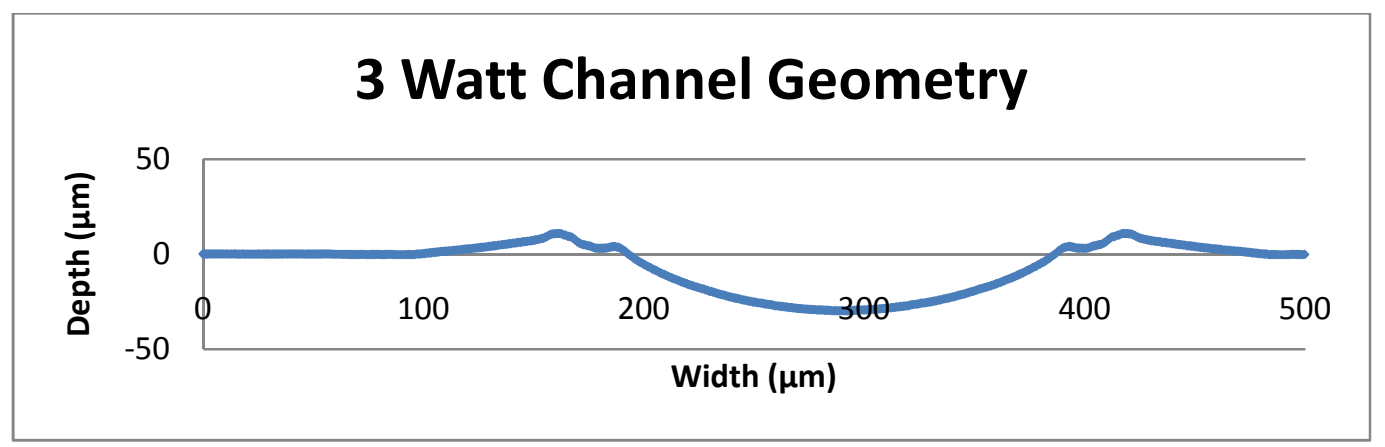

Figure 80 - 3 Watt channel geometry. The 3 watt geometry was corrupted during the analysis stage. The above data was taken from a 3 watt raster profile 10.6 micron wavelength $\mathrm{CO}_{2}$ and mirrored to create geometry like that of a channel. The depth was adjusted based on ratios of channel to raster depths of other laser powers with an increase of $10 \%$.

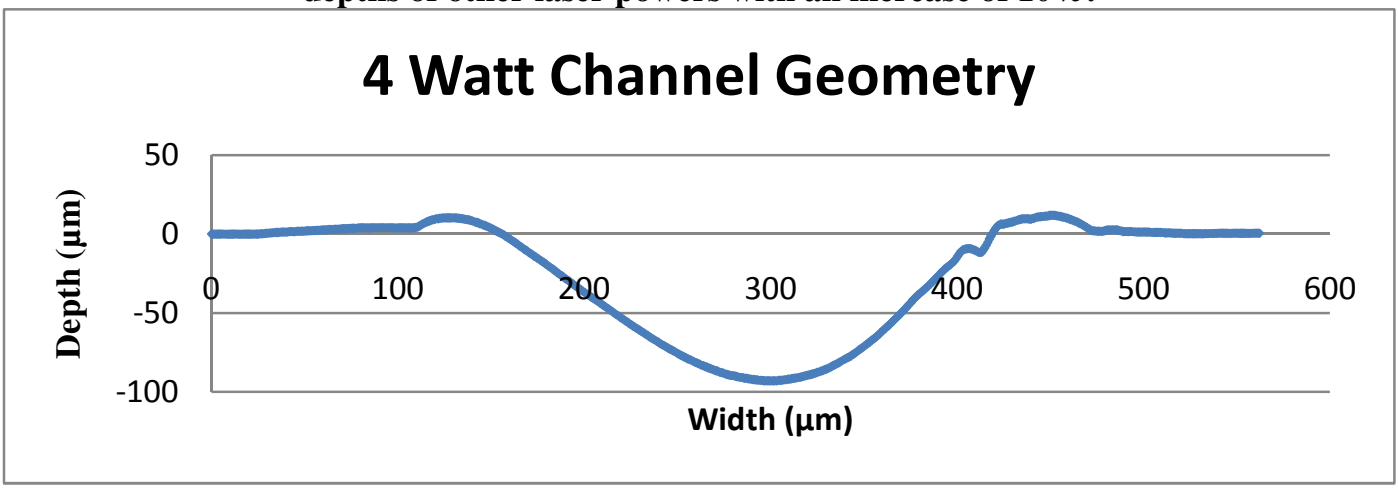

Figure 81 - Channel geometry of a single pass with a 4 watt 10.6 micron wavelength $\mathrm{CO}_{2}$ laser. The 0 Y-axis value is associated with the untouched PMMA surface.

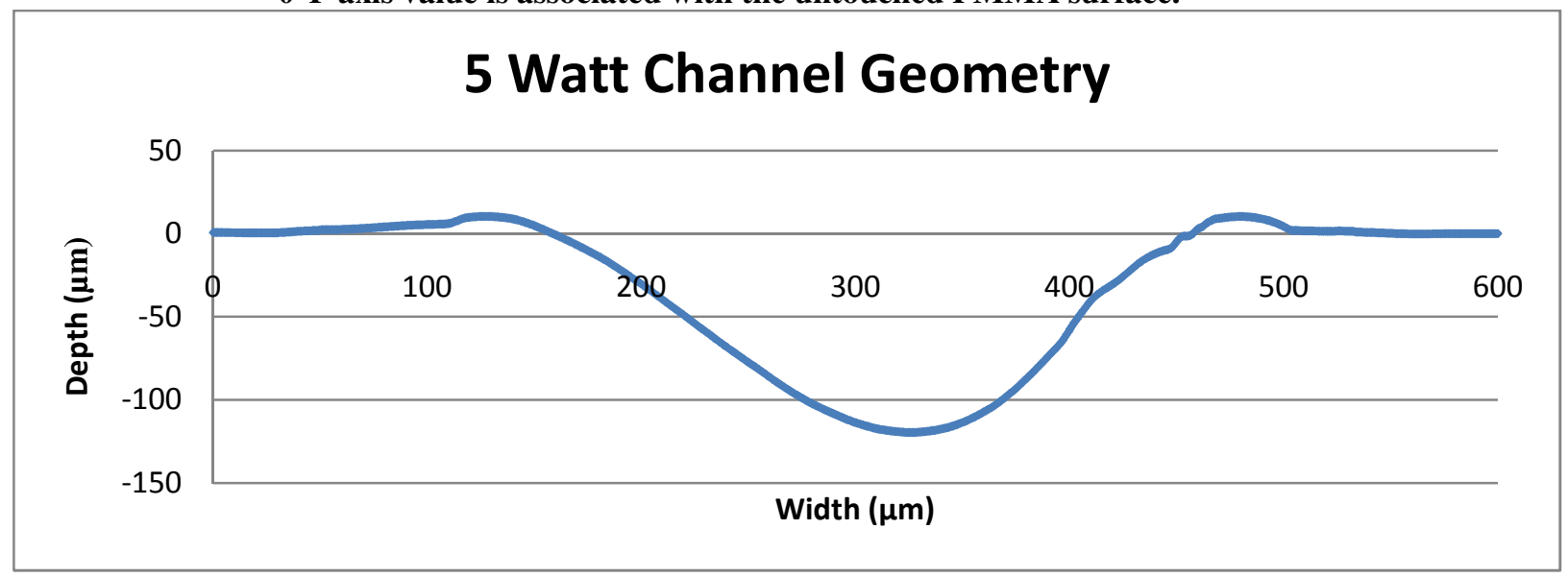

Figure 82 - Channel geometry of a single pass with a 5 watt 10.6 micron wavelength $\mathrm{CO}_{2}$ laser. The $0 \mathrm{Y}$-axis value is associated with the untouched PMMA surface. 


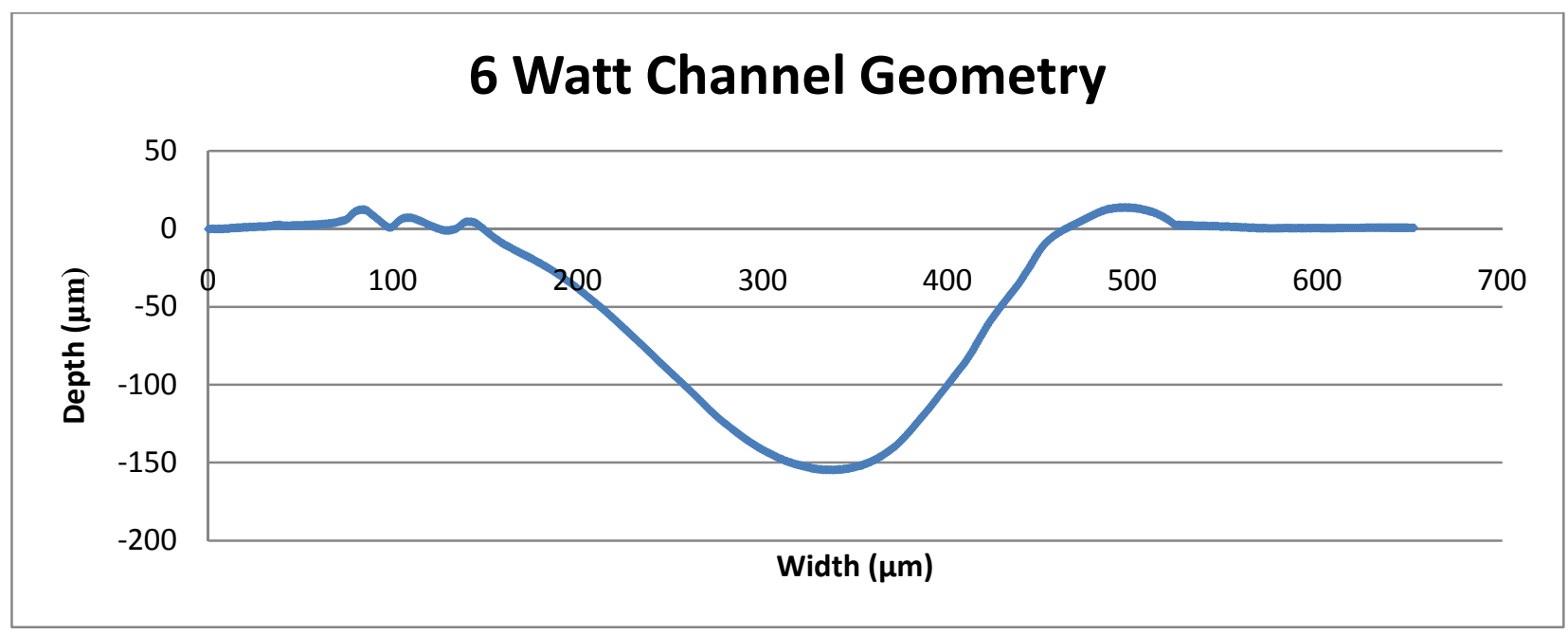

Figure 83 - Channel geometry of a single pass with a 6 watt 10.6 micron wavelength $\mathrm{CO}_{2}$ laser. The 0 Y-axis value is associated with the untouched PMMA surface.

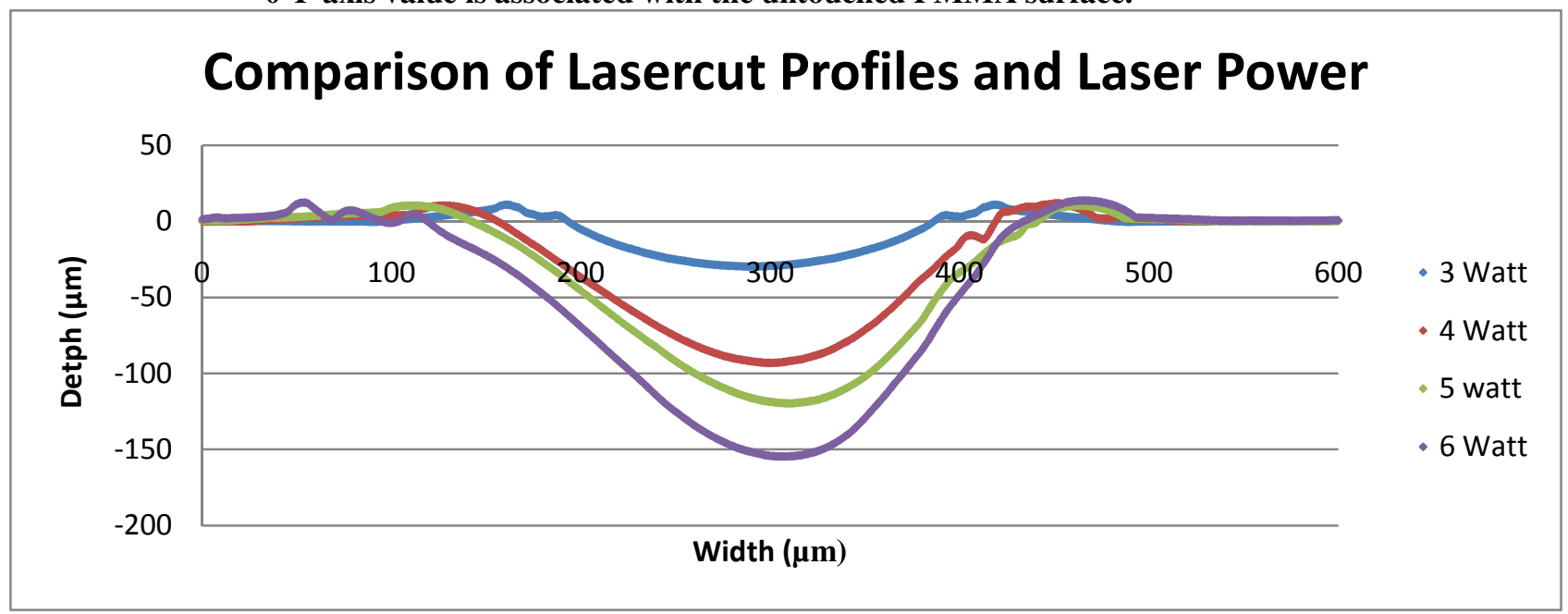

Figure 84 - Comparison graph of channel geometries etched with different laser powers. The 0 Yaxis value is associated with the untouched PMMA surface.

Very simply, increasing laser power yields increasing channel depth. Further analysis of the results can be found in the discussion section. 


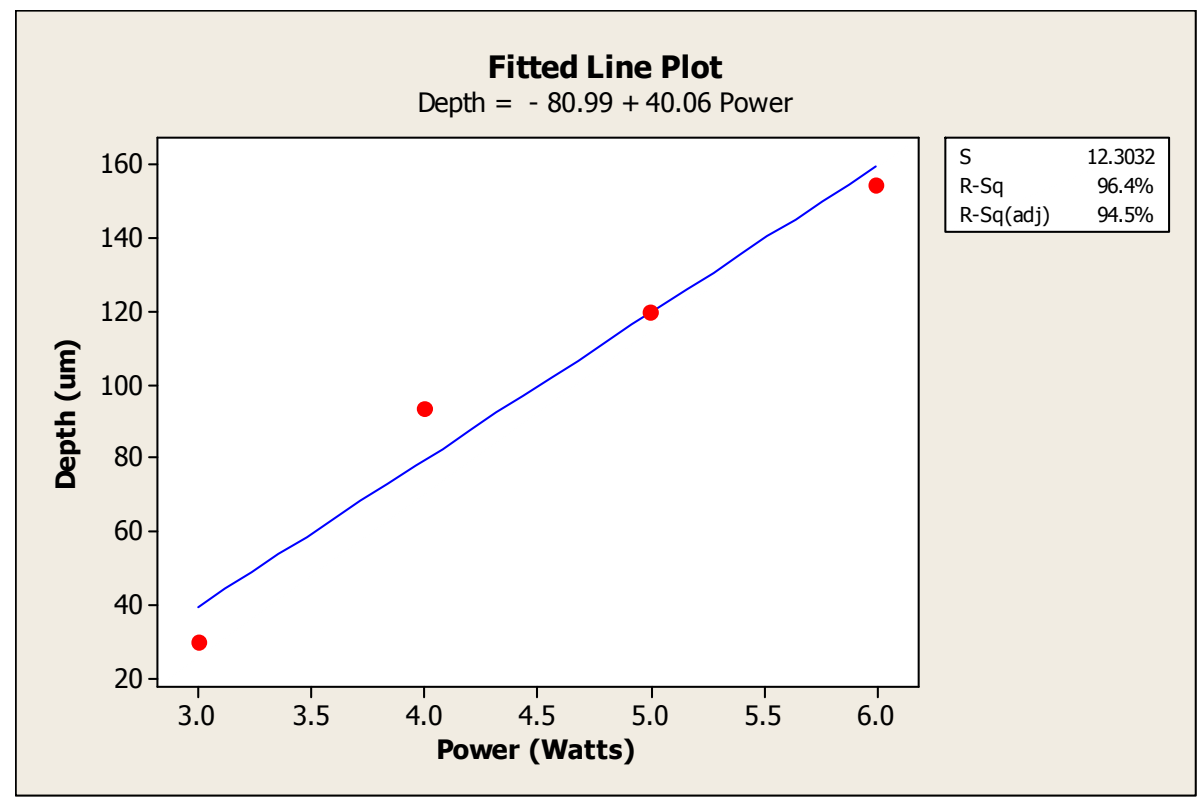

Figure 85 - Graph comparing depth of channel versus laser power. Linear line of best fit with associated equation and $R^{2}$ value are shown.

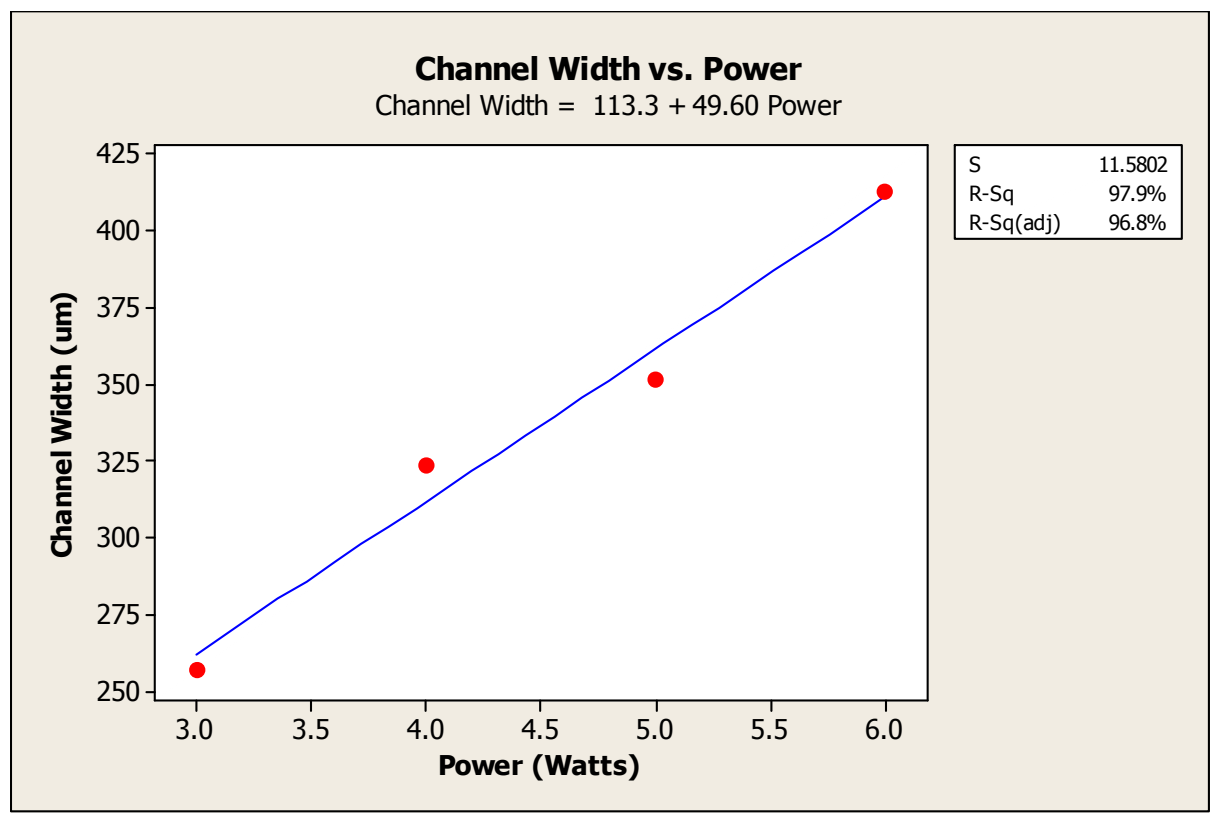

Figure 86 - Graph comparing channel width to laser power. Linear line of best fit with associated equation and $R^{2}$ value are shown. 


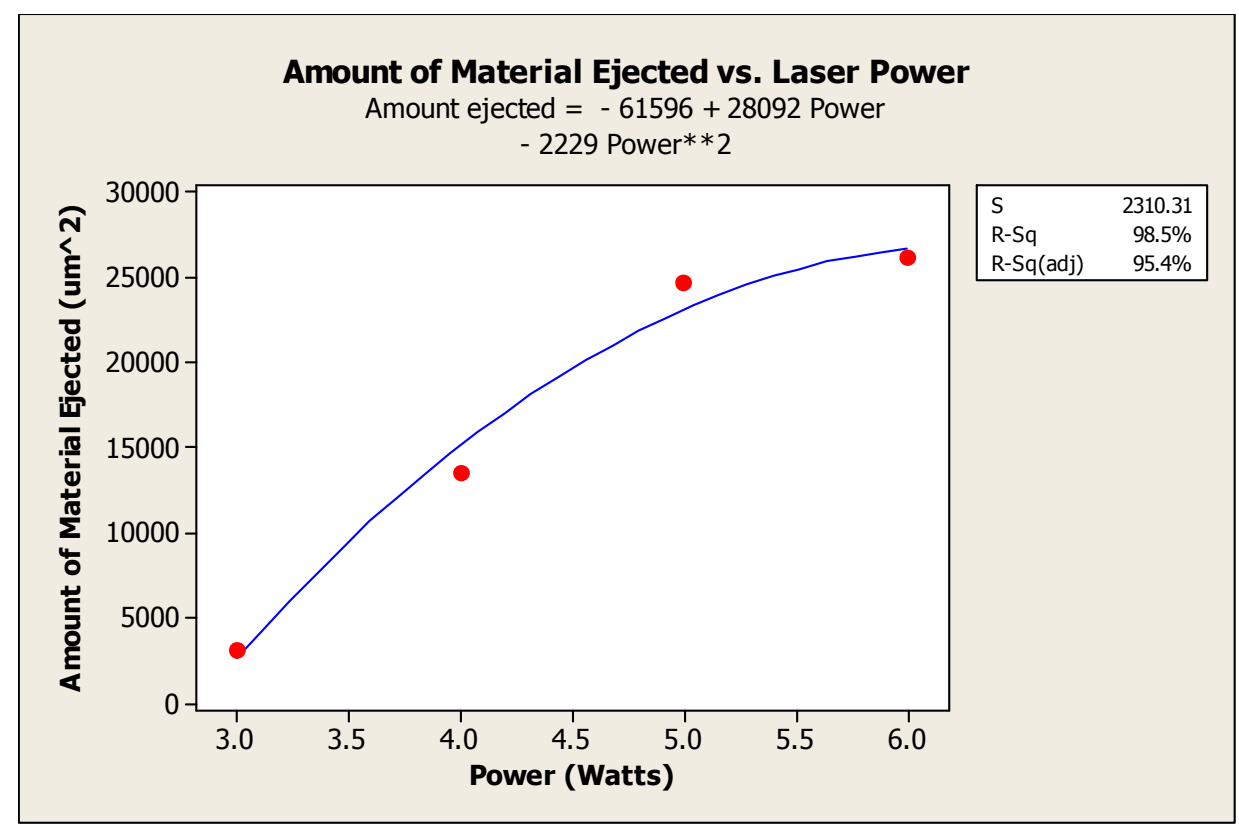

Figure 87 - Graph comparing ejected material versus laser power with quadratic line of best fit. The values were found by integrating with a left Riemann sum. Polynomial line of best fit with associated equation and $R^{2}$ value are shown.

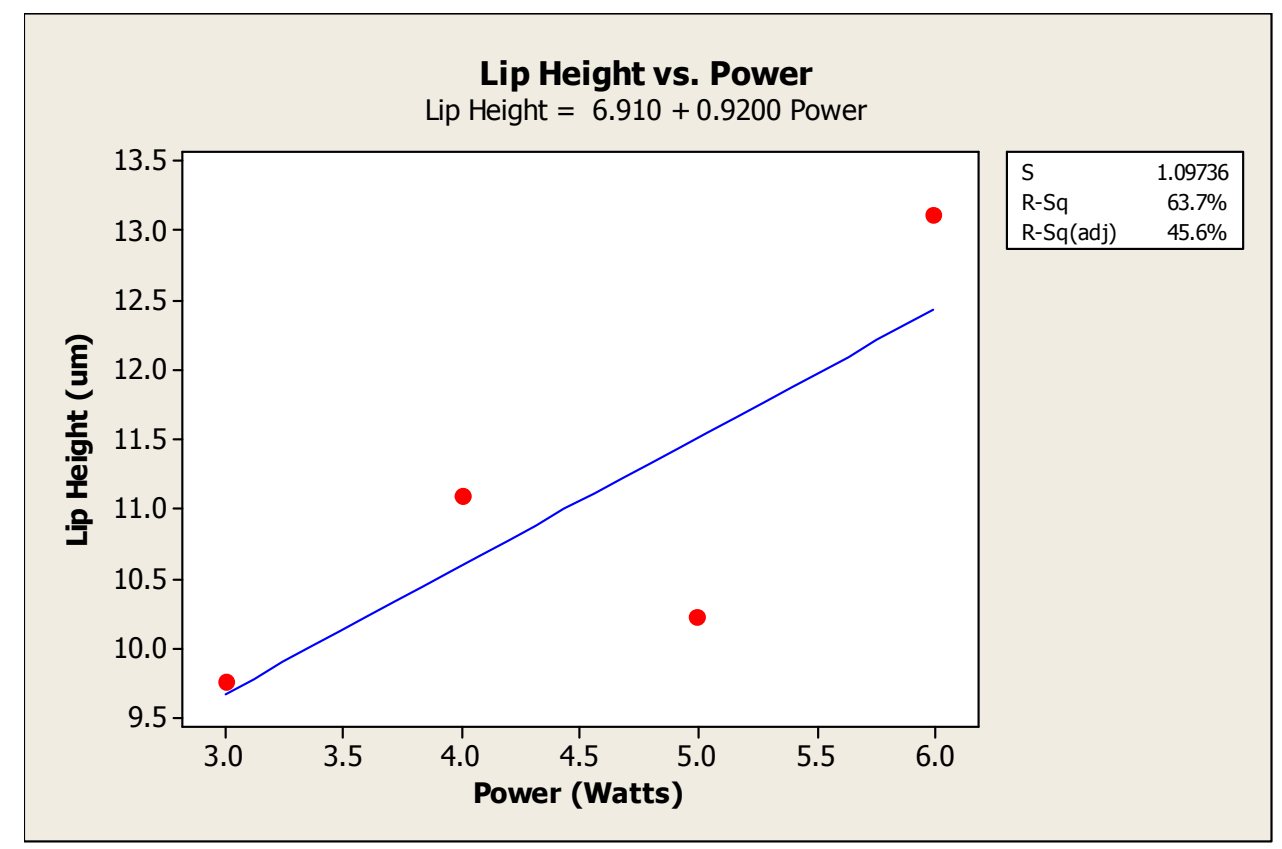

Figure 88 - Graph comparing lip height versus laser power with linear line of best fit. Linear line of best fit with associated equation and $R^{2}$ value are shown. 


\subsubsection{Comparison of Untreated and Heat-Treated Channel Geometry}

Heat treatment involved placing the chips in Quincy Lab oven, model 10GC, at 100

$( \pm 5)^{\circ} \mathrm{C}$ for 1 hour and then removed and cooled at room temperature. The same

profilometer was used for the post-heat treatment. Finding the exact same pre-treatment

location for data collection as post-treatment would be a complicated task, so data was

taking from simply another spot in the middle of the channel.

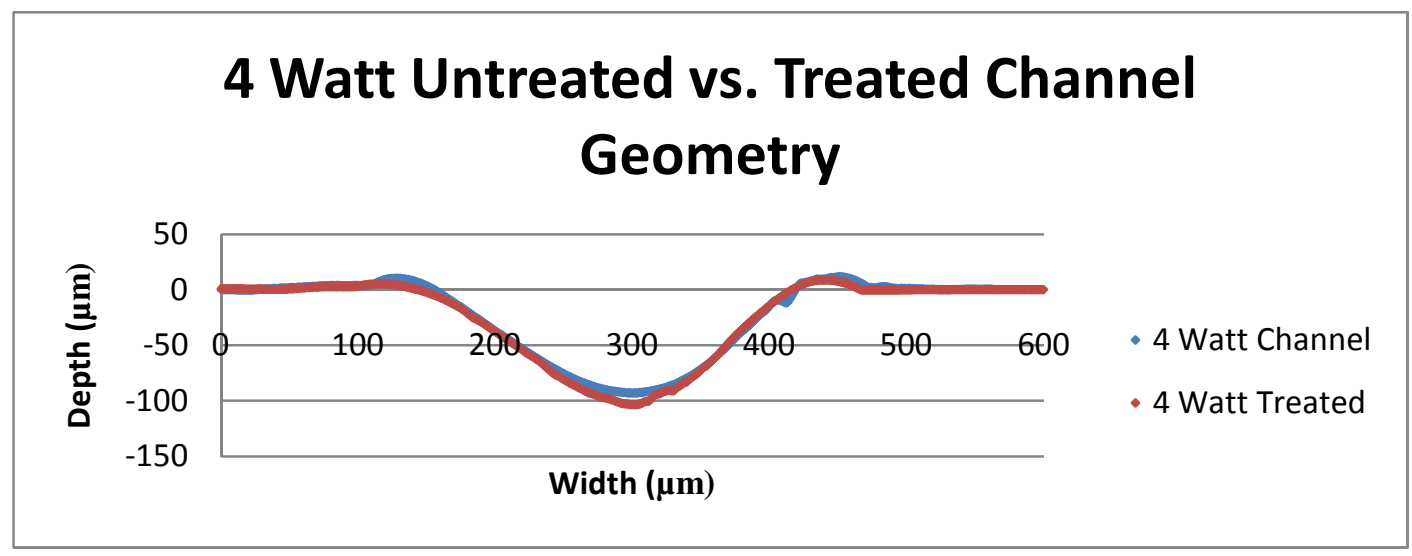

Figure 89 - Comparison of untreated and heat-treated channel geometry for a channel etched with a 10.6 micron wavelength $\mathrm{CO}_{2}$ laser at 4 watts. The blue line represents pre-treatment while red line indicates geometry post-treatment

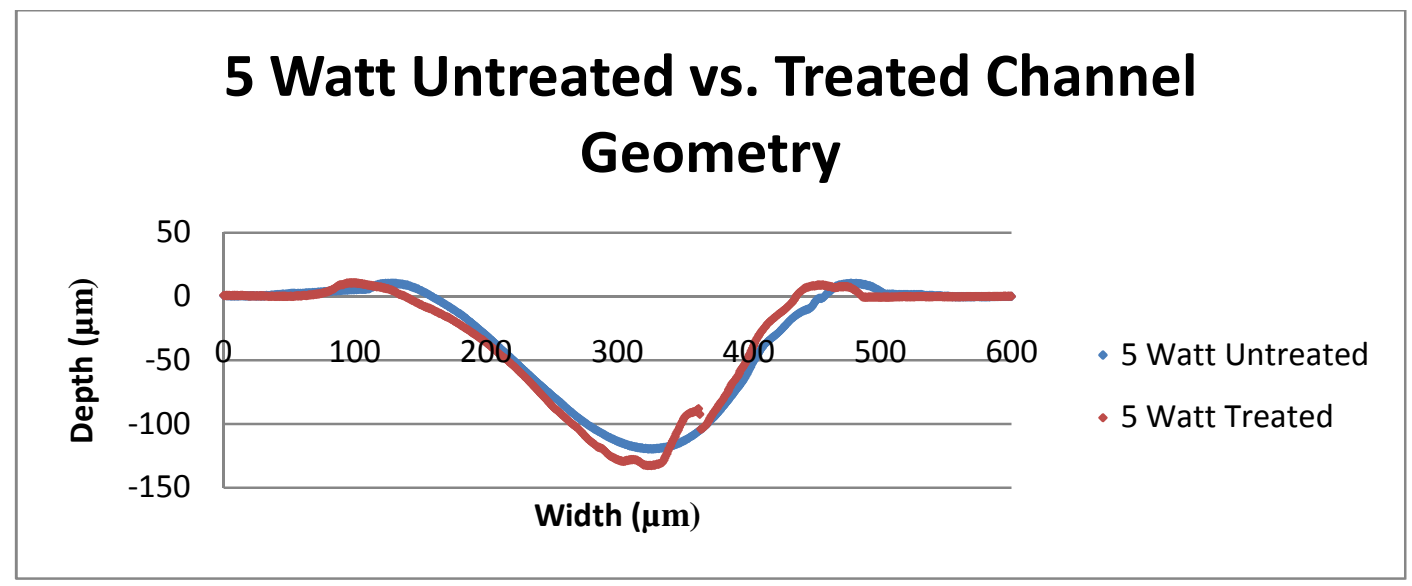

Figure 90 - Comparison of untreated and heat-treated channel geometry for a channel etched 10.6 micron wavelength $\mathrm{CO}_{2}$ laser at 5 watts. The blue line represents pre-treatment while red line indicates geometry post-treatment 


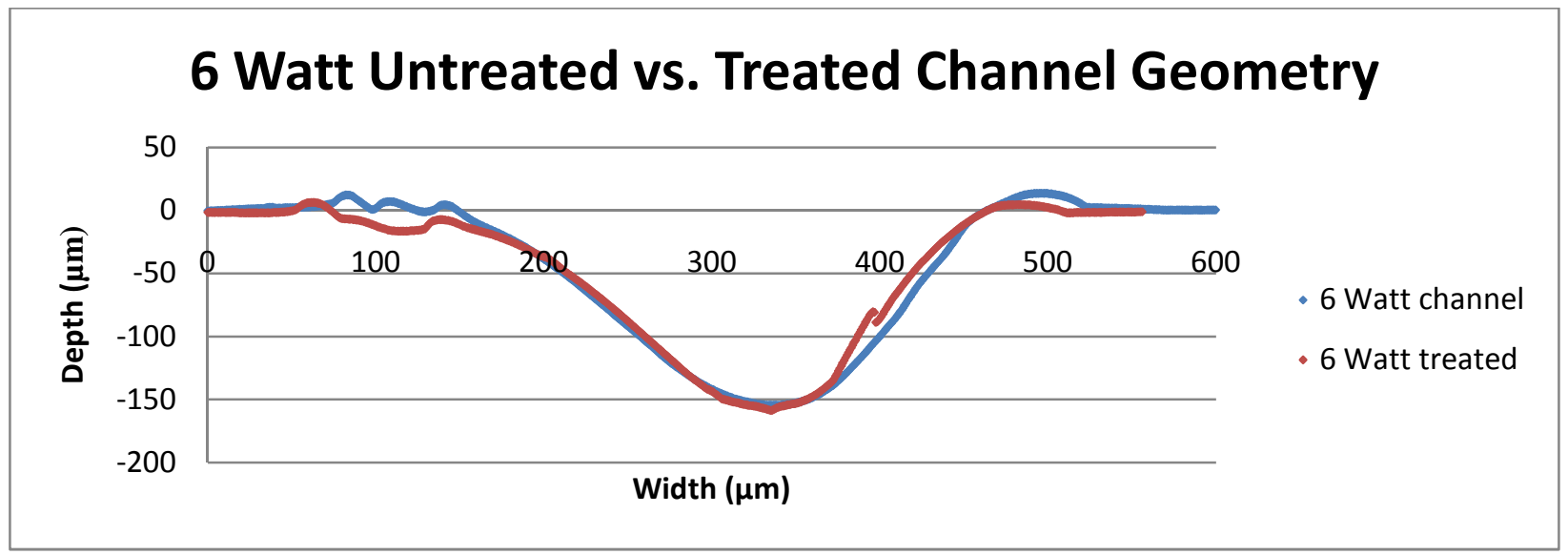

Figure 91 - Comparison of untreated and heat-treated channel geometry for a channel etched with a 10.6 micron wavelength $\mathrm{CO}_{2}$ laser at 6 watts

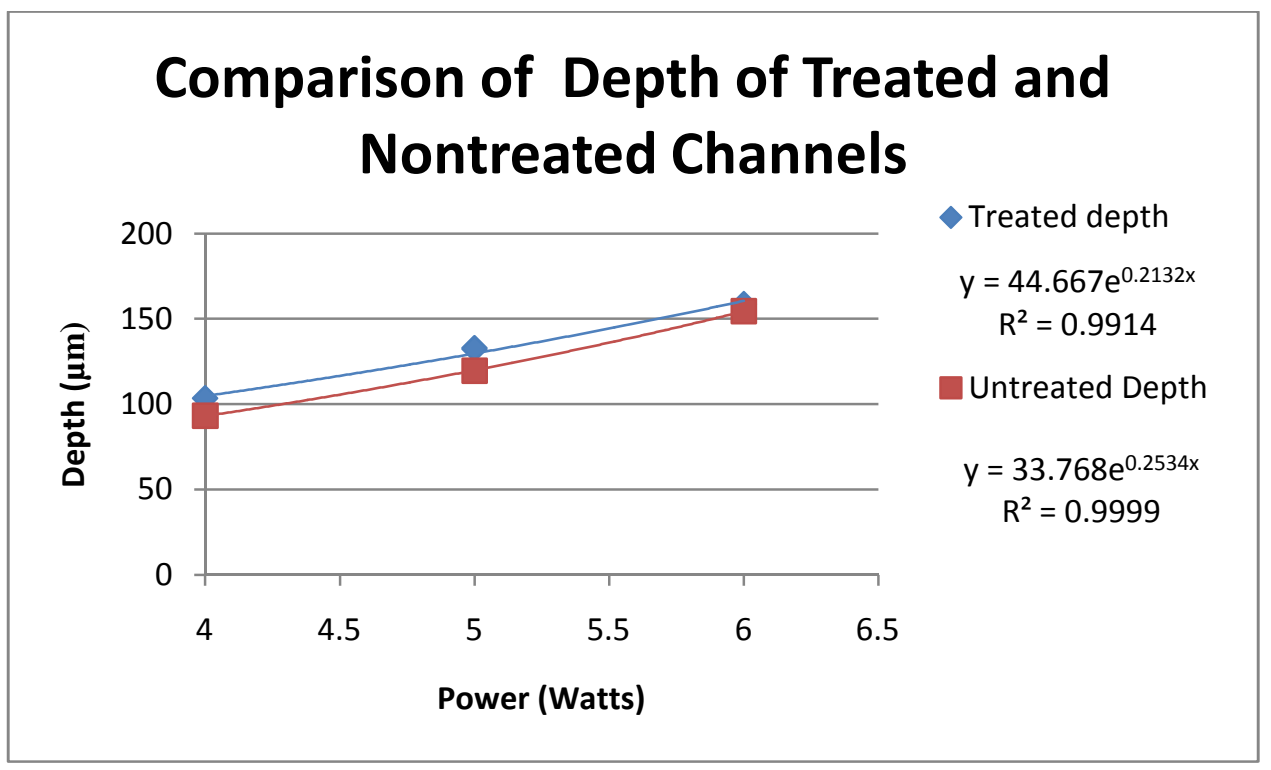

Figure 92 - Comparison of maximum depth of channels versus laser power with logarithmic lines of best fit drawn along with respective $\mathbf{R}^{2}$ values 


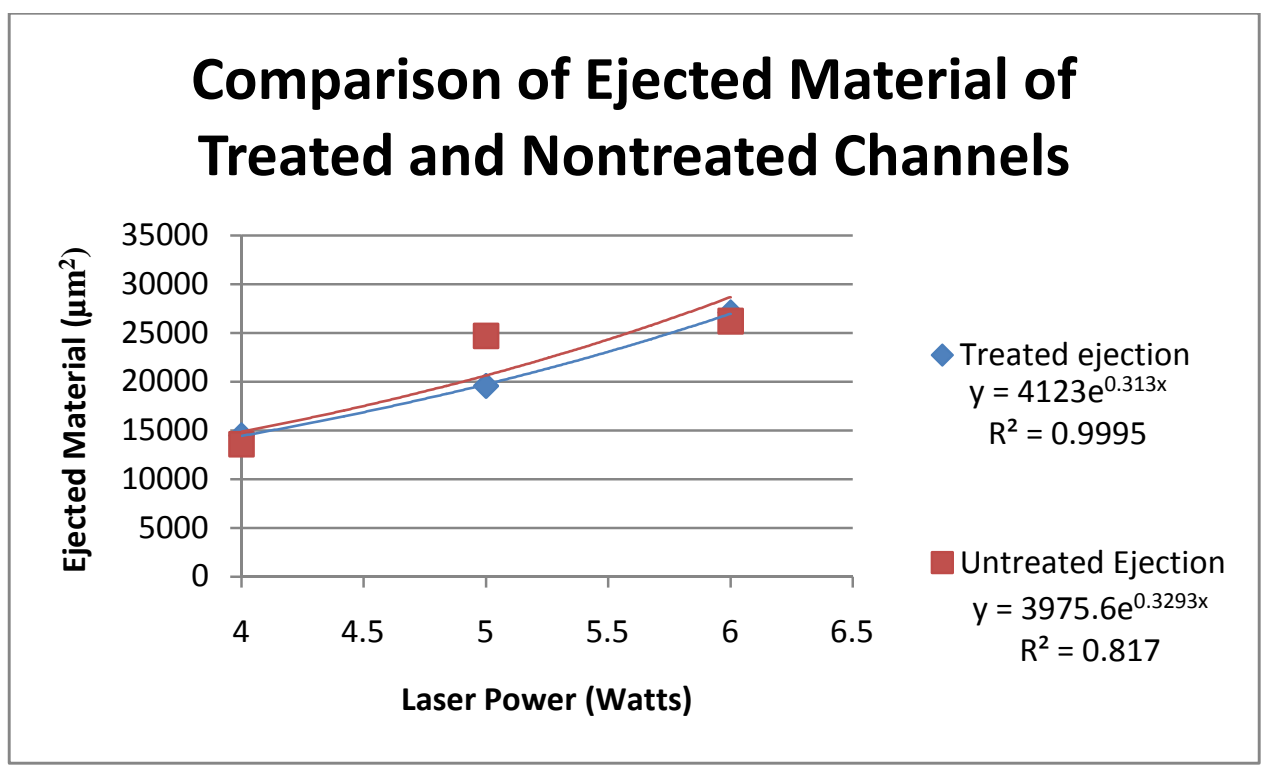

Figure 93 - Graph of ejected material from the channel versus laser power with a logarithmic line of best fit drawn. The material is not ejected from the channel during heat treatment. Rather, keeping with the naming scheme of the data the integrated sum of the depth of the treated channel was measured to compare against that of the untreated channel. The values were found by integrating with a left Riemann sum.

\subsubsection{Results for Multiple Laser Passes}

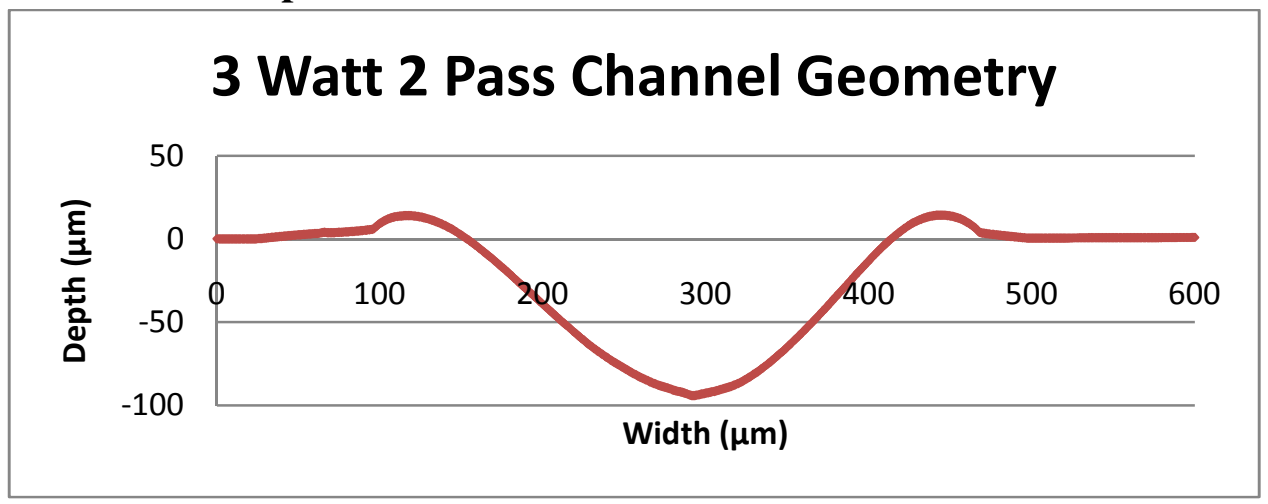

Figure 94 - Channel geometry of a channel after two passes with a 3 watt laser 


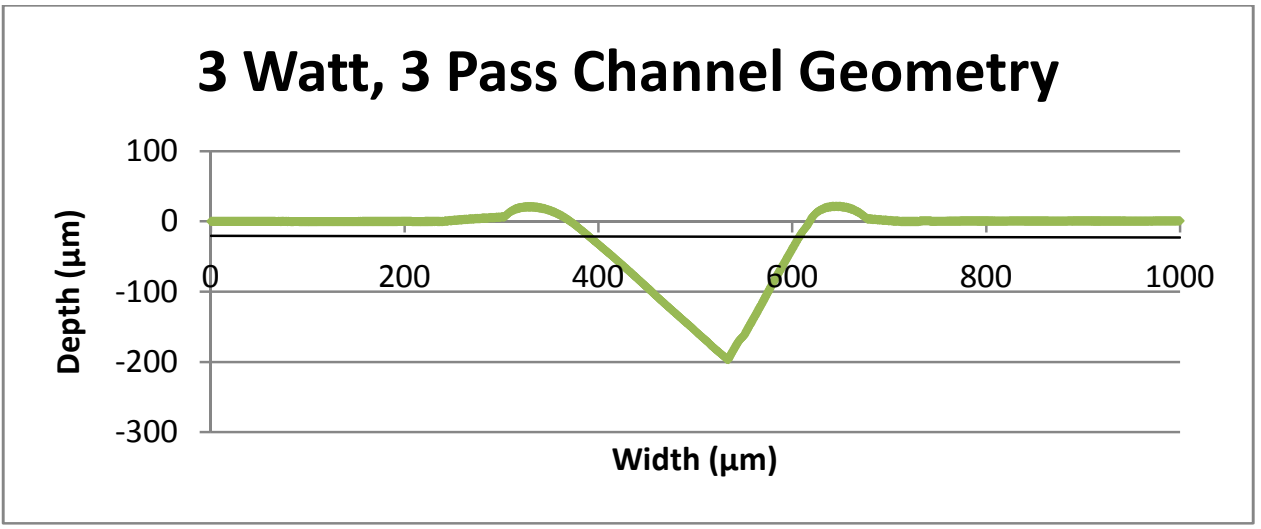

Figure 95 - Channel geometry of a channel after three passes with a 3 watt laser

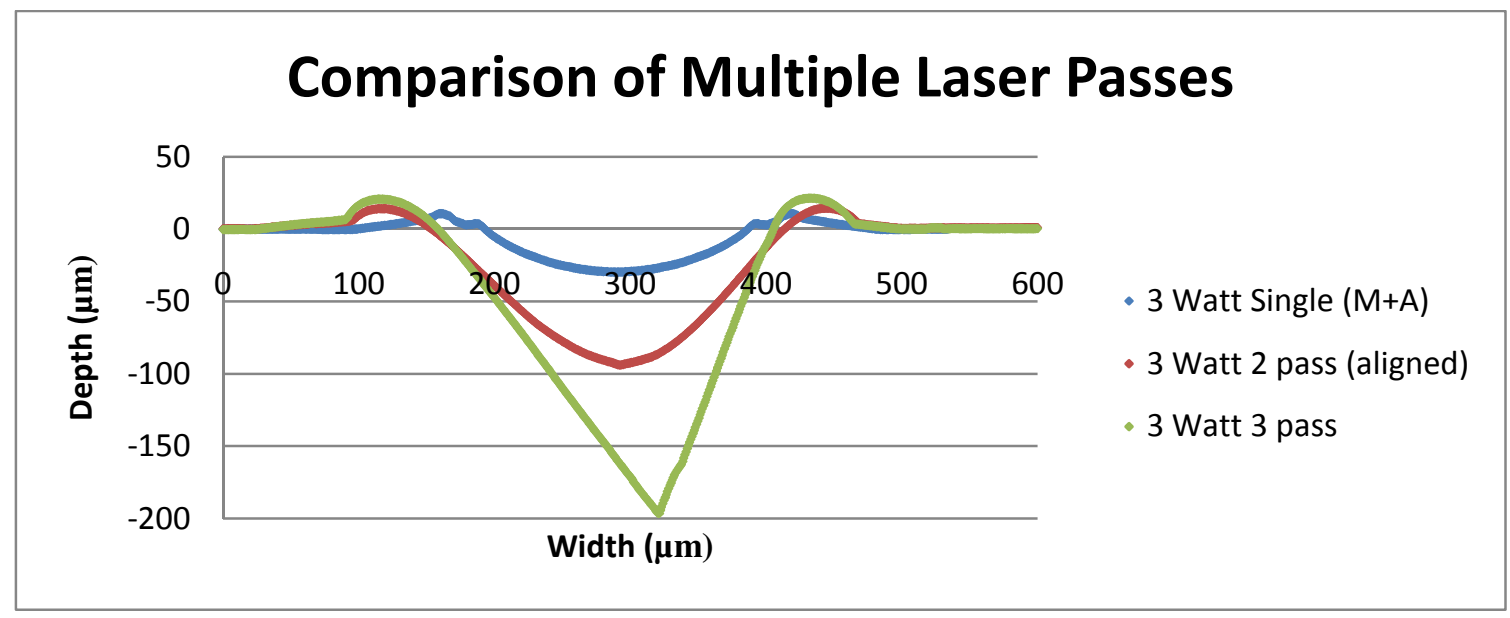

Figure 96 - Overlaid comparison of the same laser power passed multiple times over a single channel 


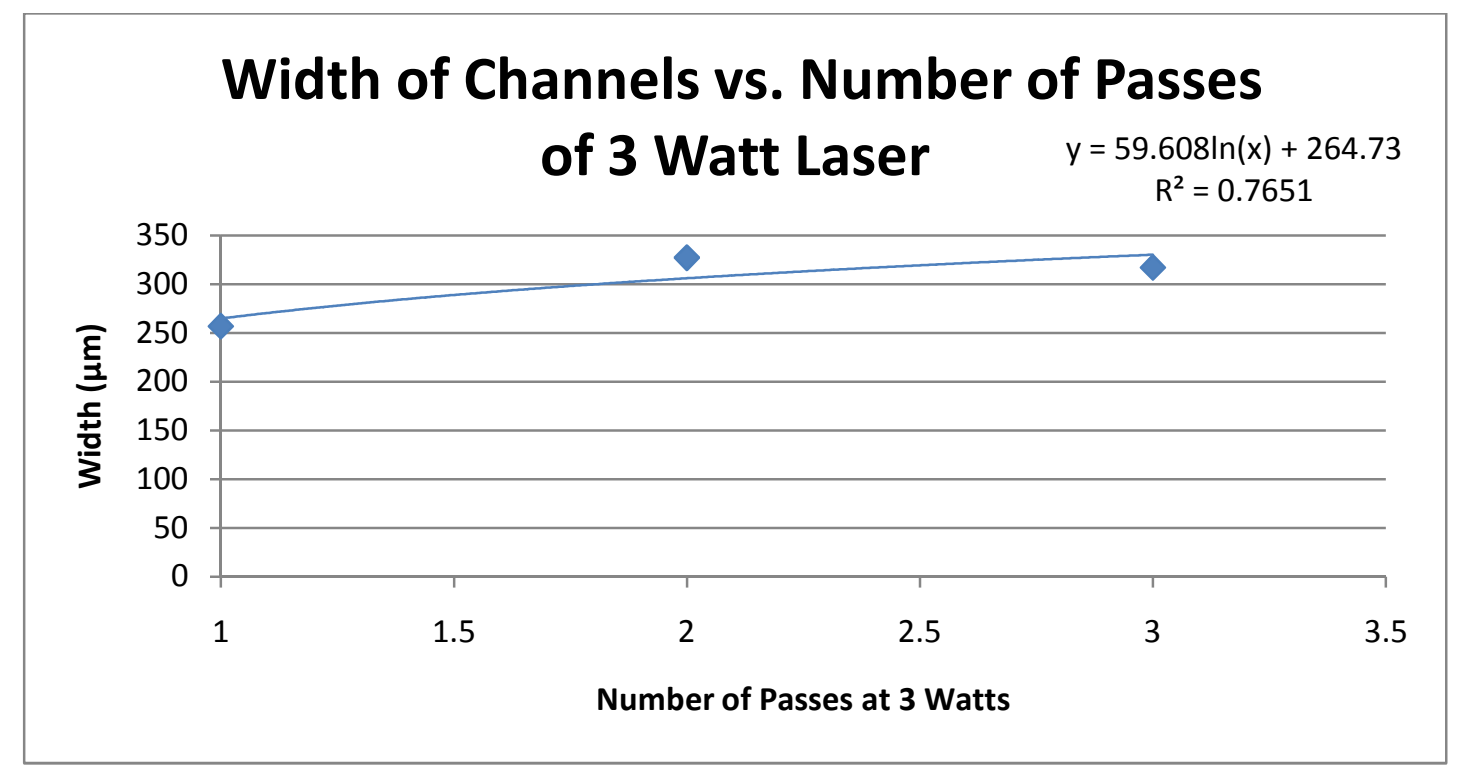

Figure 97 - Graph comparing the width of channels after multiple passes with a logarithmic line of best fit, its equation and $R^{2}$ value

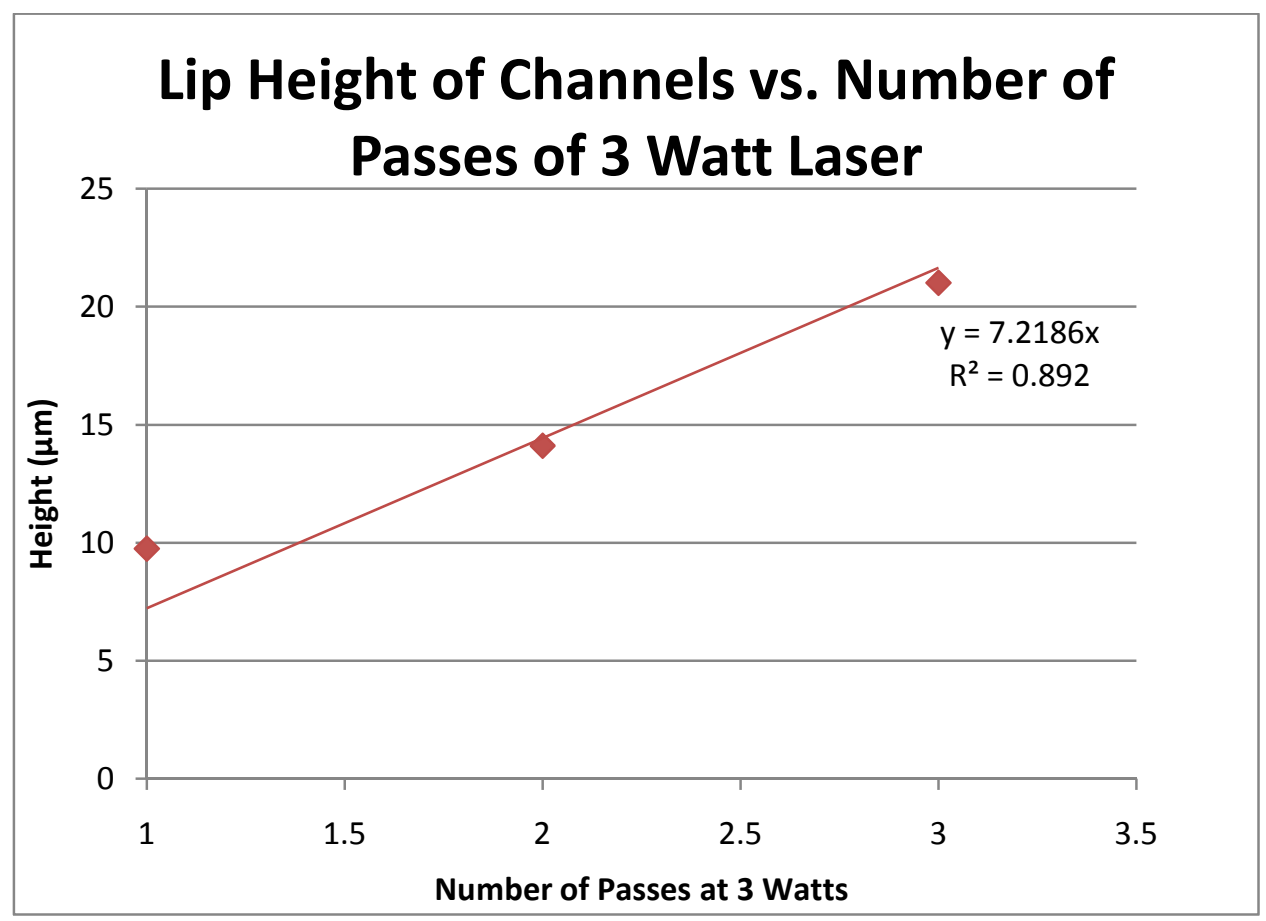

Figure 98 - Height of redeposited material at edges of the channel, called the 'lip', after multiple passes of the laser. The line of best fit, its equation and $R^{2}$ are shown. 


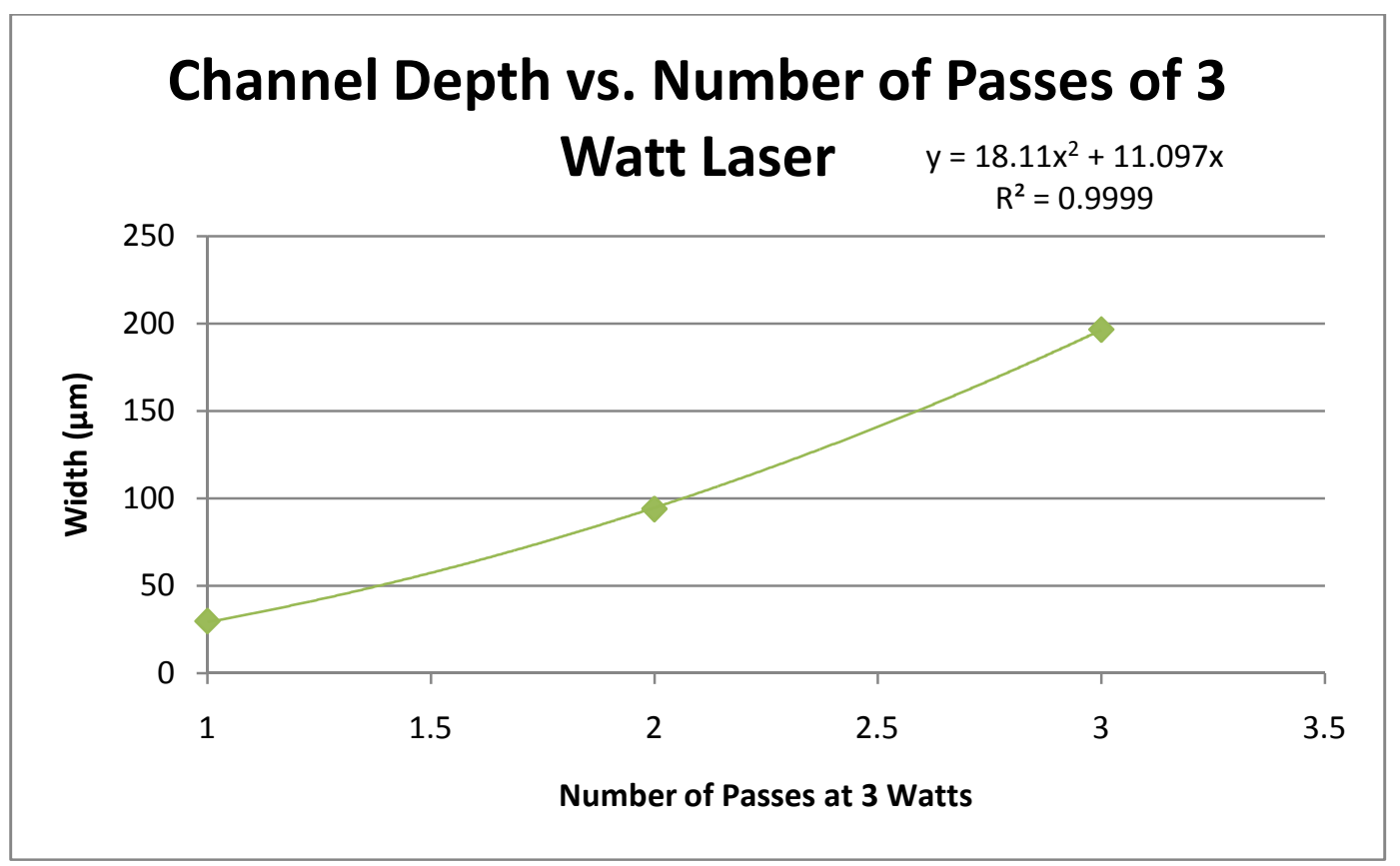

Figure 99 - Maximum depth of channel versus number of laser passes 3 watt $\mathrm{CO}_{2}$. The line was modeled with a logarithmic fit based on the data from literature. The equation and $R^{2}$ value are shown.

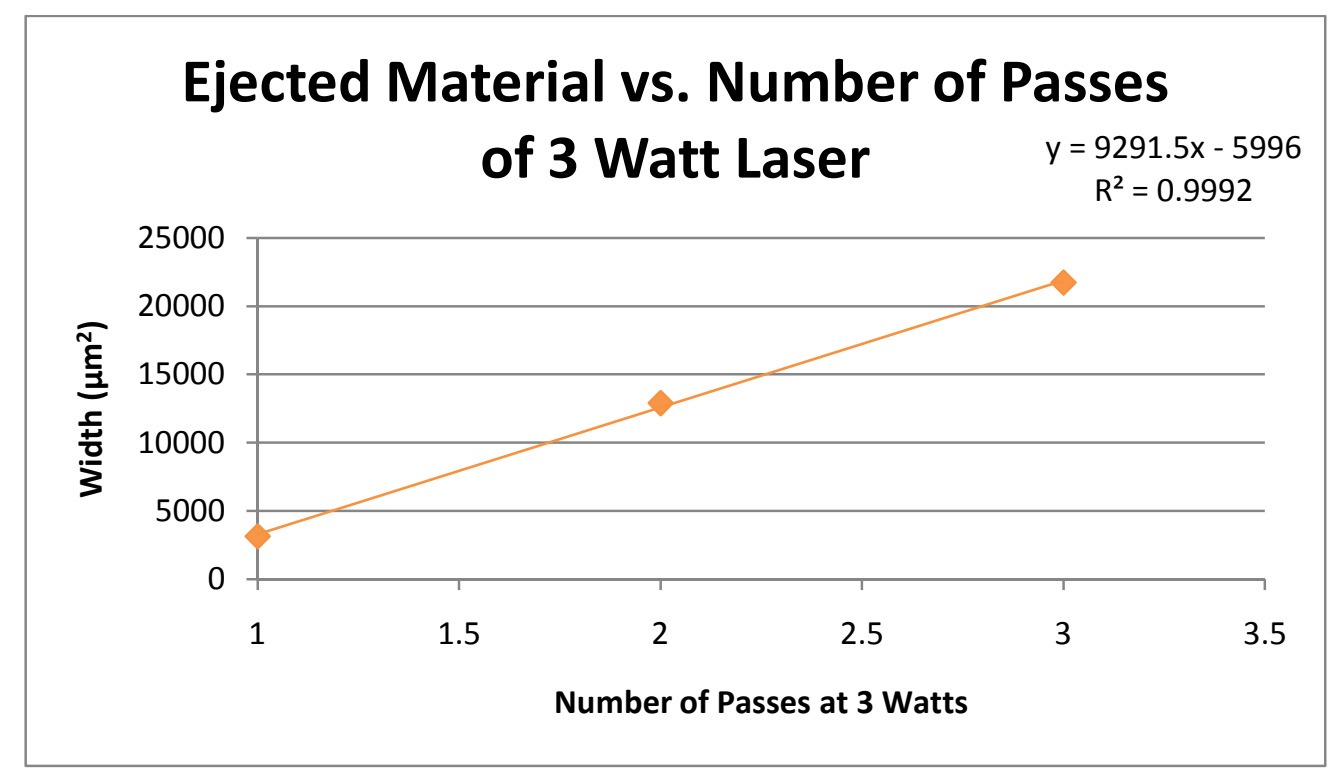

Figure 100 - Material ejected from the channel versus number of laser passes with a 3 watt $\mathrm{CO}_{2}$ laser. The values were found by integrating with a left Riemann sum. 


\subsubsection{Comparison of Different Laser Powers Used in Raster Etching}

Raster cuts were done in order determine the laser's effectiveness at creating a chamber of consistent depth, something that is well established in PDMS microfabrication but not as much in PMMA laser microfrabrication. The 5 watt data became corrupted during analysis so it is not shown.

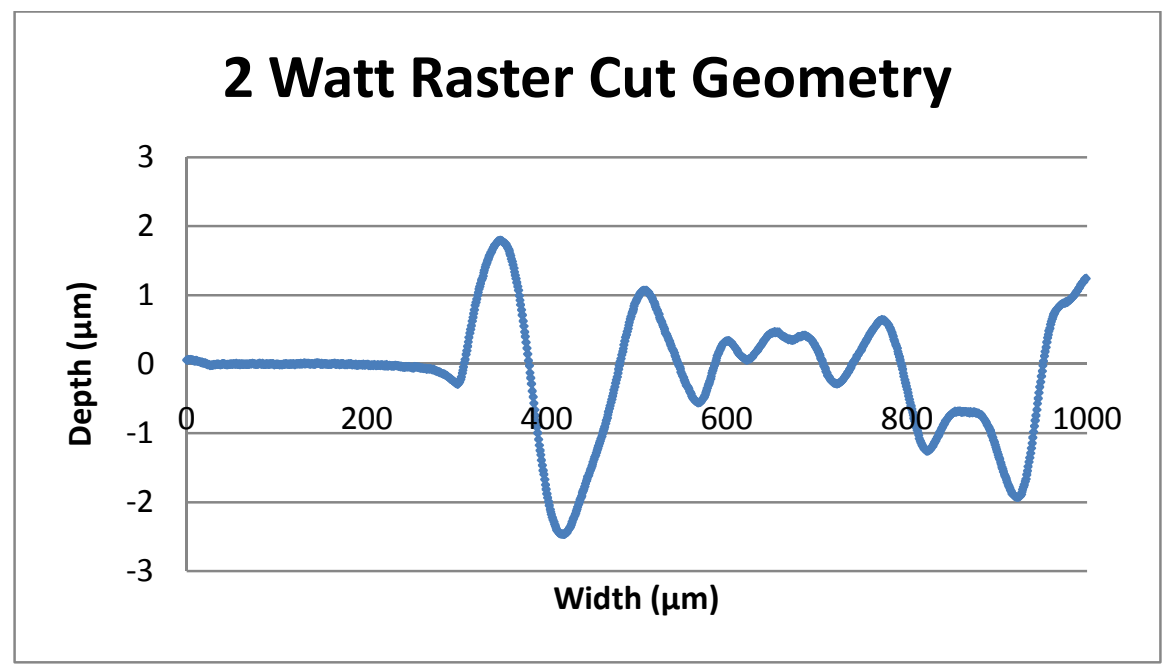

Figure 101 - Geometry of a raster cut created with a 2 watt $\mathrm{CO}_{2}$ laser. The 0 line of the $\mathrm{Y}$-axis at the left of the graph indicates the baseline of the PMMA before reaching material that had been etched by the laser

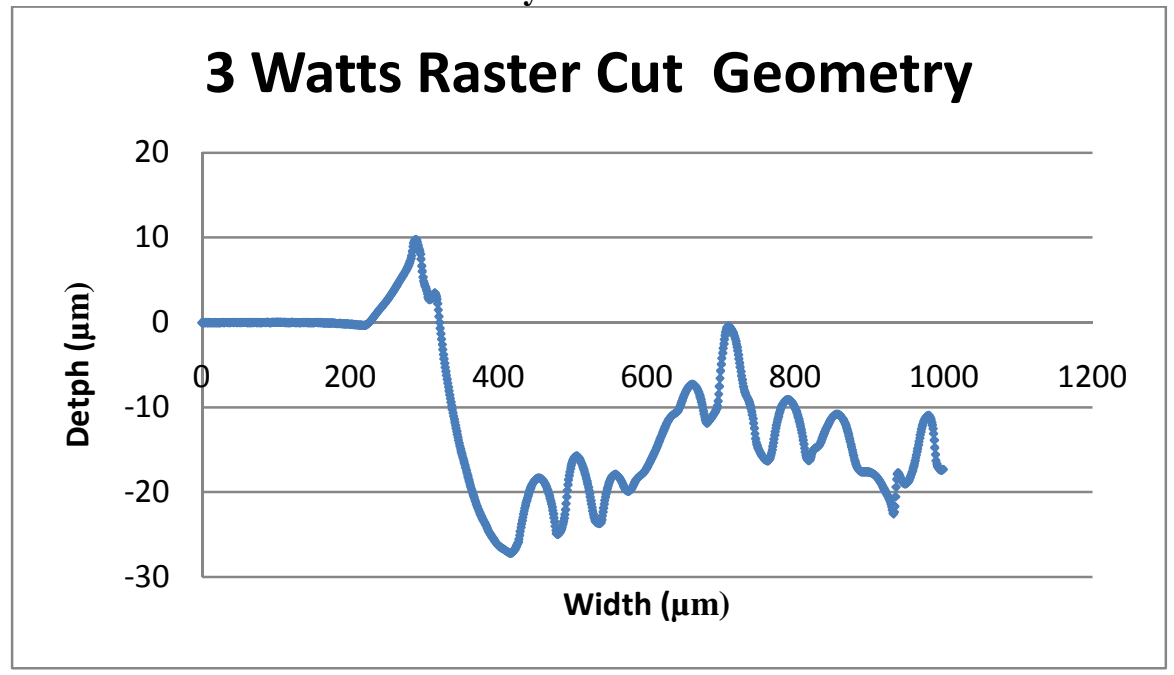

Figure 102 - Geometry of a raster cut created with a 3 watt $\mathrm{CO}_{2}$ laser. The 0 line of the $\mathrm{Y}$-axis at the left of the graph indicates the baseline of the PMMA before reaching material that had been etched by the laser 


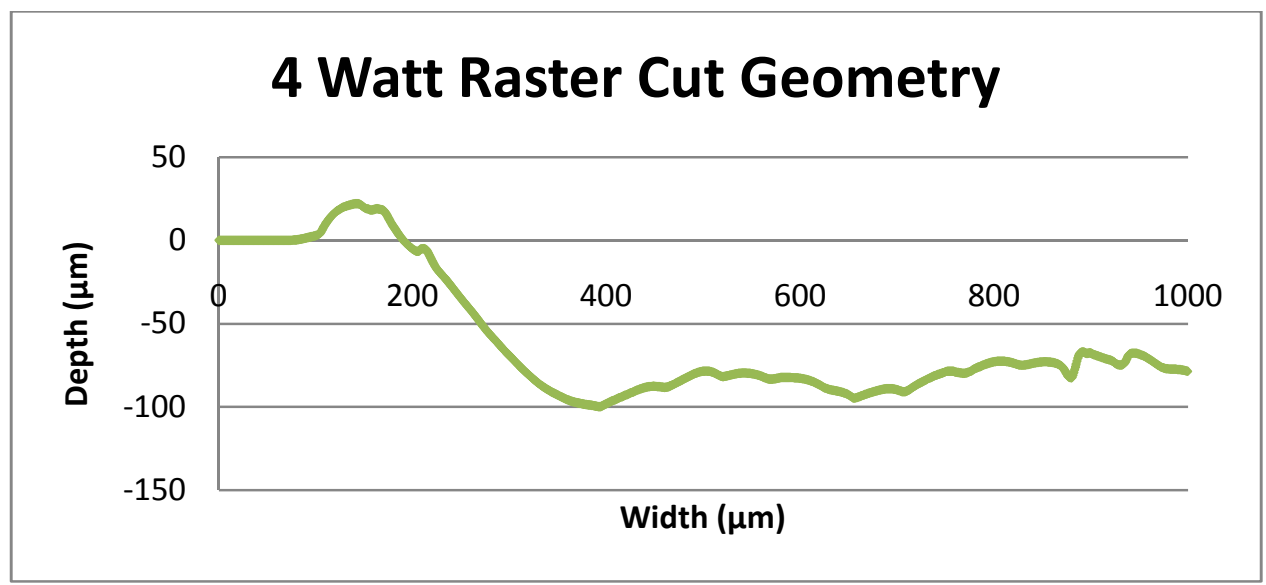

Figure 103 - Geometry of a raster cut created with a 4 watt $\mathrm{CO}_{2}$ laser. The 0 line of the $\mathrm{Y}$-axis at the left of the graph indicates the baseline of the PMMA before reaching material that had been etched by the laser

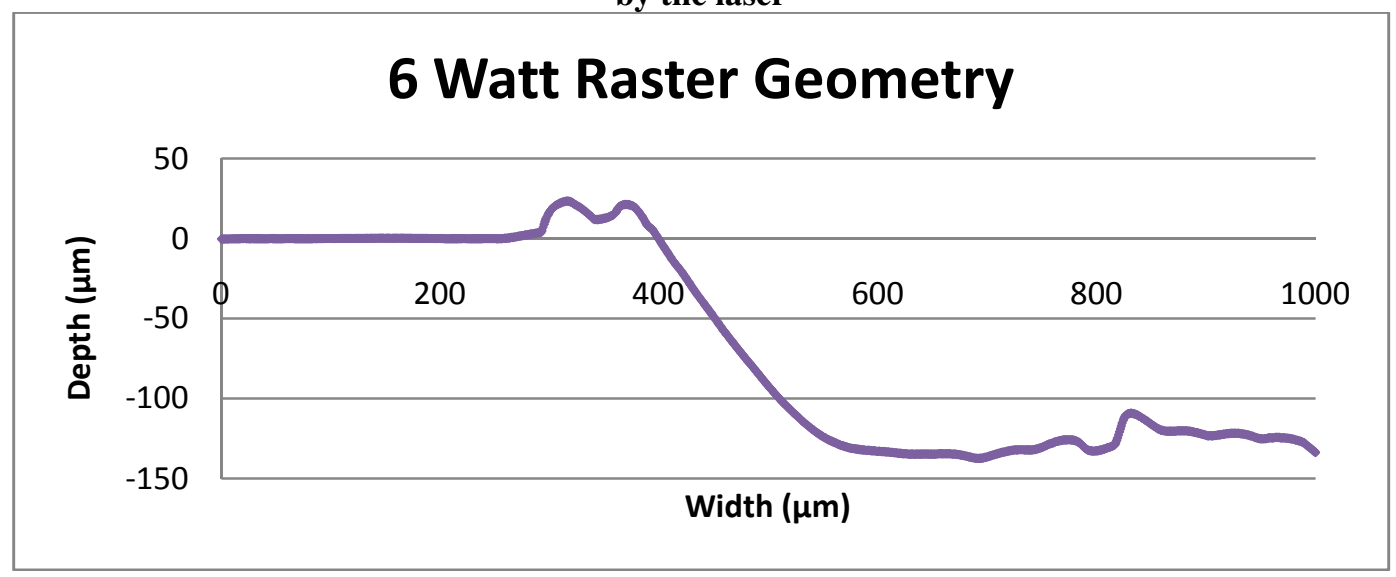

Figure 104 - Geometry of a raster cut created with a 5 watt $\mathrm{CO}_{2}$ laser. The 0 line of the $\mathrm{Y}$-axis at the left of the graph indicates the baseline of the PMMA before reaching material that had been etched by the laser 


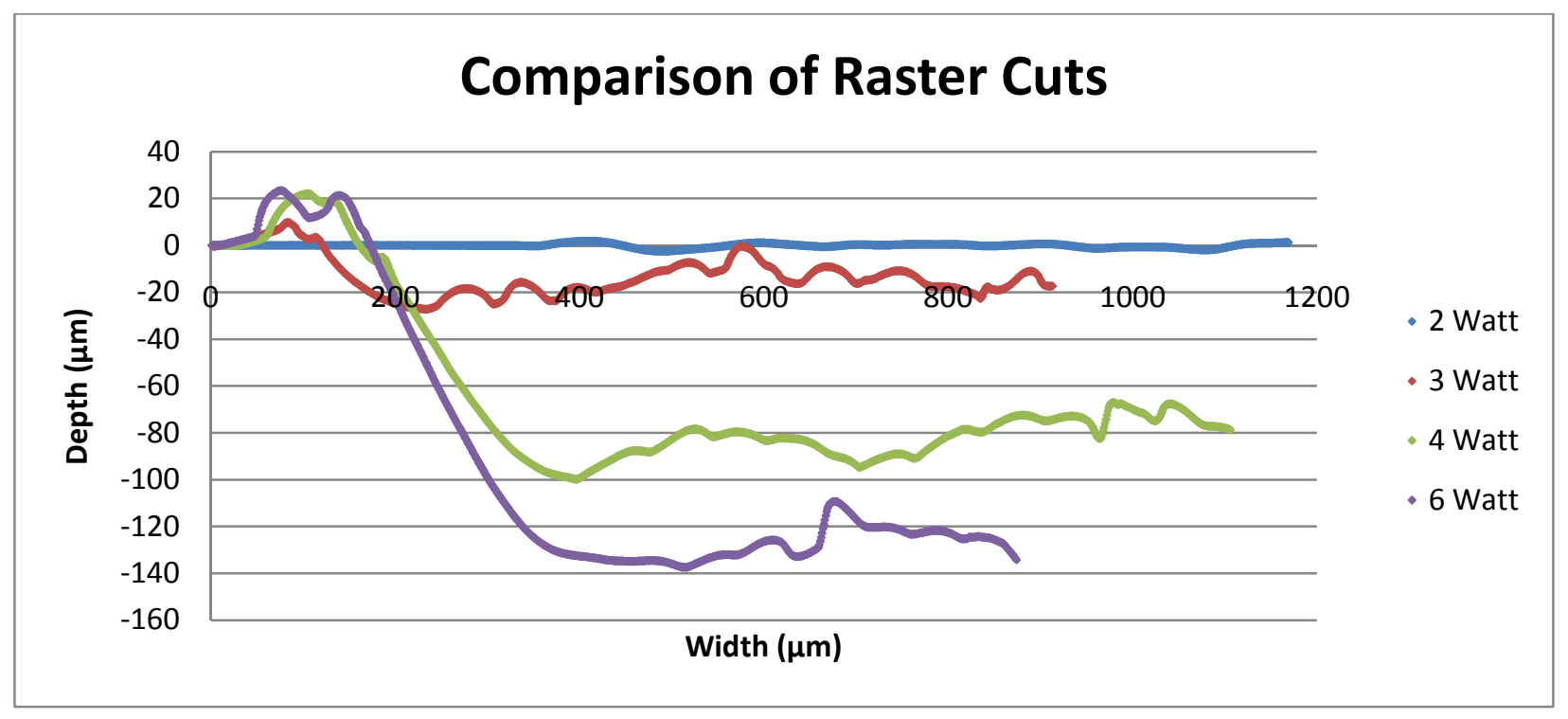

Figure 105 - Overlaid comparison of raster etching powers of a $\mathrm{CO}_{2}$ laser. The 0 line of the $\mathrm{Y}$-axis at the left of the graph indicates the baseline of the PMMA before reaching material that had been etched by the laser

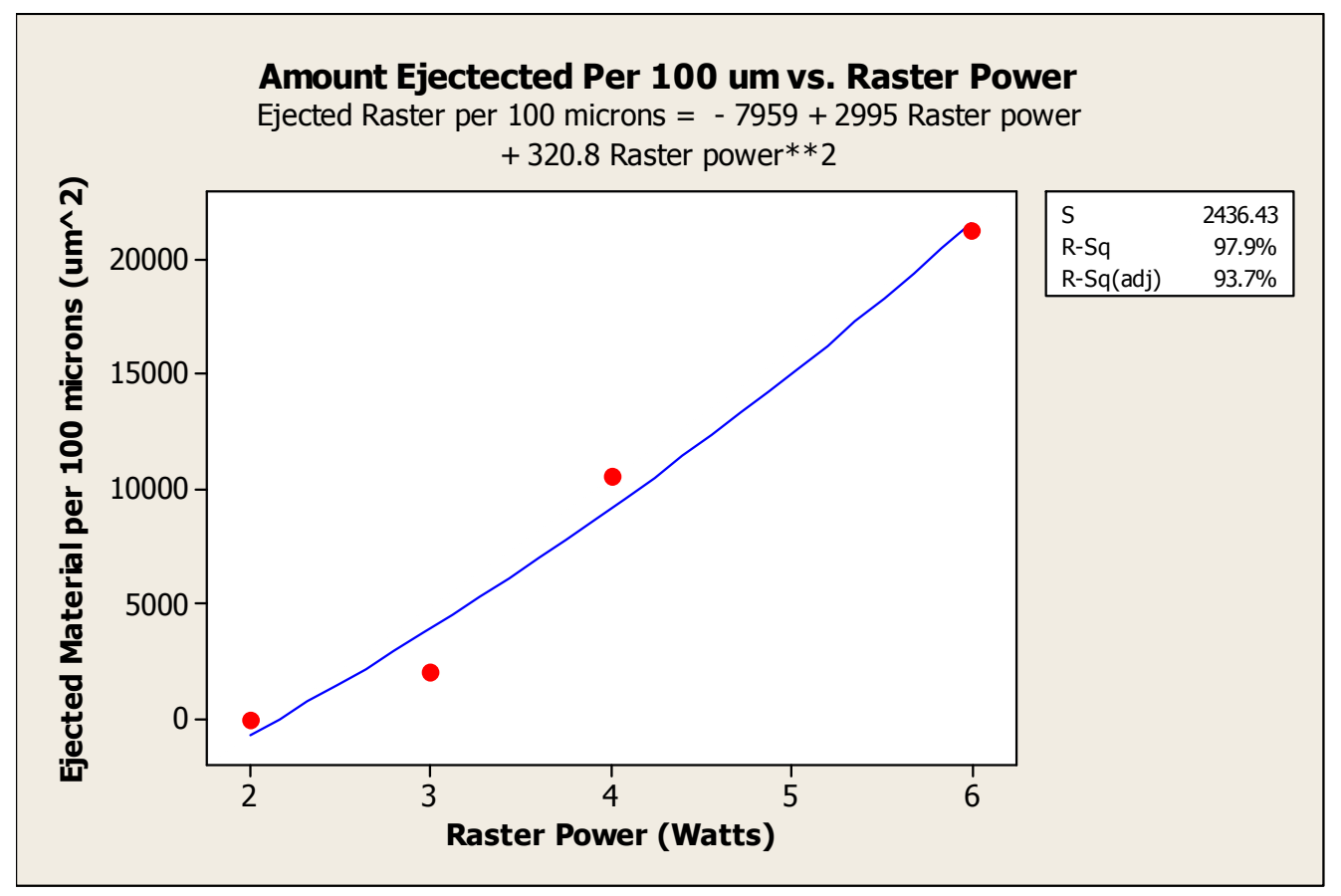

Figure 106 - Scatterplot of amount of material ejected from rastering process per $100 \mu \mathrm{m}$ of width. The values were found by integrating with a left Riemann sum starting from the lowest point of the graph, the center of the beam of the first pass. 


\subsubsection{Comparison of Channel Roughness versus Different Factors}

Channel roughness was found by starting the profilometer data recording with the probe already in the channel. The channels were lined up as well as possible with the path of the profilometer but linear trends inevitably arose. The upward or downward trend in the data was noted and a line was drawn from the beginning of the data to the end. The points from this line were subtracted from the $y$-component of the data to give level roughness lines for easier visual comparison. Note that in this section the axes are not scaled to facilitate easier viewing of the micro-scale roughness.

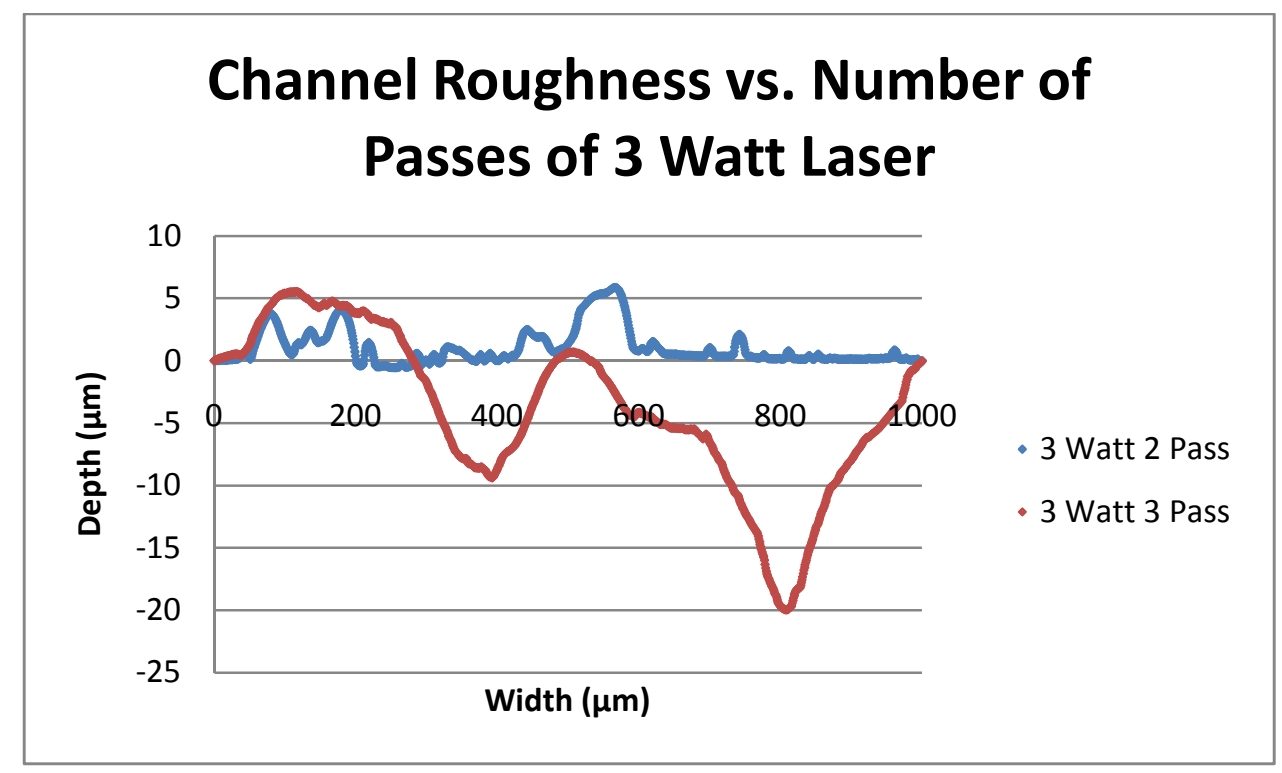

Figure 107 - Roughness along channels with multiple passes of a 3 watt laser. 


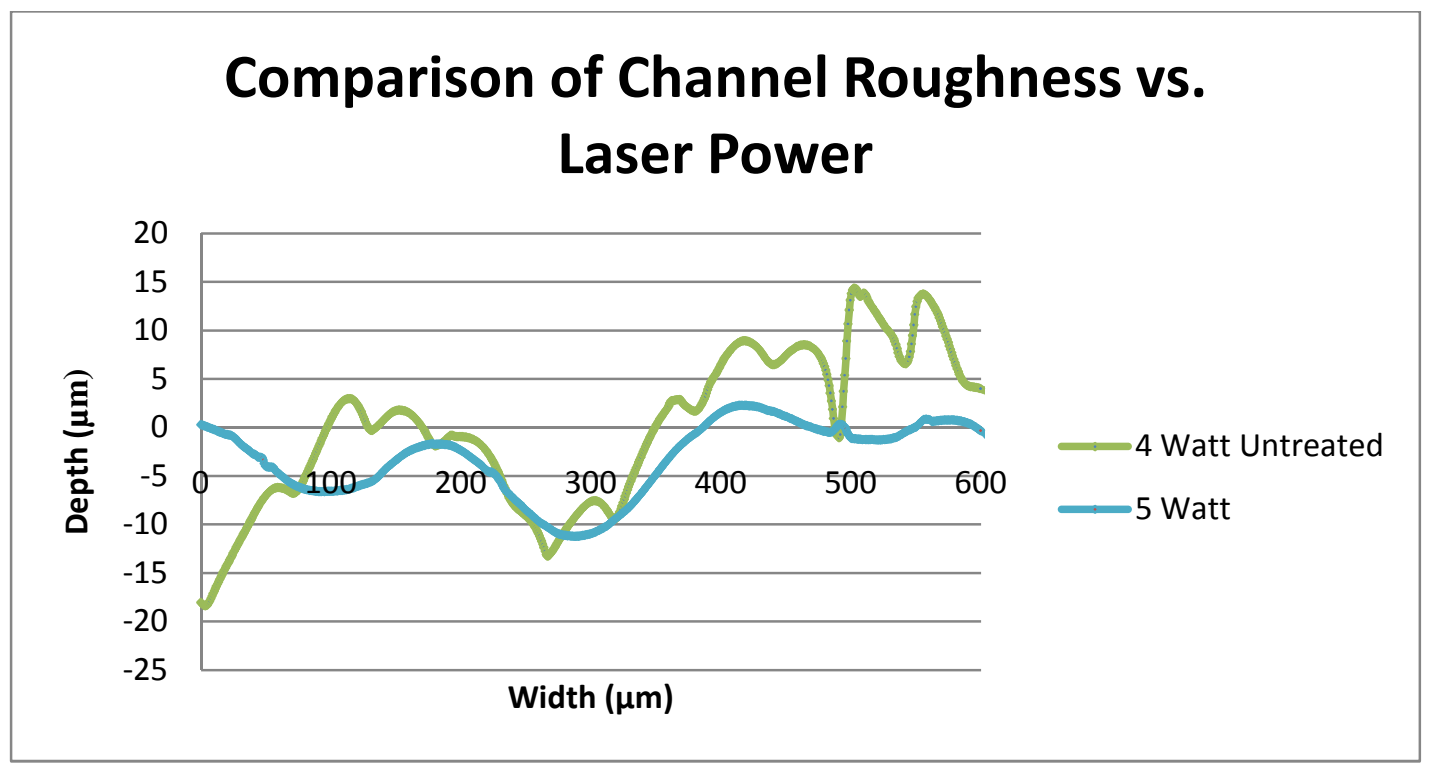

Figure 108 - Comparison of channel roughness versus 4 and 5 watt $\mathrm{CO}_{2}$ laser power

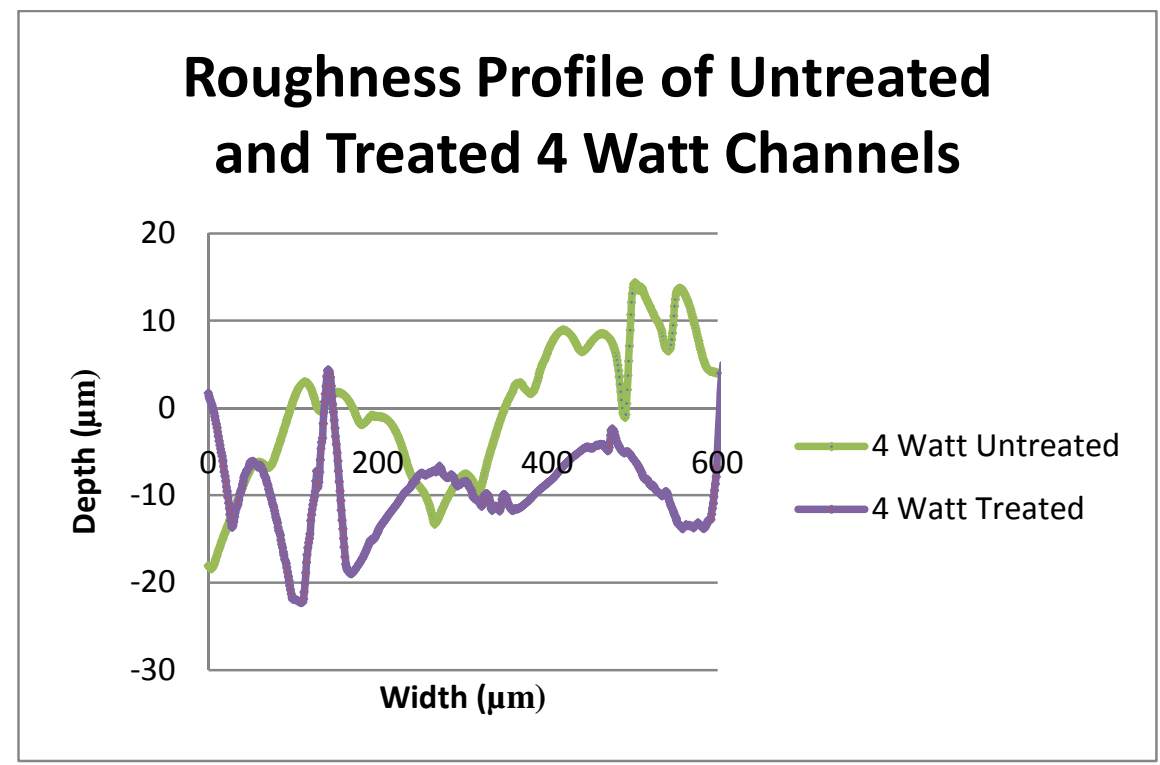

Figure 109 - Comparison of effects of heat treatment versus no treatment on roughness of channels created with the same 4 watt $\mathrm{CO}_{2}$ laser power 


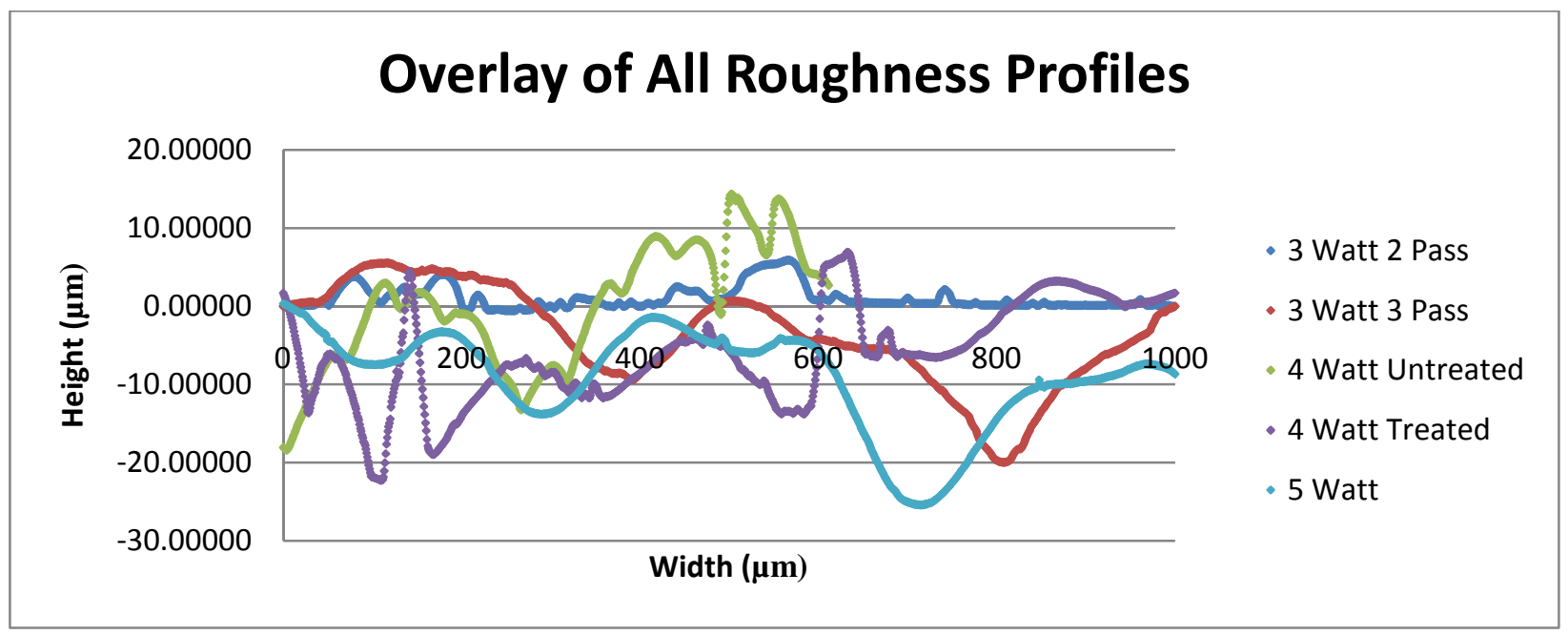

Figure 110 - Overlay graph of all methods of fabrication used for laser etched channels

\subsection{Statistical Analysis of Sphere Velocity Data}

Electrophoresis experiment raw data is not listed in this section; instead, graphs of the analysis of this data are listed. Analysis of the data was deemed to be of more interest to the readers of this thesis than raw data. Comparing averages of data to one another is an insufficient method of analysis for a thesis. A t-test was instead used to compare the data. This statistical analysis method factors in the means, averages and standard deviations of the data sets. For all tests, a 95\% confidence interval was used. Cell shading was used to indicate passed or failed tests with the criteria explained in the figure's comment. 
Table 3 - Chart listing the results of the T-test comparison of 1 and 5 micron particles at different voltages and locations. A 95\% confidence level was used. The $P \neq$ column is a simple T-test to show if the results are not equal and the $P<$ column values are the results testing if the 1 micron particles move slower. The cells highlighted green agree with the hypothesis ( 1 and 5 micron particles give different results) and the cells highlighted red disagree with the hypothesis.

\begin{tabular}{|l|r|r|}
\hline & $\mathrm{P} \neq$ & $\mathrm{P}<$ \\
\hline $\begin{array}{l}\text { 1 vs. 5 } \\
\text { micron }\end{array}$ & & \\
\hline Beginning & & \\
\hline $500 \mathrm{~V}$ & 0.000 & 0.000 \\
\hline $1000 \mathrm{~V}$ & 0.000 & 0.000 \\
\hline $1500 \mathrm{~V}$ & 0.609 & 0.305 \\
\hline Middle & & \\
\hline $500 \mathrm{~V}$ & 0.053 & 0.027 \\
\hline $1000 \mathrm{~V}$ & 0.003 & 0.002 \\
\hline $1500 \mathrm{~V}$ & 0.000 & 0.000 \\
\hline End & & \\
\hline $500 \mathrm{~V}$ & 0.170 & 0.085 \\
\hline $1000 \mathrm{~V}$ & 0.215 & 0.108 \\
\hline $1500 \mathrm{~V}$ & 0.033 & 0.017 \\
\hline
\end{tabular}

Table 4 - Chart listing the results of the T-test comparison of 5 and 10 micron particles at different voltages and locations. A 95\% confidence level was used. The $P \neq$ column is a simple T-test to show if the results are not equal and the $P<$ column values are the results testing if the 5 micron particles move slower. The cells highlighted green agree with the hypothesis ( 5 and 10 micron particles give different results) and the cells highlighted red disagree with the hypothesis.

\begin{tabular}{|l|l|l|}
\hline & $\mathrm{P} \neq$ & $\mathrm{P}<$ \\
\hline $\begin{array}{l}\text { 5 vs. 10 } \\
\text { micron }\end{array}$ & & \\
\hline Beginning & & \\
\hline $500 \mathrm{~V}$ & 0.000 & 0.000 \\
\hline $1000 \mathrm{~V}$ & 0.000 & 0.000 \\
\hline $1500 \mathrm{~V}$ & 0.000 & 0.000 \\
\hline $2000 \mathrm{~V}$ & 0.000 & 0.000 \\
\hline Middle & & \\
\hline $500 \mathrm{~V}$ & 0.000 & 0.000 \\
\hline $1000 \mathrm{~V}$ & 0.000 & 0.000 \\
\hline $1500 \mathrm{~V}$ & 0.042 & 0.021 \\
\hline $2000 \mathrm{~V}$ & 0.018 & 0.009 \\
\hline End & & \\
\hline $500 \mathrm{~V}$ & 0.156 & 0.078 \\
\hline $1000 \mathrm{~V}$ & 0.116 & 0.058 \\
\hline $1500 \mathrm{~V}$ & 0.084 & 0.042 \\
\hline $2000 \mathrm{~V}$ & 0.256 & 0.128 \\
\hline
\end{tabular}


Table 5 - Chart listing the results of the T-test comparison of 1 and 10 micron particles at different voltages and locations. A 95\% confidence level was used. The $\mathbf{P} \neq$ column is a simple $T$-test to show if the results are not equal and the $P<$ column values are the results testing if the 5 micron particles move slower. The cells highlighted green agree with the hypothesis (1 and 10 micron particles give different results) and the cells highlighted red disagree with the hypothesis.

\begin{tabular}{|l|r|r|}
\hline & $\mathrm{P} \neq$ & $\mathrm{P}<$ \\
\hline $\begin{array}{l}\text { 1 vs. 10 } \\
\text { micron }\end{array}$ & & \\
\hline Beginning & & \\
\hline $500 \mathrm{~V}$ & 0.000 & 0.000 \\
\hline $1000 \mathrm{~V}$ & 0.000 & 0.000 \\
\hline $1500 \mathrm{~V}$ & 0.000 & 0.000 \\
\hline Middle & & \\
\hline $500 \mathrm{~V}$ & 0.000 & 0.000 \\
\hline $1000 \mathrm{~V}$ & 0.000 & 0.000 \\
\hline $1500 \mathrm{~V}$ & 0.003 & 0.002 \\
\hline End & & \\
\hline $500 \mathrm{~V}$ & 0.000 & 0.000 \\
\hline $1000 \mathrm{~V}$ & 0.000 & 0.000 \\
\hline $1500 \mathrm{~V}$ & 0.071 & 0.036 \\
\hline
\end{tabular}

Table 6 - Chart listing the results of the T-test comparison of 1 micron particles the same voltage at different locations. A 95\% confidence level was used. The T-test was used to see if 1 micron particles move at similar speeds at different sections of the channel. The cells highlighted green agree with the hypothesis (1 micron particles move at similar speeds regardless of location) and the cells highlighted red disagree with the hypothesis.

\begin{tabular}{|l|l|}
\hline 1 micron & $\mathrm{P}+$ \\
\hline $\begin{array}{l}500 \mathrm{~V} \text { beg vs. } \\
\text { mid }\end{array}$ & 0.001 \\
\hline $\begin{array}{l}500 \mathrm{~V} \text { mid vs. } \\
\text { end }\end{array}$ & 0.000 \\
\hline $\begin{array}{l}500 \mathrm{~V} \text { beg vs. } \\
\text { end }\end{array}$ & 0.032 \\
\hline $\begin{array}{l}1000 \mathrm{~V} \text { beg vs. } \\
\text { mid }\end{array}$ & 0.000 \\
\hline $\begin{array}{l}1000 \mathrm{v} \text { mid vs. } \\
\text { end }\end{array}$ & 0.873 \\
\hline $\begin{array}{l}1000 \mathrm{~V} \text { beg vs. } \\
\text { end }\end{array}$ & 0.123 \\
\hline $\begin{array}{l}1500 \mathrm{~V} \text { beg vs. } \\
\text { mid }\end{array}$ & 0.360 \\
\hline $\begin{array}{l}1500 \mathrm{~V} \text { mid vs. } \\
\text { end }\end{array}$ & 0.169 \\
\hline $\begin{array}{l}1500 \mathrm{~V} \text { beg vs. } \\
\text { end }\end{array}$ & \\
\hline
\end{tabular}


Table 7 - Chart listing the results of the T-test comparison of 5 micron particles the same voltage at different locations. A 95\% confidence level was used. The T-test was used to see if 5 micron particles move at similar speeds at different sections of the channel. The cells highlighted green agree with the hypothesis ( 5 micron particles move at similar speeds regardless of location) and the cells highlighted red disagree with the hypothesis

\begin{tabular}{|l|c|}
\hline 5 micron & \\
\hline $\begin{array}{l}500 \mathrm{~V} \text { beg vs. } \\
\text { mid }\end{array}$ & 0.531 \\
\hline $\begin{array}{l}500 \mathrm{~V} \text { mid vs. } \\
\text { end }\end{array}$ & 0.464 \\
\hline $\begin{array}{l}500 \mathrm{~V} \text { beg vs. } \\
\text { end }\end{array}$ & 0.366 \\
\hline $\begin{array}{l}1000 \mathrm{~V} \text { beg vs. } \\
\text { mid }\end{array}$ & 0.000 \\
\hline $\begin{array}{l}1000 \mathrm{v} \text { mid vs. } \\
\text { end }\end{array}$ & 0.260 \\
\hline $\begin{array}{l}1000 \mathrm{~V} \text { beg vs. } \\
\text { end }\end{array}$ & 0.000 \\
\hline $\begin{array}{l}1500 \mathrm{~V} \text { beg vs. } \\
\text { mid }\end{array}$ & 0.000 \\
\hline $\begin{array}{l}1500 \mathrm{~V} \text { mid vs. } \\
\text { end }\end{array}$ & 0.043 \\
\hline $\begin{array}{l}1500 \mathrm{~V} \text { beg vs. } \\
\text { end }\end{array}$ & 0.029 \\
\hline $\begin{array}{l}\text { 2000V beg vs. } \\
\text { mid }\end{array}$ & 0.190 \\
\hline $\begin{array}{l}\text { 2000V mid vs. } \\
\text { end }\end{array}$ & 0.008 \\
\hline $\begin{array}{l}\text { 2000V beg vs. } \\
\text { end }\end{array}$ & \\
\hline
\end{tabular}


Table 8 - Chart listing the results of the T-test comparison of 10 micron particles the same voltage at different locations. A $95 \%$ confidence level was used. The T-test was used to see if 10 micron particles move at similar speeds at different sections of the channel. The cells highlighted green agree with the hypothesis (10 micron particles move at similar speeds regardless of location) and the cells highlighted red disagree with the hypothesis.

\begin{tabular}{|l|l|}
\hline 10 micron & \\
\hline $\begin{array}{l}500 \mathrm{~V} \text { beg vs. } \\
\text { mid }\end{array}$ & 0.026 \\
\hline $\begin{array}{l}500 \mathrm{~V} \text { mid vs. } \\
\text { end }\end{array}$ & 0.142 \\
\hline $\begin{array}{l}500 \mathrm{~V} \text { beg vs. } \\
\text { end }\end{array}$ & 0.082 \\
\hline $\begin{array}{l}\text { 1000V beg vs. } \\
\text { mid }\end{array}$ & 0.234 \\
\hline $\begin{array}{l}1000 \mathrm{v} \text { mid vs. } \\
\text { end }\end{array}$ & 0.000 \\
\hline $\begin{array}{l}1000 \mathrm{~V} \text { beg vs. } \\
\text { end }\end{array}$ & 0.043 \\
\hline $\begin{array}{l}1500 \mathrm{~V} \text { beg vs. } \\
\text { mid }\end{array}$ & 0.001 \\
\hline $\begin{array}{l}\text { 1500V mid vs. } \\
\text { end }\end{array}$ & 0.003 \\
\hline $\begin{array}{l}1500 \mathrm{~V} \text { beg vs. } \\
\text { end }\end{array}$ & 0.023 \\
\hline $\begin{array}{l}\text { 2000V beg vs. } \\
\text { mid }\end{array}$ & 0.399 \\
\hline $\begin{array}{l}\text { 2000V mid vs. } \\
\text { end }\end{array}$ & 0.122 \\
\hline $\begin{array}{l}\text { 2000V beg vs. } \\
\text { end }\end{array}$ & 0.157 \\
\hline
\end{tabular}

Table 9 - Chart comparing 1 micron particle movement at the same location while under the influence of different voltages. The T-test was used to see if the particles move at different speeds based on voltage. A $95 \%$ confidence level was used. The $P \neq$ column tests the hypothesis that the speeds of the particles at two different voltages are not the same and the $P<$ column values are the results of the hypothesis that the higher voltages elicit greater speeds. The cells highlighted green agree with the respective hypothesis and the ones highlighted red disagree.

\begin{tabular}{|c|c|c|c|c|c|c|c|c|}
\hline 1 micron & & & & & & & & \\
\hline Beginning & $\mathrm{P} \neq$ & $\mathrm{P}<$ & Middle & $\mathrm{P} \neq$ & $\mathrm{P}<$ & End & $\mathrm{P} \neq$ & $\mathrm{P}<$ \\
\hline $\begin{array}{l}500 \text { vs. } \\
1000\end{array}$ & 0.020 & 0.010 & $\begin{array}{l}500 \text { vs. } \\
1000\end{array}$ & 0.045 & 0.023 & $\begin{array}{l}500 \text { vs. } \\
1000\end{array}$ & 0.958 & 0.479 \\
\hline $\begin{array}{l}500 \text { vs. } \\
1500\end{array}$ & 0.000 & 0.000 & $\begin{array}{l}500 \text { vs. } \\
1500\end{array}$ & 0.000 & 0.000 & $\begin{array}{l}500 \text { vs. } \\
1500\end{array}$ & 0.031 & 0.016 \\
\hline $\begin{array}{l}1000 \text { vs. } \\
1500\end{array}$ & 0.000 & 0.000 & $\begin{array}{l}1000 \text { vs. } \\
1500\end{array}$ & 0.000 & 0.000 & $\begin{array}{l}1000 \text { vs. } \\
1500\end{array}$ & 0.026 & 0.013 \\
\hline
\end{tabular}


Table 10 - Chart comparing 5 micron particle movement at the same location while under the influence of different voltages. The T-test was used to see if the particles move at different speeds based on voltage. A $95 \%$ confidence level was used. The $\mathbf{P} \neq$ column tests the hypothesis that the speeds of the particles at two different voltages are not the same and the $\mathrm{P}<$ column values are the results of the hypothesis that the higher voltages elicit greater speeds. The cells highlighted green agree with the respective hypothesis and the ones highlighted red disagree

\begin{tabular}{|c|c|c|c|c|c|c|c|c|}
\hline Beginning & $\mathrm{P} \neq$ & $\mathrm{P}<$ & Middle & $\mathrm{P} \neq$ & $\mathrm{P}<$ & End & $\mathrm{P} \neq$ & $\mathrm{P}<$ \\
\hline $\begin{array}{l}500 \text { vs. } \\
1000\end{array}$ & 0.000 & 0.000 & $\begin{array}{l}500 \text { vs. } \\
1000\end{array}$ & 0.000 & 0.000 & $\begin{array}{l}500 \text { vs. } \\
1000\end{array}$ & 0.045 & 0.023 \\
\hline $\begin{array}{l}500 \text { vs. } \\
1500\end{array}$ & 0.000 & 0.000 & $\begin{array}{l}500 \text { vs. } \\
1500\end{array}$ & 0.000 & 0.000 & $\begin{array}{l}500 \text { vs. } \\
1500\end{array}$ & 0.009 & 0.005 \\
\hline $\begin{array}{l}500 \text { vs. } \\
2000\end{array}$ & 0.000 & 0.000 & $\begin{array}{l}500 \text { vs. } \\
2000\end{array}$ & 0.000 & 0.000 & $\begin{array}{l}500 \text { vs. } \\
2000\end{array}$ & 0.000 & 0.000 \\
\hline $\begin{array}{l}1000 \text { vs. } \\
1500\end{array}$ & 0.000 & 0.000 & $\begin{array}{l}1000 \text { vs. } \\
1500 \text {. }\end{array}$ & 0.000 & 0.000 & $\begin{array}{l}1000 \text { vs. } \\
1500\end{array}$ & 0.012 & 0.006 \\
\hline $\begin{array}{l}1000 \text { vs. } \\
2000\end{array}$ & 0.000 & 0.000 & $\begin{array}{l}1000 \text { vs. } \\
2000\end{array}$ & 0.000 & 0.000 & $\begin{array}{l}1000 \text { vs. } \\
2000\end{array}$ & 0.000 & 0.000 \\
\hline $\begin{array}{l}1500 \text { vs. } \\
2000\end{array}$ & 0.000 & 0.000 & $\begin{array}{l}1500 \text { vs. } \\
2000\end{array}$ & 0.002 & 0.001 & $\begin{array}{l}1500 \text { vs. } \\
2000\end{array}$ & 0.000 & 0.000 \\
\hline
\end{tabular}

Table 11 - Chart comparing 10 micron particle movement at the same location while under the influence of different voltages. The T-test was used to see if the particles move at different speeds based on voltage. A $95 \%$ confidence level was used. The $P \neq$ column tests the hypothesis that the speeds of the particles at two different voltages are not the same and the $P<$ column values are the results of the hypothesis that the higher voltages elicit greater speeds. The cells highlighted green agree with the respective hypothesis and the ones highlighted red disagree.

\begin{tabular}{|c|c|c|c|c|c|c|c|c|}
\hline 10 micron & $\mathrm{P} \neq$ & $\mathrm{P}<$ & & & & & & \\
\hline Beginning & $\mathrm{P} \neq$ & $\mathrm{P}<$ & Middle & $\mathrm{P} \neq$ & $\mathrm{P}<$ & End & $\mathrm{P} \neq$ & $\mathrm{P}<$ \\
\hline $\begin{array}{l}500 \text { vs. } \\
1000\end{array}$ & 0.116 & 0.058 & $\begin{array}{l}500 \text { vs. } \\
1000\end{array}$ & 0.001 & 0.001 & $\begin{array}{l}500 \text { vs. } \\
1000\end{array}$ & 0.017 & 0.009 \\
\hline $\begin{array}{l}500 \text { vs. } \\
1500\end{array}$ & 0.032 & 0.016 & $\begin{array}{l}500 \text { vs. } \\
1500\end{array}$ & 0.000 & 0.000 & $\begin{array}{l}500 \text { vs. } \\
1500\end{array}$ & 0.010 & 0.005 \\
\hline $\begin{array}{l}500 \text { vs. } \\
2000\end{array}$ & 0.000 & 0.000 & $\begin{array}{l}500 \text { vs. } \\
2000\end{array}$ & 0.033 & 0.017 & $\begin{array}{l}500 \text { vs. } \\
2000\end{array}$ & 0.008 & 0.004 \\
\hline $\begin{array}{l}1000 \text { vs. } \\
1500\end{array}$ & 0.047 & 0.024 & $\begin{array}{l}1000 \text { vs. } \\
1500\end{array}$ & 0.001 & 0.001 & $\begin{array}{l}1000 \text { vs. } \\
1500\end{array}$ & 0.018 & 0.009 \\
\hline $\begin{array}{l}1000 \text { vs. } \\
2000\end{array}$ & 0.035 & 0.018 & $\begin{array}{l}1000 \text { vs. } \\
2000\end{array}$ & 0.035 & 0.018 & $\begin{array}{l}1000 \text { vs. } \\
2000\end{array}$ & 0.009 & 0.005 \\
\hline $\begin{array}{l}1500 \text { vs. } \\
2000\end{array}$ & 0.000 & 0.000 & $\begin{array}{l}1500 \text { vs. } \\
2000\end{array}$ & 0.560 & 0.280 & $\begin{array}{l}1500 \text { vs. } \\
2000\end{array}$ & 0.015 & 0.008 \\
\hline
\end{tabular}




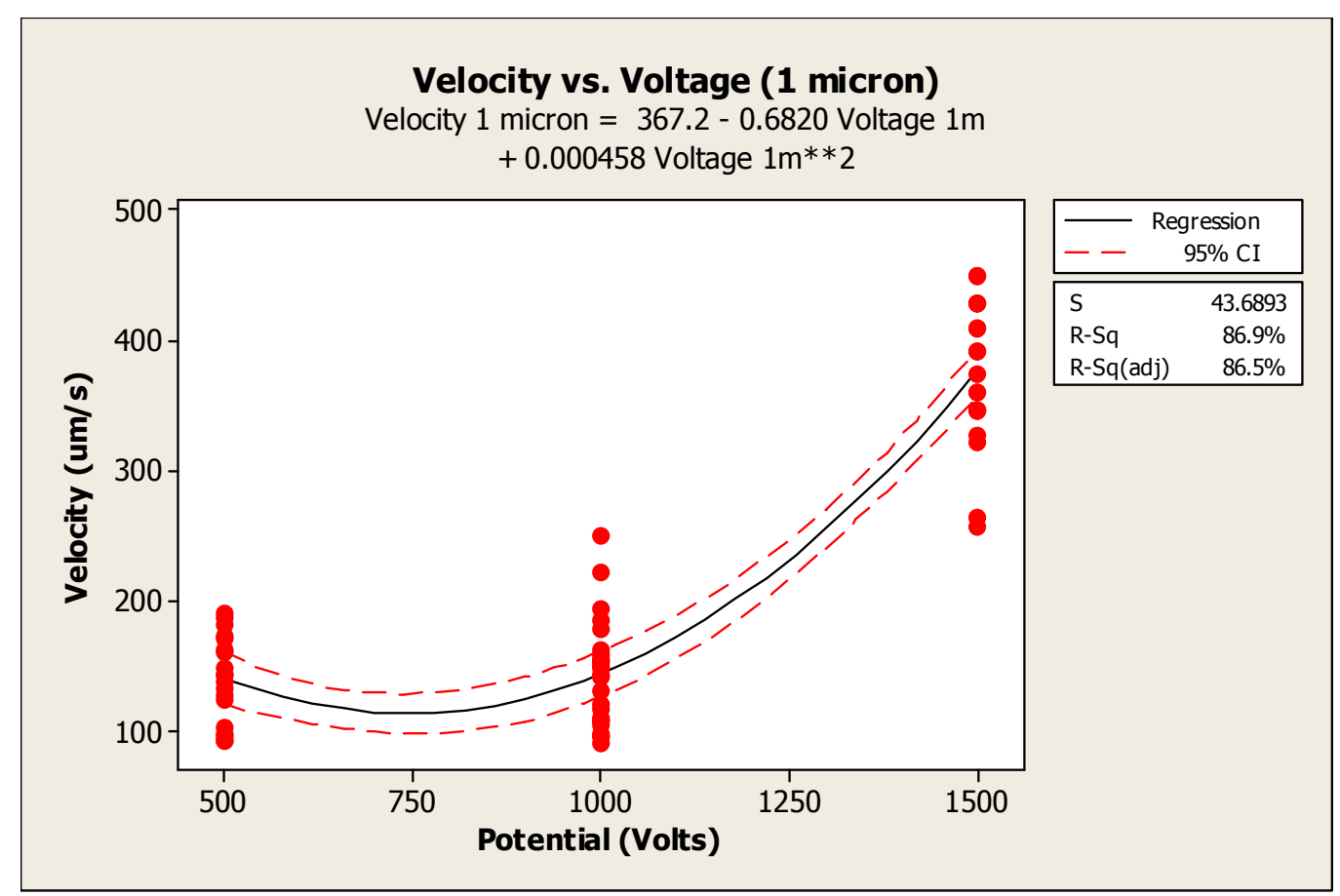

Figure 111 - Scatterplot of microsphere velocity versus voltage of 1 micron particles with polynomial line of best fit and $95 \%$ confidence interval. $\mathbf{R}^{2}$ values are also shown.

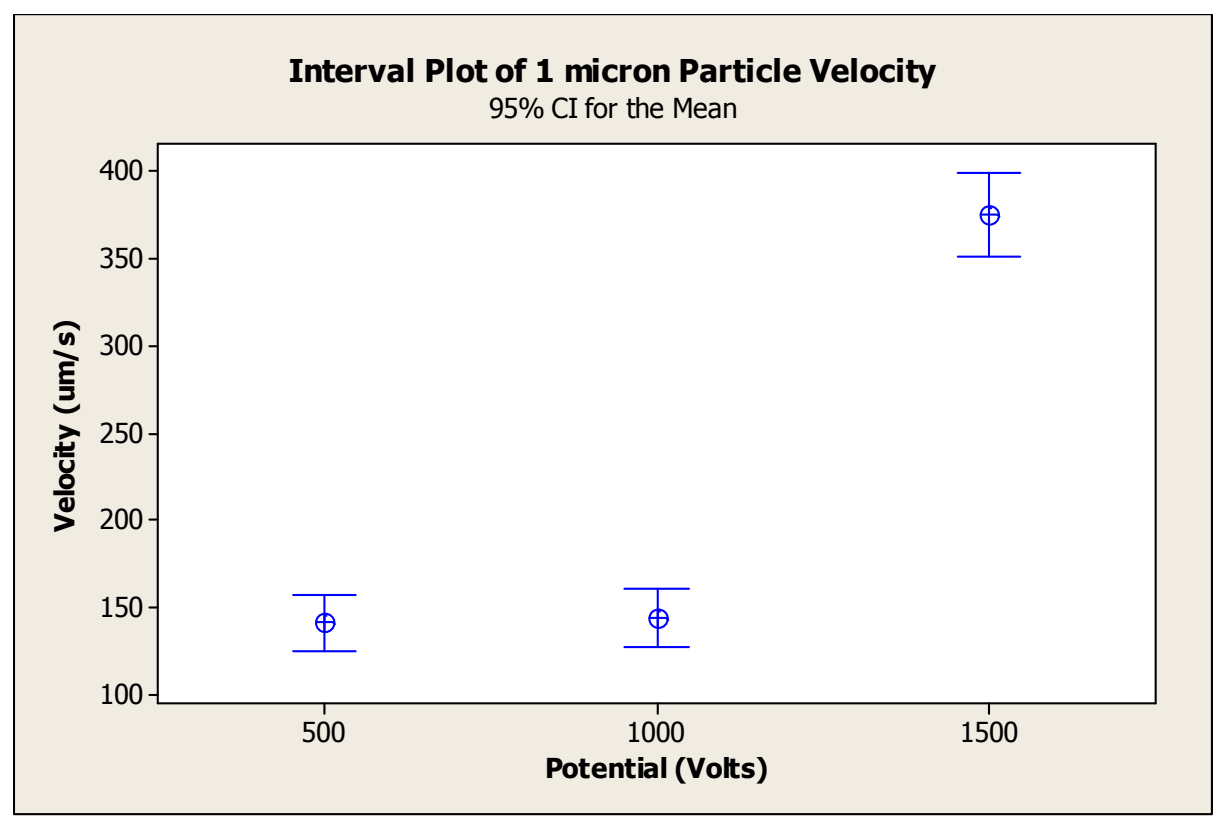

Figure 112 - Interval plot of 1 micron particle velocity with a 95\% confidence level used. The 2000V setting is not shown because data could not be collected for that potential. 


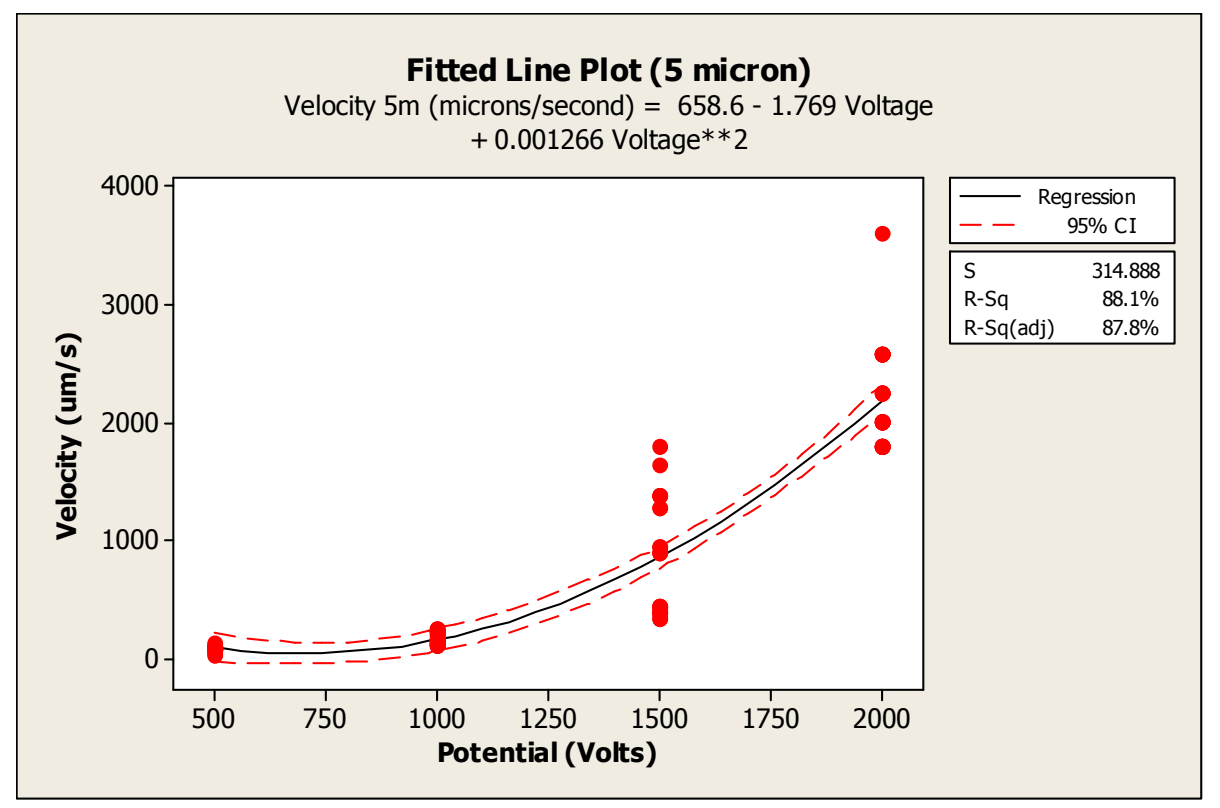

Figure 113 - Scatterplot of velocity versus voltage of 5 micron particles with polynomial line of best fit and $95 \%$ confidence interval. $R^{2}$ values are shown as well.

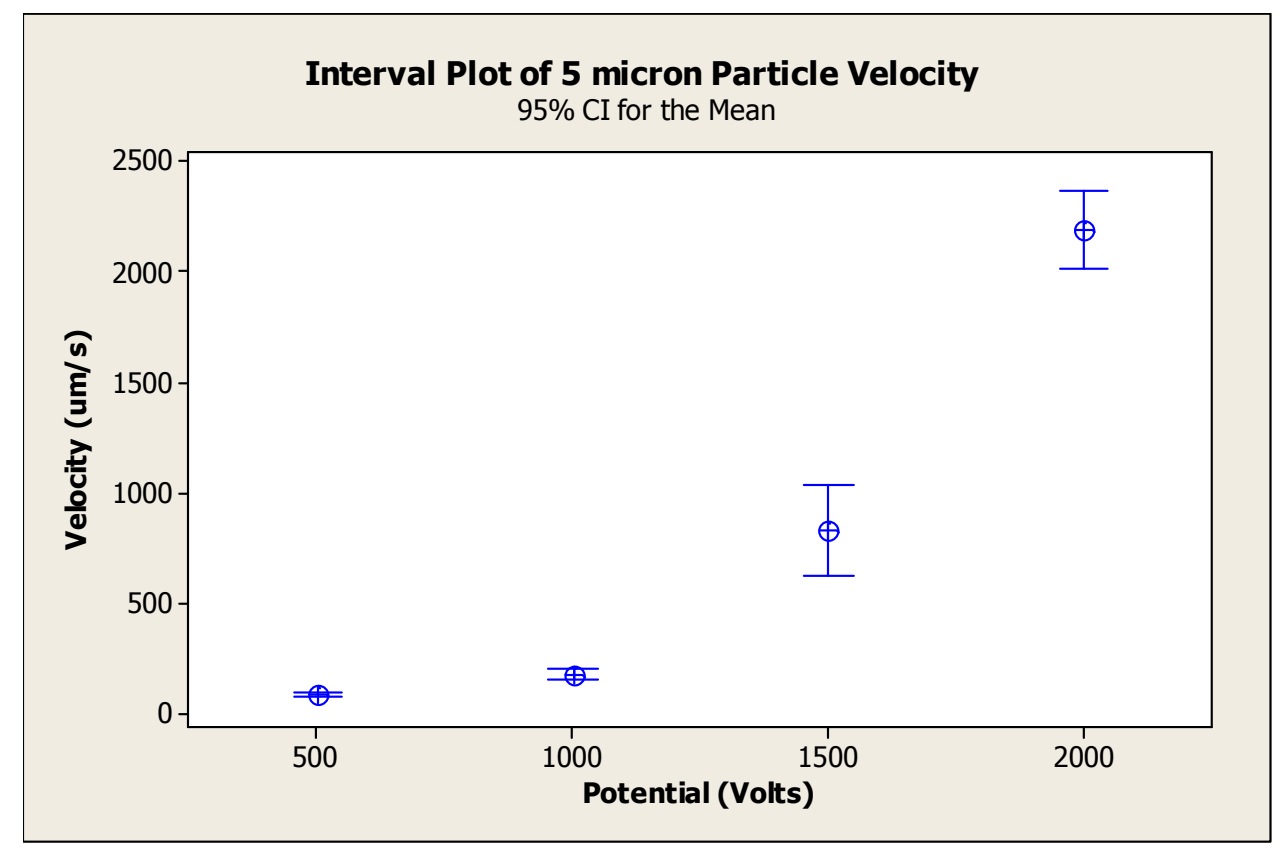

Figure 114 - Interval plot of 5 micron particle velocity with a 95\% confidence level used. 


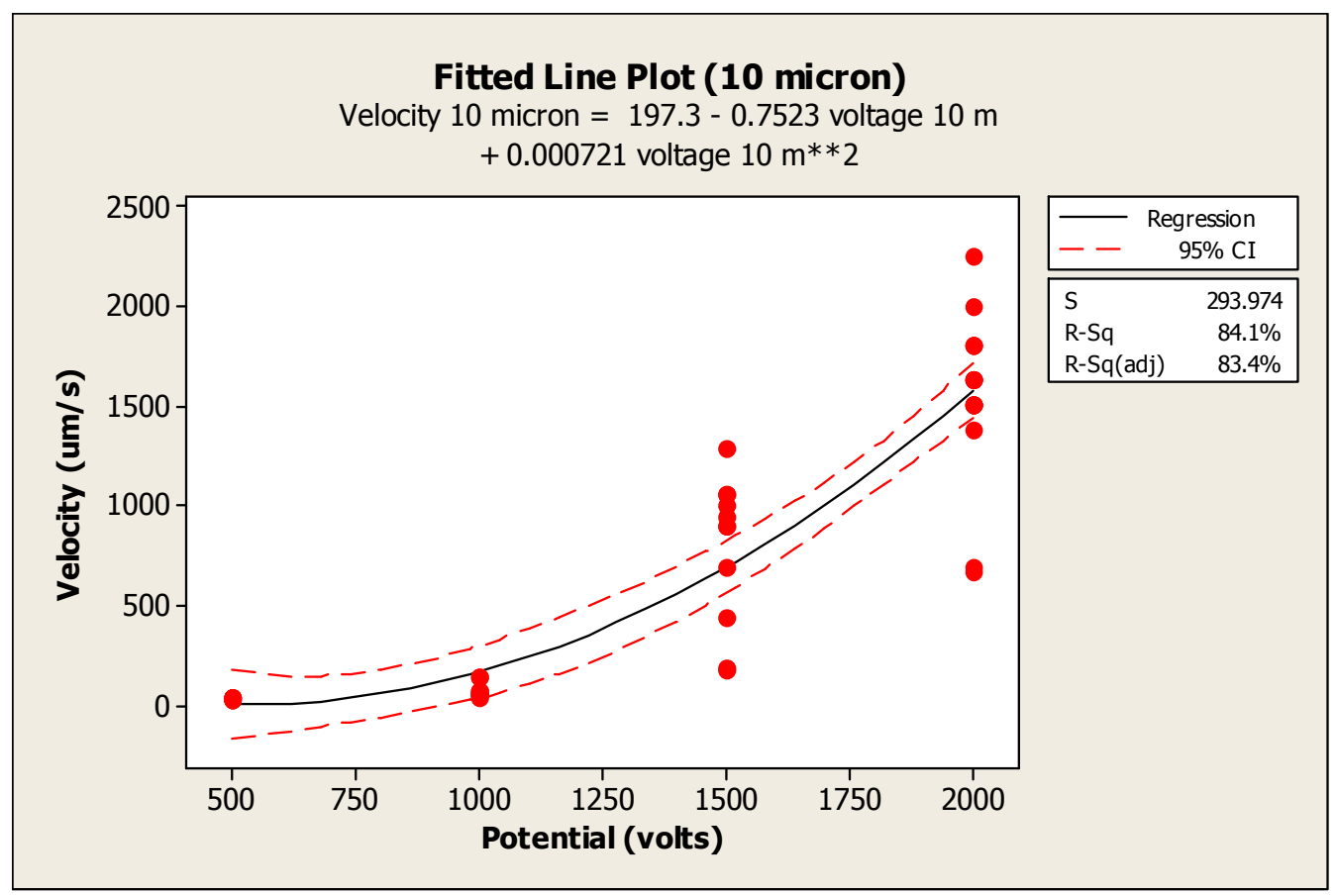

Figure 115 - Scatterplot of velocity versus voltage of 10 micron particles with polynomial line of best fit and $95 \%$ confidence interval. $R^{2}$ values are shown as well

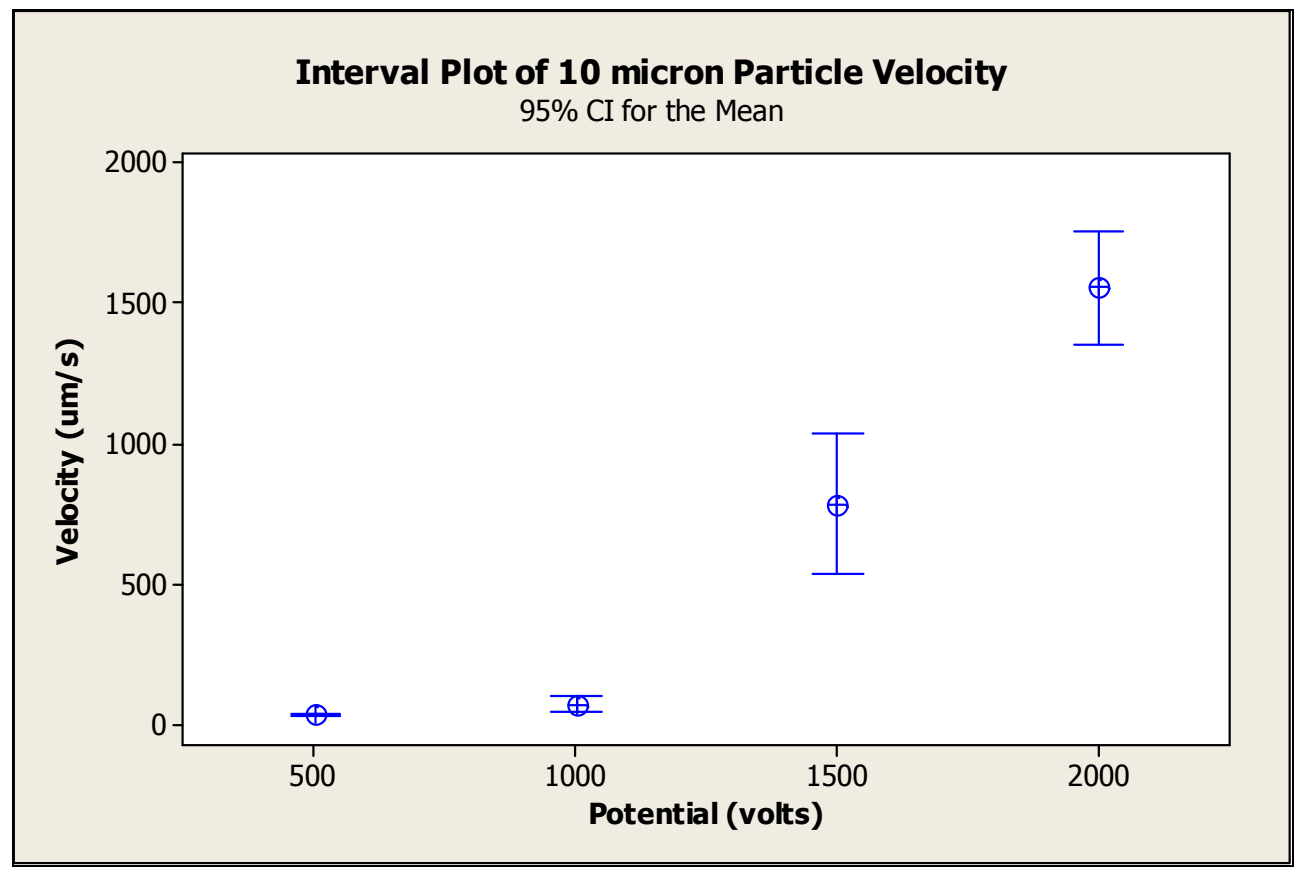

Figure 116 - Interval plot of 10 micron particle velocity with a $95 \%$ confidence level used. 


\section{Discussion}

The results reported in the previous section were a series of experiments designed to test the laser-etched PMMA microfluidic platform created for this thesis. Several different laser etching techniques were performed including multiple low power laser passes, raster cuts, increasing laser powers and post-etching heat treatment. These processes were performed to find the process that created the best-performing CZE chip. The velocity data for three types of microspheres with different electrophoretic was collected at three different points and three or four voltages, depending on sphere size. This data was collected in an attempt to show spheres with different electrophoretic velocities moved at different rate, increasing electric field strengths caused spheres to move at increasing velocities and sphere velocity was independent of data collection location.

\subsubsection{Laser Etching} Microfluidics chips usually contain channels that are much less deep than they are wide facilitate easier focusing and viewing of species of interest within the channels. ${ }^{40}$ Much of the literature on laser etching performs a broad, yet shallow overview of polymer laser

etching applications that cover a range of powers or speeds. ${ }^{33,31,53}$ 


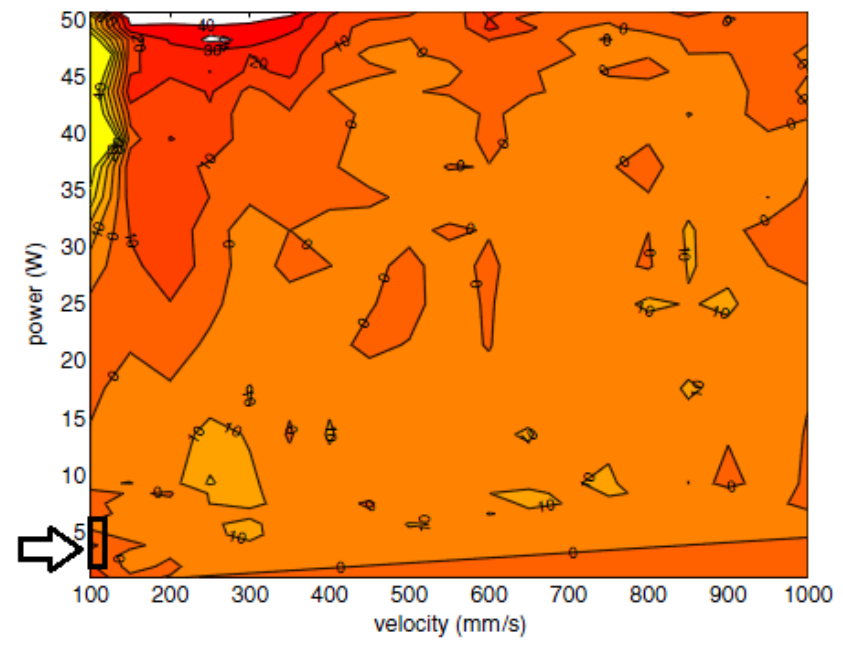

Figure 117 - Graph relating laser translational speed and power with depth indicated by color. Area of interest to this thesis is indicated by the arrow and outline ${ }^{33}$

These papers are beneficial when performing laser etching pilot studies but variances

between laser systems, machine settings and the PMMA manufacturing process do not allow strict usage of equations derived from experimentation of other researchers. Other papers studied can cover the relevant powers of interest, but the speed is much different than the $100 \mathrm{~mm} / \mathrm{s}$ used for all the cuts in this thesis.

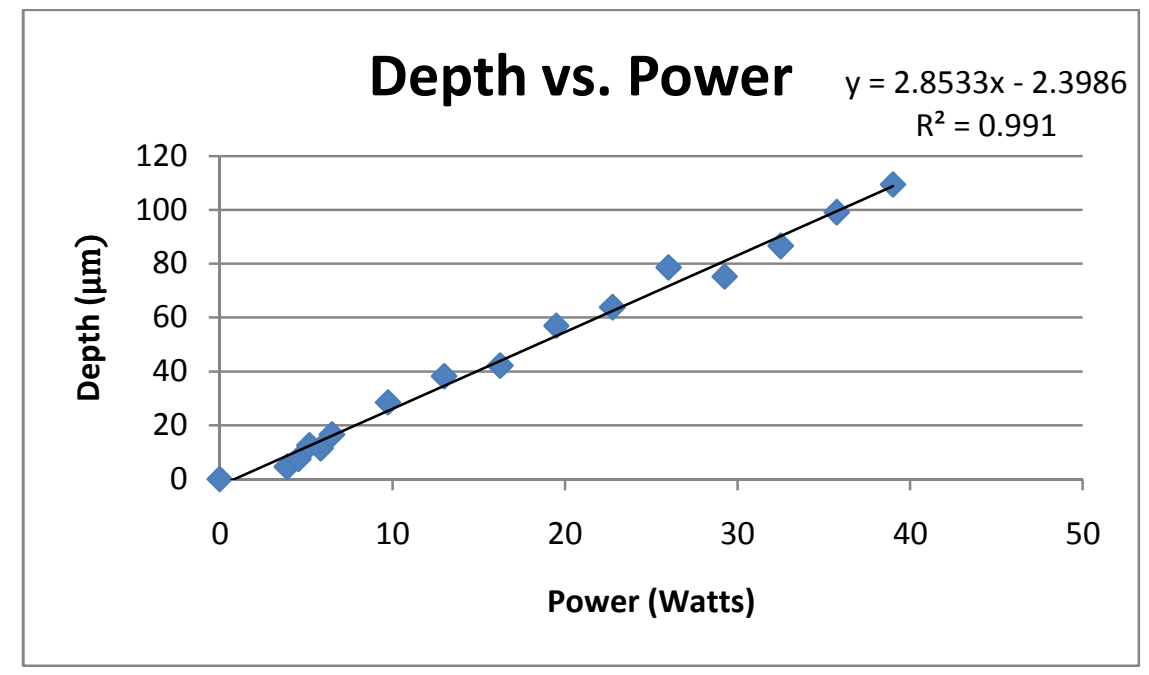

Figure 118 - Depth vs. Power graph of a CO2 laser travelling at $381 \mathrm{~mm} / \mathrm{s}^{31}$ 
Characterizing a laser based on speed and power would be grounds for a project in itself, so this thesis kept the translational speed at a level that created consistent and clean cuts. As the translational speed rose the cuts became more disconnected and irregular, most likely because the airflow rate of the laser cutter's exhaust system could not keep up with the removal of material.

When characterizing the laser it was important to keep in mind the capabilities of the equipment used to evaluate the etched channels. The profilometer from the Materials Engineering department's clean room was used to assess the geometry of the channels after etching. This piece of equipment is able to collect data with a depth range of 400 microns and a width of 1000 microns. The important capability to keep in mind is the maximum step height capability, or its ability to traverse tall architectures while collecting. The profilometer is in contact mode so it will 'drag' along the surface of the material and the extremely fine tip of profilometer runs the risk of breaking at step heights at or above 'jumps' of 100 microns in a short spacing. Additionally, considerations must be made when interpreting the graphed profilometer data. If the architecture of the sample is very fine, the profilometer suffers from what is known as "fat finger" syndrome. The probe at the tip of the cantilever is either a pyramid or triangular prism shape to reduce the chance of breakage. When navigating precise architecture, the probe may incorrectly record data. 

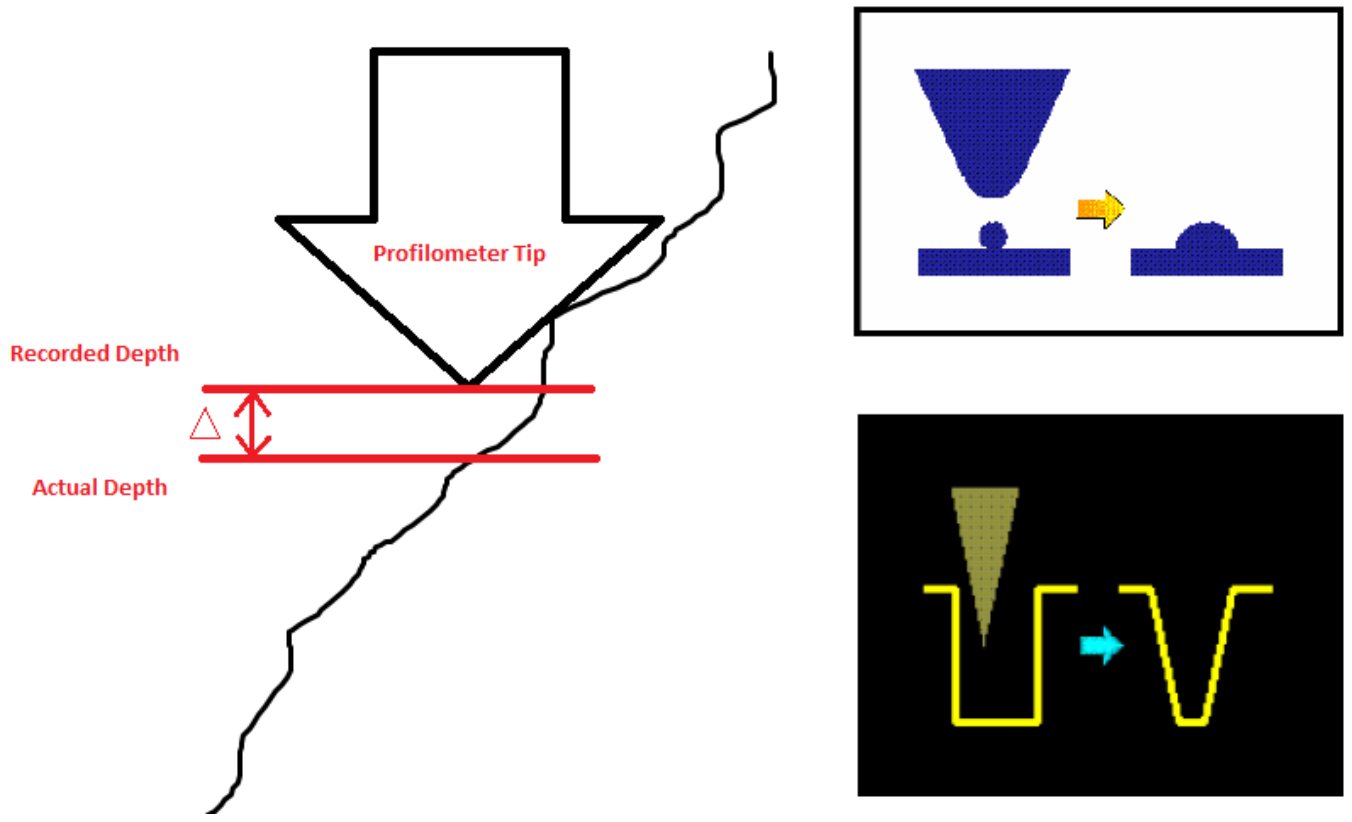

Figure 119 - Three examples of evidence of "fat finger syndrome" inherent to probe-based microscopy 54

Upon close inspection of the graphs of the profilometer data, the channels seem to be skewed in one direction. The left walls of channels appear to have a shallower slope than the right. This is due to the fat finger syndrome and more specifically the one illustrated in the previous figure.

\subsubsection{Increasing Laser Power}

The increasingly steep geometry of the channels with increasing laser power showed that the width of the channel wasn't necessarily increasing. An assessment was made then to use 5 watts of power for the fabrication of all experimental chips. These channels provided enough depth to allow movement of microspheres and any micron-scale foreign objects in the channels. At the same time the channels were not deep enough to waste a great number of microspheres not in the field of focus and instead passing by deeper into 
the channel. Channel width is not as important a factor for CZE. Channel width did not increase greatly with increasing laser power so this was only a minor factor when making the decision. A very shallow channel is subject to clogging due to foreign species entrapment, clumping microspheres or depression of the channel-sealing tape due to elastic pliability. The channels created the 5 Watt power setting struck a good balance between depth and resistance to clogging.

As mentioned in the introduction the laser melts, vaporizes, and then ejects the material out of the channel. Observing the profile for the rastered 2 watt etching shows that a clear channel never really was etched in the material. The redeposited material came out to several microns taller than the base material in some locations. Channels created with a laser power of 1 or 2 watts did not remove material as much as melt it and allow the majority of it to redeposit on the surface. These powers did not reach the 'fluence threshold' to vaporize and eject material. ${ }^{55}$

Once the 3-watt setting is used, real ablation is seen with a fairly significant amount of material removal but there is still an issue with redeposition, especially in raster cutting. It can therefore be said that the 3-watt laser does not transmit enough energy into the material to cause sufficient ejection of material. Thus, with this particular laser cutting machine the 3 watt setting is the critical power that crosses the fluence threshold. Large increases in the amount of material ejected came at the 4 and 6 watt settings where the laser was able to eject large amounts of material. Future researchers are therefore encouraged to use at least 4 watts for channel fabrication. To create chambers with raster 
cuts researchers are encouraged to use at least 5 watts to reduce the chances of channel collapse when sealed with an elastic material and subjected to negative pressures, a problem inherent to PDMS microfluidics chips. ${ }^{56}$

\subsubsection{Multiple Laser Passes}

Some researchers experimented with multiple laser passes ${ }^{31}$. In an attempt to create both deep and wide channels, several passes were made with a low laser power of 3 watts. Instead of making a very deep channel with the width of a single pass, it ended up creating a very steep channel with very sharp bottom geometry due to the 25 micron beam etching directly down the middle of the channel without encountering the resistance

of the extra PMMA. Experimentation down this route was stopped because it created less favorable geometries than higher power laser passes and data collection was halted to stay within the suggested use guidelines of the profilometer.

\subsection{Heat Treatment}

Heat treatment of the material was performed in order to anneal the ablated PMMA and increase the visibility through the channel. Heat treatment was mentioned in literature as a means to smooth the rough channels of the PMMA after laser ablation and hopefully to obtain results similar to Chang et al.. 

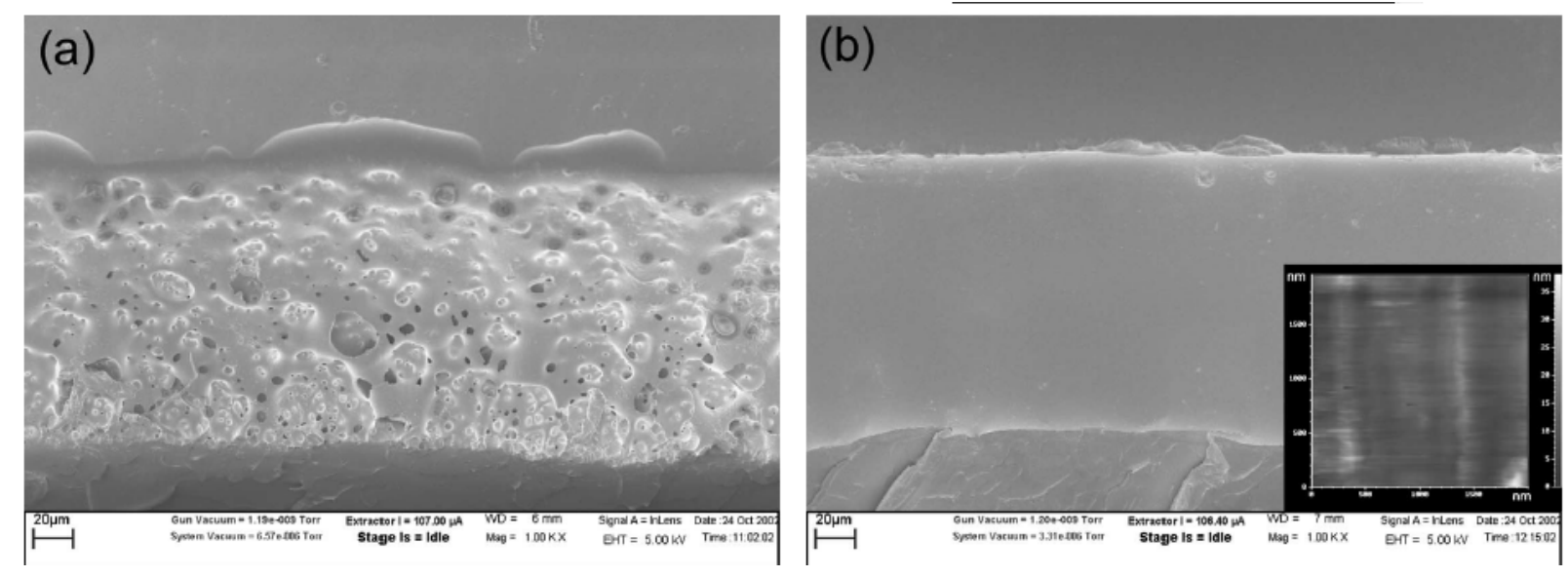

Figure 120 - Laser-etched microchannel before $(\mathrm{L})$ and after $(\mathrm{R})$ thermal annealing at $170{ }^{\circ} \mathrm{C}$ for 30 $\min ^{53}$

The heat treatment of the chips did not change the visual opacity of the chips to a noticeable degree by visual inspection. The experiments were run while the chips were laying face-down on the inverted microscope so visual clarity was not a problem during experimentation. The heat treatment did change the geometry of the channels in that the 'lip' along the edge of the channel lowered after treatment. The edges of the channels became more erratic and the channel deepened. Because this heat treatment step was another level of manufacturing and didn't seem to add any positive functional attributes to this chip design, it was abandoned. Future researchers may want to look into this method for more complicated chips that require more visual clarity.

\subsection{Channel Roughness}

Roughness of channels did not seem to change a great amount between multiple passes and low laser powers until the 5 watt setting was used. When the 5 watt setting was used, the small perturbations in the geometry at the bottom of the channel gave away to the smoother lines between pulses. The laser was set at 1000 pulses per inch and with a 
pulse diameter of 1/1000 of an inch the pulses were sequential. The unevenness in the bottom of the channel is most likely due to the anisotropic nature of the extruded PMMA combined with the redeposited material on the edges of the channels.

\subsubsection{Sphere Electrophoresis}

Siphon effects were something not mentioned in literature and were only found once experimentation had started. This is most likely due to the experimental setup being in its immature stages. The output of the channel ran to a length of tygon tubing and into a waste beaker. Depending on the height of end of the tubing, the flow would either run forward or backward at speeds comparable to the $1500 \mathrm{~V}$ potential setting. The height of the output channel was adjusted until the particle velocities were less than 5 microns per second to as to not skew the results a significant amount. Not having a significant difference between velocities should not be construed as a failure of the platform. Electropherograms are interpreted by the sequence of peaks and time is used as a relative indicator of electrophoretic mobility. In one study, peptide mobility in electrophoresis experiments varied from theoretical values by as much as $22 \%$. 


$$
\begin{array}{r}
0.68 \\
2.45 \\
-22.90 \\
-10.72 \\
-8.10 \\
6.60 \\
-3.49 \\
-13.58 \\
1.94 \\
2.36 \\
10.02 \\
6.14 \\
1.93 \\
2.65 \\
-2.87 \\
-0.57 \\
-6.77 \\
-8.97 \\
-0.18 \\
6.55 \\
-4.48 \\
12.13 \\
4.25 \\
4.27 \\
-2.04
\end{array}
$$

Figure 121 - Percentage deviation of experimental electrophoretic mobilities from theoretical values In CZE experiments, samples fractionate down the length of a channel, allowing charged species with similar electrophoretic mobilities to magnify their separations. The field of view in experimentation was only 600 microns, not allowing for the differences to manifest greatly. For many of the failed tests in the sphere velocity data section, the averages for the data were very different. However, simply comparing averages is not a strong method of comparison. A t-test factors in the sample's mean, average and standard deviation to determine if two sets of data are statistically different. If the averages are different, yet the samples have small sample sizes, overlapping data or large 
standard deviations the test will fail. For all tests a 95\% confidence interval was used as this is the default interval to use.

\subsubsection{Comparison of Electrophoretic Mobility vs. Velocity}

The results at the end of the channel for both the 1 vs. 5 and 5 vs. 10 micron particle comparisons require a bit more explanation. A possible explanation is the weakening electrical potential towards the end of the channel due to loss of potential due to joule heating. Another is the leakage of current from the channel to the metal baseplate on the microscope. This could be caused by fluid leakage between the two tape strips used to seal the channel as described in the methods and materials section. This would show in results at the end of the channel because it would allow time for the fluid to leak The high $1500 \mathrm{~V}$ potential was enough to separate the 1 and 5 particles to the extent that they were statistically different. The 5 and 10 micron particle data definitely shows that there was a change between the beginning/middle and end stages of the chip. The results comparing the 1 and 10 micron particles show that there is definitely a difference in particle movement between these two particle sizes. The electrophoretic mobility of the 1 micron particle is about three times that of the 10 micron particle so it was fairly easy to distinguish these particles by velocity.

\subsubsection{Comparison of Particle Velocity vs. Data Collection Point}

The 1 micron spheres had a very wide spread of velocities with standard deviations of $33.04,41.56$ and 53.82 for the $500 \mathrm{~V}, 1000 \mathrm{~V}$ and $1500 \mathrm{~V}$ potentials respectively. Because of this high variability, the T-test delivered lower values which disagreed with the hypothesis that the particles moved at the same speed regardless of position. Overall, a 
bit less than half the t-tests delivered a results that disagreed with the hypothesis. For pioneering a fabrication method new to Cal Poly, this can be deemed a positive result.

\subsubsection{Comparison of Particle Velocity vs. Voltage}

For the 1 micron spheres, nearly all the data agreed with the hypothesis that different voltages cause the spheres to move at different speeds. The only result that disagrees with the hypothesis is the $500 \mathrm{~V}$ vs. $1000 \mathrm{~V}$ data at the end of the channel. The other locations that the data was taken from show a slight, but statistically insignificant result from the t-test. The results at the end of the channel also have elevated but still statistically insignificant t-test results. The combination of these two factors - the end of the channel and a lower voltage, were probably the causes of the failed t-test. However, 8 out of 9 tests passed can still be considered a positive result.

The 5 micron sphere data passed all of the t-tests with a 95\% confidence level. The only result that came close to failing the test was again the $500 \mathrm{~V}$ vs. $1000 \mathrm{~V}$ comparison at the end of the channel.

The 10 micron spheres only failed 2 tests at a $95 \%$ confidence level. One of the failures was very close to passing, as the t-test with the hypothesis that the spheres moved faster at $1000 \mathrm{~V}$ than $500 \mathrm{~V}$ gave a $\mathrm{P}$ value of 0.058 , very close to the .05 needed to pass. The $1500 \mathrm{~V}$ vs. $2000 \mathrm{~V}$ failure was not very close to passing and could have resulted from wider data intervals at the two voltage settings.

\subsubsection{Comparison of Velocities vs. Calculated}

Perhaps the most important part of proving the real-world usability of this platform is comparing the experimental values against the calculated values. First, the values for 
individual spheres were compared against the expected. The electric field strength was found by dividing the voltage by the channel length as V/8.5 per electrophoretic standards. The electrophoretic mobilities calculated in the introduction section were multiplied by electric field strengths. For simplicity's sake, the voltage settings for the data are listed instead of electric field strengths.

Table 12 - Expected velocities in microns/second of microspheres

\begin{tabular}{|l|r|r|r|r|r|}
\hline Particle Size & Electrophoretic Mobility & $\mathbf{5 0 0 V}$ & $\mathbf{1 0 0 0 V}$ & $\mathbf{1 5 0 0 V}$ & $\mathbf{2 0 0 0 V}$ \\
\hline $\mathbf{1}$ micron & 38.63 & 2272.24 & 4544.47 & 6816.7 & 9088.96 \\
\hline $\mathbf{5}$ micron & 24.4 & 1435.29 & 2870.59 & 4305.88 & 5741.18 \\
\hline $\mathbf{1 0}$ micron & 12.1 & 711.76 & 1423.53 & 2135.29 & 2847.06 \\
\hline
\end{tabular}

Data could not be collected for the 1 micron spheres under the influence of the $2000 \mathrm{~V}$ source so there were no calculations for that particular data block. The 1 micron particles had roughly 3 times the mobility of the 10 micron particles, which only took 6 frames of animation to cross the field of view. The combination of factors of the speed of the particles, the interlacing of lines by the software to create a full image and the small size of the particles led to the inability to experimentally determine the velocities of the 1 micron particles. The errors were calculated using the standard percent error equation shown below.

$$
\% \text { Error }=100 \cdot \frac{(\text { Experimental }- \text { Theoretical })}{\text { Theoretical }}
$$

First, the averages of the different voltages and sphere sizes regardless of location were compared to the calculated values. 
Table 13 - Percent error difference of experimental electrophoretic velocities from theoretical

\begin{tabular}{|l|r|}
\hline Voltage & \% Error from Theoretical \\
\hline 1m 500v & -93.80407774 \\
\hline $\mathbf{1 m ~ 1 0 0 0 v}$ & -96.84282429 \\
\hline $\mathbf{1 m ~ 1 5 0 0 v}$ & -94.49449322 \\
\hline $\mathbf{5 m ~ 5 0 0 v}$ & -94.20202483 \\
\hline $\mathbf{5 m ~ 1 0 0 0 v}$ & -93.85459096 \\
\hline $\mathbf{5 m ~ 1 5 0 0 v}$ & -80.70341603 \\
\hline $\mathbf{5 m ~ 2 0 0 0 v}$ & -61.79762223 \\
\hline $\mathbf{1 0 m}$ 500v & -95.38995763 \\
\hline $\mathbf{1 0 m}$ & -95.00319084 \\
$\mathbf{1 0 0 0 v}$ & \\
\hline $\mathbf{1 0 m}$ & -63.24749458 \\
$\mathbf{1 5 0 0 v}$ & \\
\hline $\mathbf{1 0 m}$ & -46.91769269 \\
$\mathbf{2 0 0 0 v}$ & \\
\hline
\end{tabular}

The percentage difference was very large but interestingly all are negative, indicating all the values were less than the theoretical. This indicates the platform was not effective at moving spheres as fast as theoretically possible. Closer observation of the errors in each section revealed trends and possibly the use of a correction factor to use in future experimentation. 
Table 14 - Comparison of theoretical and experimental values for sphere velocity data. Velocities are in microns/second

\begin{tabular}{|l|r|r|r|}
\hline $\begin{array}{c}\text { Velocities are in } \\
\boldsymbol{\mu m} / \mathbf{s}\end{array}$ & Theoretical & Experimental & $\begin{array}{r}\text { Ratio T/E } \\
\text { (Correction } \\
\text { Factor) }\end{array}$ \\
\hline $\mathbf{1 m} \mathbf{5 0 0 V}$ & 2272.235294 & 140.7871858 & 16.13950361 \\
\hline $\mathbf{1 m} \mathbf{1 0 0 0 V}$ & 4544.470588 & 143.4781987 & 31.67359661 \\
\hline $\mathbf{1 m} \mathbf{1 5 0 0 V}$ & 6816.705882 & 375.2975454 & 18.16347047 \\
\hline $\mathbf{5 m} \mathbf{5 0 0 V}$ & 1435.294118 & 83.2025 & 17.25061287 \\
\hline $\mathbf{5 m ~ 1 0 0 0 V}$ & 2870.588235 & 176.3765385 & 16.27534059 \\
\hline $\mathbf{5 m ~ 1 5 0 0 V}$ & 4305.882353 & 830.7334783 & 5.183229598 \\
\hline $\mathbf{5 m} \mathbf{2 0 0 0 V}$ & 5741.176471 & 2192.8575 & 2.618125651 \\
\hline $\mathbf{1 0 m} \mathbf{5 0 0 V}$ & 711.7647059 & 32.82417809 & 21.68415928 \\
\hline $\mathbf{1 0 m} \mathbf{1 0 0 0 V}$ & 1423.529412 & 71.15602886 & 20.00574561 \\
\hline $\mathbf{1 0 m} \mathbf{1 5 0 0 V}$ & 2135.294118 & 785.0496944 & 2.719947709 \\
\hline $\mathbf{1 0 m} \mathbf{2 0 0 0 V}$ & 2847.058824 & 1511.815268 & 1.883205497 \\
\hline
\end{tabular}

The ratio of theoretical to experimental can also be called a 'correction factor' - a

number that can be multiplied to the electrophoretic velocity in future experiments to obtain more reasonable data. Observing this ratio brings up a few trends. First, the 1 micron sphere correction factor hovers within a fairly small range. Next, the low voltage experiments with the larger spheres reveal even smaller ranges for the two particle sizes.

Last, the high voltage correction factors for the larger spheres also fit within a small range. The ratios were then compiled into the following table.

Table 15 - Correction factors for sphere velocities based on size and voltage

\begin{tabular}{|l|r|}
\hline & $\begin{array}{l}\text { Correction } \\
\text { Factor }\end{array}$ \\
\hline $\begin{array}{l}\text { Average Small Sphere }(\leq \mathbf{1} \\
\boldsymbol{\mu m})\end{array}$ & 20.61984 \\
\hline $\begin{array}{l}\text { Average Large } \\
\text { Sphere }(>\mathbf{1} \boldsymbol{\mu m}) \\
\text { Low Voltage }(\leq \mathbf{1 0 0 0 V})\end{array}$ & 18.30165 \\
\hline $\begin{array}{l}\text { Average Large Sphere } \\
(>\mathbf{1} \boldsymbol{\mu m})\end{array}$ & \\
Low Voltage $(>\mathbf{1 0 0 0 V})$ & \\
\hline
\end{tabular}


The velocities were multiplied by the correction factors and individually compared against the theoretically determined velocity. In addition to the simple numerical correction factor, a voltage-dependent single-order equation was spawned from the data to try and find a model that would better fit the predicted values and create an equation that could be applied to future experiments. The data from the 5 and 10 micron correction factors for each voltage were graphed and a line of best fit was drawn through the data.

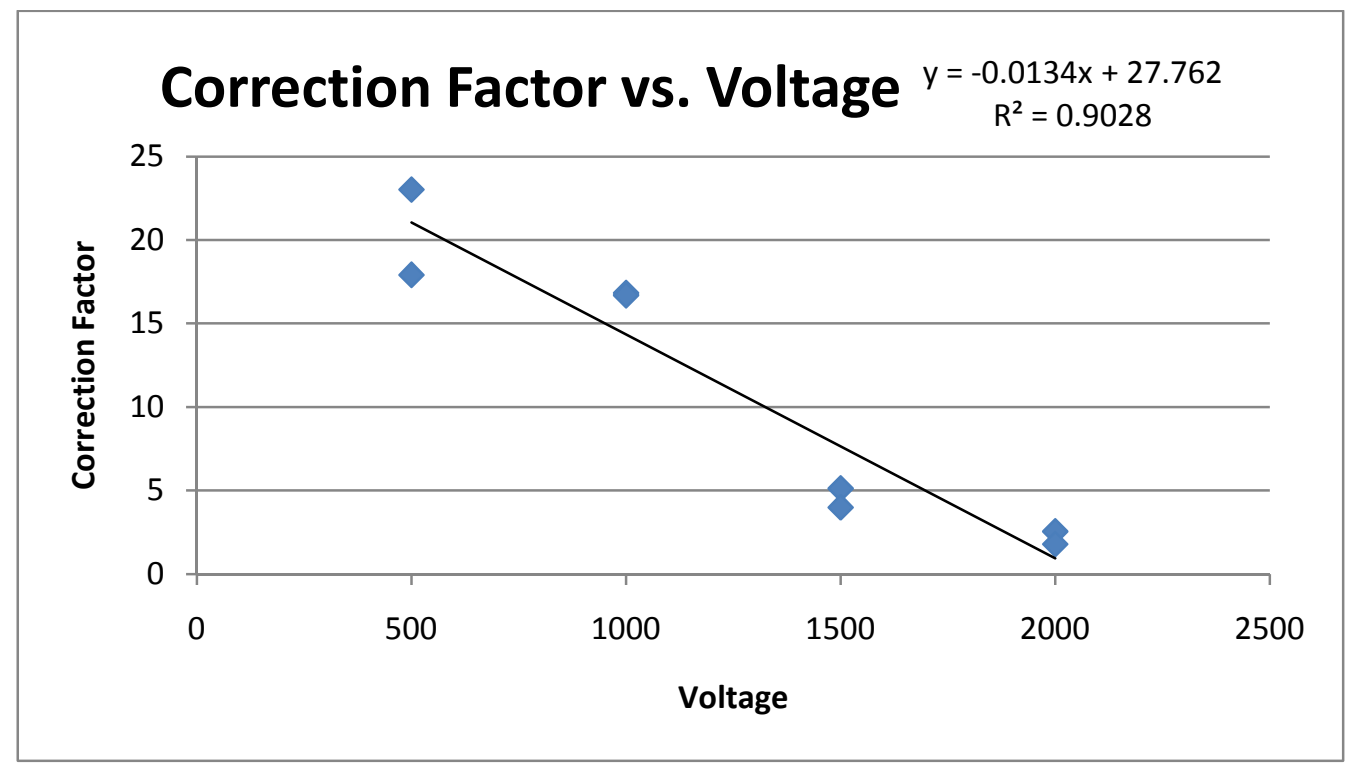

Figure 122 - Graph of correction factor versus voltage for 5 and 10 microsphere data

With a strong $\mathrm{R}^{2}$ value of .9028 , the voltage dependent correction factor showed promise. The data points were again multiplied by the correction factor and the error between the corrected data points and the theoretical velocity values was calculated. For both correction factor types, the regular experimental errors were averaged and the absolute values of the experimental errors for the larger spheres were averaged as they used a pair 
of pooled correction factor. The absolute values for the percent error are a stricter way to determine effectiveness of a correction factor. Some error percentages will be above or below the accepted value and overall they will yield a result that is closer to zero than the average absolute error percentage. The 1 micron correction factor was taken from only the 1 micron data, so the non-absolute data will be very close to zero.

Table 16 - Average percent error from theoretical values for corrected data compared to original error

\begin{tabular}{|l|r|r|r|}
\hline Size and Voltage & Average \% Error (Corrected) & $\begin{array}{l}\text { \% Error } \\
\text { (Original) }\end{array}$ & Improvement \\
\hline $\mathbf{1 m}$ & -0.0000359 & -95.04713175 & 95.04709585 \\
\hline $\mathbf{1 m}$ Absolute & 25.28325778 & -95.04713175 & 69.76387397 \\
\hline $\mathbf{5 m}$ & -1.21399755 & -82.63941351 & 81.42541596 \\
\hline $\mathbf{5 m}$ Absolute & 28.34570558 & -82.63941351 & 54.29370793 \\
\hline $\mathbf{1 0 m}$ & 16.61882986 & -75.13958394 & 58.52075408 \\
\hline $\mathbf{1 0 m}$ Absolute & 42.71149867 & -75.13958394 & 32.42808527 \\
\hline Equation-dependent & & & \\
\hline $\mathbf{5 m}$ & & & \\
\hline $\mathbf{5 m}$ abs & -1.996053009 & -82.63941351 & 80.6433605 \\
\hline $\mathbf{1 0 m}$ & 48.16520625 & -75.13958394 & 26.97437769 \\
\hline $\mathbf{1 0 m}$ abs & 15.30310666 & -82.63941351 & 67.33630685 \\
\hline
\end{tabular}

The average error of the flat correction factor beat out the equation-dependent correction factor in every category. It is safe to assume the numerical correction factor is superior from this data. Therefore, the equation for electrophoretic mobility for future experimentation with laser-etched PMMA should be

$$
\frac{2}{3} \frac{\zeta \varepsilon E_{x}}{\mu} F
$$


Where $\mathrm{F}=20.62$ for small spheres $(\leq 1 \mu \mathrm{m}), 18.3$ for large spheres $(>1 \mu \mathrm{m})$ at low electric fields $(\leq 120 \mathrm{~V} / \mathrm{cm})$ and 2.866 for large spheres at high electric fields $(>120 \mathrm{~V} / \mathrm{cm})$.

After analysis of the data, the error percentages fit within the $70 \%$ error margin soft goal of this thesis. Analysis of the data as a whole shows that this platform is an immature, yet effective way of producing microfluidic chips. Future experimentation and iterations of the platform will inevitably produce chips that produce results with results closer to the theoretical electrophoretic values and with tighter data grouping. PDMS microfluidics may have the current advantage of known manufacturing potential but PMMA and other mechanically strong device-worthy polymers will inevitably become the material of choice for field microfluidic devices.

\subsubsection{Alternative Manufacturing Methods}

Only a fraction of the types of possible microfluidic manufacturing methods were attempted in this thesis. In this section are many related methods that show promise as directions of future projects.

\subsubsection{Hybrid Systems}

Hybridized manufacturing methods have evolved out of the search for alternative methods for microfluidic platform manufacturing. ${ }^{50}$ PMMA or metal can be laser-etched into the inverse of the microfluidic architecture and used as a mold for PDMS. ${ }^{51}$ This method is difficult to create precise architecture with, but would be an excellent in-house prototyping method to use before creating a costly mold that requires waiting. ${ }^{31}$

Interfaces between PMMA slides may be difficult to seal due to inherent width tolerances from the manufacturing process. The thicknesses of extruded PMMA can vary from 
$+10 /-15$ microns throughout its length. ${ }^{52}$ One solution to this problem is adding a very thin layer of PDMS at the interface of the two sheets of PMMA that acts as a sealant.
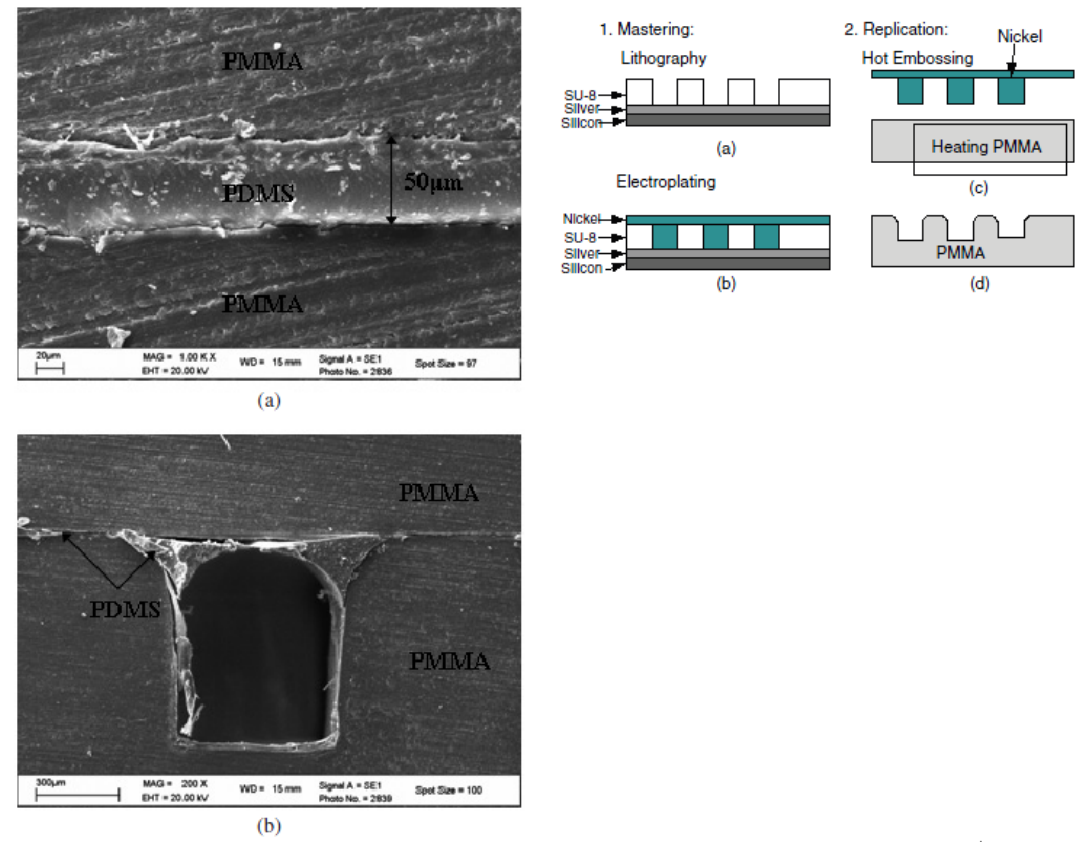

Figure 123 - Hot embossed PMMA microfluidic device with 50 micron PDMS layer at the interface ${ }^{50}$

Adding PDMS at the interface of the two layers works to seal voids that would otherwise cause leakages. ${ }^{50}$

\subsubsection{Laser Etched Tape}

The use of laser-etched tape is gaining ground in the world of microfluidics. Two major manufacturing methods have arisen from this method: tape-based molds and gasket-based microfluidics. The idea is to use tape and a laser cutter to create architecture similar to that of soft lithography methods. Parallel steps between this process and soft lithography are explained in the following figure 

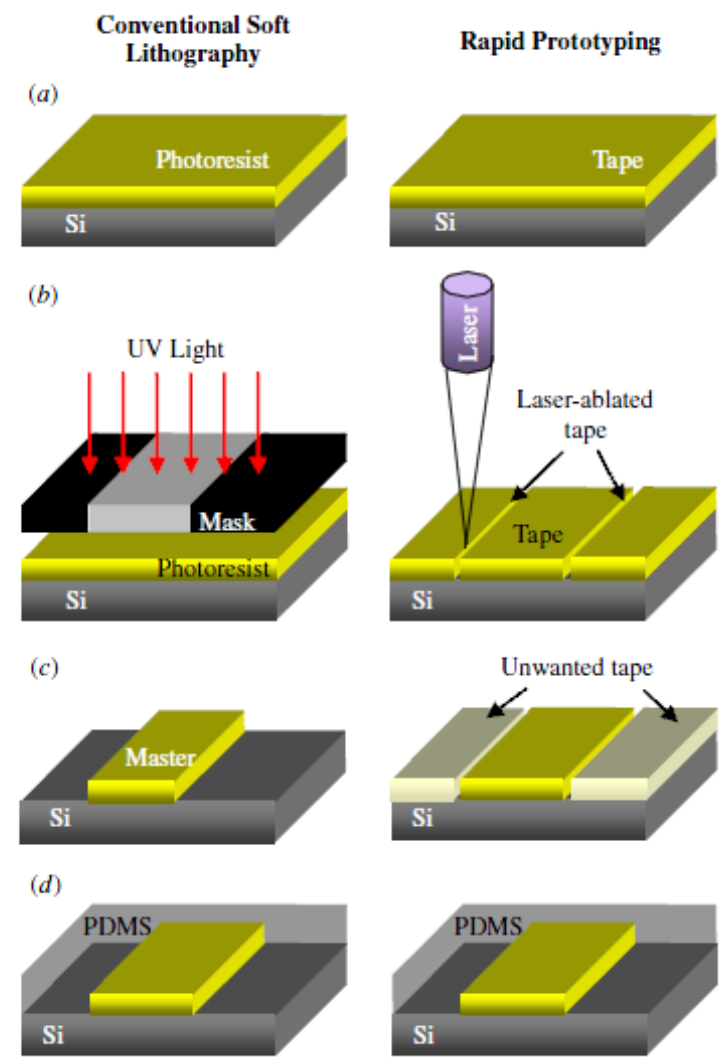

Figure 124 - Tape-based microfluidics device manufacturing process ${ }^{58}$

The etched tape was removed and the tape-on-silicon was used as a PDMS mold. ${ }^{58}$ This method of manufacturing creates chips with much smoother surfaces than direct etching of PMMA. 

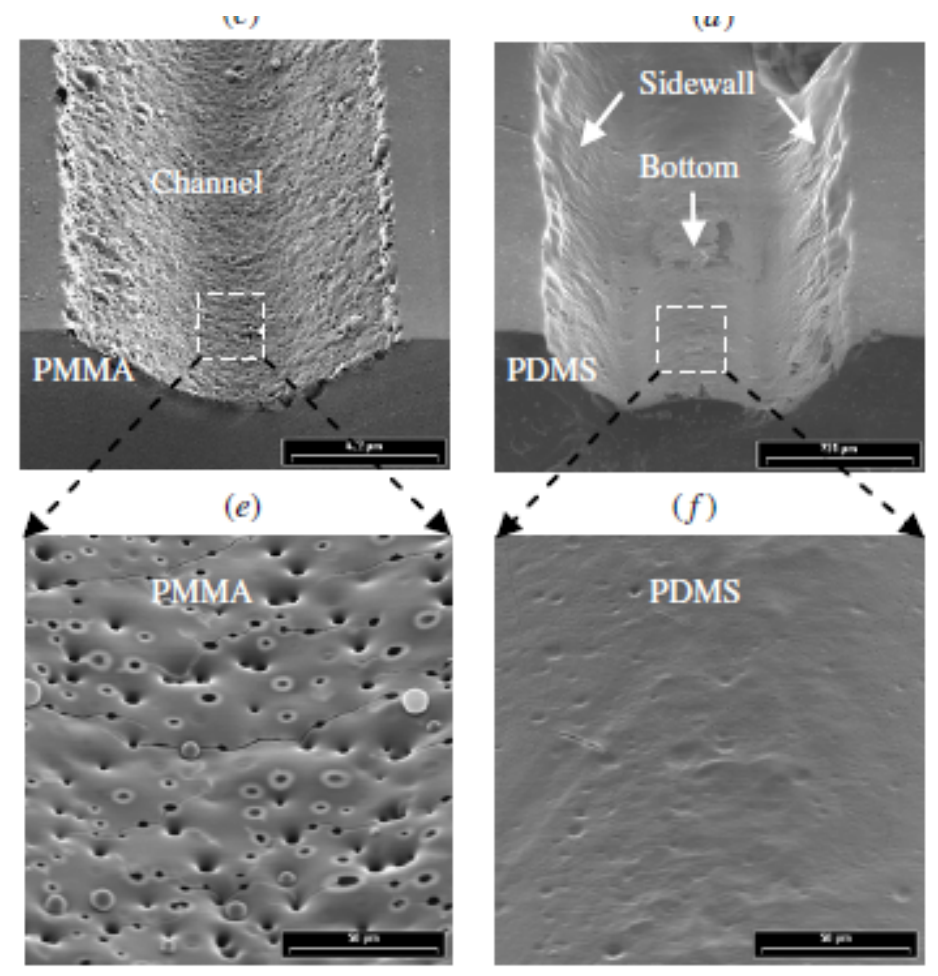

Figure 125 - SEM comparison of surface roughness between direct-etched PMMA (L) and PDMS poured into a laser-etched tape mold $(R){ }^{58}$

Similar manufacturing methods have been used to create microfluidic chips that use the tape as a gasket sandwiched between two slides of glass. ${ }^{59}$

\subsubsection{Hot Embossing}

Hot embossing has shown to be very promising in the area of low-cost microfluidics with metal molds. ${ }^{60,61,62}$ and hot wire embossing. ${ }^{63}$ In keeping with the low-cost theme, a method for manufacturing devices using small-gauge wires imprinting channels into PMMA sheets and glass has been performed. Wire embossing, like laser-etching, 'moves' material out of the channel to the edges to create a lip that helps seal the channels. Results for the wire embossed PMMA chips performed at or superior to professional methods of manufacture. ${ }^{63}$ 

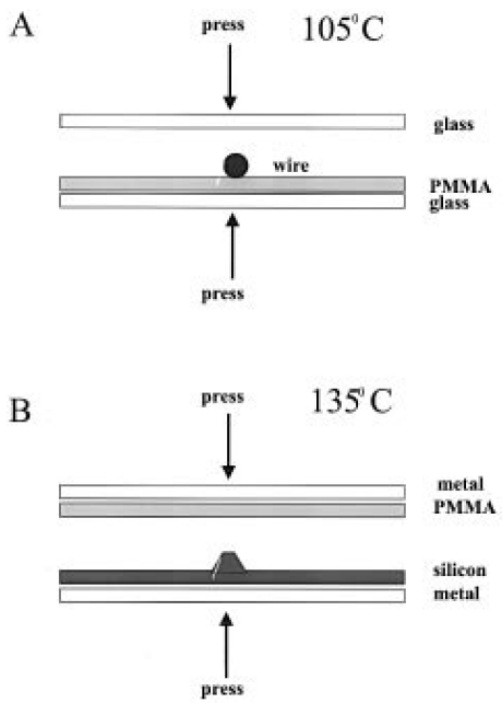

Figure 126 - Fabrication method for wire embossing compared to embossing method with silicon mold $^{64}$

Metal molds can be created a variety of ways - reactive ion etching, CNC machining, electroplating, vapor deposition and sputtering to name just a few although there are more covered in an extensive literature review on this topic. ${ }^{65} \mathrm{CNC}$ machining has been used to create both polymer injection molds and imprinting molds on the scale of tens of microns. ${ }^{66,67}$ The advantage of PMMA is its mechanical strength at the microscale. High aspect ratio structures have been manufactured using hot embossing with PMMA. 


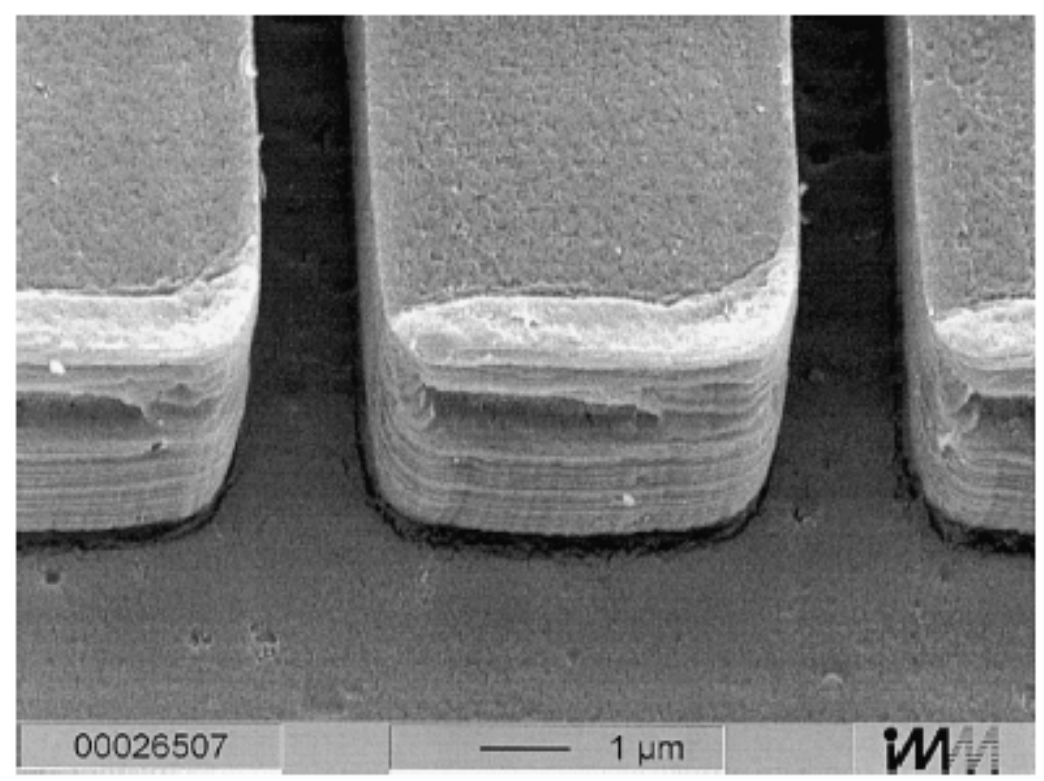

Figure 127 - Microarchitecture created in PMMA by hot embossing ${ }^{68}$

Creating molds of silicon for polymers using established lithography methods has also been proven as a reliable manufacturing method. ${ }^{69}$

\subsubsection{Patterning Electrodes}

Creating a functional electrode system was one of the more complicated parts of this project. Although the final method of using in-line hypodermic tubing ended up performing admirably, there will inevitably be microfluidic ideas that require on-chip electrodes at a finer scale or of more precise geometry. Patterning electrodes onto glass is a long standing method for creating reliable electrical contacts between specific areas of a chip and the experimental setup. Screen printing of electrodes has shown to be a reliable and very cost-effective method for patterning electrodes onto PMMA. ${ }^{70}$ Yet another method for embedding electrodes into chips is the use of conductive solder wire in concert with mold-based manufacturing methods. ${ }^{71}$ 


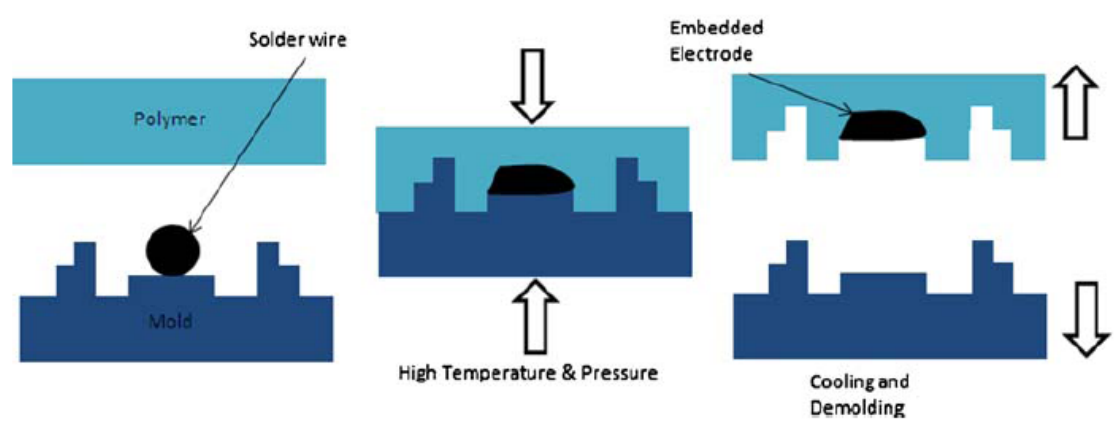

Figure 128 - Embedding solder wire into microfluidic chip as an electrode ${ }^{71}$

An idea only mentioned in a few articles was the use of conductive films cut either

manually or by a laser cutter that can be sandwiched between the chip and its backing. ${ }^{72}$ 3M sells such films under the model number $8880 \mathrm{~S} 3$ and $8880 \mathrm{~S} 7$ that are 3 and 7 thousands of an inch thick respectively.

\subsection{Other Microfluidics Chips Manufacturable With Laser Etched PMMA}

When proving a new microfluidic platform, CZE is usually the test subject to prove the viability of that platform. Refinement of the CZE experimentation is still possible but branching out from the initial project with slightly more complicated projects should be encouraged. Below are several different ideas accompanied by AutoCAD drawings for future students wishing to build on the research performed in this project.

H-Filters are a longstanding standard within microfluidics as a way to separate analytes like cells and proteins from a fluid sample without the need for a membrane filter subject to biofouling. This is a simple design that can be easily replicated with a laser-etched PMMA chip. The channels leading into the separation section can be simple vector cut channels and the separation channel can be widened to give ample room for separations. 


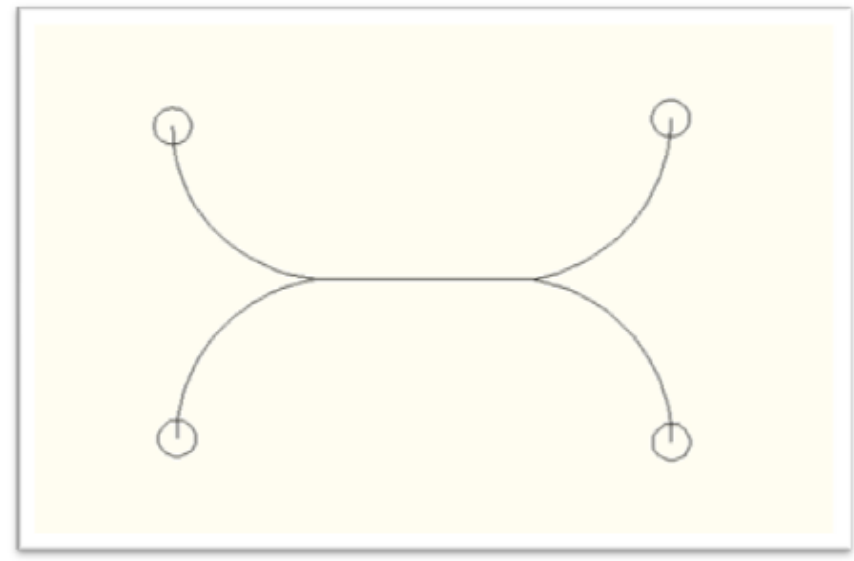

Figure 129 - AutoCAD drawing of a proposed H-Filter for fabrication with a laser cutter

More complex channel experiments can be repurposed from their PDMS counterparts, such as the enzyme analysis chip shown below. This chip tests enzyme activity by altering the influx of enzyme inhibitor and analyzing the concentrations of substrate and products down the channel.

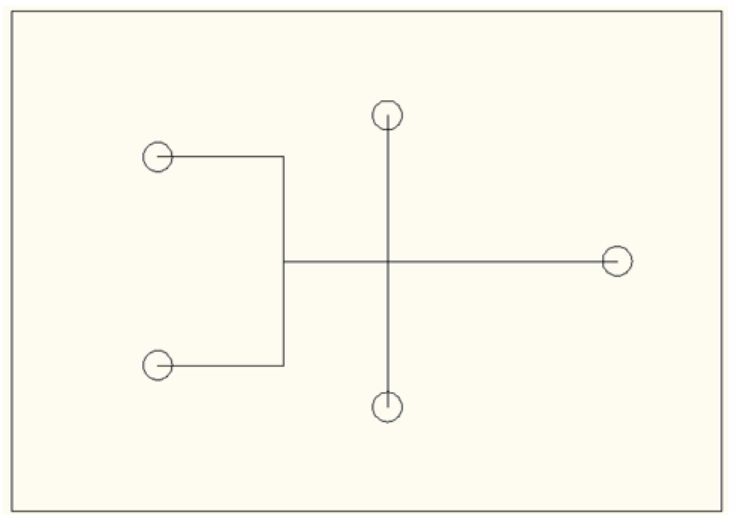

Figure 130 - Enzyme analysis chip. Starting from the topmost circle, the functions of the inlets/outlets are enzyme inhibitor, waste, enzyme, substrate, and buffer. Based on the work by Hadd et. Al. ${ }^{73}$ 
This chip can be made more complicated with the addition of more complex geometries, such as the one suggested provided by Dr. J. K. Park and reconstructed using AutoCAD. 74

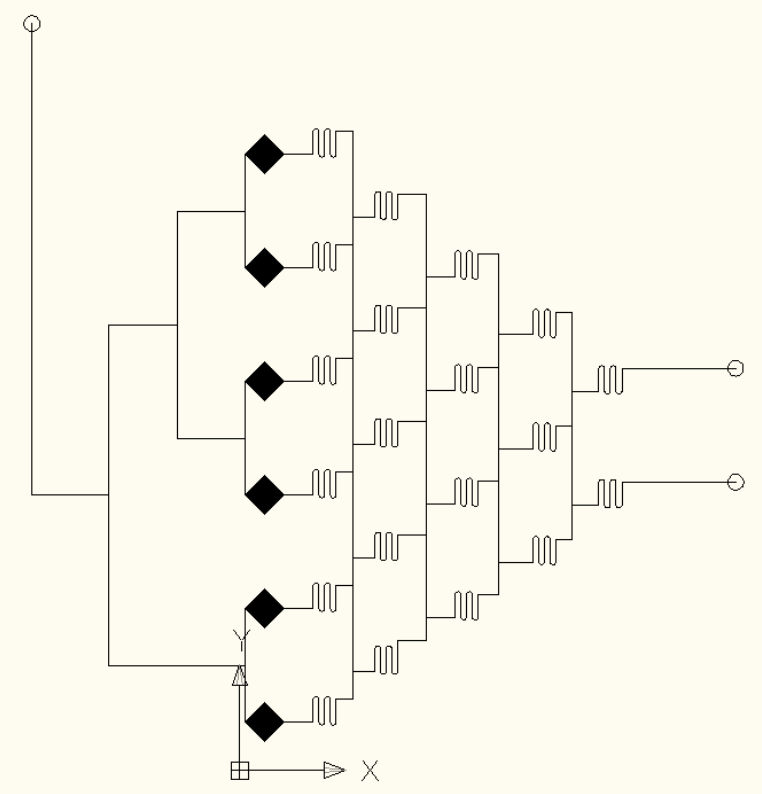

Figure 131 - More complicated enzyme assay. The left injection port is for the enzymes, the black diamonds are reaction chambers, the loops in the channel network are for the dilution of the substrate and the two ports on the right are for substrate injection (top is low concentration, bottom is high) ${ }^{74}$

Microfluidics experiments from within the same thesis group can be created using laseretched PMMA. Below is the experimental chip design by David Pescetti for the creation of a Micellular Electrokinetic Chromatography chip. With laser etching fabrication the different chamber depths, shown in different colors, can be fabricated in the same run of the laser cutter. When manufacturing with in PDMS, the process requires an aligner and three different masks. Additionally, PDMS requires many posts within the chambers to 
keep the PDMS from bowing and collapsing the channel. PMMA is a much stiffer material and can be fabricated without this design constraint. ${ }^{75}$

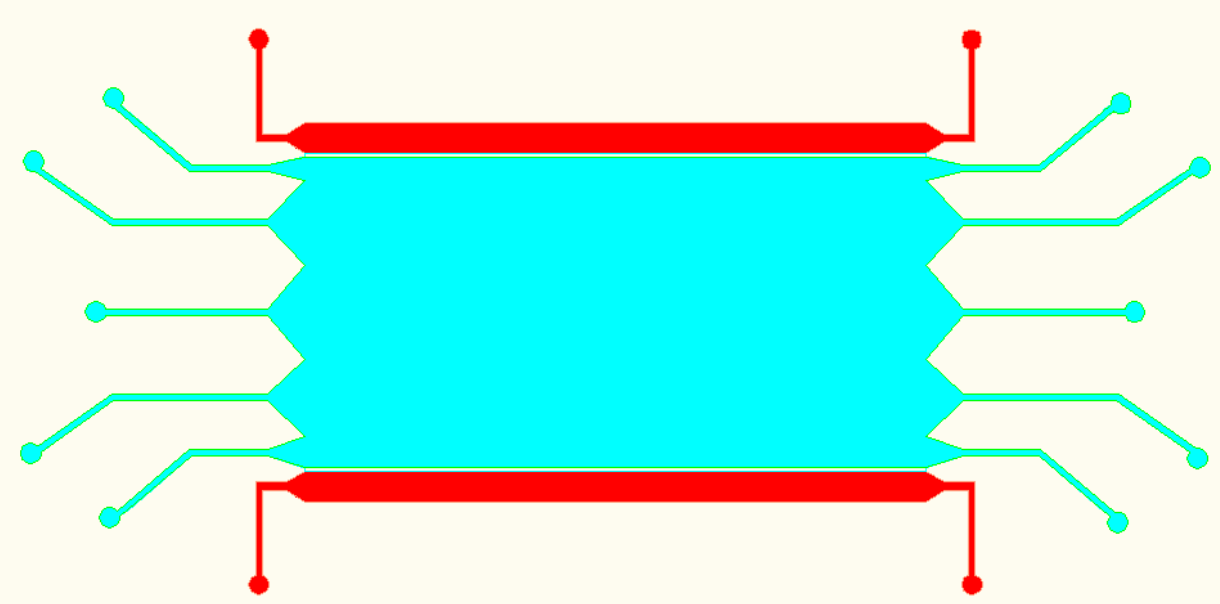

Figure 132 - Design of microfluidic chip adapted from David Pescetti's work in MEKC. ${ }^{74}$

As mentioned in the introduction, mixing of fluids under laminar flow is a fundamental problem with microfluidics. Based on the paper by Liu et al. about passive micromixing using a 3-dimensional serpentine geometry, the following chip was designed. ${ }^{77}$

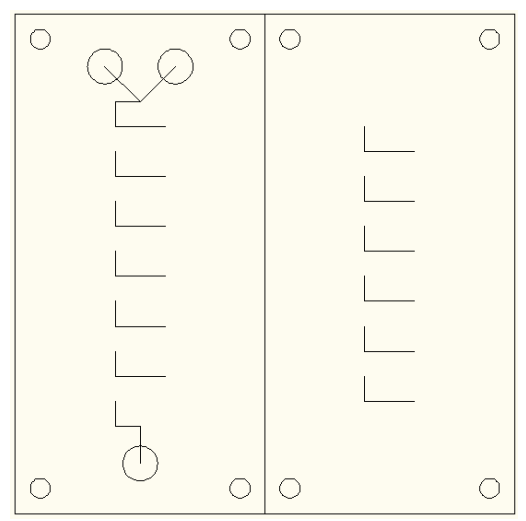

Figure 133 - AutoCAD design for passive 3-D serpentine mixing chip. This design would create two distinct chips with the left chip flipping along the dividing line onto the right chip and aligning with pins through the holes in the corners. 
Using a design consideration from polymer injection science, alignment holes are placed in the corners of the chips. The left chip'a pins are pressed into these alignment holes so the chips can be aligned based on the machine's fabrication and not based on visual accuracy of the fabricator. The two injection holes would be used to inject two different species that require mixing. The two species would then travel through the tortuous path down the chip, hopefully with successful outcomes similar to those of Liu et al.. ${ }^{77}$

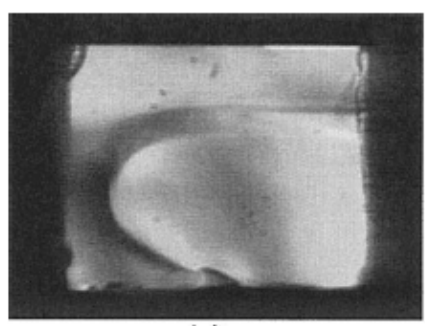

(a)

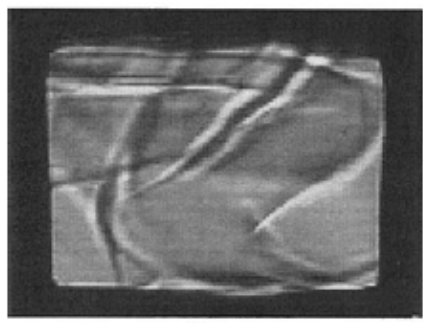

(b)

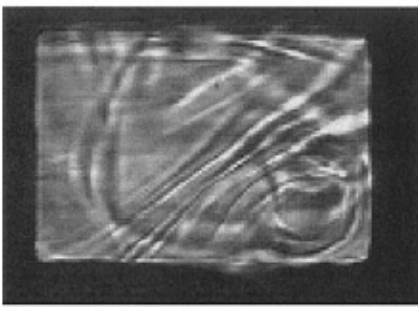

(c)

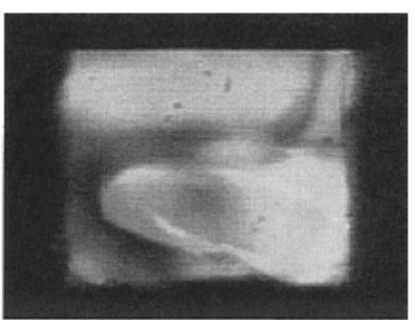

(d)

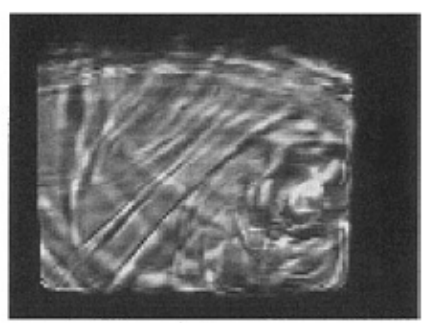

(e)

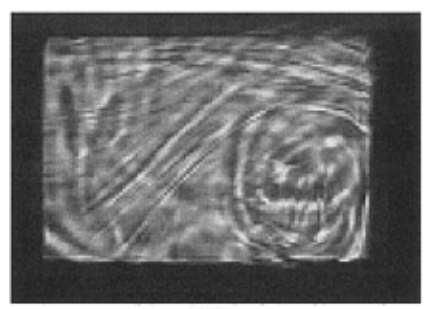

(f)

Figure 134 - Successively increasing levels of mixing of water and alcohol along a tortuous microfluidic channel ${ }^{77}$

Lastly, polymer microfluidics can be combined into a series of chips either by combining the CAD drawings into a single file and attaching the outlets of one chip into the inlets of 
the next or manufacturing the chips separately and attaching them together using one of the many bonding methods. Creating all the chips on one sheet is easier to scale than with PDMS devices as the researcher is not constrained by silicon wafer or aligner sizes for mold creation. A CAD file for separately-manufactured chips is shown below.
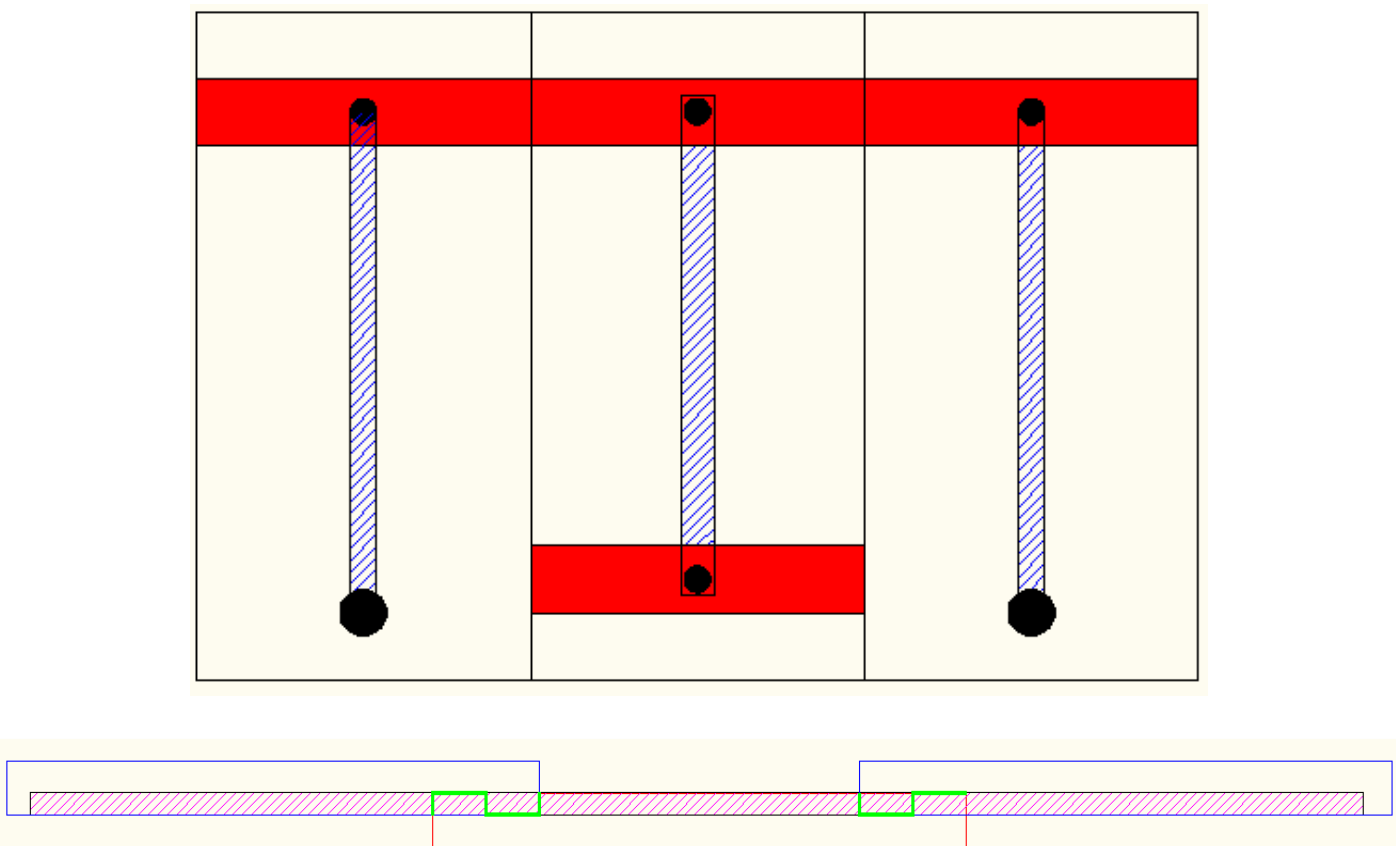

Figure 135 - Series mixing microfluidic device with rough channel floor to facilitate mixing (T) and the side profile of how the chips will be attached (B) In the top figure, the black lines are full laser power to separate the chips and create through-holes, the red lines are rastered cuts used to create pits in the material and the blue hatch is the rastered channel created for fluid flow. In the bottom figure, the green lines show interfaces between the chips and the hatched region shows the fluid channel

This setup could be used to create modular chip designs so custom setups could be created based on end-user needs. Sealing between the interfaces could be accomplished using the PMMA/PDMS/PMMA bonding method mentioned in the Alternative Manufacturing Methods section. 


\subsection{Final Thoughts}

PDMS devices are ubiquitous in the microfluidic world but hardly any have reached the stage of manufacturability required for field use. Microfluidics concepts have been proven, but now the science is at the crucial stage to prove its real-world usability with low-cost manufacturing methods. This thesis should be taken as guide on how to approach creating a low-cost microfluidic device. Many manufacturing methods were attempted, some not brought up in this thesis. For all of these manufacturing methods a pilot study was performed to assess feasibility and methods that did not show initial promise were abandoned. This does not mean that methods such as IPA/microwave bonding or sewn electrodes are worthless methods; it simply means these methods require additional investigation before they can be deemed useful. Many of the manufacturing steps were tried several different ways. PDMS microfluidics manufacturing is a very firmly-set science with strict rules, but to be truly innovative and productive a researcher must not be afraid to strike out on their own and attempt methods never tried before. PMMA microfluidics, and especially laser-etched PMMA, is still in its infancy and may only have a fraction of the capability of more established microfluidics platforms but it shows great promise and is worthy of additional research. 


\section{Works Cited}

1. Curtis D. Chin, Vincent Linder and Samuel K. Sia, Lab-on-a-chip devices for global health: Past studies and future opportunities. Lab on a Chip, 2007. 7: p.41-57.

2. Abbot Point of Care Technologies. [Biomedical Devices] $2008<$ http://www.abbottpointofcare.com/>

3. Ken-ichi Ohno, Kaoru Tachikawa, Andreas Manz. Microfluidics: Applications for analytical purposes in chemistry and biochemistry. Electrophoresis 2008. 29: p. 4443-4453.

4. Blain Christen J, Andreou AG. Design, Fabrication, and Testing of a Hybrid CMOS/PDMS Microsystem for Cell Culture and Incubation. IEEE Transactions on Biomedical Circuits and Systems, 2007. 1(1):3-18.

5. Duffy DC, McDonald JC, Schueller OJ, Whitesides GM. Rapid Prototyping of Microfluidic Systems in Poly(dimethylsiloxane). Analytical Chemistry, 1998. 70(23): p. 4974-4984.

6. McDonald JC, Whitesides GM. Poly(dimethylsiloxane) as a material for fabricating microfluidic devices. Accounts of Chemical Research, 2002. 35(7): p. 491-9.

7. Fujii T, PDMS-based microfluidic devices for biomedical applications. Microelectronic Engineering. 2002. 62: p. 907-914.

8. Samuel K. Sia, George M. Whitesides, Microfluidic devices fabricated in poly(dimethylsiloxane) for biological studies. Electrophoresis 2003. 24: p. 35633576.

9. Brown L, Koerner T, Horton JH, Oleschuk RD. Fabrication and characterization of poly(methylmethacrylate) microfluidic devices bonded using surface modifications and solvents. Lab on a chip, 2006. 6(1): p. 66-73.

10. Nikhil S. Tambe, Bharat Bhushan. Micro/nanotribological characterization of PDMS and PMMA used for BioMEMS/NEMS applications. Ultramicroscopy, 2005. 105: p. $238-247$

11. Stephen Y. Chou,, Peter R. Krauss, Wei Zhang, Lingjie Guo, and Lei Zhuang. Sub$10 \mathrm{~nm}$ imprint lithography and applications. Journal of Vacuum Science Technology, 1997. 15(6) 2897-2904 
12. Haiducu M, Rahbar M, Foulds IG, et al..Deep-UV patterning of commercial grade PMMA for low-cost, large-scale microfluidics. Journal of Micromechanics and Microengineering, 2008. 18(11): p. 115-129.

13. Paul Yager, Thayne Edwards, Elain Fu, Kristen Helton, Kjell Nelson, Milton R. Tam, Bernhard H. Weigl. Microfluidic diagnostic technologies for global public health. Nature, 2006, 442: p. 412-418.

14. Peter Gascoyne, Jutamaad Satayavivad, Mathuros Ruchirawat. Microfluidic approaches to malaria detection. Acta Tropica, 2004. 89: p. 357-369.

15. Pineda MF, Chan LL, Kuhlenschmidt T, et al.. Rapid Specific and Label-Free Detection of Porcine Rotavirus Using Photonic Crystal Biosensors. IEEE Sensors Journal, 2009. 9(4): p. 470-477.

16. D. Stevenson, I.D.W., Sample Preparation for Biomedical and Environmental Analysis. 1994, New York: Plenum Press.

17. Xiangqun Xu,a, Yeh-Chan Ahn,b and Zhongping Chenb. Feasibility of Doppler variance imaging for red blood cell aggregation characterization. Journal of Biomedical Optics, 2009. 14(6). 060507-1-060507-3

18. H. Zhanga,b, S.D. Zhaib, Y.M. Lia, L.R. Chen. Effect of different sample pretreatment methods on the concentrations of excitatory amino acids in cerebrospinal fluid determined by high-performance liquid chromatography. Journal of Chromatography, 2003. 784: p. 131-135.

19. Manz A. Microfluidics : Applications for analytical purposes in chemistry and biochemistry. Electrophoresis, 2008. 29(22): p. 4443-4453.

20. Liu J, Pan T, Woolley AT, Lee ML. Surface-modified poly(methyl methacrylate) capillary electrophoresis microchips for protein and peptide analysis. Analytical chemistry, 2004. 76(23): p. 6948-6955.

21. Nelson DL and Cox MM., Lehninger Principles of Biochemistry (4th ed). 2005, New York: Freeman

22. Patrick Camilleri, Capillary Electrophoresis Theory and Practice. 2nd Edition. 1998. CRC Press Florida 
23. Vrbová Barbora. Analysis of Images from Gel Electrophoresis. Czech Technical University in Prague, Department of Cybernetics. May 2008

24. Adam T. Woolley and Richard A. Mathies. Ultra-High-speed DNA Sequencing Using Capillary Electrophoresis Chips. Analytical Chemistry, 1995. 67: p. 3676-3680.

25. Dolník V., Liu S., Jovanovich, S. Capillary electrophoresis on microchip CE and CEC. Electrophoresis, 2000. 21: p. 41-54.

26. Woolley T, Lao K, Glazer N, Mathies R. Capillary electrophoresis chips with integrated electrochemical detection. Analytical Chemistry, 1998. 70(4): p. 684-688.

27. Probstein, Ronald F, Physicochemical Hydrodynamics. 2nd Edition. John Wiley \& Sons 1994.

28. Butt, Has-Jurgen; Graf, Karlheinz; Kappl, Michael, Physics and Chemistry of Interfaces. Wiley-VCH Verlag GmbH \& Co. KGaA, Weinheim 2003

29. Kirby BJ, Hasselbrink EF. Zeta potential of microfluidic substrates:Data for polymers. Electrophoresis, 2004. 25(2): p. 203-213.

30. K. Nortemann, J. Hilland, and U. Kaatze. Dielectric Properties of Aqueous $\mathrm{NaCl}$ Solutions at Microwave Frequencies. Journal of Physical Chemistry A, 1997. 101: p. 6864-6869.

31. Klank H, Kutter JP, Geschke O. $\mathrm{CO}_{2}$-laser micromachining and back-end processing for rapid production of PMMA-based microfluidic systems. Lab on a chip, 2002. 2(4): p. 242-246.

32. J Powell, G Ellis, IA Menzies, CO2 laser cutting of non-metallic materials: Lasers in Manufacturing, Springer, 1987.

33. Detlef Snakenborg, Henning Klank and Jorg P Kutter. Microstructure fabrication with a CO2 laser system. Journal of Micromechanics and Microengineering, 2004. 14: p. 182-189.

34. Lin Y, Chang H. Modification of poly(methyl methacrylate) microchannels for highly efficient and reproducible electrophoretic separations of double-stranded DNA. Journal of Chromatography, 2005. 1073(1-2): p. 191-199. 
35. Fu L, Lin C. High-resolution DNA separation in microcapillary electrophoresis chips utilizing double-L injection techniques. Electrophoresis, 2004. 25(21-22): p. $3652-$ 3659.

36. Jacobson SC, Koutny LB, Hergenroeder R, Moore AW, Ramsey JM. Microchip Capillary Electrophoresis with an Integrated Postcolumn Reactor. Analytical Chemistry, 1994. 66(20): p. 3472-3476.

37. Rahbar M, Chhina S, Sameoto D, Parameswaran M. Microwave-induced, thermally assisted solvent bonding for low-cost PMMA microfluidic devices. Journal of Micromechanics and Microengineering, 2010. 20(1): p. 15-26.

38. Lin C, Yang H, Chao C. Hexagonal microlens array modeling and fabrication using a thermal reflow process. Journal of Micromechanics and Microengineering, 2003. 13(5): p. 775-781.

39. Bruin G.J.M. Recent developments in electrokinetically driven analysis on microfabricated devices. Electrophoresis, 2000. 21: p. 3931-3951.

40. Rajiv Bharadwaj Juan G. Santiago Bijan Mohammadi. Design and optimization of on-chip capillary electrophoresis. Electrophoresis, 2002. 23: p. 2729-2744

41. Intentionally left blank

42. Paegel BM, Hutt LD, Simpson PC, Mathies R. Turn geometry for minimizing band broadening in microfabricated capillary electrophoresis channels. Analytical chemistry, 2000. 72(14): p. 3030-7.

43. Horng R, Han P, Chen H, et al.. PMMA-based capillary electrophoresis electrochemical detection microchip fabrication. Journal of Micromechanics and Microengineering, 2005. 6: p. 1-6.

44. Joseph Wang, Martin Pumera, Madhu Prakash Chatrathi, Alberto Escarpa, Renate Konrad, Anja Griebel, Wolfgang Dörner, Holger Löwe. Towards disposable lab-ona-chip: Poly(methylmethacrylate) microchip electrophoresis device with electrochemical detection. Electrophoresis, 2002. 23: p. 596-601

45. Ashby, Michael F., Materials Selection in Mechanical Design (3rd Edition). 2005 Elsevier Massachusetts. 
46. Lin Y, Chang H. Modification of poly(methyl methacrylate) microchannels for highly efficient and reproducible electrophoretic separations of double-stranded DNA. Journal of Chromatography A, 2005. 1073(1-2): p. 191-199.

47. Zhu X, Liu G, Guo Y, Tian Y. Study of PMMA thermal bonding. Microsystem Technologies, 2006. 13(3-4): p. 403-407.

48. Siber, Anthony. "Konstrukcija stvarnosti" <http://asiber.ifs.hr/>

49. KLA Tencor. [Inspection and Metrology] $2010<$ http://www.kla-tencor.com/>

50. Chow WW, Lei KF, Shi G, Li WJ, Huang Q. Microfluidic channel fabrication by PDMS-interface bonding. Smart Materials and Structures, 2006. 15(1): p. S112-S116.

51. Shiu PP, Knopf GK, Ostojic M, Nikumb S. Rapid fabrication of tooling for microfluidic devices via laser micromachining and hot embossing. Journal of Micromechanics and Microengineering, 2008. 18(2):025012.

52. McMaster Carr. [Industrial Supply] <http://www.mcmaster.com/>

53. Cheng J. Direct-write laser micromachining and universal surface modification of PMMA for device development. Sensors and Actuators B: Chemical, 2004. 99(1): $\mathrm{p}$. 186-196.

54. Savage, R. "Materials Analysis - Scanning Probe Microscopy." Materials Engineering 510. Cal Poly State University, San Luis Obispo, California. Spring 2010.

55. Pham D, Tonge L, Cao J, et al.. Effects of polymer properties on laser ablation behaviour. Smart Materials and Structures, 2002. 11(5): p. 668-674.

56. Jennifer Monahan, Andrew A. Gewirth, and Ralph G. Nuzzo. A Method for Filling Complex Polymeric Microfluidic Devices and Arrays. Analytical Chemistry, 2001.

73: p. 3193-3197

57. Janini GM, Metral CJ, Issaq HJ, Muschik GM. Peptide mobility and peptide mapping in capillary zone electrophoresis. Experimental determination and theoretical simulation. Journal of Chromatography A, 1999. 848(1-2): p. 417-33. 
58. Luo LW, Teo CY, Ong WL, et al.. Rapid prototyping of microfluidic systems using a laser-patterned tape. Journal of Micromechanics and Microengineering, 2007. 17(12): p. N107-N111.

59. Philippe Lam, Kenneth J. Wynne, and Gary E. Wnek. Surface-Tension-Confined Microfluidics. Langmuir, 2002. 18: p. 948-951

60. Ng JM, Gitlin I, Stroock AD, Whitesides GM. Components for integrated poly(dimethylsiloxane) microfluidic systems. Electrophoresis, 2002. 23(20): p. 34613473

61. Becker H. Hot embossing as a method for the fabrication of polymer high aspect ratio structures. Sensors and Actuators A. Physical, 2000. 83(1-3): p. 130-135

62. Lee G. Microfabricated plastic chips by hot embossing methods and their applications for DNA separation and detection. Sensors and Actuators B: Chemical, 2001. 75(1-2): p.142-148

63. Chen Y.-H., Chen S.-H. Analysis of DNA fragments by microchip electrophoresis fabricated on poly(methylmethacrylate) substrates using a wire-imprinting method. Electrophoresis, 2000. 21: p. 165-170

64. Larisa Martynova, Laurie E. Locascio, Michael Gaitan, Gary W. Kramer, Richard G. Christensen, and William A. MacCrehan. Fabrication of Plastic Microfluid Channels by Imprinting Methods. Analytical Chemistry, 1997. 69: p. 4783-4789

65. Becker H., Gartner C. Polymer microfabrication methods for microfluidic analytical applications. Electrophoresis, 2000. 21: p. 12-26

66. Lee L., Madou M., Koelling K., Daunert S., Lai S., Koh C., Juang Y., Lu Y, Yu. L. Design and Fabrication of CD-Like Microfluidic Platforms for Diagnostics. Polymer-Based Microfabrication. Biomedical Microdevices, 2001. 3(4): p. 339-351

67. Becker H, Locascio LE. Polymer microfluidic devices. Talanta, 2002. 56(2): p. 26787

68. Holger Becker, Ulf Heim. Hot embossing as a method for the fabrication of polymer high aspect ratio structures. Sensors and Actuators, 2000. 83: p. 130-135 
69. V. Studer, A. Pe' pin, and Y. Chen, Nanoembossing of thermoplastic polymers for microfluidic applications. Applied Physics Letters. 80(19) p. 3414-3416.

70. Dong-Mung Tsai, Kuan-Wen Lin, Jyh-Myng Zen, Hung-Yu Chen, Ray-Hua Hong, A new fabrication process for a microchip electrophoresis device integrated with a three-electrode electrochemical detector. Electrophoresis, 2005. 26: p. 3007-3012

71. Datta P, George G, Tiwari S, Goettert J. Monolithic fabrication of electro-fluidic polymer microchips. Microsystem Technologies, 2008. 15(3): p. 463-469.

72. Cha J., DNA hybridization electrochemical sensor using conducting polymer. Biosensors and Bioelectronics, 2003. 18(10): p. 1241-1247

73. Andrew G. Hadd, Daniel E. Raymond, John W. Halliwell, Stephen C. Jacobson, and J. Michael Ramsey, Microchip Device for Performing Enzyme Assays. Analytical Chemistry, 1997. 69: p. 3407-3412

74. F.A. Gomez, Biological Applications of Microfluidics. John Wiley \& Sons, New Jersey 2008.

75. Becker H., Gartner C. Polymer microfabrication methods for microfluidic analytical applications. Electrophoresis, 2000. 21: p. 12-26

76. Pescetti D. Micellar Electrokinetic Chromatography. California Polytechnic State University, San Luis Obispo, CA.

77. Liu R, Stremler M, Sharp K, et al.. Passive mixing in a three-dimensional serpentine microchannel. Journal of Microelectromechanical Systems, 2000. 9(2): p. 190-197. 